

\section{DISCLAIMER}

This report was prepared as an account of work sponsored by an agency of the United States Government. Neither the United States Government nor any agency Thereof, nor any of their employees, makes any warranty, express or implied, or assumes any legal liability or responsibility for the accuracy, completeness, or usefulness of any information, apparatus, product, or process disclosed, or represents that its use would not infringe privately owned rights. Reference herein to any specific commercial product, process, or service by trade name, trademark, manufacturer, or otherwise does not necessarily constitute or imply its endorsement, recommendation, or favoring by the United States Government or any agency thereof. The views and opinions of authors expressed herein do not necessarily state or reflect those of the United States Government or any agency thereof. 


\section{DISCLAIMER}

Portions of this document may be illegible in electronic image products. Images are produced from the best available original document. 


$$
\begin{array}{r}
\text { ORNL/Sub/86-SA094/2 } \\
\text { ORNL/Sub/86-SA224/2 } \\
\text { HR/JAI-289 } \\
\text { Dist. Categories UC-71, }-85
\end{array}
$$

\title{
USE OF TRANSPORTABLE STORAGE CASRS IN THE NUCLEAR WASTE MANAGEMENT SYSTEM
}

\author{
Appendices
}

December 1987
Program Sponsored by the Office of Civilian Radioactive Waste Management Systems Integration Program

ORNL/SUb--86-SA094/2

DE88 007846

\section{Prepared by}

H\&R Technical Associates, Ine. 545 Oak Ridge Turnpike

Oak Ridge, Tennessee 37831
E. R. Johnson Associates, Inc. 10461 White Granite Drive, Suite 204 Oakton, Virginia 22124

Under Subcontract Numbers $41 \mathrm{X}-\mathrm{SA} 094 \mathrm{~V}$ and $30 \mathrm{X}-\mathrm{SA} 224 \mathrm{~V}$

for

Oak Ridge National Laboratory

Oak Ridge, Tennessee 37831

operated by

MARTIN MARIETTA ENERGY SYSTEMS, INC.

for the

U.S. DEPARTMENT OF ENERGY

under contract DE-AC05-84OR21400 


\section{LIST OF APPENDICES}

APPENDIX A ESTIMATED CAPITAL COSTS FOR METAL AND CONCRETE STORAGE CASKS

APPENDIX B ESTIMATED COST OF HANDLING OPERATIONS AT THE REACTOR SITE INVOLVING SPENT FUEL DRY STORAGE MODULES, TRANSPORT CASKS, AND SOC OVERPACKS

APPENDIX C ESTIMATES OF LIFE CYCLE AND UNIT COSTS FOR USE OF DRY SPENT FUEL STORAGE MODULES, TRANSPORT CASKS, AND SOC OVERPACKS AT REACTOR SITES

APPENDIX D ESTIMATED COSTS FOR TRANSPORT OF SPENT FUEL FROM REACTOR SITES TO DOE FACILITIES IN STANDARD TRANSPORT CASKS, TSCS, AND SOCS (BOTH WITH AND WITHOUT OVERPACKS)

APPENDIX E COST OF LAG STORAGE

APPENDIX $F$ ESTIMATED COST FOR MRS MODULES

APPENDIX G ESTIMATED COSTS FOR TRANSPORT OF SPENT FUEL FROM MRS TO REPOSITORY FACILITIES IN TSCS AND STANDARD TRANSPORT CASKS

APPENDIX H ESTIMATED COSTS FOR SALVAGE OR DISPOSAL OF USED TSCS, SOCS AND CONCRETE STORAGE CASKS

APPENDIX I SENSITIVITY OF COSTS TO VARIATIONS IN THE CAPACITY AND FABRICATION COSTS OF METAL STORAGE CASKS

APPENDIX J PROSPECTS FOR USE OF TSCS IN CONNECTION WITH THE STORAGE AND HANDLING OF DEFENSE WASTES 
APPENDIX A

ESTIMATED CAPITAL COSTS FOR METAL AND CONCRETE STORAGE CASKS 
APPENDIX A

ESTIMATED CAPITAL COSTS FOR METAL AND CONCRETE STORAGE CASKS

TABLE OF CONTENTS

1.0 METAL CASKS (Storage Only) A-1

2.0 TRANSPORTABLE STORAGE CASKS A-4

3.0 CONCRETE STORAGE CASKS A-7

4.0 REFERENCES FOR APPENDIX A A-10 
The purpose of this Appendix is to develop an estimate of the capital cost of metal and concrete storage casks so that the capital costs associated with the use of a TSC for at-reactor storage can be compared with those of using a SOC and a concrete cask.

\subsection{METAL CASKS (STORAGE ONLY)}

This section discusses the capital costs associated with metal casks that are designed and licensed for at-reactor storage of spent fuel. It was assumed that at the time of their design and licensing, no attempt would be made to certify them for use as a transport casks even though they may be able to be used for a one-time shipment or placed in a protective package and shipped at the end of the storage period.

Discussions were held with two separate designers of metal storage casks to obtain the current estimated costs for such casks. One firm estimated that a metal cask designed for storage-oniy of 24 PWR or 52 BWR assemblies would cost about $\$ 1.05-m i l l i o n$ (1987 dollars). A second cask designer estimated the cost for a 21 PWR assembly cask at $\$ 1.0$-million (1987 dollars); this cask could accommodate as many as 57 BWR assembiies. Included in these costs was the cost of performing the initial design of the cask and the project management activities associated with the cask design and fabrication effort (licensing, quality assurance, etc.). These costs are summarized in Table $\mathrm{A}-1$.

TABLE A-1

ESTIMATED COST OF DESIGN AND LICENSING OF SOCS

$(\$ 000,1987)$

Description

Design and Engineering

Project Management \& Licensing Total
First

Two Casks

$\$ 534$

600

$\$ 1,134$
Each Additional

Cask

$\$ 32$

$\$ 32$ 
For the purposes of this study, it was assumed that a storage cask having a capacity for storing 21 PWR assemblies or 46 BWR assemblies would cost \$1.0-million (1987 dollars). This was believed to represent a conservative composite of the cost estimates obtained from cask design firms. Moreover, it was assumed that this cost assumed that the design and initial production costs were to be written off over the first 8 casks produced. This would put the cost of a cask, before design and initial production costs were added, at about $\$ 830$-thousand. Assuming that the cost of procurement management, fabrication follow and the like would amount to 10 percent of the cost of fabrication, the actual cost of the fabrication would then amount to about $\$ 755$-thousand. This compared favorably with an independent estimate of the cost of cask manufacture developed during the course of the study.

The prospective reduction of fabrication costs as more casks are produced was considered. The nodular iron cask costs described by Sorenson in Reference (1) and the Transnuclear cost data shown in Reference (2) were subjected to several curve fitting analyses. As a result, the following algorithm was developed which shows the relationship of cask fabrication cost to the number of casks that have been produced:

$$
\begin{aligned}
& c_{n}=c_{i} n^{-.046} \\
& \text { where: } \quad c_{n}=\text { cost of the nth cask produced } \\
& c_{i}=\text { cost of the initial cask produced }
\end{aligned}
$$

By using the foregoing relationship, and writing off the design and development costs over the first 10 casks produced, the cost of SOCs were calculated for various stages in the "learning curve", as shown in Table A-2. 
TABLE $A-2$

ESTIMATED COSTS FOR SOCS

(1987 Dollars)

\begin{tabular}{|c|c|c|c|c|c|c|c|c|}
\hline & \multicolumn{4}{|c|}{ Average Cost of SOC $(\$ 000)$} & \multicolumn{2}{|c|}{$\begin{array}{l}\text { SOC Capacity } \\
\text { (MTU) }\end{array}$} & \multicolumn{2}{|c|}{$\begin{array}{c}\text { Cost/Unit Capacity } \\
(\$ / \mathrm{kgU})\end{array}$} \\
\hline & $\begin{array}{l}\text { Design \& } \\
\text { Licensing }\end{array}$ & $\begin{array}{l}\text { Fabric- } \\
\text { ation }\end{array}$ & $\begin{array}{l}\text { Admin \& } \\
\text { Procure- } \\
\text { ment }\end{array}$ & Total & $\begin{array}{l}\text { Intact } \\
\text { Fuel }\end{array}$ & $\begin{array}{l}\text { Con- } \\
\text { solidated } \\
\text { Fuel }\end{array}$ & $\begin{array}{l}\text { Intact } \\
\text { Fuel }\end{array}$ & $\begin{array}{l}\text { Con- } \\
\text { solidated } \\
\text { Fuel } \\
\end{array}$ \\
\hline First 10 Units $^{c}$ & 139 & 747 & 75 & 961 & 9.26 & 15.37 & 103.8 & 62.5 \\
\hline 50th Unit & - & 668 & 67 & 735 & 9.26 & 15.37 & 79.4 & 47.8 \\
\hline 100th Unit & - & 647 & 65 & 712 & 9.26 & 15.37 & 76.9 & 46.3 \\
\hline 500th Unit & - & 601 & 60 & 661 & 9.26 & 15.37 & 71.4 & 43.0 \\
\hline
\end{tabular}

a weighted average based on a cask capacity of 21 PWR or 46 BWR assemblies, a $2 / 1$ weight ratio of PWR to BWR fuel, and $461 \mathrm{kgJ} / P W R$ assembly and $183 \mathrm{kgU} / \mathrm{BWR}$ assembly

b Assumes fuel rods are consolidated and structural parts are compacted so together there is a net compaction ratio of 1.66

C Assumes fabrication cost for first cask produced is $\$ 800$-thousand. 
This section discusses the capital costs associated with metal casks that are designed and Ticensed for at-reactor storage of spent fuel and are certified for use as transport casks at the time they are manufactured.

Discussions were held with three separate designers of casks. One firm estimated that an additional $\$ 300$-thousand would be required to make its SOC certifiable for transport, because a more rugged design would be required for the baskets involved. A second designer believed that the basket used in connection with its cask was sufficiently rugged for use in transport service, and that no additional cost would be required to make its SOC certifiable for transport. A third designer believed that a TSC would cost about \$100thousand more than a SOC. Therefore, for the purposes of this study it was assumed that the fabrication cost (net of design, licensing, administration and procurement costs) for the first TSC produced would be \$100-thousand higher than a corresponding SOC (as described in Section 1.0 of this Appendix A), or $\$ 900$-thousand, to cover the added cost of manufacturing a cask of somewhat more rugged design than a SOC. It was assumed that the same algorithm that was developed for fabrication of SOCs in Section 1.0 of this Appendix A would be applicable for the fabrication of TSCS. However, in this case, in addition to the cost fabrication, it was assumed that 10 percent would have to be added for administration and procurement activities increasing the costs therefor to $\$ 990$-thousand. Moreover, it was also assumed that design and certification of the TSC would have to be added for the foregoing cost.

Both EG\&G and one cask designer estimated the cost of engineering and certification of a TSC at about \$3.0-million (1986 dollars); this would amount to about \$3.2-million in 1987 dollars. In a study performed for DOE in 1983-1985, Transnuclear, Inc. (TNI) estimated the cost of design and certification of a transport cask at about $\$ 3.5$-million in 1985 dollars (Reference (1)). As a result of a review of the latter costs, it was concluded that they would also be applicable to the design and certification of a TSC. The personnel-related costs associated with the foregoing TNI estimate were escalated by 6.6 percent, and the cost of machinery and equipment included in the estimate was escalated by 5 percent, to convert the costs to 1987 dollars. These cost estimates for the design and certification of TSCS, are summarized in the following Table A-3. 
TABLE A-3

ESTIMATED COST OF DESIGN \& CERTIFICATION OF TSCS

$(\$ 000,1987)$

\section{Description}

Design \& Engineering

Testing \& Certification

Procurement Activities

Associated With Prototype

Fabrication Follow

Associated with Prototype

Project Management \& Licensing

Total
First

Two Casks

$\$ 858$

1,192

103

91

$\frac{1,450}{\$ 3,694}$
Each Additional

Cask

$\$-$

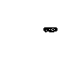

32

$\$ 32$

Applying the algorithm that was developed in Section 1.0 of this Appendix $A$ to the cost of fabrication of a TSC, 10 percent of the cost of fabrication for procurement management and fabrication follow, and writing off the design and certification costs over the first 10 casks produced, the costs of TSCS were calculated for various stages of the "learning curve", as shown in Table A-4. 
TABLE A-4

ESTIMATED COSTS FOR TSCS

(1987 Dollars)

\begin{tabular}{|c|c|c|c|c|c|c|c|c|}
\hline & \multicolumn{4}{|c|}{ Cost of TSC $(\$ 000)$} & \multicolumn{2}{|c|}{$\begin{array}{l}\text { TSC Capacity } \\
\text { (MTU) }\end{array}$} & \multicolumn{2}{|c|}{$\begin{array}{c}\text { Cost/Unit Capacity } \\
(\$ / \mathrm{kgU}) \\
\end{array}$} \\
\hline & $\begin{array}{l}\text { Design \& } \\
\text { Licensing } \\
\end{array}$ & $\begin{array}{l}\text { Fabric- } \\
\text { ation }\end{array}$ & $\begin{array}{l}\text { Admin \& } \\
\text { Procure- } \\
\text { ment }\end{array}$ & Total & $\begin{array}{l}\text { Intact } \\
\text { Fuel }\end{array}$ & $\begin{array}{l}\text { Con- } \\
\text { solidated } \\
\text { Fuel }\end{array}$ & $\begin{array}{l}\text { Intact } \\
\text { Fuel }\end{array}$ & $\begin{array}{l}\text { Con- } \\
\text { solidated } \\
\text { Fuel }\end{array}$ \\
\hline First 10 Units $^{c}$ & $\$ 395$ & $\$ 840$ & $\$ 84$ & $\$ 1,319$ & 9.26 & 15.37 & $\$ 142.4$ & $\$ 85.8$ \\
\hline 50 th Unit & - & 752 & 75 & 827 & 9.26 & 15.37 & 89.3 & 53.8 \\
\hline 100th Unit & - & 728 & 73 & 801 & 9.26 & 15.37 & 86.5 & 52.1 \\
\hline 500 th Unit & - & 676 & 68 & 744 & 9.26 & 15.37 & 80.3 & 48.4 \\
\hline
\end{tabular}

\footnotetext{
a Weighted average based on a cask capacity of 21 PWR or 46 BWR assemblies, a $2 / 1$ weight ratio of PWR to BWR fuel, and $461 \mathrm{kgU} / \mathrm{PWR}$ assembly and 183/BWR assembly

b Assumes fuel rods are consolidated and structural parts are compacted so together there is a net compaction ratio of 1.66

${ }^{c}$ Assumes fabrication cost for first cask produced is $\$ 900$-thousand.
} 
This section discusses the capital costs associated with concrete storage casks, designed and licensed for at-reactor storage. While it was not the basic purpose of this study to perform a comparative assessment of the use of metal storage casks and concrete storage modules, it was necessary to make an assessment of any increased costs a utitity might incur in using TSCS over other dry storage alternatives that could be used at reactor sites in order to properly determine the extent to which the use of a TSC was viable. The viability of use of TSCS relies on the cost (and other) advantages to the combined utility/DOE spent fuel management system at least offsetting the disadvantages involved.

Discussions were held with Nuclear Packaging Inc. (NUPAC) and NUTECH, Inc. regarding estimated costs of :torage of spent fuet in concrete storage modules.

As a result of these discussions it was assumed that the concrete storage cask would have a capacity for storing 9 PWR assemblies or 25 BWR assemblies, for an average capacity of 4.291 MTU/cask. The cost of the first cask buitt was estimated at $\$ 150$-thousand and the design and development cost of the cask and its loading and unloading equipment was estimated to be $\$ 1.75$ million.

A learning curve similar to that described in Section 1.0 of this Appendix $A$ for metal storage casks was applied to the cost of manufacture of concrete casks, even though such may not be as dramatic inasmuch as concrete casks will probably be produced near the location they are used. This means there are likely to be many more locations producing concrete casks than metal casks, with limited numbers being produced at any given location. However, the same learning curve effect was used for concrete casks as for metal casks since it was assumed that the designer would procure the concrete cask fabrication services and would pass along his fabrication knowledge to the fabricator.

The cost of concrete storage casks were calculated for various stages in the learning curve as shown in Table A-5. 
TABLE A-5

ESTIMATED COSTS FOR CONCRETE STORAGE CASKS

(1987 Dollars)

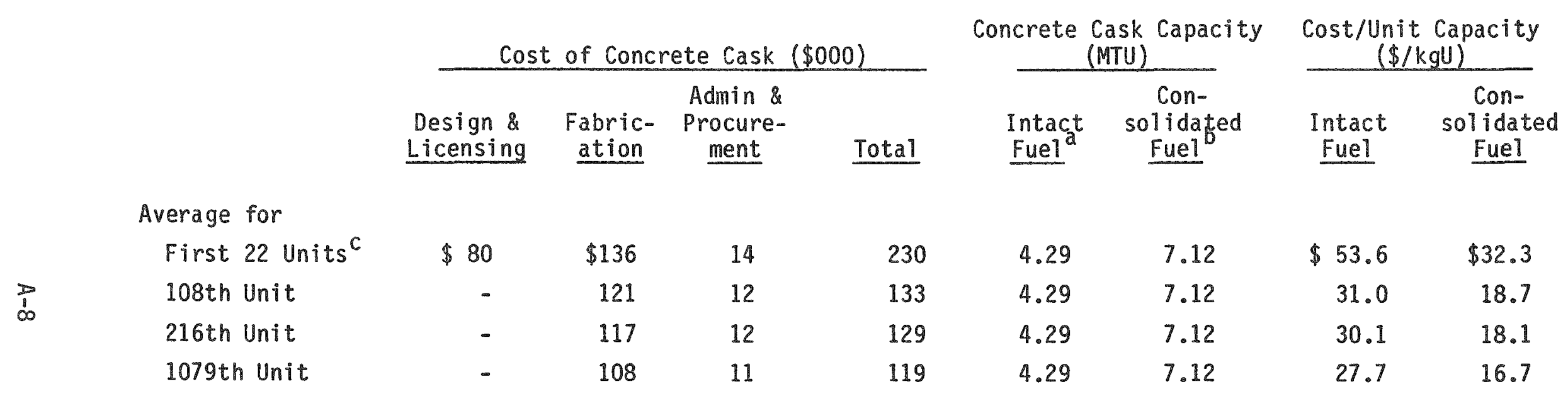

\footnotetext{
a Weighted average based on a cask capacity of 9 PWR and 25 BWR assemblies, a $2 / 1$ weight ratio of PWR to BWR fuel, and $461 \mathrm{kgU} / P W R$ assembly and $183 \mathrm{kgU} /$ BWR assembly

$\mathrm{b}$ Assumes fuel rods are consolidated and structural parts are compacted so together there is a net compaction ratio of 1.66

C Assumes fabrication cost for first cask produced is $\$ 150$-thousand.
} 
In addition to the concrete casks, it is necessary for a utility to have a means of transferring the spent fuel from the reactor pool to the cask loading area, and for loading the casks. This requires a transfer cask, a plug cask, a loading collar, and a platform on which the operations are conducted. This type of equipment is currently in use at Three Mile Island for loading of a transport cask with canisters of fuel material that has been recovered from the reactor. In this case the pool water is too heavily contaminated to load the transport cask in the pool. In the case of concrete casks it has been assumed that these will have to be loaded outside the pool. The cost of the equipment was estimated at about \$1.0-million (1987 dollars). It was assumed that $\$ 750$-thousand of this equipment could be leased from a service company, but that $\$ 250$-thousand would represent a needed capital investment by the utility.

The cost of leasing the loading equipment was estimated to be $\$ 1.45 / \mathrm{kgU}$ for intact fuel assemblies and $\$ 1.26 / \mathrm{kgU}$ for consolidated fuel. This is based on the following assumptions:

(1) Annual revenue requirements of the lessor for use of the equipment would be $\$ 226.4$-thousand, assuming:

(a) a 15 percent discounted cash flow return on investment after taxes

(b) a 10-year depreciation period

(c) a 45 percent Federal tax rate

(d) a 6 percent state tax rate

(e) property taxes and insurance at $\$ 1 / \$ 100$ valuation

(2) The equipment would be used at 6 different reactor sites during the year for intact fuel assemblies or 7 different sites during the year for consolidated fuel: 28 MTU of spent fuel would be loaded at each site

(3) It would cost $\$ 3,000$ to move the equipment between sites

(4) Set up and takedown time would amount to 7 days each, shipping time would amount to 7 days, 6.7 days would be required for loading an annual discharge of intact fuel assemblies, 4.5 days would be required for loading an annual discharge of consolidated fuel, and a 50\% utilization efficiency would be realized for the use of the casks. 
In addition to the costs shown in Appendix $B$ for operations at the reactor, there is a total capital cost of $\$ 395$-thousand required in connection with the use of the concrete cask at a reactor site, \$145-thousand of which is for canning equipment and $\$ 250$-thousand which is for loading equipment.

It should be pointed out that there has been little demonstration work performed to date on the viability of the use of concrete storage modules for at-reactor storage of spent fuel. While there is no apparent reason why such storage would not prove feasible from an operating standpoint, the costs associated with such storage are less certain than those involved in the use of metal storage casks.

\subsection{REFERENCES FOR APPENDIX A}

(1) K. B. Sorenson, Cask Materials Cost Comparison Report, Sandia National Laboratories, October 17, 1986

(2) E. R. Johnson Associates, Inc., Assessment of the Use of a Multi-Purpose and Centralized Facility for the Disassembly and Packaging of Spent Nuclear Fuel to Support the Various Segments of the DOE Waste Management System, JAI-254, DOE Contract NO. DE-ACO1-84RW00037 
APPENDIX B

ESTIMATED COST OF HANDLING OPERATIONS AT THE REACTOR SITE INVOLVING SPENT FUEL DRY STORAGE MODULES, TRANSPORT CASKS, AND SOC OVERPACKS 
APPENDIX B

ESTIMATED COST OF HANDLING OPERATIONS AT THE REACTOR SITE INVOLVING

SPENT FUEL DRY STORAGE MODULES, TRANSPORT CASKS, AND SOC OVERPACKS

TABLE OF CONTENTS

Page

1.0 ESTIMATED COST OF RECEIPT OF DOE-FURNISHED TRANSPORT

CASKS OR METAL STORAGE CASK AND PREPARATION FOR LOADING

2.0 ESTIMATED COST OF LOADING A TSC OR SOC AND PLACEMENT B-6

IN STORAGE

3.0 ESTIMATED COST OF LOADING A CONCRETE CASK AND PLACEMENT B-8

IN STORAGE

3.1 Estimated Cost of Canning B-8

3.2 Estimated Cost of Loading Concrete Casks B-10

3.3 Estimated Cost of Receipt of Concrete Cask, Closure B-10 After Loading, and Placement in Storage

4.0 ESTIMATED COST OF REMOVAL OF A SOC FROM STORAGE AND $B-12$

UNLOADING IN REACTOR POOL

5.0 ESTIMATED COST OF REMOVAL OF A TSC FROM STORAGE AND B-12 PREPARATION FOR SHIPMENT

6.0 ESTIMATED COST OF REMOVAL OF A CONCRETE CASK FROM STORAGE B-15 AND UNLOADING

$\begin{array}{ll}\text { 7.0 ESTIMATED COST OF INSPECTION } & \text { B-17 }\end{array}$

8.0 ESTIMATED COST OF LOADING A TRANSPORT CASK AND PREPARING B-18

IT FOR SHIPMENT TO A DOE SITE

9.0 ESTIMATED COST FOR RECEIPT AND LOADING OF SOC OVERPACK, B-20 AND PREPARATION FOR SHIPMENT

10.0 SUMMARY OF ESTIMATED AT-REACTOR COSTS B-20

11.0 REFERENCES FOR APPENDIX B $3-24$ 


\section{APPENDIX B}

ESTIMATED COST OF HANDLING OPERATIONS AT THE REACTOR SITE INVOLVING

SPENT FUEL DRY STORAGE MODULES, TRANSPORT CASKS, AND SOC OVERPACKS

There are a number of costs that would be associated with the handling of TSCS, SOCS, concrete storage caskS, DOE-furnished transport casks, and overpacks for socs (assuming that socs would be shipped in overpacks), at the reactor site. In this Appendix estimates of the costs associated with the at-reactor handling of TSCS, SOCS and concrete storage casks and their contents have been developed, along with estimates of the costs involved for receipt, loading and shipping of DOE-furnished transport casks, and overpacks for Socs.

The various operations involved with the handling of TSCS, SOCS, and concrete storage casks at the reactor site are summarized in the flowsheets shown in Figure $B-1$ and $B-2$. Figure $B-1$ shows the operations associated with the use of TSCS as well as SOCs; three different options have been shown for the use of socs including:

- unloading of SOCs and loading of the contents thereof into DOE-supplied shipping casks

- direct shipment of SOCs to DOE (use in a single transport)

- direct shipment of SOCS to DOE after placing the SOC in a NRCapproved overpack

Figure B-2 shows the operations associated with the use of concrete storage casks.

The cost for the various handling operations described in Figures B-1 and B-2 were estimated in 1987 dollars. In developing these cost estimates for operations involving metal casks (TSCS, SOCS and DOE-furnished transport casks), the study made use of previous work performed by Pacific Northwest Laboratories (PNL) which included a detailed time and motion analys is of the activities involved in the receipt, loading, decontamination, testing and shipment of transport casks (Reference (1)), many of which are applicable to the use of TSCS and SOCs. In developing cost estimates for the 


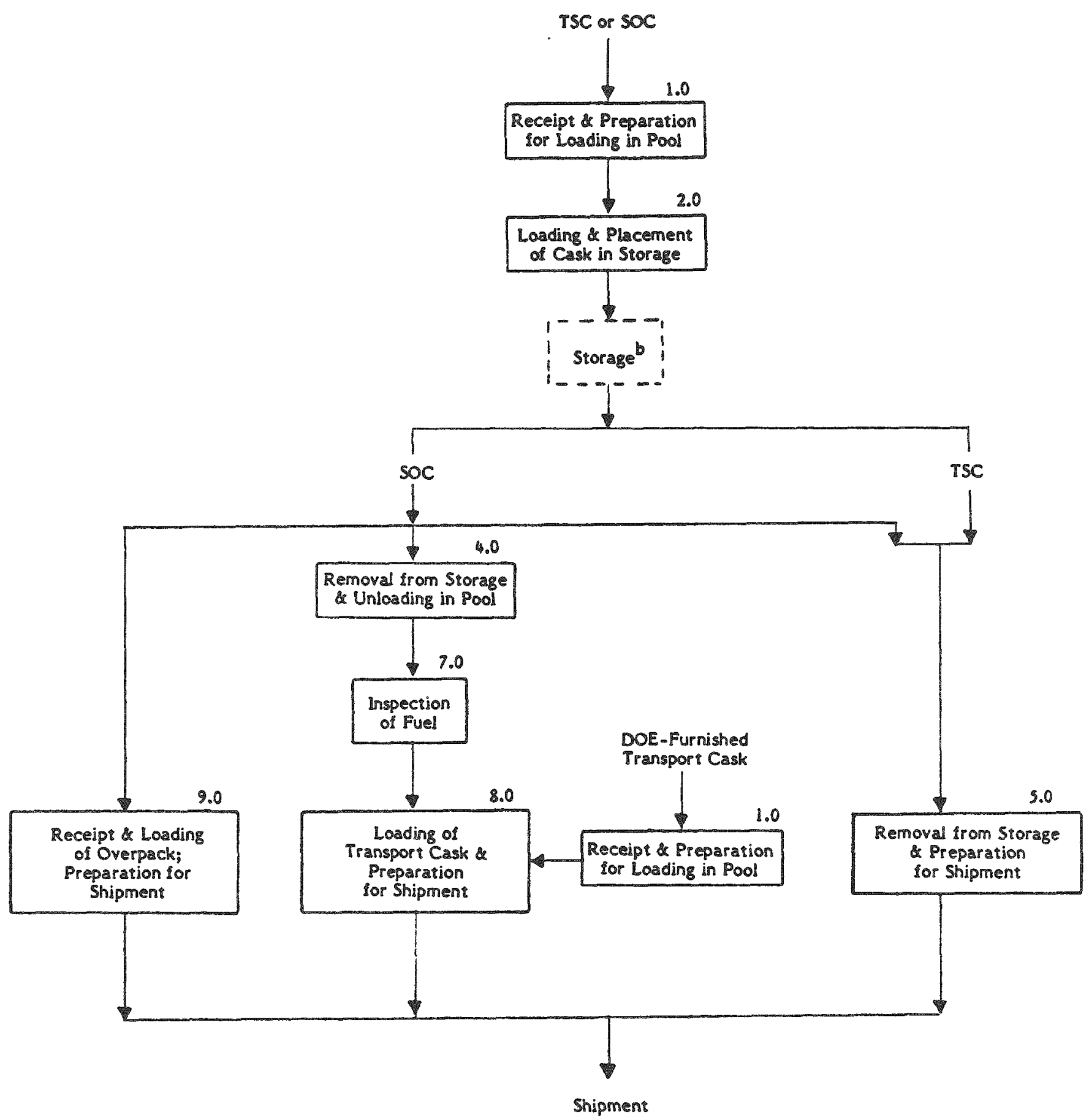

- Numbers on upper right hand corner of box are Section numbers in Appendix $B$ in which costs are developed.

b Costs associated with storage were not included in this estimate.

FIGURE -1

SUMMARY OF OPERATONS ASSOCIATED WITH THE HANDLING OF METAL STORAGE CASES

ATREATOR STTES

B-2 


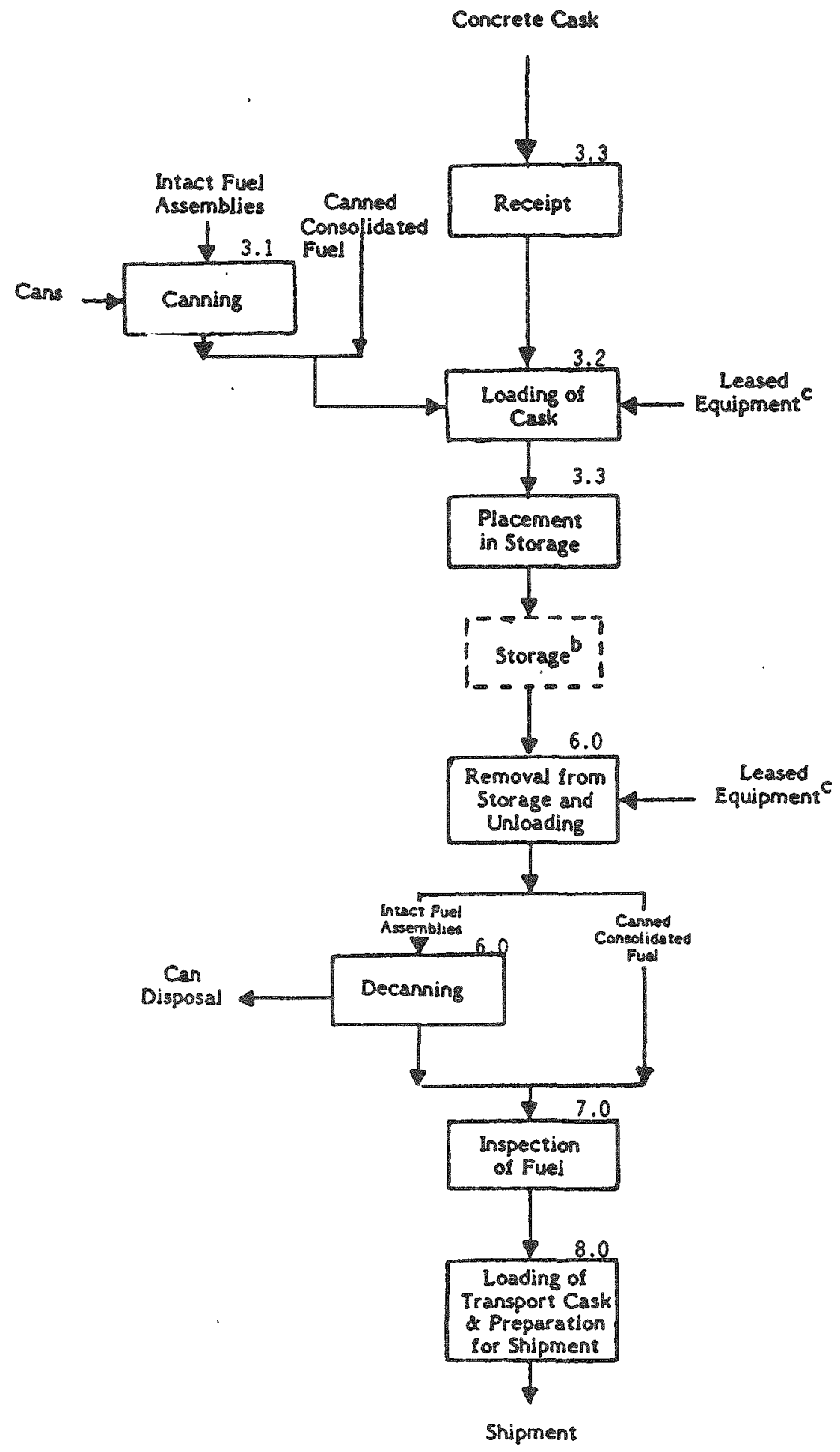

2

Numbers on upper right hand comer of box are Section numbers in Appendix $B$ in which costs are developed.

b

Costs associated with storage were not included in this estimate.

Part of the equipment necessary for loading of casks was leased and the cost thereof was included in these estimates.

FIGURE $2-2$

SUMMARY OF OPERATIONS ASSOCIATED WITH THE HANDLNG OF CONCRETE STORAGE CASKS AT REACTOR SITES

$B-3$ 
canning of spent fuel, inspection, and loading/unloading of a concrete storage cask, the study made use of previous work performed by $E$. R. Johnson Associates, Inc. (JAI) for PNL as well as the experience which has been gained recently at Three Mile Island in the dry loading of canisters of irradiated fuel pieces into a shipping cask. In developing cost estimates for the loading of an overpack containing a SOC onto the transport vehicle, the study made use of the 1986 FIS Fee Study (Reference (2)) for time requirements for riggers as well as crane rental costs.

In addition to the foregoing, the following assumptions formed the basis for the cost estimates developed:

(1) Operating costs were based on an assumed rate of $\$ 63 /$ hour for plant operators, which covered the cost of wages, supervision, overhead and operating supplies.

(2) The capacity of TSCS, SOC; and DOE-furnished transport casks was assumed to be 21 PWR assemblies or 46 BWR assemblies, or the same number of cans of consolidated spent fuel.

(3) The capacity of concrete storage casks was assumed to be 9 PWR assemblies or 25 BWR assemblies, or the same number of cans of consolidated spent fuel.

(4) Where consolidated spent fuel was involved, it was assumed that consolidation of 10 fuel assemblies would result in 5 cans of fuel rods and 1 can of compacted structural parts, with each can having a slightly larger cross section than the fuel assembly from which the consolidated fuel originated (about 9inches $\times 9$-inches for PWR fuel and 6-inches $\times 6$-inches for BWR fuel).

(5) The reactor has a capability for handling a 120-ton loaded cask. (If a reactor does not have such a capability, a costly dry transfer system would be required.)

The following sections describe the details of the cost estimates that were made.

1.0 ESTIMATED COST OF RECEIPT OF DOE-FURNISHED TRANSPORT CASKS OR METAL STORAGE CASK AND PREPARATION FOR LOADING

The various steps involved in the receipt of a transport cask, TSC or SOC, placing it in the reactor pool, and preparing it for the loading of spent fuel assemblies or cans, is shown in Figure B-3, along with the 
125 person-minutes

1 Receve Transport Vehucle an Empy Cask - Montor - Inspect Detach Carner's Dive Unit-

Antach Utwty Drve Unit

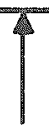

Transport Cask

TSC or SOC
175 person-munute:

2 Move Transport Vehicie and

Cask to inspection and

Washdown Area

Wash Transport Vehcle and

Cask - Monitor - Inspect

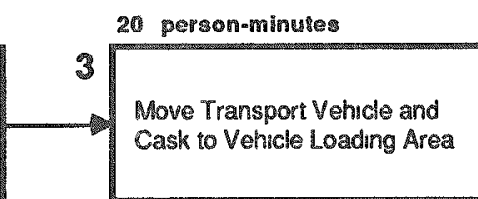

Cask to Vehicle Loading Area
405 person-minutes

Prepare Cask for Piemoval from Transport Vehicle - Remove Impact Limiters and Tiedowns

Remove Cask from Vehucle and Place on Cask Service Pad Remove Transport Vehicle from Loading Area

Prepare Cask for Placement in Loading PIt - Remove Outer Lid Losen Inner Lid - Add

Contamination Shroud

COST PER ASSEMBEY OF ACTIVITY

PWR - \$96.3/Assembly or can BWR - \$45.3/Assembiy or can
TOTAL (person-minutes)

PWR - 1925 person-minutes

BWR - 1985 person-minutes
Prepare Cask for Loading Remove Inner Lid - Inspect and Clean Inner Cavity

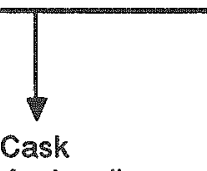

Ready for Loading

\section{Figure $B-3$}

Operations To Receive And Prepare Transport Casks Or Metal Storage Casks

For Loading Spent Fuel At The Reactor Site 
corresponding operating labor time requirements. At $\$ 63 /$ person-hour the cost amounts to $\$ 2,021$ for a cask for PWR fuel ( $\$ 96.3 /$ assembly or can), and $\$ 2,084$ for a cask for BWR fuel ( $\$ 45.3 /$ assembly or can). This represents an average of $\$ 0.22 / \mathrm{kgu}$ contained in fuel assemblies, based on an assumed mix of two thirds of the weight of uranium handled being in the form of PWR fuel assemblies and containing $461 \mathrm{kgU}$, and one third of the weight of uranium handled being in the form of BWR assemblies and containing $183 \mathrm{kgU}$. For consolidated fuel the average cost is $\$ 0.13 / \mathrm{kgU}$.

2.0 ESTIMATED COST OF LOADING A TSC OR SOC AND PLACEMENT IN STORAGE

The various steps involved in removing the spent fuel from the storage pool, loading it into TSCS or SOCs, removal of the cask from the pool, and transferring it to the storage area at the reactor site, are shown in Figure $\mathrm{B}-4$, along with the corresponding operating labor time requirements. The costs involved amount to $\$ 3,392$ for a cask loaded with PWR fuel ( $\$ 161.5$ /assembly or can), and $\$ 5,423$ for a cask loaded with BWR fuel $(\$ 117.9 /$ assembly or can). This represents an average of $\$ 0.45 / \mathrm{kgU}$ contained in intact fuel assemblies (assuming the same mix and weights set forth in Section 1.0 of this Appendix $B$ ), and an average of $\$ 0.27 / \mathrm{kgU}$ for consolidated fuet. 
1575 person-minules PW 3450 person-minutes Bwn

1 Move Spent Fuel Assemblies

from Storage Pool to the

Loading Pit - Place Spent Fuel

Assemblies in Cask

70 person-minutes

2

Intall Fuel Spacers and Imner Lid on the Shipping Cask
185 person-minutes

3 Lif Cask from Loading Pil - Instal inner Lid Bolts and Drip Diaper and Place on Service Pad
750 mersen-minutes

go peron-minutes

Prepare Cask for Movement
Storage - Install Lids - Flush,

Drain and Dy Cask - Penove

Contamination Shroud - Seal

Cask

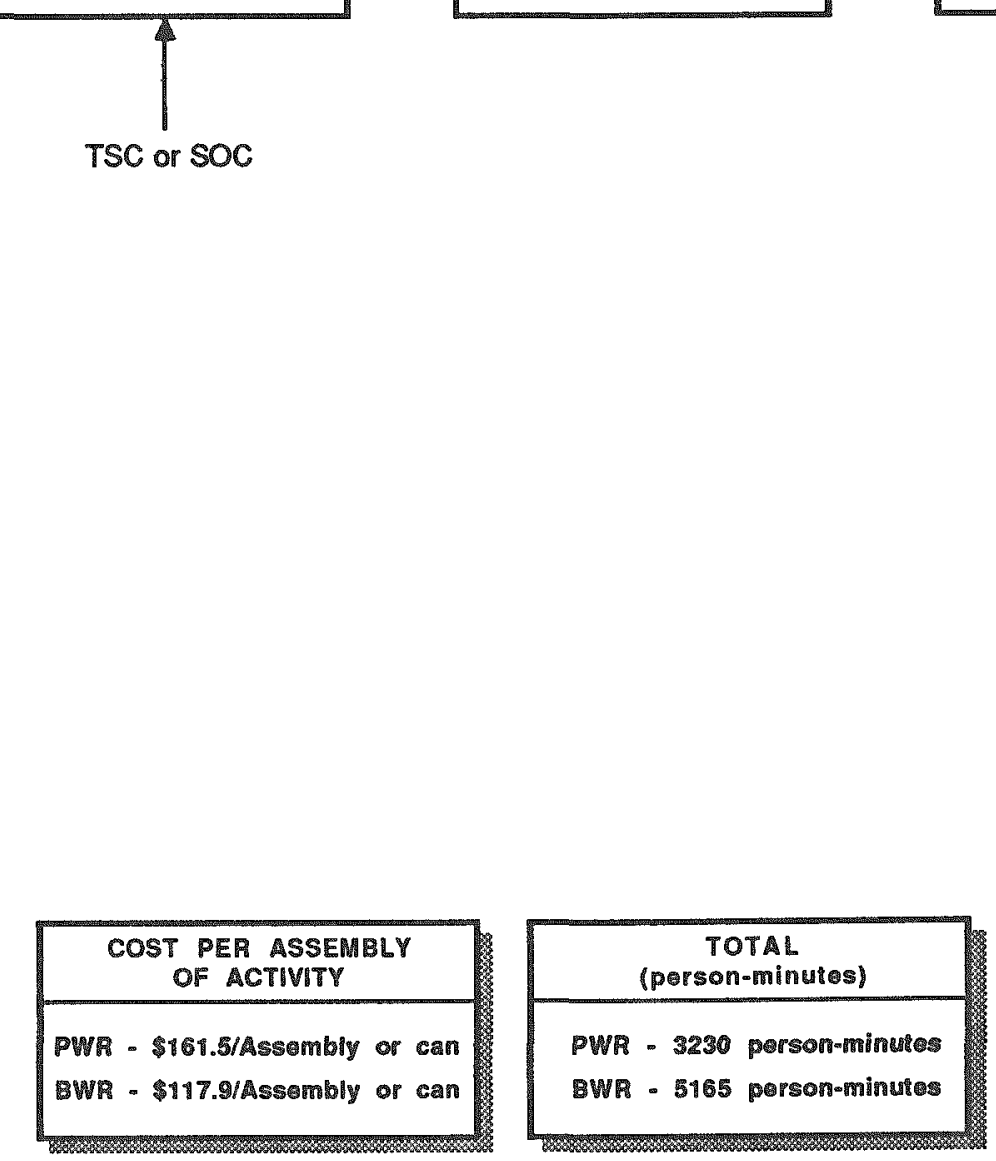

Figure B-4

Operations To Transfer Spent Fuel From The Fuel Pool To A TSC Or SOC

And Move The Cask To The Dry Storage Area 
In developing the estimated costs for loading a concrete cask and placing it in storage, the following assumptions were made:

(1) the concrete cask would be loaded vertically

(2) the cask could not be loaded in the reactor pool but would rather have to be loaded outside the pool using a transfer cask

(3) the cask would be brought from a nearby fabrication location, loaded close to the reactor building and heavy-hauled to its storage pad

(4) the spent fuel would have to be canned prior to placing it in the cask.

The following sections describe the estimated cost for cask loading and placement in storage.

\subsection{ESTIMATED COST OF CANNING}

The estimated capital costs associated with a canning station for spent fuel assemblies or for consolidated fuel are set forth in Table B-1.

\section{TABLE B-1}

ESTIMATED CAPITAL COST FOR CANNING STATION EQUIPMENT

$$
(\$ 000,1987)
$$

Description

Canister Rack

Jib Hoist

Tools

Purge System

Inert Gas Backfill System

Modifications to Pool

Equipment Installation

Engineering

Contingency (15\%)

Total
Cost

$\$ 7.3$

10.3

13.3

25.8

4.5

61.2

22.1

29.2

112.5

13.6

126.1

18.9

$\$ 145.0$ 
These costs were derived from estimates developed in Reference (3) and escalated to 1987 using the Machinery and Equipment index. These captial costs would be incurred by a utility separately from the operating costs otherwise estimated in this Appendix B, Section 3.0 .

The estimated cost of canisters for canning of spent fuel assemblies and consolidated fuel are set forth in Table B-2.

TABLE B-2

\section{ESTIMATED COST OF CANISTERS}

(1987 Dollars)

\begin{tabular}{lrr} 
Description & \multicolumn{1}{c}{ Cost } \\
Materials & $\$ 778$ \\
SS Sheet \& Plate & 83 & \\
Flange Nuts \& Bolts & 156 & \\
Purge Lines \& Fittings & 80 & \\
Gasket \& Miscellaneous & & \\
Fabrication & 1,555 & \\
Can \& Base Plate & 363 & \\
Top Plate & 156 & \\
Flange & & $\$ 3,370$ \\
Quality Assurance & & $\$ 3,876$ \\
\hline
\end{tabular}

These costs were also derived from estimates developed in Reference (3) and escalated to 1987 . These costs represent an average of $\$ 9.03 / \mathrm{kg}$ contained in intact fuel assemblies (assuming the same mix and weights set forth in Section 1.0 of this Appendix 3 ). No incremental cost of canisters is required for consolidated fuel inasmuch as it would have already been canned as part of the consolidation process.

The labor requirements for canning were estimated in Reference (3) to be 3.4 person hours per canister. Thus, the costs involved amount to $\$ 223 /$ canister, including an allowance for incremental utility costs. This 
cost represents an average of $\$ 0.52 / \mathrm{kgU}$ contained in intact fuel assembies (assuming the same mix and weights set forth in Section 1.0 of this Appendix B).

\subsection{ESTIMATED COST OF LOADING CONCRETE CASKS}

Members of the project team involved in this study visited the Three Mile Island (TMI) nuclear power plant to observe the transfer of canisters of recovered fuel rubble from Unit No. 2 from the spent fuel storage pool to a transport cask located outside of the pool, inasmuch as this involved the same type of operations visualized for loading the concrete casks. During the course of the visit it was learned that TMI had been successful in performing a single transfer operation in 2 hours using a crew of 10 operators. While this is considered to be better than average performance by TMI, for the purposes of this study it was considered to be achievable in routine operations involving intact spent assemblies or consolidated fuel which did not involve transfer of highly contaminated items such as that involved at TMI.

Thus, the labor requirements for the transfer of a can containing 1 PWR assembly, 2 BWR assemblies, or consolidated fuel from a reactor storage pool to a concrete storage cask was estimated to be 20 person hours; the costs involved amount to $\$ 1,300 /$ canister, including an allowance for incremental utility costs. These costs represent an average of $\$ 3.03 / \mathrm{kgU}$ contained in intact fuel assemblies (assuming the same mix and weights set forth in section 1.0), and an average of $1.70 / \mathrm{kgU}$ for consolidated fuel.

3.3 ESTIMATED COST OF RECEIPT OF CONCRETE CASK, CLOSURE AFTER LOADING, AND PLACEMENT IN STORAGE

The various steps involved in receiving the concrete cask at the reactor site, closure and inspection after loading, and transfering it to the storage area, are shown in Figure B-5 along with the corresponding operating labor time requirements. The costs involved amount to $\$ 1,985 /$ cask. This cost represents an average of $\$ 0.46 / \mathrm{kgU}$ contained in intact fuel assemblies (assuning the same mix and weights set forth in Section 1.0 of this Appendix $B)$, and an average of $\$ 0.29 / \mathrm{kgu}$ for consolidated fuel. 


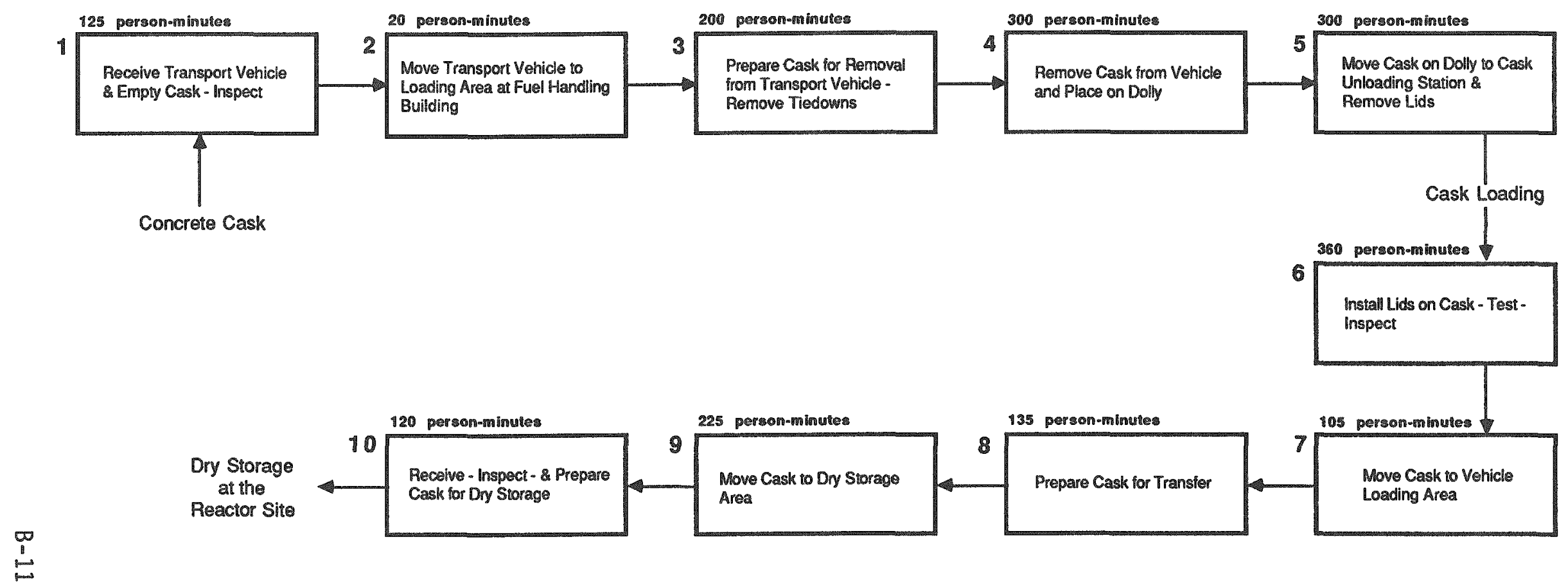

\begin{tabular}{|c|}
\hline $\begin{array}{c}\text { COST PER ASSEMBLY } \\
\text { OF ACTIVITY }\end{array}$ \\
\hline PWP - \$210/Assombly or can \\
BWR - \$75.6/Assembly or can
\end{tabular}

\begin{tabular}{|c|}
\hline $\begin{array}{c}\text { TOTAL } \\
\text { (person-minutes) }\end{array}$ \\
\hline 1890 person-minutes \\
\hline
\end{tabular}

Figure B-5

Operations To Receive Concrete Cask, Close After Loading,

And Move The Cask To The Dry Storage Area 
4.0 ESTIMATED COST OF REMOVAL OF A SOC FROM STORAGE AND UNLOADING IN REACTOR POOL

The various steps involved in removal of a SOC from the dry storage area at the reactor site, placing it in the unloading pool, and unloading the contents thereof, are set forth in Figure B-6, along with the corresponding operating labor time requirements. The costs involved amount to $\$ 2,388$ for a cask loaded with PWR fuel (\$113.8/assembly or can), and $\$ 3,239$ for a cask loaded with BWR fuel $(\$ 70.4 /$ assembly or can). This represents an average of $\$ 0.29 / \mathrm{kgU}$ contained in intact fuel assemblies (assuming the same mix and weights set forth in Section 1.0 of this Appendix B), and an average of $\$ 0.18 / \mathrm{kgU}$ for consolidated fuel.

5.0 ESTIMATED COST OF REMOVAL OF A TSC FROM STORAGE AND PREPARATION FOR SHIPMENT

The various steps involved in removal of a TSC from the dry storage area at the reactor site, and preparing it for shipment to $D O E$, are set forth in Figure B-7 along with the corresponding operating labor time requirements. The costs invoived amount to $\$ 1,150$. This represents an average of $\$ 0.12 / \mathrm{kgU}$ contained in intact fuel assemblies (assuming the same mix and weights set forth in Section 1.0 of this Appendix B), and an average of $\$ 0.08 / \mathrm{kgU}$ for consolidated fuel. 


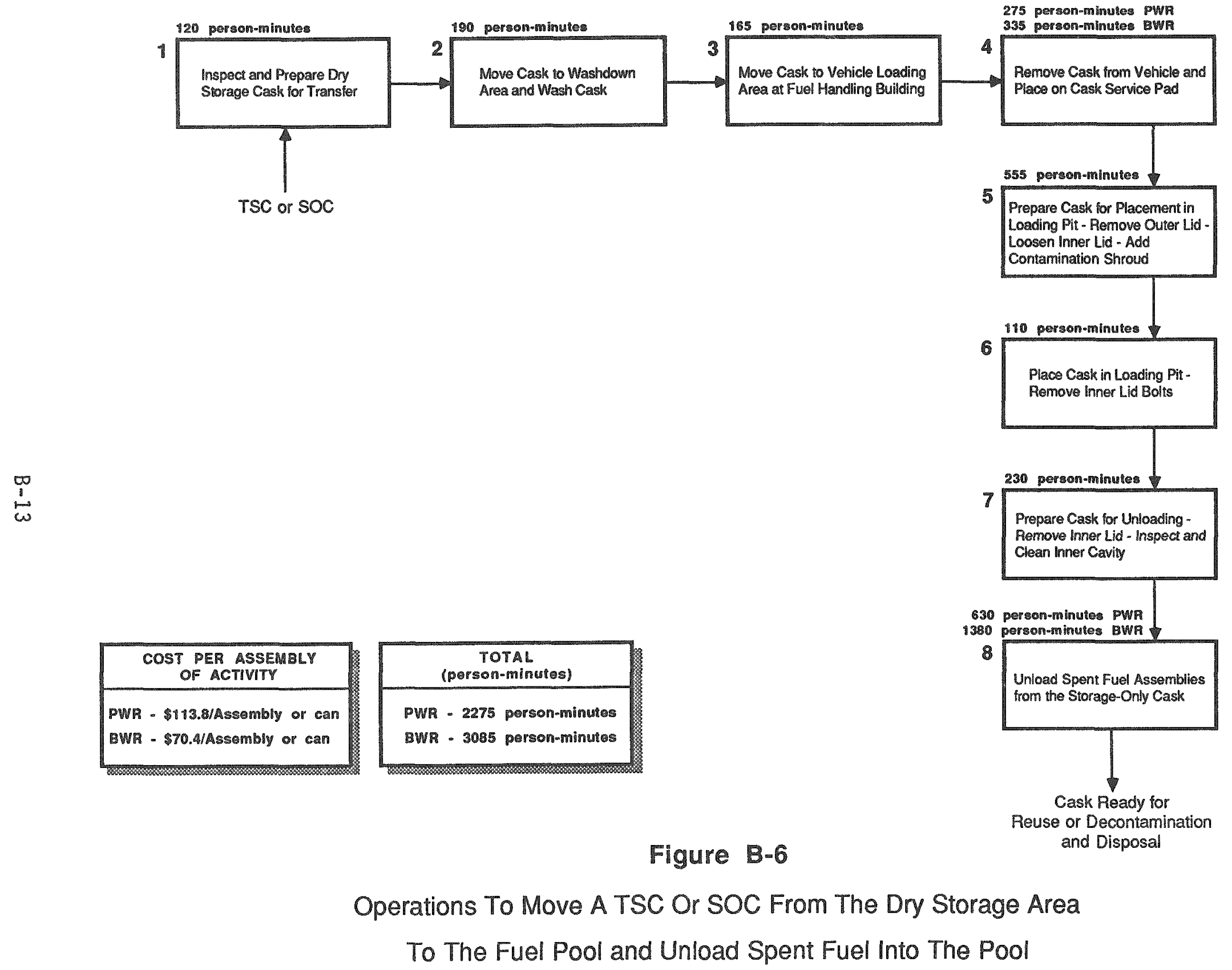




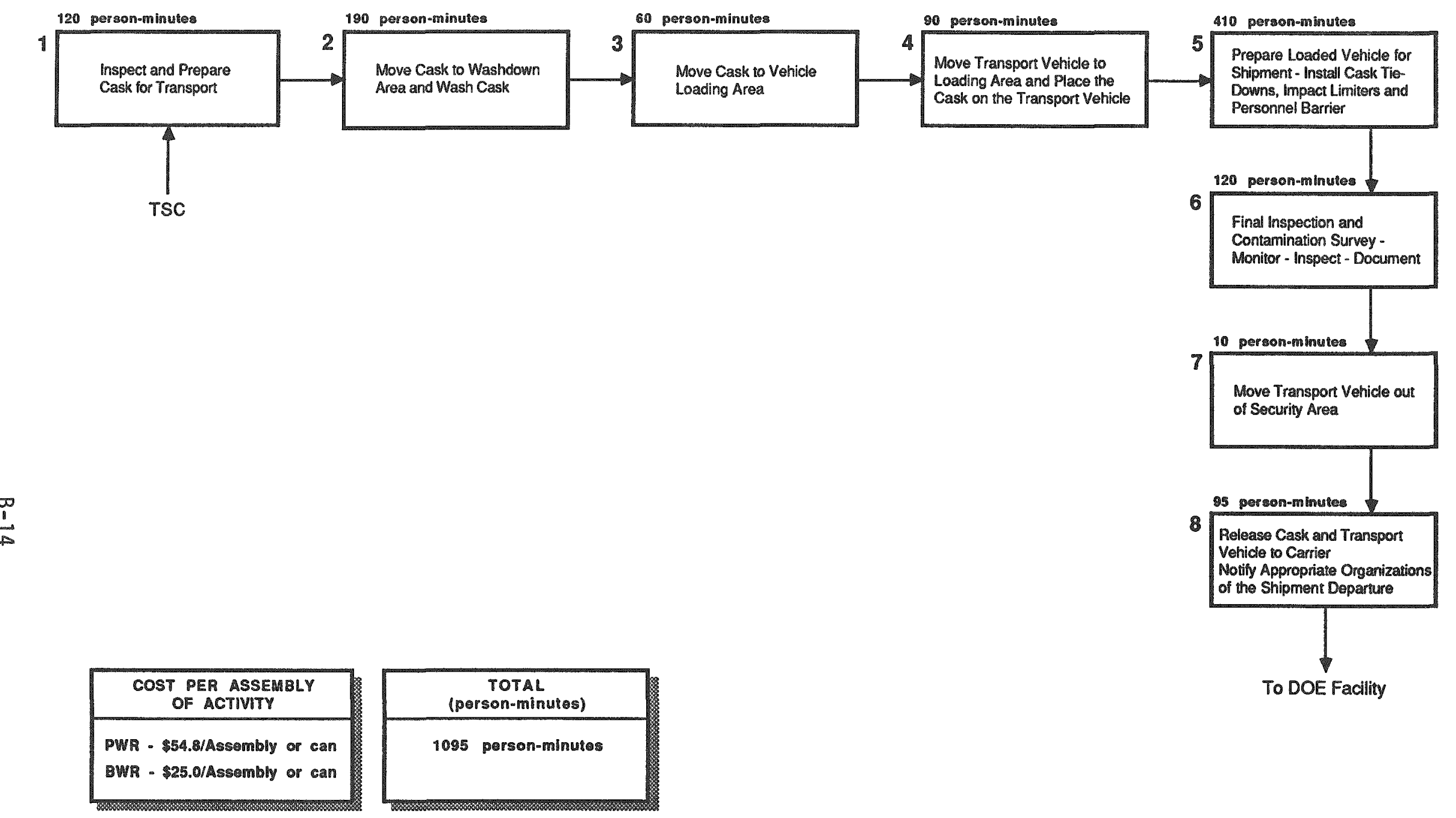

Figure $B-7$

Operations To Remove TSC From Storage And

Prepare For Shipping From The Reactor Site 

UNLOADING

The various steps involved in removal of a concrete cask from the dry storage area at a reactor site are shown in Figure B-8. The costs involved amount to $\$ 929 /$ cask. This represents an average of $\$ 0.22 / \mathrm{kgU}$ contained in intact fuel assemblies (assuming the same mix and weights set forth in Section 1.0 of this Appendix B), and an average of $\$ 0.13 / \mathrm{kgU}$ for consolidated fuel.

The cost of unloading cans from the concrete casks and the removal of the fuel from the cans was assumed to be the same as for loading the casks (see Section 3.2 of this Appendix B) and canning the fuel (see Section 3.1 of this Appendix B), respectively. of course, in this instance there would be no cost for canisters nor any capital costs for equipment since it would have been purchased earlier in connection with the canning operation. The leased equipment described in Section 3.0 of Appendix A (\$750-thousand for a portion of the transfer equipment) would be required again, and the cost thereof incurred during unloading of the concrete casks. This amounted to $\$ 1.45 / \mathrm{kg} U$ for intact fuel assemblies and $\$ 1.26 / \mathrm{kgU}$ for consolidated fuel.

However, it was assumed that the cans used for intact fuel would have to be disposed of after use. The cost of disposal of cans was estimated as follows:

(1) It was estimated that a canister had about $0.5 \mathrm{cu} . \mathrm{ft}$. volume of metal and that it could be compacted so that when packaged the net packing efficiency would be 50 percent.

(2) It was assumed that each canister would be capable of holding 1 PWR assembly or 2 BWR assemblies.

(3) It was estimated that the cost of crushing and packaging a can would amount to $\$ 250$ and that the cost of shipping to a disposal site would amount to $\$ 200$. 


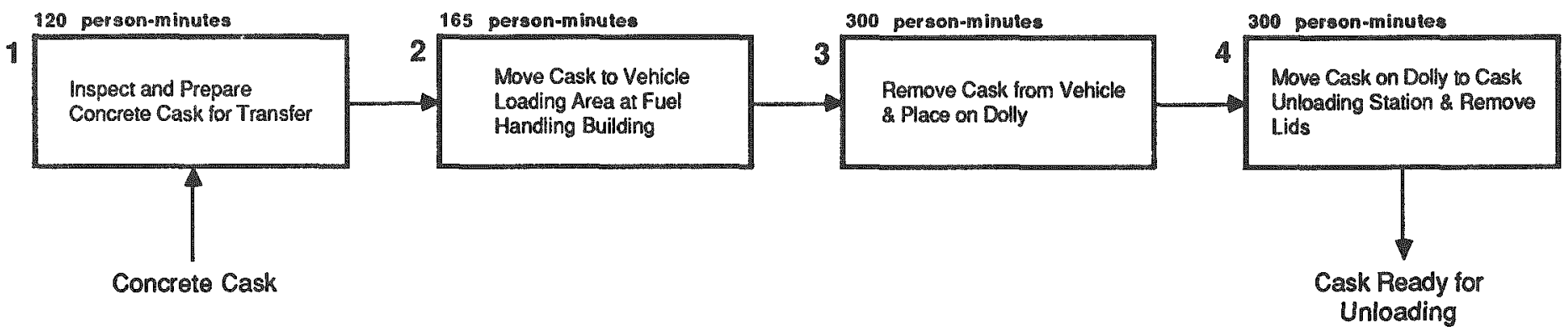

\begin{tabular}{|c|c|}
\hline $\begin{array}{c}\text { COST PER ASSEMBLY } \\
\text { OF ACTVITY }\end{array}$ & $\begin{array}{c}\text { TOTAL } \\
\text { (person-minuies) }\end{array}$ \\
\hline $\begin{array}{l}\text { PWR - \$103/Assembly or can } \\
\text { BWR - \$37/Assembly or can }\end{array}$ & 885 porson-minutes \\
\hline
\end{tabular}

\section{Figure B-8}

Operations To Move A Concrete Cask From The Dry Storage Area

To The Fuel Handling Building 
(4) It was estimated that the cost of disposal of a can at a low level waste burial site would be $\$ 40$, based on Chem-Nuclear systems, Inc. schedule of charges for 1987 (Reference (4)). This is based on an average of 1 cubic foot/can and the following rates:

Standard Waste

Extended Care Fund

South Carolina

$\$ 31.50 / \mathrm{cu} . \mathrm{ft}$.

Low Level Radioactive

2.80

4.00

Waste Disposal Tax

Southeast Regional

Compact Fee

Barnwell County Business

License $\operatorname{Tax}(2.4 \%)$

Total

$\$ 40.08 / \mathrm{cu} . \mathrm{ft}$.

The above amounts to a total cost of $\$ 490 /$ canister. This represents an average of $\$ 1.15 / \mathrm{kgU}$ contained in intact fuel assemblies (assuming the same mix and weights set forth in Section 1.0 of this Appendix B).

\section{$7.0 \quad$ ESTIMATED COST OF INSPECTION}

It was assumed that in the event it was necessary to remove spent fuel from a TSC, SOC or concrete cask after a long storage period, it would also be necessary to reinspect the fuel prior to shipment to DOE in a DOE-furnished transport cask.

In a recent EPRI study Reference (5), it was estimated that it would require 9.63 person-hours/PWR assembly and 6.74 person-hours/BWR assembly to perform a thorough visual inspection of the corresponding assembly.

It was assumed in this study that only about half of this time requirement would apply to cans of consolidated fuel (for visual examination and pressure testing). Moreover, it was assumed that no capital equipment additions would be required for reinspection inasmuch as the necessary equipment would have already been purchased for the initial inspection. Thus, the costs involved amount to \$607/PWR assembly and \$425/BWR assembly, and $\$ 304 /$ can of consolidated PWR fuel and $\$ 213 /$ can of consolidated BWR fuel. This represents an average of $\$ 1.66 / \mathrm{kgu}$ contained in intact fuel assemblies (assuming the same mix and weights set forth in Section 1.0 of this Appendix B), and an average of $\$ 0.50 / \mathrm{kgU}$ for consolidated fuel. 
The various steps involved in loading spent fuel into a transport cask (or a TSC if such is used as part of the DOE cask fleet), removing it from the cask loading pool, and preparing it for shipment to a DOE site, are shown in Figure B-9, along with the corresponding operating labor time requirements. The costs involved amount to $\$ 3,717$ for a cask loaded with PWR fuel ( $\$ 177 /$ assembly or can), and $\$ 5,748$ for a cask loaded with BWR fuel $(\$ 125 /$ assembly or can). This represents an average of $\$ 0.48 / \mathrm{kgU}$ contained in intact fuel assemblies (assuming the same mix and weights set forth in Section 1.0 of this Appendix B), and an average of $\$ 0.29 / \mathrm{kgU}$ for consolidated fuel. 
1575 person-minutes PWR 3450 person-minutes BWR

1 Move Spent Fuel Assemblies from Storage Pool to the

Loading Pit - Place Spent Fuel

Assemblies in Cask

70 person-minutes
Install Fuel Spacers and
Inner Lid on the Shipping Cask

90 permon-minutes

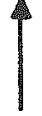

Transport Cask

Litt Cask From Loading Pit Install Inner Lid Bolts and Drip Diaper and Place on Service Pad

4 Prepare Cask for Shipment Install Lids - Flush, Drain and Dr Cack - Pemove Contamination

Shroud - Seal Cask

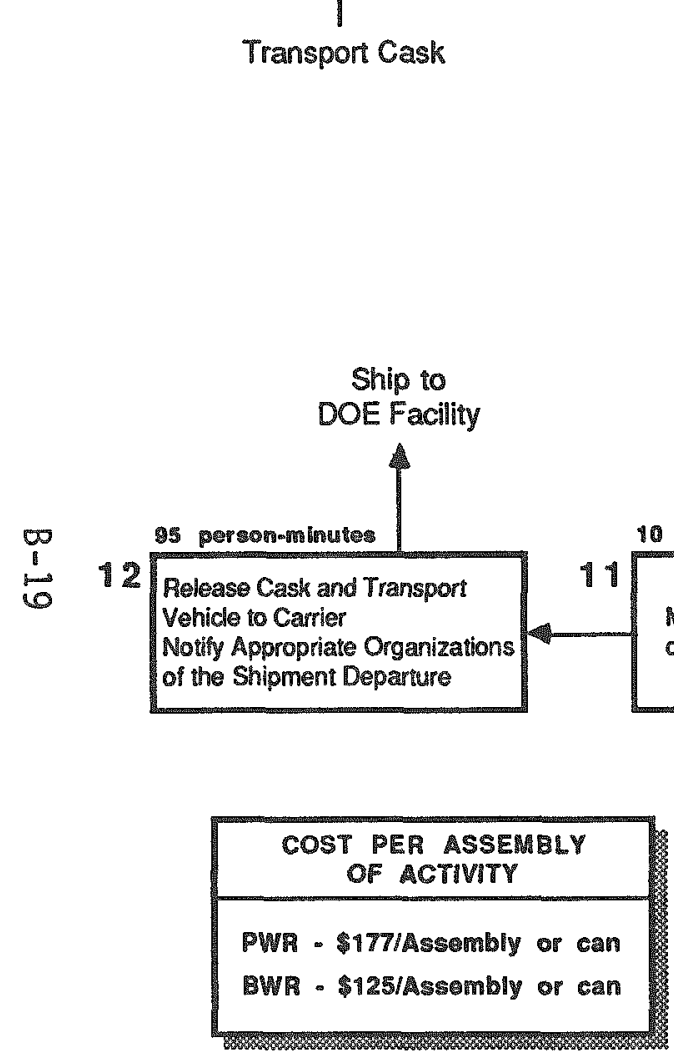

10 person-minutes Move Transport Vehicle out of Security Area

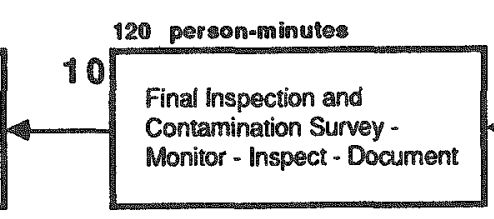

410 person-minutes

Prepare Loaded Vehicle for Prepare Loaded Vehict
Shipment - Install Cask Tiedowns, Impact Limiters and Personnel Barrier

6

Move Cask to Vehiclo Loading Area

75 person-minutes PWR 135 person-minutes

7

Move Transport Vehicle to Loading Area and Place Cas on the Transport Vehicle

Release Cask and Tran

Vehicle to Carrier

f the Shipment Departure

Moniror - Inspect - Document

Figure $B-9$

Operations To Load Spent Fuel In Tranport Casks And

Prepare The Casks For Leaving The Reactor Site

PWR - 3540 person-minutos

BWR - 5475 porson-minutes 
9.0 ESTIMATED COST FOR RECEIPT AND LOADING OF SOC OVERPACK, AND PREPARATION FOR SHIPMENT

The various steps involved in the receipt of an overpack, loading of a SOC into the overpack, and preparing it for shipment to a DOE site, is shown in Figure $B-10$, along with the corresponding operating labor time requirements. At $\$ 63 /$ person-hour the cost amounts to $\$ 3,843 /$ cask, or $\$ 183 /$ assembly or can for PWR fuel and $\$ 83.5 /$ assembly or can for BWR fuel. In addition crane rental costs for each such loading are estimated to amount to $\$ 3,282$ ( $\$ 1,641 /$ day, 2 days minimum renta1), or $\$ 156.3 /$ assembly or can of PWR fuel and $\$ 71.3 /$ assembly or can for BWR fuel. This represents an average total cost of $\$ 0.77 / \mathrm{kgU}$ contained in intact assembiies (assuming the same mix and weights set forth in Section 1.0 of this Appendix $B$ ), and an average of $\$ 0.46 / \mathrm{kgU}$ for consolidated fuel.

10.0 SUMMARY OF ESTIMATED AT-REACTOR COSTS

A summary of the unit operating costs developed in Sections 1.0 through 9.0 of this Appendix $B$ is set forth in Tables B-3 and B-4 for intact fuel assemblies and consolidated fuel, respectively. 


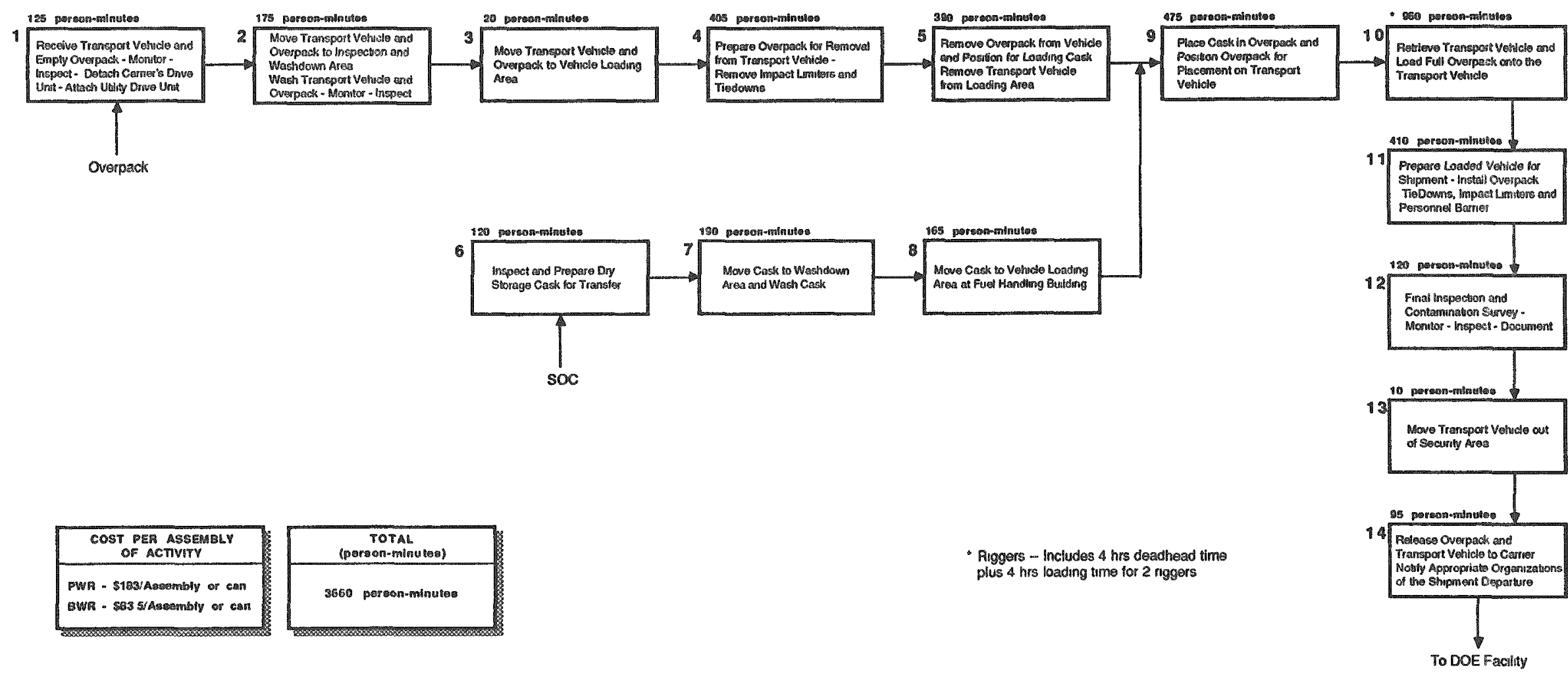

Figure B-10

Operations To Remove A SOC From Storage, Receive An Overpack

And Prepare For Shipping From The Reactor Site 
TABLE B-3

SUMMARY OF ESTIMATED AT-REACTOR HANDLING COSTS ASSOCIATED WITH ORY STORAGE MODULES

(Intact Fuel Assembiles)

\begin{tabular}{|c|c|c|c|c|}
\hline \multirow[b]{2}{*}{$\begin{array}{l}\text { Section } \\
\text { Reference }\end{array}$} & \multicolumn{4}{|c|}{ Average Cost $(\$ / \mathrm{kgu}, 1987)^{\mathrm{a}}$} \\
\hline & $\begin{array}{c}\text { TSC or SOC } \\
\text { Destined For } \\
\text { Shipment } \\
\text { To DOE }\end{array}$ & $\begin{array}{c}\text { SOC } \\
\text { Shipped To } \\
\text { DOE In } \\
\text { Overpack }\end{array}$ & $\begin{array}{c}\text { soc } \\
\text { Used For } \\
\text { At-Reactor } \\
\text { Storage only }\end{array}$ & $\begin{array}{c}\text { Concrete } \\
\text { Cask }\end{array}$ \\
\hline
\end{tabular}

(1) Loading \& Placement in AR Storage

\begin{tabular}{|c|c|c|c|c|c|}
\hline $\begin{array}{l}\text { (a) Cask Receiving \& Placement in Pool } \\
\text { (b) Cans } \\
\text { (c) Canning } \\
\text { (d) Loading \& Transfer to Storage } \\
\text { (e) Equipment Rental }\end{array}$ & $\begin{array}{l}1.0 \\
3.1 \\
2.0,3.2,3.3 \\
\text { App A, 3.0 }\end{array}$ & $\begin{array}{r}\$ 0.22 \\
- \\
0.45 \\
- \\
\end{array}$ & $\begin{array}{r}\$ 0.22 \\
- \\
0.45 \\
\end{array}$ & $\begin{array}{r}\$ 0.22 \\
- \\
0.45 \\
=\end{array}$ & $\begin{array}{l}9 . .^{c} \\
0.53 \\
0.49 \\
3.49 \\
1.45 \\
\end{array}$ \\
\hline Subtotal & & 0.67 & 0.67 & 0.67 & 14.49 \\
\hline
\end{tabular}

(2) Removal From AR Storage o Shipment Preparation
(a) Removal from Storage \& Unloading
(b) Decanning
(c) Can Disposal
(d) Fuel Inspection
(e) Receiving of Transport Cask
f) Loading of Transport Cask
(g) Equi pment Rental
(h) Preparation of TSC or SOC for Shipment
(i) Overpacking of SOC for Shipment

$4.0,6.0$
6.0
6.0
7.0
1.0
8.0
6.0
5.0
9.0
Subtota?

Total

\begin{tabular}{rrrr}
- & - & 0.29 & 3.25 \\
- & - & - & 0.52 \\
- & - & 1.66 & 1.15 \\
- & - & 0.22 & 0.66 \\
$\vdots$ & - & 0.48 & 0.48 \\
0.12 & - & - & 1.45 \\
\hline 0.12 & 0.77 & - & -1.78 \\
$\$ 0.79$ & 1.44 & $\$ 3.32$ & $\$ 23.22$ \\
\hline
\end{tabular}

\footnotetext{
Based on: $2 / 3$ of the amount ( $\mathrm{kg}$ ) of fuel being PWR fuel, with an average of $461 \mathrm{kgU} / \mathrm{assembly}$, and a cask capacity of 21 assemblies; $1 / 3$ of the amount ( $\mathrm{kgU}$ ) of fuel being BWR fuel, with an average of $183 \mathrm{kgU} / \mathrm{assembly}$, and a cask capacity of 46 assemblies.

- References are to Sections in Appendix B unless otherwise indicated

C Included in the costs shown in $(1)(d)$
} 


\begin{tabular}{ccccc} 
& \multicolumn{3}{c}{ Average Cost $(\$ / \mathrm{kgU}, 1987)^{\mathrm{a}}$} \\
\cline { 2 - 4 } & TSC or SOC & SOC & SOC \\
Destined For & Shipped To Used For & \\
Section b & Shipment & DOE In & At-Reactor & Concrete \\
Reference & To DOE & Overpack & Storage Only & Cask
\end{tabular}

(1) Loading Placement in AR Storage

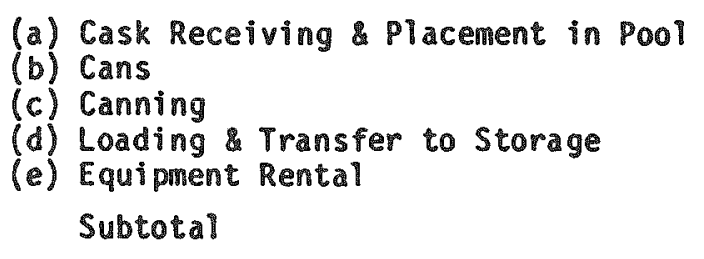

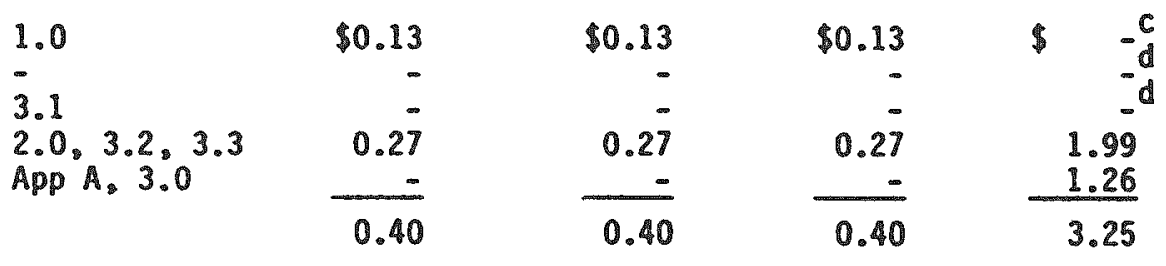

(2) Removal Fron AR Storage \& Shipment Preparation
(a) Removal from Storage \& Unioading
(b) Decanning
(c) Can Disposal
(d) Fuel Inspection
(e) Receiving of Transport Cask
(f) Loading of Transport Cask
(g) Equi pment Rental
(h) Preparation of TSC or SOC for Shi pment
(i) Overpacking of SOC for shipment

4.0 .6 .0
6.0
6.0
7.0
1.0
8.0
6.0
5.0
9.0
Subtotal

Total

\begin{tabular}{|c|c|c|c|}
\hline - & - & 0.18 & 1.83 \\
\hline- & - & - & \\
\hline - & - & - & - \\
\hline- & - & 0.50 & 0.50 \\
\hline - & - & 0.13 & 0.13 \\
\hline & - & 0.29 & 0.29 \\
\hline & - & - & 1.26 \\
\hline 0.08 & - & - & - \\
\hline & 0.46 & $=$ & $=$ \\
\hline 0.08 & 0.46 & 1.10 & 4.01 \\
\hline$\$ 0.48$ & $\$ 0.86$ & $\$ 1.50$ & $\$ 7.26$ \\
\hline
\end{tabular}

\footnotetext{
Based on: 2/3 of the amount ( $\mathrm{kgU}$ ) of fuel being PWR fuel, with an average of $770 \mathrm{~kg} / \mathrm{can}$, and a cask capacity of 21 cans: $1 / 3$ of the amount ( $k g U)$ of fuel being BWR fuel, with an average of $306 \mathrm{kgU} / \mathrm{can}$, and a cask capacity of 46 cans.

References are to Sections in Appendix $B$ unless otherwise indicated

C Included in the costs shown in $(1)(d)$

Consolidated fuel assumed to be canned already
} 
(1) K. J. Schneider et al, Radiation Dose Analys is of a Postulated Reference Transportation System for Commercial Spent Fuel (Draft), Pacific Northwest Laboratories, September 1986.

(2) U. S. Department of Energy, 1986 Federal Interim Storage Fee Study Update: A Technical and Economic Analysis, PNL-6031, September 1986

(3) E. R. Johnson Associates, Inc., Impacts of Certain Spent Fuel Storage Acceptance Criteria, JAI-196, PNL Subcontract No. B-B2473-A-G, October 1982

(4) Chem-Nuclear Systems Inc., Barnwell Low-Level Radioactive Waste Management Facility Rate Schedule, effective January 1, 1987

(5) E. R. Johnson Associates, Inc., Waste Acceptance Criteria Study, (Preliminary Draft), JAI-267, August 26, 1986 
APPENDIX C

ESTIMATES OF LIFE CYCLE AND UNIT COSTS FOR USE OF DRY SPENT FUEL

STORAGE MODULES, TRANSPORT CASKS, AND SOC OVERPACKS

AT REACTOR SITES 
APPENDIX C

ESTIMATES OF LIFE CYCLE AND UNIT COSTS FOR USE OF DRY SPENT FUEL STORAGE MODULES, TRANSPORT CASKS, AND SOC OVERPACKS AT REACTOR SITES

\section{TABLE OF CONTENTS}

1.0 ESTIMATED REQUIREMENTS FOR DRY STORAGE AT REACTOR SITES C-1

2.0 ESTIMATES OF LIFE CYCLE AND UNIT COSTS C -5

3.0 REFERENCES FOR APPENDIX C C- 42 


\author{
APPENDIX C \\ ESTIMATES OF LIFE CYCLE AND UNIT COSTS FOR USE OF DRY SPENT FUEL \\ STORAGE MODULES, TRANSPORT CASKS, AND SOC OVERPACKS \\ AT REACTOR SITES
}

The purpose of this Appendix is to develop estimates of the life cycle costs for the utiljty spent fuel management system that result from the use of TSCS, SOCS and concrete storage casks, as well as the unit costs involved. These costs include the capital costs of the storage modules involved, the capital cost of special equipment required, and the operating costs associated with the handiing of the different storage modules, DOEfurnished transport casks, and SOC overpacks. Specifically excluded from these estimates, however, are the capital cost of storage facilities (storage pad, fencing, security system, monitoring equipment, and the like), and the cost of operation thereof during the storage period. For this purposes of this study such costs were assumed to be the same for the different types of storage modules, even though the cost of storage of concrete casks can be expected to be slightly higher than for TSCS or SOCS. Thus the life cycle and unit costs presented herein do not represent the total costs associated with at-reactor storage by the individual storage modes considered, but rather provide a means of estimating the differences in the costs of the storage methods considered.

\title{
1.0
}

\section{ESTIMATED REQUIREMENTS FOR DRY STORAGE AT REACTOR SITES}

An estimate was first made of the prospective requirements for additional storage at reactor sites and the amount that would be provided by dry storage. This involved the determination of the amounts of storage that :lould be required by utilities in the future in excess of their maximum pool capacity. In this connection the data developed in Reference (1) was used -which involved no new orders and extended fuel burnup. The projected acceptance rate of spent fuel by DOE was subtracted from the annual amounts of storage that would otherwise be needed by the utilities. Three different DOE acceptance rates were considered; these included the 1998 date and spent fuel 
receiving rates set forth in the June 1985 Mission Plan (Reference 2) for the Authorized System, and those involving delays of 5 years and 10 years. The requirements for dry cask storage were developed from the estimates of additional at-reactor storage requirements by evaluating the fraction of such needs that are likely to be met using other technologies. Based on an evaluation of current utility plans, it was estimated that 50 percent of the projected needs for storage would be met by the utilities through consolidation or transshipment, with remaining 50 percent being met by dry storage. Tables $\mathrm{C}-1$ and $\mathrm{C}-2$ show the prospective requirements for dry storage capacity at reactor sites, and the numbers of metal or concrete casks that would be required if either were used alone to meet 50 percent of the projected needs. Table $\mathrm{C}-1$ shows the numbers of storage modules required if all fuel were in the form of intact fuel assemblies, while Table $\mathrm{C}-2$ shows the numbers of modules required if all fuel were in the form of consolidated fuel rods and compacted structural parts. 
TABLE C-B

PROSPECTIVE ORY STORACE MOOULE REQUIREMEMTS FOR AT-REACTOR STORAGE - IMTACT FUEL ASSERBLIES

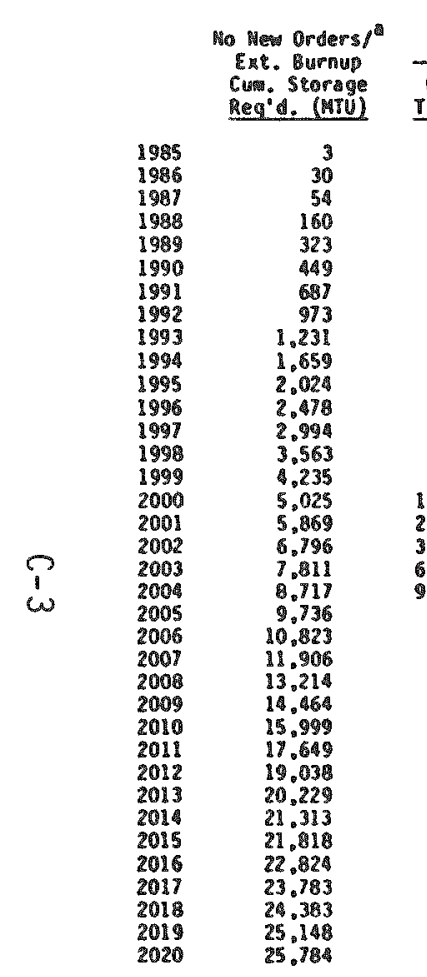

\begin{tabular}{|c|c|c|}
\hline 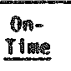 & $\begin{array}{l}\text { S-rr. } \\
\text { Delay }\end{array}$ & $\begin{array}{l}\text { 10-rr. } \\
\text { Delay }\end{array}$ \\
\hline 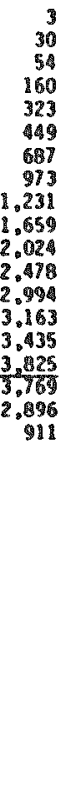 & 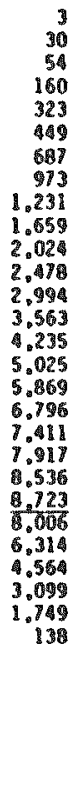 & 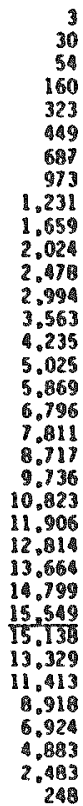 \\
\hline
\end{tabular}
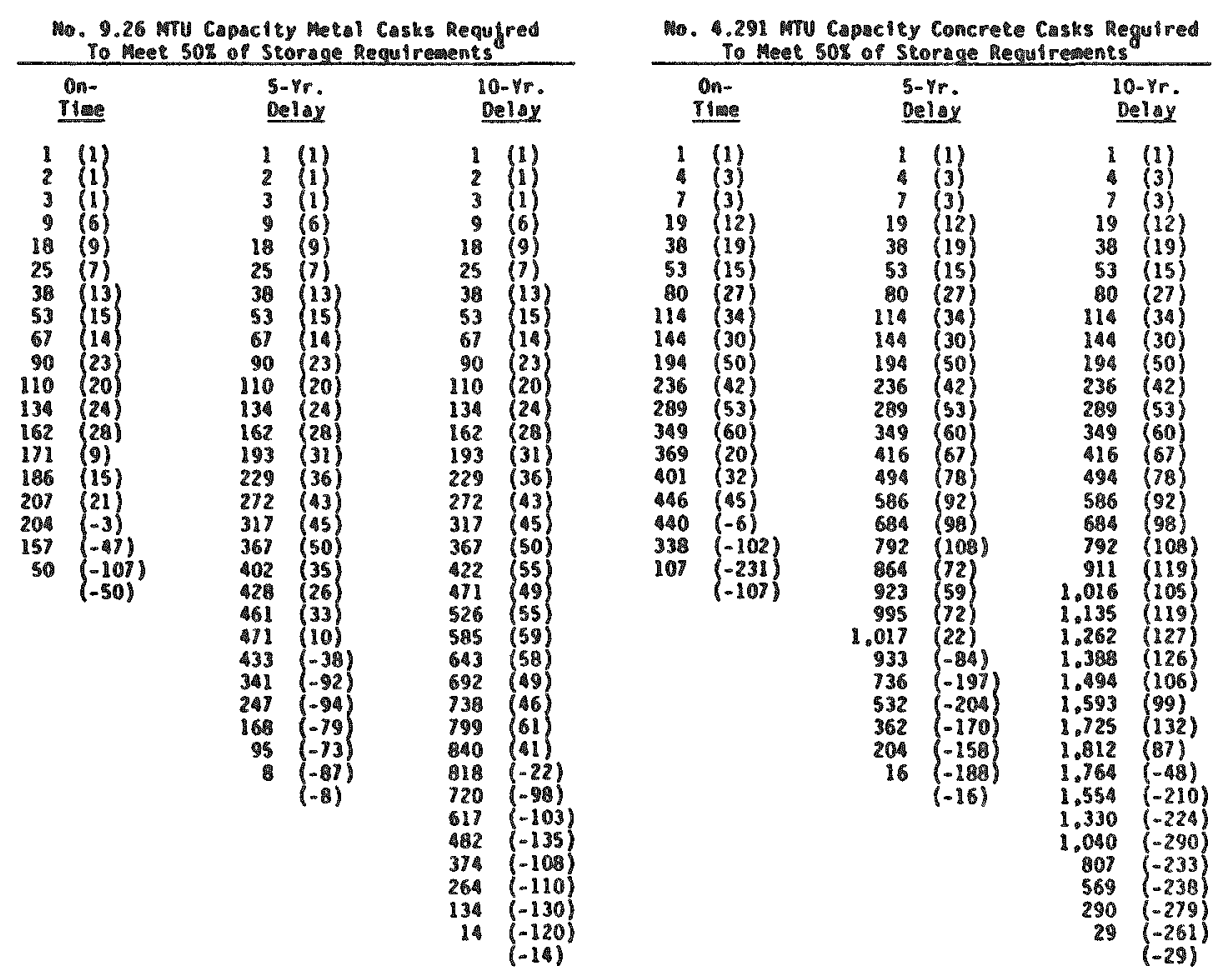

Reference (1): requirements in excess of exiseing capacity

Trom Reference (2)

c Underlined valuas represent the mataum anount of additional at-reactor (AN) storage required for the Individual scenarios Involved

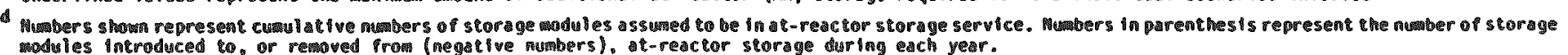


TABLE C-E

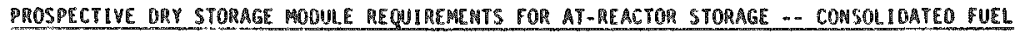

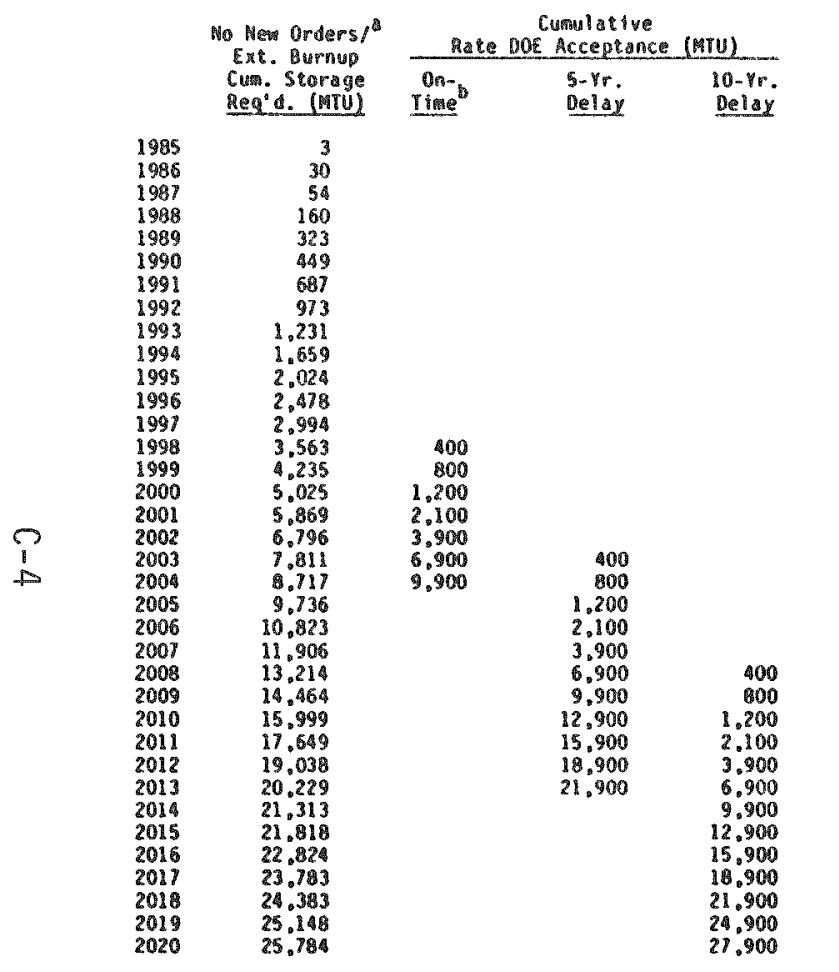

\begin{tabular}{|c|c|c|c|c|c|c|c|c|}
\hline \multicolumn{3}{|c|}{$\begin{array}{l}\text { Cumulaclue Add Itional } \\
\text { An Storage Regutred (ATU) }\end{array}$} & \multicolumn{6}{|c|}{ 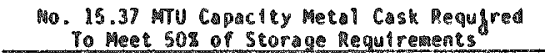 } \\
\hline $\begin{array}{l}\text { On- } \\
\text { Time }\end{array}$ & $\begin{array}{l}\text { 5-rr. } \\
\text { Delay }\end{array}$ & $\begin{array}{l}\text { 10-Pro } \\
\text { Delay }\end{array}$ & \multicolumn{2}{|c|}{$\begin{array}{l}\text { On- } \\
\text { Time }\end{array}$} & \multicolumn{2}{|c|}{$\begin{array}{l}\text { S-ry. } \\
\text { Delay }\end{array}$} & \multicolumn{2}{|c|}{$\begin{array}{l}\text { 10-rr. } \\
\text { Delay }\end{array}$} \\
\hline 3 & 3 & 3 & & (1) & & & 1 & \\
\hline & 30 & 30 & & (i) & & (0) & & (0) \\
\hline 58 & 54 & $\begin{array}{l}58 \\
54\end{array}$ & & (i) & & (i) & $\frac{1}{2}$ & (i) \\
\hline 160 & 160 & 160 & 6 & (is) & & (4) & 6 & (4) \\
\hline 323 & 323 & 323 & II & (5) & & (5) & 11 & (5) \\
\hline 849 & 449 & 449 & & (4) & is & (4) & 15 & (4) \\
\hline 687 & 687 & 681 & & (7) & 22 & (i) & 22 & (7) \\
\hline 973 & $9 / 3$ & 973 & 32 & (10) & 32 & (10) & 32 & (10) \\
\hline 1,231 & 1.231 & 1.231 & 40 & (B) & 40 & (B) & 40 & (a) \\
\hline 1.659 & 1.659 & 1.659 & 58 & (iv) & 54 & (ii) & 34 & (14) \\
\hline 2,024 & 2.024 & 2.024 & & (12) & 66 & (12) & 66 & (12) \\
\hline 2.478 & 2.478 & 2.478 & & (15) & 81 & (15) & 81 & (15) \\
\hline 2.994 & 2.994 & 2.994 & & (17) & 98 & (ii) & 98 & (17) \\
\hline 3,163 & 3,563 & 3.563 & 103 & (5) & 116 & (18) & 116 & (10) \\
\hline 3.435 & 4.235 & 4.235 & 112 & (9) & 138 & (22) & 138 & (22) \\
\hline 38825 & 5.025 & 5.025 & 124 & (12) & 164 & (26) & 104 & (26) \\
\hline 3.586 & 5.369 & 5.869 & 123 & $\{-1\}$ & 191 & (27) & 191 & (27) \\
\hline 2.896 & 6.796 & 6.796 & 96 & $(-29)$ & 222 & (31) & 822 & (31) \\
\hline 911 & 7.411 & 7,811 & 30 & $\left(\begin{array}{r}-64 \\
-30\end{array}\right)$ & $\begin{array}{l}241 \\
258\end{array}$ & (19) & $\begin{array}{l}254 \\
284\end{array}$ & (32) \\
\hline & 8.336 & 9.736 & & & 278 & $\{20\}$ & 317 & (33) \\
\hline & $\begin{array}{l}0.0303 \\
8.723\end{array}$ & $\begin{array}{l}70.823 \\
10\end{array}$ & & & & (6) & 353 & \\
\hline & $\frac{0^{2}}{8.0006}$ & $\begin{array}{l}10.006 \\
11.906\end{array}$ & & & 261 & $\{-23\}$ & 388 & (35) \\
\hline & 6.314 & 12.814 & & & $\begin{array}{l}61 \\
206\end{array}$ & $\left\{\begin{array}{l}-35 \\
-35\end{array}\right.$ & 417 & $(29)$ \\
\hline & 4.564 & 13.664 & & & 149 & $(-58)$ & 445 & (28) \\
\hline & 3.099 & 14.799 & & & 101 & $(-48)$ & 482 & (37) \\
\hline & 1,749 & 15,549 & & & 57 & $(-44)$ & 506 & \\
\hline & $\begin{array}{l}138 \\
138\end{array}$ & 15.138 & & & & $(-52)$ & 493 & $(-13)$ \\
\hline & & 13.329 & & & & $(-5)$ & 434 & -59 \\
\hline & & 11.413 & & & & & 372 & $\mid-62\}$ \\
\hline & & 8.913 & & & & & 291 & \\
\hline & & 6.924 & & & & & 226 & $(-65)$ \\
\hline & & 4,883 & & & & & 159 & $(-67)$ \\
\hline & & 2.483 & & & & & 91 & -78 \\
\hline & & 248 & & & & & 9 & $\left(\begin{array}{l}-72) \\
-91\end{array}\right.$ \\
\hline
\end{tabular}

Mo. 7.12 MTU Capacity Concrese Casks Reguired To Heet 50 os of Storage Requt rements

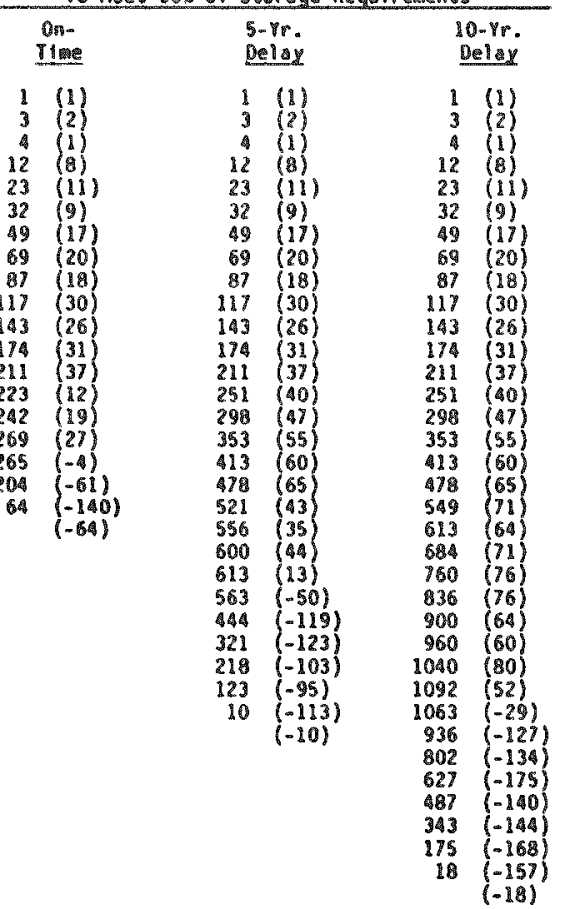

\footnotetext{
a Reference (1): requtrements in excess of existing capacity
}

- Fram Reference (2)

6 Underinned values represent the maximutio amount of additional at-reactor (AR) storage required for the individual scenarios invoived

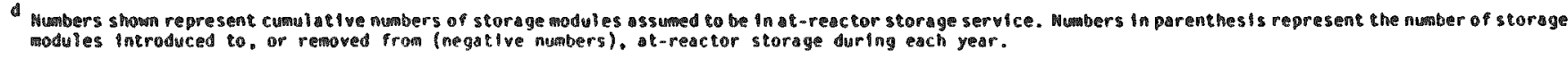


Using the required numbers of storage modules and schedule developed in Section 1.0 of this Appendix C, and the costs of using TSCS, SOCs and concrete storage casks for at-reactor storage as developed in Appendix $A$ and Appendix B, life cycle costs were developed for the following cases:

\begin{tabular}{|c|c|c|}
\hline $\begin{array}{l}\text { Case } \\
\text { No. }\end{array}$ & $\begin{array}{c}\text { Type of } \\
\text { Cask Used } \\
\text { For AR } \\
\text { Storage }\end{array}$ & $\begin{array}{c}\text { Type of } \\
\text { Fuel }\end{array}$ \\
\hline 1 & TSC & Intact assembli \\
\hline 2 & TSC & Consolidated fue \\
\hline 3 & SOC & Intact assemblit \\
\hline 4 & SOC & Consolidated fue \\
\hline 5 & SOC & Intact assemblie \\
\hline 6 & SOC & Consolidated fue \\
\hline 7 & SOC & Intact assembli \\
\hline 8 & $\mathrm{SOC}$ & Consolidated fu \\
\hline 9 & $\begin{array}{c}\text { Concrete } \\
\text { Cask }\end{array}$ & Intact assembli \\
\hline 10 & $\begin{array}{c}\text { Concrete } \\
\text { Cask }\end{array}$ & Consolidated $f$ \\
\hline
\end{tabular}

Method of
Shipment to DOE Facilities

In TSC

In TSC

In DOE-furnished transport cask

In DOE-furnished transport cask

In SOC; one-time use of SOC for shipment

In SOC; one-time use of SOC for shipment

In SOC in overpack

In SOC in overpack

In DOE-furnished transport cask

In DOE-furnished transport cask

For each of the foregoing cases, life cycle costs were developed for three scenarios of deployment of a repository: on-time (1998), 5-year delay (2003), and 10-year delay (2008). These were designated as subcases $A, B$ and $C$, respectively, for each of the cases described above.

The life cycle costs and resulting unit costs for each of the foregoing cases are shown in Tables $c-3$ through $c-32$. Summaries of these costs are shown in Tables $\mathrm{C}-33$ and $\mathrm{C}-34$ for intact fuel assemblies and consolidated fuel, respectively. The figures set forth in the tables were drveloped as follows:

(1) The cumulative amounts of additional fuel to be stored at reactors (AR), in excess of their existing storage capacity, were the same as those shown in Tables $C-1$ and $C-2$. The annual amounts of fuel stored at the reactor represent new additions to storage, and for any given year were determined by subtracting the cumulative amount stored in the preceding year 
from the cumulative amount stored in the given year. Only 50\% of these amounts were assumed to be stored in TSCS, SOCS or concrete casks.

(2) The number of metal casks or concrete casks shown in the tables represent the number of casks introduced to at-reactor (AR) storage in each year. (The number of casks removed from storage are shown in parentheses.) These were determined by dividing the number of MTU introduced to storage annually by the capacity of the corresponding cask - for metal casks the capacities were assumed to be 9.26 MTU/cask for intact fuel assemblies and 15.37 MTU/cask for consolidated fuel; for concrete casks the capacities were assumed to be 4.291 MTU/cask for intact fuel assemblies and 7.12 MTU/cask for consolidated fuel. The capacities for consolidated fuel included the compacted structural parts resulting from consolidation.

The foregoing capacities were based on the following assumptions:

(a) Two-thirds of the weight of fuel (MTU) would be in the form of PWR fuel, and one-third would be in the form of BWR fuel.

(b) Metal casks would have a capacity for storing 21 intact PWR assemblies or cans of consolidated PWR fuel, or 46 BWR assemblies or cans of consolidated BWR fuel.

(c) Concrete casks would have a capacity for storing 9 intact PWR assemblies or cans of consolidated PWR fuel, or 25 intact BWR assembiies or cans of consolidated BWR fuel.

(d) A PWR fuel assembly would contain $461 \mathrm{kgU}$ and a BWR fuel assembly would contain $183 \mathrm{kgll}$.

(e) A can of consolidated PWR fuel would contain $765 \mathrm{kgu}$ and a can of consolidated BWR fuel would contain $304 \mathrm{kgU}$. This represents an average consolidation ratio of 1.66 , including compacted structural parts.

(3) The costs of the casks for each year were determined by multiplying the number of casks required by the corresponding unit cost of the cask. The unit cost of the casks for any given year was the average unit cost of casks for that year, taking into account the total number of casks produced to that point. Thus by referring to the cumulative numbers of casks required to the mid-point of any year in Tables $\mathrm{C}-1$ or $\mathrm{C}-2$, applying the algorithm described in Appendix $A$ to obtain fabrication costs, and adding the cost of design and licensing (as applicable) and administration and procurement $110 \%$ of fabrication cost), the average cost of casks for the year was obtained. For example, in the case of a SOC in Table C-9, the 
mid-point in the number of casks required for intact fuel in 1994 was 78. Using the algorithm from Appendix $A$, the fabrication cost of the 78th cask produced is calculated to be $\$ 655$-thousand. The total cost of the cask is 10 percent higher, or \$721-thousand, when administration and procurement costs are added. This unit cost for the cask is applied against the total of 23 casks needed that year, giving a total cask cost of $\$ 16,583-$ thousand.

The unit costs for TSCs used to develop the cask costs in the tables did not include any cost for design and licensing, while the unit costs for SOCs and concrete casks did include such costs. The reason for this was that it was assumed that DOE would use TSCS for its rail shipping fleet and that the cost for design and licensing would be incurred by DOE in connection with the procurement of the fleet.

(4) The cost of loading of a storage module and placement thereof in storage was determined by multiplying the sum of the applicable unit costs from Tables B-3 or B-4 by the mathematical product of the number of casks introduced to storage and their respective storage capacities.

(5) The cost of removal of a storage module from storage and preparation for shipment was determined by multiplying the sum of the applicable unit costs from Tables B-3 or B-4 by the mathematical product of the number of casks removed from storage and their respective storage capacities.

(6) In cases involving the use of concrete storage casks, the capital costs of canning and cask loading equipment were included in a separate column in Tables $\mathrm{C}-27$ through $\mathrm{C}-32$. The capital cost of a canning station was estimated in Appendix $B$ (Section 3.1) to be $\$ 145$-thousand, and the capital cost of loading equipment was estimated in Appendix $A$ (Section 3.0 ) to be $\$ 250$-thousand. (The cost of rental equipment was included in the operating costs covered in (4) and (5), above.) It was assumed that this equipment would be added to the utility spent fuel management system as follows:

(a) For cases where the repository commences operation in 1998 -- one set of equipment would be added to the system each year through 1996.

(b) For cases where the repository commences operation in 2003 - - one set of equipment would be added to the system each year through 1996, and two sets of equipment would be added to the system each year during the period 1997 2001 (inclusive).

(c) For cases where the repository commences operation in 2008 - one set of equipment would be added to the system each year through 1996, two sets of equipment would be 
added to the system each year during the period 1997-2001 (inclusive), and three sets of equipment would be added to the system each year during the period 2002-2006 (inclusive).

In cases involving the storage of intact fuel assemblies, each set of equipment costs $\$ 395$-thousand; in cases involving the storage of consolidated fuel, the equipment set costs were only \$250-thousand inasmuch as they did not include the \$145thousand cost of canning equipment -- since it was assumed that the fuel would have already been canned as part of the consolidation process.

(7) The total costs were discounted by two different net discount rates to 1987 ( 3 percent/year and 5 percent/year) to get the total discounted costs (and the total discounted cost for each element thereof) associated with the use of the different casks, in each of the different scenarios involved in terms of 1987 dollars. The reason two different discount rates were used was to illustrate the impact of the discount rate on the unit cost. However, the unit costs resulting from the use of the 3 percent/year discount rate were used for analysis purposes elsewhere in this report.

(8) The unit costs were determined by dividing the total discounted costs by the total discounted amount (MTU) of spent fuel introduced to storage, as follows:

Discounted Costs $=$ Discounted (Unit Costs $\times$ Units of Storage)

Discounted Costs $=$ Unit Costs $\times$ Discounted Units of Storage

Unit cost $=\frac{\text { Discounted Costs }}{\text { Discounted Units of Storage }}$ 
TALE $6-3$

CASE 14 .. CEST OP TSC P

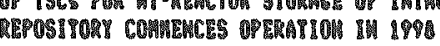

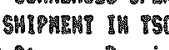

ISOL of

\begin{tabular}{|c|c|c|c|c|c|c|c|c|c|c|c|}
\hline reas & 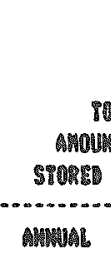 & 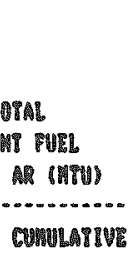 & $\begin{array}{l}\text { M0. Of } \\
\text { METHL } \\
\text { CASESS }\end{array}$ & 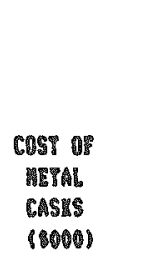 & $\begin{array}{l}\text { COST OP } \\
\text { LOADIAG } \\
\text { P PLACE- } \\
\text { WRET IN } \\
\text { SPORACE } \\
\text { (OOMO) }\end{array}$ & 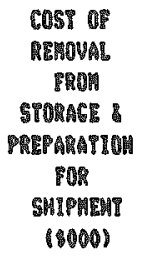 & $\begin{array}{l}\text { TOTAL } \\
\text { (1000) }\end{array}$ & $\begin{array}{c}\text { TOTAL COST } \\
\text { TISC. AT } \\
38 \text { TO } 1907 \\
\text { (6000) }\end{array}$ & $\begin{array}{c}\text { TOTAL COSI } \\
\text { DISG.AP } \\
52 \text { 10 1987 } \\
\text { (1000) }\end{array}$ & 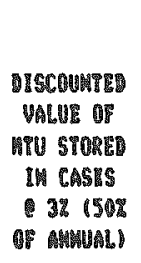 & 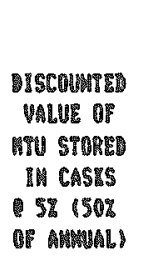 \\
\hline 1987 & 24 & 54 & 1 & 842 & 6 & & 940 & 948 & 940 & 12 & 12 \\
\hline 1980 & 108 & 160 & 4 & 5,472 & 37 & & 5,509 & $\$, 349$ & 5,247 & 51 & 50 \\
\hline 1988 & $16 ?$ & 323 & 9 & 7,916 & 56 & & 7,874 & 7,516 & 7,233 & 77 & 71 \\
\hline 1980 & 12 & 448 & 7 & 6,024 & 43 & & 6,067 & 5,555 & 5,241 & 58 & 54 \\
\hline 1911 & 23 & 687 & 13 & 10,813 & 81 & & 11,064 & 8,830 & 8,102 & 106 & 98 \\
\hline 1902 & 20 & 973 & 15 & 12,454 & 83 & & 12,597 & 10,023 & 9,831 & 123 & 112 \\
\hline 1983 & 25 & 1,231 & 14 & 11,402 & 87 & & 11,569 & 9,600 & 0,633 & 108 & 96 \\
\hline 1894 & 421 & 1,659 & 23 & 11,856 & 143 & & 10,790 & 15,285 & 13,360 & 174 & 152 \\
\hline 1985 & 36 & 2,024 & 20 & 16,020 & 124 & & 16,148 & 12,744 & 10,027 & 184 & 124 \\
\hline 1986 & 45 & 2,178 & 24 & 19,035 & 148 & & 19,184 & 14,703 & 12,346 & 174 & 146 \\
\hline 1907 & 51 & 2,994 & 28 & 22,050 & 174 & & 22,224 & 16,537 & 13,643 & 102 & 158 \\
\hline 1998 & 16 & 3,163 & 9 & 7,037 & 56 & & 7,093 & 3,124 & 4,148 & 81 & 49 \\
\hline 1899 & 27 & 3,435 & 15 & 11,694 & 93 & & 18,787 & 0,267 & 6,563 & 95 & 76 \\
\hline 2000 & 39 & 3,025 & 21 & 16,325 & 130 & & 16,455 & 11,205 & 8,727 & 133 & 103 \\
\hline 2001 & & & (3) & & & 3 & 3 & 2 & 2 & & \\
\hline 2002 & & & $847)$ & & & 52 & 52 & 34 & 25 & & \\
\hline 2003 & & & $(107)$ & & & 118 & 118 & 74 & 54 & & \\
\hline 2004 & & & 1501 & & & 56 & 56 & 31 & 24 & & \\
\hline 2005 & & & & & & & & & & & \\
\hline 2006 & & & & & & & & & & & \\
\hline 2007 & & & & & & & & & & & \\
\hline 2008 & & & & & & & & & & & \\
\hline 2009 & & & & & & & & & & & \\
\hline 2010 & & & & & & & & & & & \\
\hline 2011 & & & & & & & & & & & \\
\hline 2012 & & & & & & & & & & & \\
\hline 2013 & & & & & & & & & & & \\
\hline 2014 & & & & & & & & & & & \\
\hline 2015 & & & & & & & & & & & \\
\hline 2016 & & & & & & & & & & & \\
\hline 2017 & & & & - & & & & & & & \\
\hline 2018 & & & & - & & & & & & & \\
\hline 2019 & & & & & & & & & & & \\
\hline 2020 & & & & & & & & & & & \\
\hline TOTAL & & & 205 & 104,092 & 1,272 & 230 & 167,594 & $1333_{8} 718$ & 116,074 & 1,500 & 1,300 \\
\hline TOTAL 156 :3\% & & & & 132,542 & 1,011 & 143 & & 133,716 & & & \\
\hline UNIT COST/IG & & & & 867.9 & 8.7 & $\$ .1$ & & $\$ 86.7$ & & & \\
\hline POPAL IISC Q5I & & & & 115,093 & 875 & 108 & & & 116,074 & & \\
\hline UMII COST/R6 & & & & 981..1 & 8.8 & 8.1 & & & 180.9 & s & \\
\hline
\end{tabular}




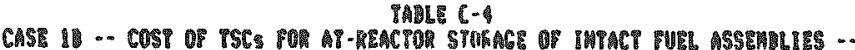

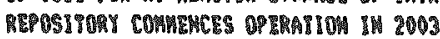
SHIPHEW IN PSC

[50\% of all Stopage Reguirenents Provided by Cashs)

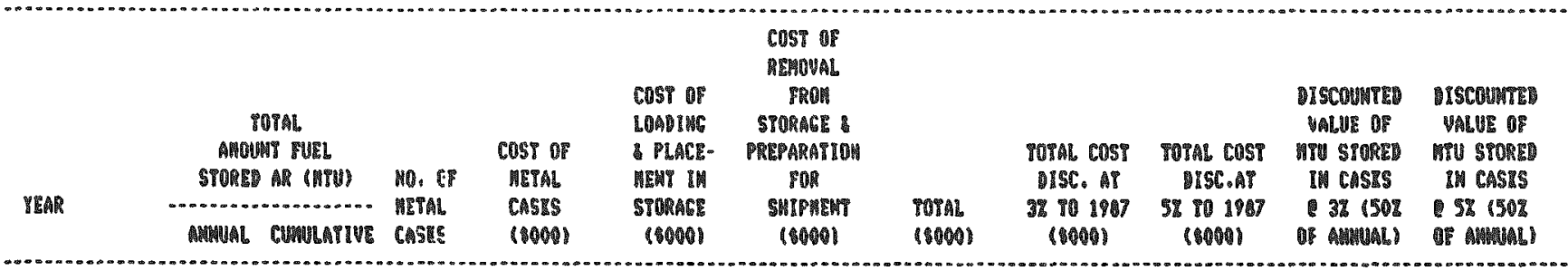

\begin{tabular}{|c|c|c|c|}
\hline 24 & 54 & 1 & 942 \\
\hline 108 & 160 & 1 & 5,472 \\
\hline 163 & 323 & 9 & 9,910 \\
\hline 128 & 449 & 7 & 8,024 \\
\hline 238 & 607 & 13 & 10,983 \\
\hline 288 & 973 & 15 & 12,454 \\
\hline 258 & 1,231 & 14 & 11,482 \\
\hline 428 & 1,659 & 23 & 18,658 \\
\hline 385 & 2,024 & 20 & 18,020 \\
\hline 454 & 2,978 & 24 & 18,035 \\
\hline 516 & 2,994 & 28 & 22,050 \\
\hline 548 & 3,563 & 31 & 24,188 \\
\hline 672 & 4,235 & 36 & $27,8 \mathrm{BA}$ \\
\hline 790 & 5,025 & 43 & 33,040 \\
\hline 944 & 5,869 & 45 & 34,323 \\
\hline 927 & 6,796 & 50 & 37,058 \\
\hline$\$ 15$ & 3,411 & 33 & 24,838 \\
\hline 508 & 7,917 & 26 & 18,510 \\
\hline 619 & 1,536 & 33 & 24,651 \\
\hline \multirow[t]{7}{*}{197} & 0,723 & 10 & 7,459 \\
\hline & & (38) & \\
\hline & & 1921 & \\
\hline & & (54) & \\
\hline & & 1791 & \\
\hline & & $(73)$ & \\
\hline & & (1) & \\
\hline
\end{tabular}

6
37
56
43
93

$948 \quad 948 \quad 12=12$

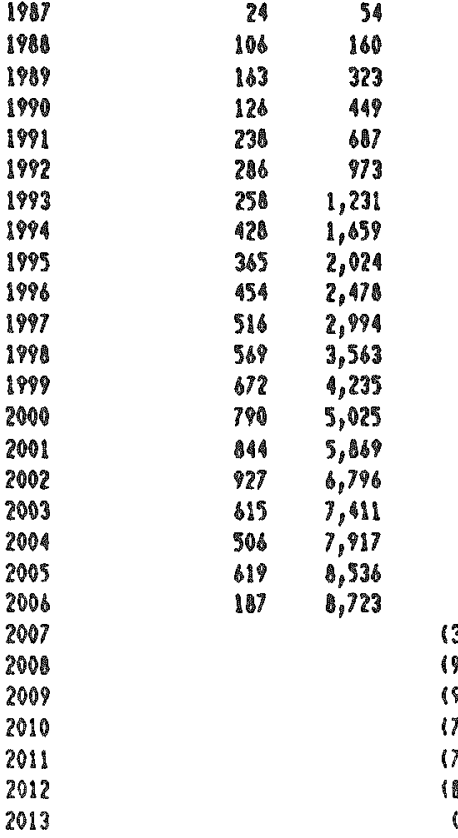

$7,974 \quad 7,518$

$8,067 \quad 5,553$

180840.930

$12,547 \quad 10,823$

11,589

12,744

16,14

$19,104 \quad 14,703$

$24,360 \quad 17,590$

$26,087 \quad 19,700$

ร3. 307

34,602
$38_{1}, 186$

22,680

22,878
24,497

24,497
15,605

15,605
11,801

$\begin{array}{ll}19,671 & 11,901 \\ 24,85 & 18,600\end{array}$

18,600
4,289

\begin{tabular}{|c|c|c|}
\hline 5,247 & 51 & 50 \\
\hline 7,233 & 78 & 74 \\
\hline 5,241 & 58 & 54 \\
\hline 8,102 & 108 & 92 \\
\hline 8,031 & 123 & 112 \\
\hline 0,633 & 100 & $\$ 1$ \\
\hline 13,360 & 174 & 152 \\
\hline 10,927 & 1014 & 124 \\
\hline 12,366 & 174 & 196 \\
\hline 13,643 & 192 & 158 \\
\hline 14,243 & 206 & 160 \\
\hline 15,640 & 236 & 197 \\
\hline 17,663 & 280 & 209 \\
\hline $17,6 / 8$ & 279 & 213 \\
\hline 18,358 & 291 & 223 \\
\hline 11,671 & 192 & 181 \\
\hline 8,583 & 153 & 110 \\
\hline 10,328 & 182 & 120 \\
\hline 2,976 & 58 & 97 \\
\hline
\end{tabular}

467

$364,743-209$

523

360,184

262,991

213,444

3,066

2,493

WhIP COST/ME

260,645

2,058
0.7

268

262,9

$219,607 \quad 1,804 \quad 173$

685.2

TOTAL DISC DS

094.9

213,444 
PALL C-5

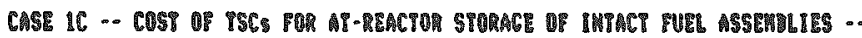

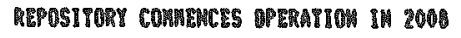

11/ 5 มาขชา

150\% of Ar Stor

\begin{tabular}{|c|c|c|c|c|c|c|c|c|c|c|}
\hline \multirow[b]{3}{*}{ reAn } & \multirow{3}{*}{\multicolumn{2}{|c|}{ STORE⿱ }} & \multirow{3}{*}{$\begin{array}{l}\text { COST OY } \\
\text { METALI } \\
\text { CASIS }\end{array}$} & \multicolumn{3}{|c|}{$\begin{array}{l}\text { COSI OP } \\
\text { REMOVAL }\end{array}$} & \multirow[b]{2}{*}{$\begin{array}{l}\text { TOTAL COST } \\
\text { Disc. Af }\end{array}$} & \multirow{2}{*}{$\begin{array}{c}\text { TOTAL COSP } \\
\text { MISC.APT } \\
\text { 59 } 10 \text { 19A7 }\end{array}$} & \multirow[b]{2}{*}{ 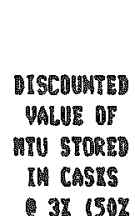 } & \multirow[b]{2}{*}{ 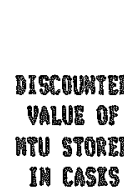 } \\
\hline & & & & $\begin{array}{l}\text { COST OF } \\
\text { LOADING } \\
\text { PLACE- } \\
\text { MEAT IH }\end{array}$ & 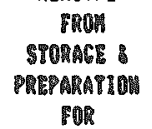 & & & & & \\
\hline & & & & $\begin{array}{c}\text { STORMGE } \\
(1000)\end{array}$ & 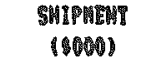 & $\begin{array}{l}\text { TOPAL } \\
\text { (8000) }\end{array}$ & $\begin{array}{c}32701907 \\
180001\end{array}$ & $\begin{array}{c}58901987 \\
(10000)\end{array}$ & 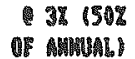 & $\begin{array}{l}5 \% \text { 502 } \\
\text { OF MWUL }\end{array}$ \\
\hline
\end{tabular}

\begin{tabular}{|c|c|c|c|c|c|c|c|c|c|c|c|}
\hline 1987 & 24 & 54 & 1 & 942 & 8 & & 948 & 948 & 998 & 12 & 12 \\
\hline 1988 & 108 & 160 & 6 & 9,472 & 3 & & 5,509 & 5,348 & 5,247 & 51 & 50 \\
\hline 1989 & 163 & 323 & 9 & 8,910 & 56 & & 7,979 & 7,518 & 7,233 & 77 & 74 \\
\hline 1990 & 128 & 448 & 7 & 6,024 & 43 & & 8,087 & 5,553 & 5,241 & 58 & 34 \\
\hline 1991 & 238 & 689 & 13 & 10,913 & 81 & & 11,064 & 8,830 & 8,102 & 100 & 8 \\
\hline 1912 & 208 & 973 & 15 & 12,456 & 93 & & 12,547 & 10,623 & 9,831 & 123 & 112 \\
\hline 1993 & 258 & 1,231 & 14 & 118082 & 87 & & 11,569 & 9,688 & 8,633 & 108 & 96 \\
\hline 1994 & 428 & 1,659 & 23 & 18,658 & 183 & & $16_{8} 79$ & 15,295 & 13,360 & 174 & 852 \\
\hline 1995 & 365 & 2,024 & 20 & 18,020 & 124 & & 16,1144 & 127414 & 10,927 & 198 & 124 \\
\hline 1996 & 4584 & 2,478 & 24 & 18,035 & 149 & & 18,184 & 14,703 & 12,366 & 174 & 146 \\
\hline 1997 & 516 & 2,001 & $2 B$ & 28,050 & 17 解 & & 22,224 & 18,537 & 13,643 & 192 & 150 \\
\hline 1998 & 560 & 3,563 & 31 & 24,186 & 192 & & 24,360 & 17,598 & 14,243 & 206 & 166 \\
\hline 1999 & 672 & 4,235 & 36 & 27,864 & 223 & & 28,007 & 19,700 & 15,640 & 238 & 187 \\
\hline 2000 & 790 & 5,025 & 43 & $33_{8} 240$ & 267 & & 33,307 & $22_{1} 600$ & 17,63 & 269 & 200 \\
\hline 2001 & 84 & 5,869 & 45 & 34,323 & 279 & & 34,602 & 22,878 & 17,478 & 278 & 213 \\
\hline 2002 & 127 & 6,796 & 50 & 37,856 & 310 & & 38,186 & 24,497 & 10,359 & 298 & 283 \\
\hline 2003 & 8,015 & 7,411 & 55 & 11,394 & 341 & & 41,735 & 26,000 & 19,118 & 316 & 232 \\
\hline 2004 & 908 & 8,717 & 48 & 38,603 & 304 & & 36,907 & 22,329 & 16,102 & 274 & 180 \\
\hline 2005 & 1,018 & 9,736 & 55 & 90,899 & 311 & & 41,240 & 24,221 & 17,136 & ลศต & 212 \\
\hline 2006 & 1,007 & 10,823 & 59 & $43_{8} \mathrm{A75}$ & 366 & & 44,041 & 25,116 & 17.428 & 310 & 215 \\
\hline 2007 & 1,003 & 18,900 & 58 & 42,738 & 360 & & 13,009 & 23,663 & 16,244 & 300 & 204 \\
\hline 2008 & 808 & 12,811 & 49 & 35,942 & 304 & & 36,246 & 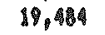 & 13,010 & 244 & 183 \\
\hline 2009 & 350 & 13,644 & 46 & 33,638 & 285 & & 32,923 & 17,704 & 11,507 & 222 & 145 \\
\hline 2010 & 1,195 & 14,799 & 68 & 14. & 378 & & 44,847 & 22,724 & 18,601 & 268 & 185 \\
\hline 2011 & 750 & 15,548 & 41 & $29_{8} 813$ & 254 & & 30,097 & $14: 800$ & 1,332 & 194 & 116 \\
\hline 2012 & & & (22) & & & 24 & 24 & 12 & 7 & & \\
\hline 2013 & & & $(98)$ & & & 109 & 108 & 50 & 31 & & \\
\hline 2014 & & & (103) & & & 114 & 114 & 52 & 31 & & \\
\hline 2015 & & & $(135)$ & & & 150 & 150 & 86 & 38 & & \\
\hline 2016 & & & 11081 & & & 120 & 120 & 91 & 28 & & \\
\hline 2017 & & & (110) & & & 122 & 122 & 50 & 28 & & \\
\hline 2018 & & & $(130)$ & & & 144 & 184 & 58 & 32 & & \\
\hline 2019 & & & $(120)$ & & & 133 & 133 & 52 & 28 & & \\
\hline \multirow[t]{2}{*}{2020} & & & $(1)$ & & & 18 & 16 & 6 & 3 & & \\
\hline & & & 838 & 697,4 & 5,198 & 933 & 643,622 & 412,913 & 314,710 & 4.983 & 3,747 \\
\hline age & & & & 408,275 & 3,312 & 398 & & 412,983 & & & \\
\hline 8 & & & & 318. & 8.7 & 1.1 & & $\$ 93.8$ & & & \\
\hline $25 \%$ & & & & 311,072 & 2,510 & 227 & & & 384,710 & & \\
\hline 8 & & & & 683.3 & 8.7 & .1 & & & $\$ 6400$ & & \\
\hline
\end{tabular}




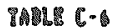

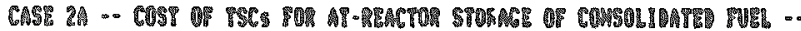

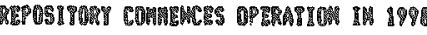
SHIPNEMT IV TSC

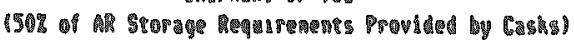

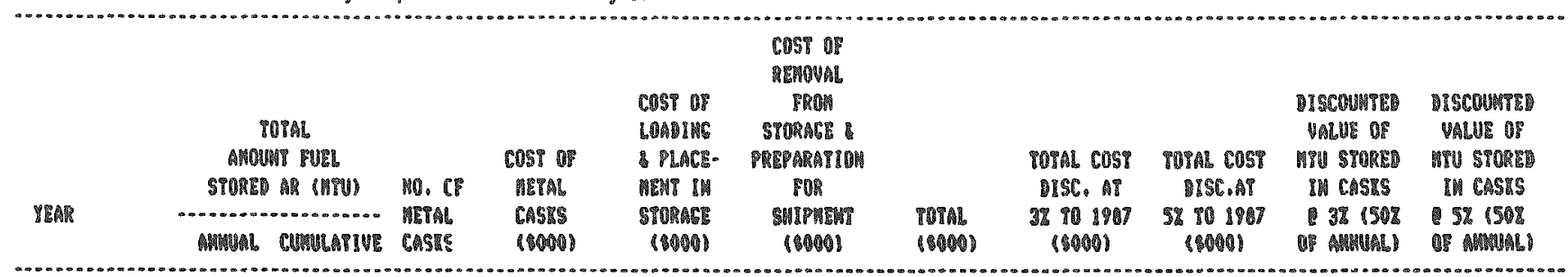

\begin{tabular}{|c|c|c|c|c|c|c|c|c|c|c|c|}
\hline 1907 & 24 & 58 & 1 & 95 & 6 & & 965 & 965 & 965 & 12 & 12 \\
\hline 1981 & 108 & 160 & 4 & 3,718 & 25 & & 3,741 & 3,632 & 3,562 & 51 & 50 \\
\hline 1989 & 183 & 32\% & 5 & 4,500 & 31 & & 4,591 & 4,271 & 4,110 & $n$ & 74 \\
\hline 1990 & 128 & 449 & 4 & 3,520 & 25 & & 3,545 & 3,244 & 3,062 & 50 & 5 \\
\hline 1991 & 233 & 687 & 8 & 6,064 & 43 & & 6,107 & 5,426 & 5,024 & 108 & 98 \\
\hline 1992 & 286 & 973 & 10 & 0,505 & 61 & & 1,360 & 7,390 & 8,712 & 123 & 112 \\
\hline 1999 & 258 & 8,231 & 8 & 6,718 & 49 & & 6,763 & 5,664 & 5,017 & 108 & 96 \\
\hline 1994 & 428 & 1,859 & 14 & 11,007 & 86 & & 11,893 & 9,508 & 0,310 & 174 & 152 \\
\hline 1995 & 385 & 2,021 & 12 & 9,842 & 71 & & 9,918 & 7,820 & 8,711 & 144 & 124 \\
\hline 1986 & 454 & 2,478 & 15 & 12,201 & 92 & & 12,2913 & 9,422 & 7,924 & 174 & 186 \\
\hline 1897 & 518 & 2,894 & 17 & 13,694 & 105 & & 13,799 & 10,287 & 8,471 & 182 & 158 \\
\hline 1996 & 169 & 3,169 & 5 & 4,005 & 38 & & 4,038 & 2,918 & 2,360 & 61 & A \\
\hline 1999 & 272 & 3,435 & 9 & 7,100 & 55 & & 7,244 & 5,081 & 9,034 & 95 & 78 \\
\hline 2000 & 390 & 3,725 & 12 & 9,531 & 74 & & 9,605 & 6.540 & 5,099 & 192 & 103 \\
\hline 2001 & & & (1) & & & 1 & 1 & 1 & 1 & & \\
\hline 2002 & & & 1291 & & & 36 & 36 & 23 & 17 & & \\
\hline 2003 & & & (6) & & & 79 & 78 & A9 & 31 & & \\
\hline 2004 & & & 1301 & & & 37 & 37 & 22 & 16 & & \\
\hline \\
\hline \multirow{2}{*}{\multicolumn{12}{|c|}{$\begin{array}{l}2006 \\
2007\end{array}$}} \\
\hline & & & & & & & & & & & \\
\hline \multicolumn{12}{|l|}{2008} \\
\hline \multicolumn{12}{|l|}{2009} \\
\hline \multirow{2}{*}{\multicolumn{12}{|c|}{$\begin{array}{l}2010 \\
2011\end{array}$}} \\
\hline & & & & & & & & & & & \\
\hline \multicolumn{12}{|l|}{2012} \\
\hline \multirow{2}{*}{\multicolumn{12}{|c|}{2013}} \\
\hline \multirow{2}{*}{\multicolumn{12}{|c|}{$\begin{array}{l}2014 \\
2015\end{array}$}} \\
\hline & & & & & & & & & & & \\
\hline \multicolumn{12}{|l|}{2018} \\
\hline \multirow{2}{*}{\multicolumn{12}{|c|}{$\begin{array}{l}2017 \\
2011\end{array}$}} \\
\hline & & & & & & & & & & & \\
\hline \multicolumn{12}{|l|}{2019} \\
\hline \multicolumn{12}{|l|}{2020} \\
\hline & & & 123 & 102,047 & 756 & 152 & $102_{3} 958$ & 32,247 & 71,456 & 1,500 & 1,308 \\
\hline $3 \%$ & & & & 81,550 & 102 & 85 & & 2,297 & & & \\
\hline & & & & 894.1 & 8.4 & 8.1 & & 854.5 & & & \\
\hline & & & & 70,085 & 522 & 10 & & & 11,456 & & \\
\hline & & & & 854.3 & 8.4 & 0.1 & & & 54.7 & & \\
\hline
\end{tabular}




\begin{tabular}{|c|c|c|c|c|c|c|c|c|c|}
\hline & & 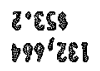 & & & SII & $200^{\circ} 1$ & $\begin{array}{l}0.258 \\
45^{8} \operatorname{lic}\end{array}$ & & 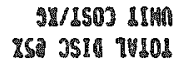 \\
\hline & & & $\begin{array}{l}0_{0} \mathrm{ESE} \\
\operatorname{mes} \mathrm{EQ1}\end{array}$ & & 801 & $\cos 2$ & $\begin{array}{l}5258 \\
811291\end{array}$ & & 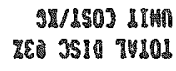 \\
\hline $\cos ^{\circ} 2$ & $900^{\circ} \varepsilon$ & แ9ด' 28 & "xes & $D^{\prime \prime} I^{\circ} \triangle E 2$ & 6ne & $96 L^{d} \mathbb{1}$ & $\triangle 20^{6} L E 2$ & 192 & TV10d \\
\hline
\end{tabular}

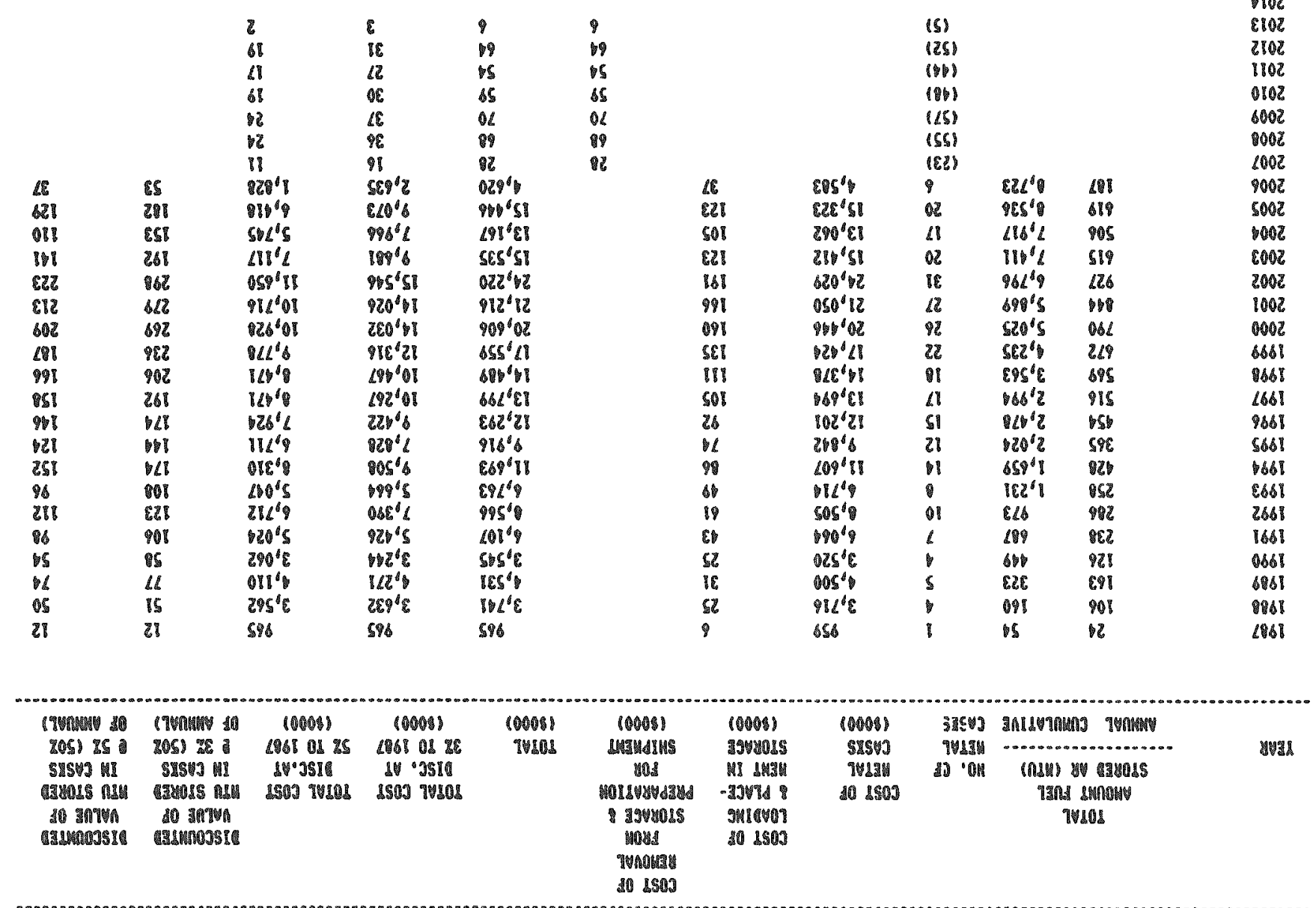

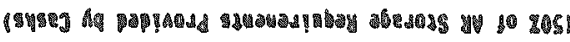

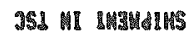

cองว

20185 118

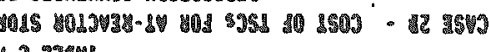


IAME $[-3$

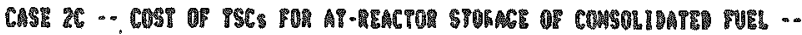

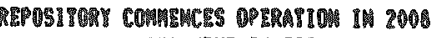

$11 / 3 / 1 \%$

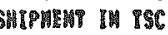

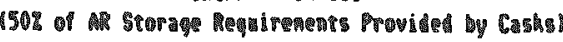

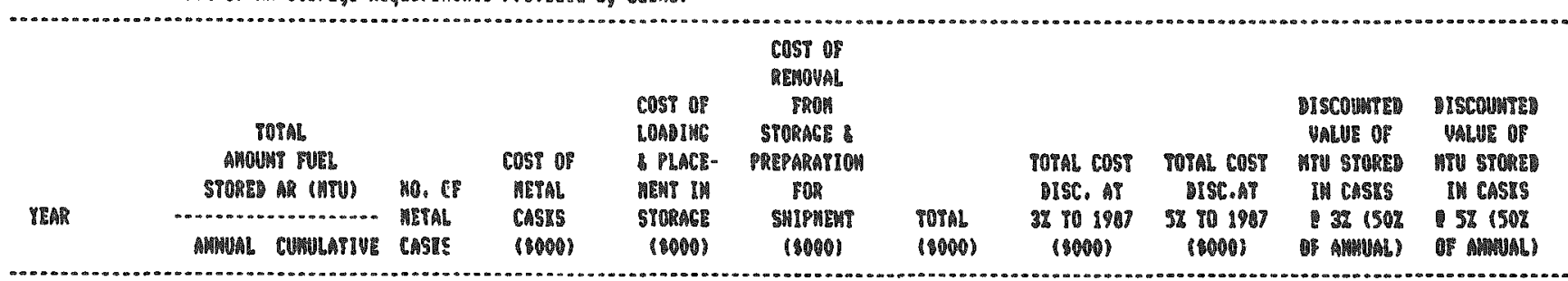

1997
1988
1989
1990
1901
1992
1993
1994
1999
1998
1997
1998
1919
2000
2001
2002
2003
2001
2005
2008
2007
2000
2009
2010
2011
2012
2013
2014
2015
2016
2017
2018
2019
2020

TOTAL

\section{TOTAL DISC อง}

WIII COST/RS

TOYML DISC อ5ร

MIII COST/AG

\begin{tabular}{|c|c|c|c|c|c|c|c|c|c|c|}
\hline 24 & 54 & 1 & 959 & 8 & & 985 & 965 & 985 & 12 & 12 \\
\hline 106 & 160 & 4 & 3,716 & 25 & & 3,741 & 3,632 & 3,562 & 51 & 50 \\
\hline 183 & 323 & 5 & 4,500 & 31 & & 4,531 & 8,271 & 1110 & 77 & 74 \\
\hline 126 & 449 & 4 & 3,520 & 25 & & 3,545 & 3,244 & 3,062 & 58 & 54 \\
\hline 236 & 687 & 7 & SpOBA & 43 & & 8,107 & 5,426 & 5,024 & 108 & 98 \\
\hline $2 A B$ & 973 & 10 & 8,505 & $d 1$ & & 8.586 & 7,390 & 6,712 & 123 & 112 \\
\hline 258 & 1,231 & 1 & 6,710 & 19 & & 6,783 & 5,864 & 5,047 & 108 & 96 \\
\hline 428 & 1,659 & 14 & 11,607 & 86 & & 11,693 & 9,500 & 1,310 & 174 & 152 \\
\hline 385 & 2,024 & 12 & 9,842 & 74 & & 8,916 & 7,828 & 6,711 & 194 & 124 \\
\hline 950 & 2,478 & 15 & 12,201 & 92 & & 12,283 & 9,122 & 7,224 & 174 & 196 \\
\hline 516 & 2,9 & 17 & 13,604 & 105 & & 13,799 & 10,267 & 8,871 & 192 & 158 \\
\hline 549 & 3,563 & 10 & 14,370 & 111 & & 14,468 & $10_{8}, 467$ & 8,471 & 208 & 166 \\
\hline 872 & 0,235 & 22 & 17,426 & 135 & & 17,559 & 12,316 & 8,778 & 238 & 197 \\
\hline 790 & 5,025 & 28 & 20,446 & 160 & & 20,806 & 14,032 & 10,928 & 269 & 200 \\
\hline 844 & 5,868 & 27 & 21,050 & 186 & & 21,216 & 11,028 & 10,716 & 278 & 213 \\
\hline 927 & 8,798 & 31 & 24,029 & 191 & & 24,220 & 15,548 & 21,650 & 290 & 223 \\
\hline 1,015 & 7,811 & 32 & 24,820 & 197 & & 24,821 & 15,487 & 11,37 & 318 & 232 \\
\hline 906 & 8,717 & 30 & 22,950 & 184 & & 23,134 & 13,997 & 10,093 & 274 & 198 \\
\hline 1,019 & 8736 & 33 & 25,134 & 203 & & $25,3,37$ & 14,883 & 10,528 & 299 & 212 \\
\hline 1,097 & 10,123 & 35 & 26,499 & 215 & & 26,714 & 15,235 & 10,572 & 310 & 215 \\
\hline 1,003 & $11,90 \%$ & 35 & 26,381 & 215 & & 26,596 & 10,720 & 10,024 & 300 & 204 \\
\hline 900 & 12,814 & 29 & 21,794 & 178 & & 21,972 & 11,812 & 7,807 & 244 & 163 \\
\hline 850 & 13,664 & 28 & 20,979 & 172 & & 21,151 & 11,039 & 7,231 & 222 & 145 \\
\hline 1,135 & 14,799 & 37 & 27,597 & 227 & & 27,824 & 14008 & 8,058 & 288 & 185 \\
\hline \multirow[t]{15}{*}{750} & 15,549 & 28 & 17,847 & 180 & & 17,995 & 8,952 & 5,560 & 184 & 118 \\
\hline & & (13) & & & 16 & 16 & 4 & 5 & & \\
\hline & & (991) & & & 73 & 73 & 34 & 20 & & \\
\hline & & 1621 & & & 76 & 76 & 34 & 20 & & \\
\hline & & (26) & & & 100 & 100 & 94 & 25 & & \\
\hline & & 1651 & & & 80 & 10 & 34 & 18 & & \\
\hline & & $(69)$ & & & 22 & 82 & 34 & 19 & & \\
\hline & & 1781 & & & 96 & 96 & 38 & 21 & & \\
\hline & & 1721 & & & 4 & 89 & 34 & 19 & & \\
\hline & & 89 & & & 11 & 11 & 4 & 2 & & \\
\hline & & 504 & 392,454 & 3,009 & 622 & 396,175 & 256,373 & 193,930 & 4,843 & 3,798 \\
\hline & & & 252,134 & 18975 & 268 & & 254,373 & & & \\
\hline & & & 351.0 & 8.4 & 8.1 & & 05105 & & & \\
\hline & & & 192,207 & 1,890 & 151 & & & 103,938 & & \\
\hline & & & $\$ 51.3$ & 8.8 & 8.0 & & & 53 & & \\
\hline
\end{tabular}


ThBLE C-10

CASE 38 -. COST OF SOCS POR AP-BEACTOR STORAGE OP IMTACT PUEL ASSEMRLIES -

REPOSITOAY COWEEMCES OPERATIOM IN 2003

11/ 911987

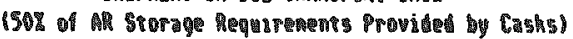

\begin{tabular}{|c|c|c|c|c|c|c|c|c|c|c|}
\hline \multirow[t]{2}{*}{$Y E^{2}$} & 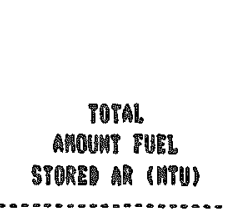 & \multirow{2}{*}{$\begin{array}{l}\text { MO. Of } \\
\text { MEIAL } \\
\text { CASES }\end{array}$} & \multirow{2}{*}{$\begin{array}{l}\text { COST OP } \\
\text { HETAL } \\
\text { CASES } \\
\text { 10000) }\end{array}$} & \multirow[t]{2}{*}{ 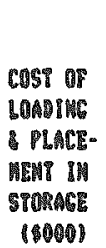 } & \multirow[t]{2}{*}{ 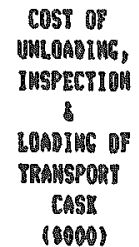 } & \multirow{2}{*}{$\begin{array}{l}\text { TOTAL } \\
\text { (10000) }\end{array}$} & \multirow{2}{*}{ 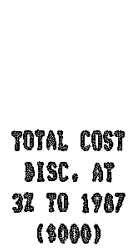 } & \multirow{2}{*}{ 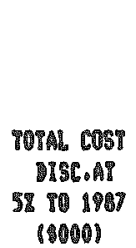 } & \multirow[t]{2}{*}{ 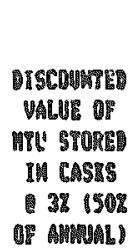 } & \multirow[t]{2}{*}{ 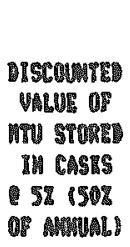 } \\
\hline & ANUบดL CEพULAPIUE & & & & & & & & & \\
\hline
\end{tabular}

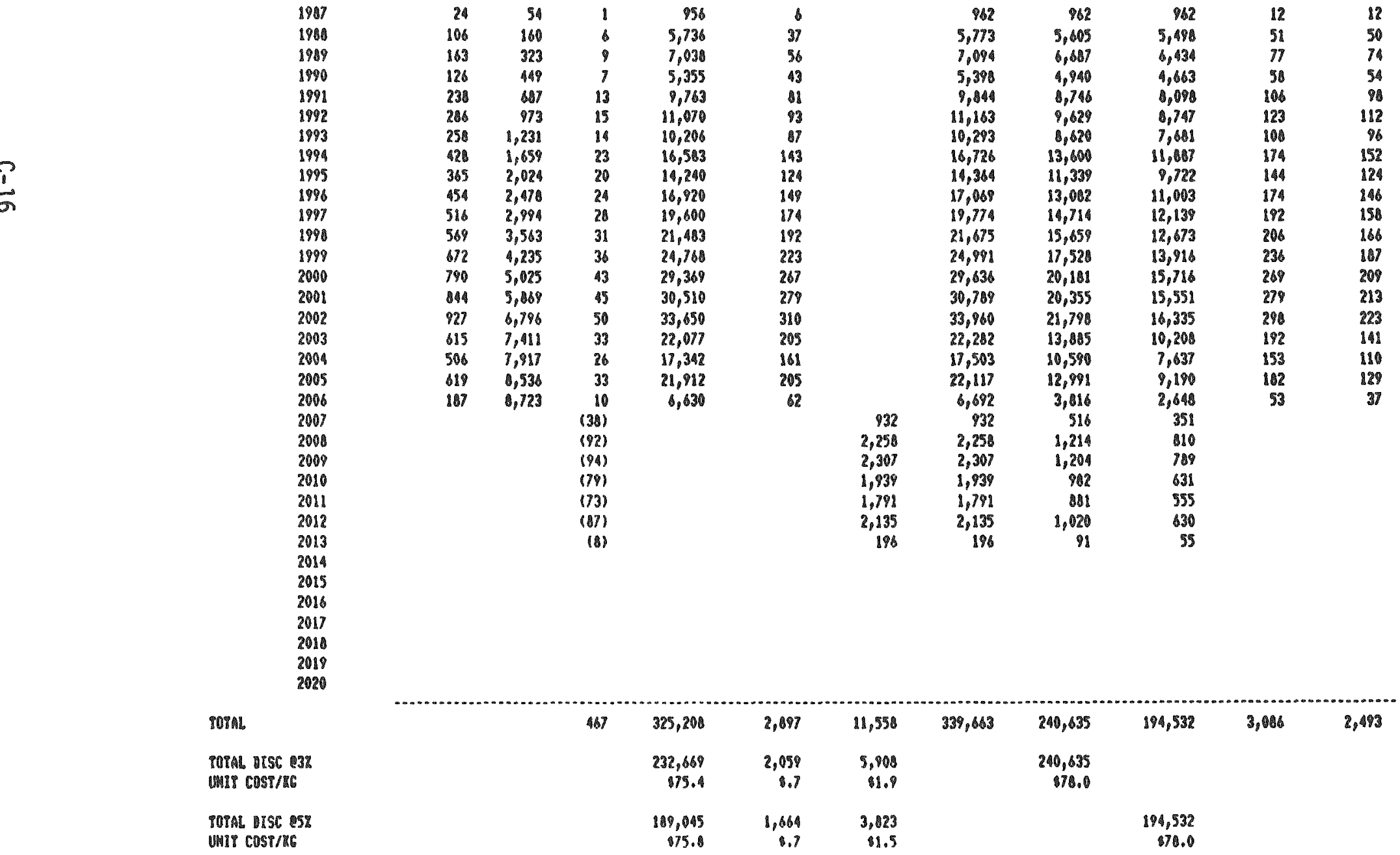


TAMLE C- II

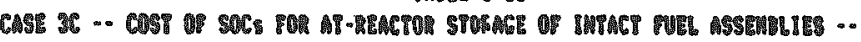

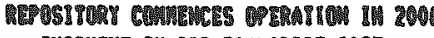

ate

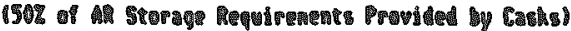

\begin{tabular}{|c|c|c|c|c|c|c|c|c|c|c|}
\hline צ思骨 & 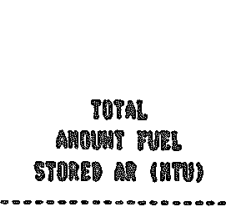 & $\begin{array}{l}\text { HO. OP } \\
\text { METAL }\end{array}$ & $\begin{array}{l}\text { COST OI } \\
\text { METAL } \\
\text { CASMS }\end{array}$ & 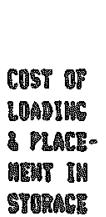 & 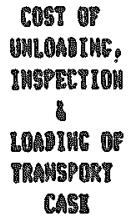 & & 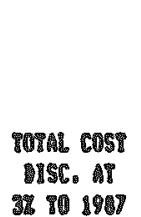 & $\begin{array}{c}\text { roput cost } \\
\text { ISC.AP } \\
52 \text { ro } 1987\end{array}$ & 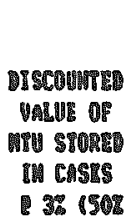 & 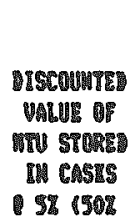 \\
\hline & 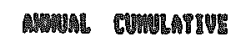 & CAsme & 160001 & 80001 & $(10000)$ & 1 & 10001 & $(0000)$ & 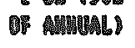 & 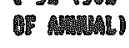 \\
\hline
\end{tabular}

\begin{tabular}{|c|c|c|c|c|c|c|c|c|c|c|c|}
\hline 1987 & 24 & 54 & 1 & $85 \%$ & 8 & & 982 & 982 & 962 & 12 & 12 \\
\hline 1988 & 108 & 860 & 6 & 5,736 & 37 & & $\$, 773$ & 5,605 & 5,498 & 58 & 30 \\
\hline 1989 & 183 & 323 & 9 & 7,030 & 58 & & 7,096 & $6,9 M 7$ & 8,438 & 77 & 74 \\
\hline 1890 & 126 & 448 & 8 & 5,9355 & 43 & & 5,396 & 4,940 & 4.683 & 50 & 54 \\
\hline 1991 & 238 & 687 & 13 & 9,783 & 81 & & 9884 & 8,748 & 8000 & 108 & 98 \\
\hline 1892 & 285 & 973 & 15 & 11,070 & 93 & & 18,163 & 8,628 & 8,747 & 123 & 112 \\
\hline 1893 & 258 & 1,231 & 11 & 10,206 & 87 & & 10.283 & 8,620 & 8,681 & 108 & 16 \\
\hline 1994 & A28 & 1,698 & 23 & 16.5 & 143 & & 16,728 & $13_{8} 600$ & $11, \frac{9}{4} 7$ & 174 & 152 \\
\hline 1995 & 365 & 2,024 & 20 & 14,240 & 124 & & 14,384 & 11,339 & 8,722 & 144 & 124 \\
\hline 1896 & 854 & 2,478 & 24 & 160920 & 189 & & 17,008 & $13_{3}, 0012$ & 11,0008 & $17 \mathrm{~A}$ & 146 \\
\hline 1997 & 516 & 2,0 & 2 & 19,600 & 178 & & 18,774 & 18,714 & 12,139 & 192 & 158 \\
\hline 199 & 588 & 3,563 & 31 & 21,083 & 192 & & 21,675 & 15,658 & 12,678 & 200 & 16 \\
\hline 199 & bा2 & ,, 235 & 30 & $26,7 \mathrm{~m}$ & 223 & & 26,591 & 17,528 & 13 , 118 & 236 & 197 \\
\hline 2000 & 780 & 5,025 & 48 & 87,367 & 287 & & 27,636 & 20,161 & 15,710 & 200 & 200 \\
\hline 2001 & 644 & 5,468 & 45 & 30,5180 & 278 & & 30,789 & 20,355 & 15,551 & 278 & 213 \\
\hline 2002 & 927 & 8,798 & 50 & 33,550 & 310 & & 33,960 & 21,790 & 16,335 & 290 & 223 \\
\hline 2003 & 1,015 & 7,811 & 55 & 36,795 & 341 & & 37,136 & 23,142 & 87,013 & 316 & $23 \%$ \\
\hline 2004 & 906 & 8,717 & 4 & 32,536 & 304 & & $32_{8} 640$ & 19,068 & 14,328 & 274 & $18 \%$ \\
\hline 2005 & 1,018 & 8,736 & 55 & 36,355 & 341 & & 36,698 & 21,555 & 15,248 & 299 & 212 \\
\hline 2006 & 1,007 & 10,823 & 59 & 38,822 & 384 & & 39,100 & 22,346 & 15,508 & 310 & 215 \\
\hline 2007 & 1,083 & 11,906 & 58 & 37,990 & 360 & & 38,350 & 21,233 & 14,454 & 300 & 204 \\
\hline 2008 & 900 & 12,014 & 49 & 31,946 & 304 & & 32,252 & 17,237 & 11,577 & 244 & 163 \\
\hline 2009 & 850 & 13,664 & 4 & 29,900 & 285 & & 30,865 & 15,758 & 10,310 & 222 & 145 \\
\hline 2010 & 18,135 & 14,799 & 61 & 39,528 & 378 & & 39,906 & 20,220 & 12,992 & 288 & 185 \\
\hline 2011 & 750 & 15,548 & 41 & 26,527 & 254 & & 26,781 & $13_{8} 175$ & 8,390 & 104 & 116 \\
\hline 2012 & & & $(22)$ & & & 500 & 540 & 258 & 158 & & \\
\hline 2013 & & & 1901 & & & 2,405 & 2,405 & 1,115 & 676 & & \\
\hline 2014 & & & 11031 & & & 2,528 & 2,528 & 1,138 & 677 & & \\
\hline 2015 & & & (135) & & & 3,313 & 3,313 & 1,448 & 315 & & \\
\hline 2018 & & & 11001 & & & 2,650 & 2,650 & 1,125 & 644 & & \\
\hline 2017 & & & 111801 & & & 2,699 & 2,699 & 1,112 & 625 & & \\
\hline 2010 & & & $(130)$ & & & 3,190 & 3,100 & 1,278 & 703 & & \\
\hline 2019 & & & $(120)$ & & & 2,845 & 2,945 & 1,148 & 618 & & \\
\hline \multirow[t]{2}{*}{2020} & & & (18) & & & 364 & 344 & 130 & 60 & & \\
\hline & & & 33 & 567,646 & $\$, 198$ & 20,813 & 593,460 & 378,822 & 205,795 & 4948 & 3,747 \\
\hline 39 & & & & 384,786 & 3,312 & 0,794 & & 376,822 & & & \\
\hline & & & & 775.8 & 8.7 & 11.0 & & 176.2 & & & \\
\hline $5 \%$ & & & & 278,258 & 2,510 & 5,016 & & & 205,785 & & \\
\hline & & & & 174.3 & 0.7 & 11.3 & & & 676.3 & & \\
\hline
\end{tabular}


PASLE C-12

CASE

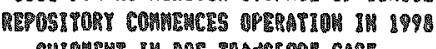

(502 of a stopege Requrenents Provitued by Casts)

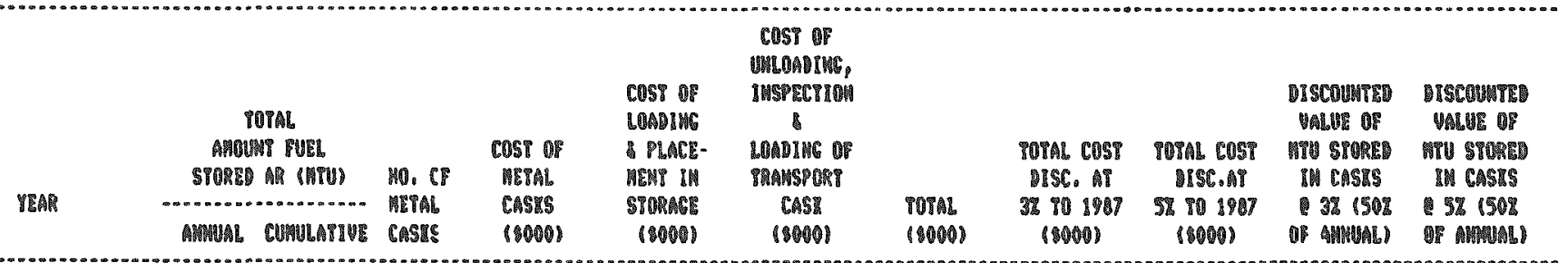

\begin{tabular}{|c|c|c|c|c|c|c|c|c|c|c|c|}
\hline 1987 & 21 & 54 & 1 & 856 & $\$$ & & $\$ 82$ & 982 & 962 & 12 & 12 \\
\hline 1988 & 106 & 100 & 4 & 3,824 & 25 & & $3_{p}, 6 \%$ & 3,738 & 3,065 & 51 & 50 \\
\hline 1989 & 163 & 323 & 5 & 9,780 & 31 & & 8) & 4,535 & 4,363 & $n$ & 74 \\
\hline 1990 & 126 & 449 & 4 & 3,120 & 25 & & 3,453 & 2,885 & 2,723 & 51 & \$4 \\
\hline 1981 & 238 & 887 & 7 & 5,390 & 43 & & 5,433 & 4,028 & 4,870 & 106 & 98 \\
\hline 1982 & 296 & 173 & 10 & 7,560 & b1 & & 7,621 & 6,574 & 5,072 & 123 & 112 \\
\hline 1993 & 250 & 1,231 & 1 & 5,860 & 9 & & 8,017 & 5,039 & 4890 & 100 & 9 \\
\hline 1994 & 428 & 1,659 & I4 & 10,310 & $a$ & & 10,404 & 8859 & 7,391 & 174 & 152 \\
\hline 1995 & 365 & 2,028 & 12 & 18798 & 74 & & 8,822 & 8,984 & 5,971 & 144 & 124 \\
\hline 1996 & 454 & 2,470 & 15 & 10,345 & 82 & & 10,937 & 8,382 & 7,050 & 174 & 196 \\
\hline 1997 & 516 & 2,894 & 17 & 12,172 & 105 & & 12,277 & 9,135 & 7,5997 & 192 & 158 \\
\hline 1896 & 169 & $3_{8} 163$ & $g$ & 3.560 & 31 & & 3,591 & 2,594 & 2,098 & 61 & 49 \\
\hline $19 P 9$ & 272 & 3,435 & 8 & $\$ 390$ & 53 & & 6,895 & 1,521 & $3,5 \mathrm{Ag}$ & 95 & 78 \\
\hline 2000 & 390 & 3,023 & 12 & 0,872 & 74 & & 8,596 & 3,018 & 4532 & 123 & 103 \\
\hline 2001 & & & (I) & & & 18 & 17 & 11 & 9 & & \\
\hline 2002 & & & 1291 & & & $\$ 90$ & 980 & 315 & 236 & & \\
\hline 2003 & & & $(84)$ & & & 1,062 & 1,082 & $\$ 74$ & 496 & & \\
\hline $200 \mathrm{~A}$ & & & 1301 & & & 507 & 507 & 307 & 221 & & \\
\hline 2005 & & & & & & & & & & & \\
\hline 2006 & & & & & & & & & & & \\
\hline 2007 & & & & & & & & & & & \\
\hline 2000 & & & & & & & & & & & \\
\hline 2009 & & & & & & & & & & & \\
\hline 2010 & & & & & & & & & & & \\
\hline 2014 & & & & & & & & & & & \\
\hline 2012 & & & & & & & & & & & \\
\hline 2013 & & & & & & & & & & & \\
\hline 2014 & & & & & & & & & & & \\
\hline 2015 & & & & & & & & & & & \\
\hline 2016 & & & & & & & & & & & \\
\hline 2017 & & & & & & & & & & & \\
\hline 2010 & & & & & & & & & & & \\
\hline 2018 & & & & & & & & & & & \\
\hline 2020 & & & & & & & & & & & \\
\hline TOTAL & & & 123 & 92,111 & 756 & 2,098 & 94,854 & 75,741 & 65,778 & 1,500 & 1,328 \\
\hline TOPAL MISE $13 \%$ & & & & 73,332 & 302 & 1,307 & & 75,791 & & & \\
\hline แมก cosirge & & & & 149.0 & 1.4 & 8.9 & & 50.2 & & & \\
\hline POPML MISG OTS & & & & $\$ 4,298$ & 522 & 961 & & & 85,779 & & \\
\hline WMIS COST/RC & & & & 948.2 & 0.4 & 8.7 & & & 550.4 & & \\
\hline
\end{tabular}




\begin{tabular}{|c|c|c|c|c|c|c|c|c|c|}
\hline & & $\begin{array}{l}\text { Sogen } \\
920028\end{array}$ & & & $80 e^{\circ}$ & 2008 & 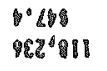 & & 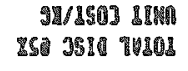 \\
\hline & & & 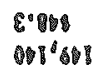 & & 10.8 & 6E2 & pocos & & 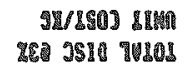 \\
\hline 8 & 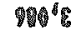 & 920002 & Ob! $I^{8} \operatorname{sit}$ & $1950^{\circ} 802$ & $200^{6} 8$ & $9 L^{8} !$ & $90 e^{6} \mathrm{EOL}$ & $m 2$ & ขอมอ \\
\hline
\end{tabular}

\begin{tabular}{|c|c|c|c|c|c|c|c|c|c|c|c|}
\hline & & 12 & 6 & 50 & $\mathrm{St}$ & & & (\$) & & & $\begin{array}{l}102 \\
8102\end{array}$ \\
\hline & & ดथ & 020 & 810 & 610 & & & (25) & & & 2102 \\
\hline & & IEZ & 92 & 62 & 002 & & & $(9)$ & & & 1102 \\
\hline & & 02 & IIV & 218 & III & & & $(96)$ & & & 910 \\
\hline & & $\$ 276$ & $\cos$ & 698 & 626 & & & (us) & & & 6002 \\
\hline & & UCE & 005 & $0 \approx 6$ & DEs & & & 1558 & & & 8002 \\
\hline & & All & $\$ 2$ & 8ถe & $\Delta A$ & & & (EZ) & & & 2002 \\
\hline $\mathscr{I} \varepsilon$ & 85 & 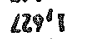 & tot & III" & & LC & $1010^{6} 1$ & 9 & $22 d^{6}$ & 691 & 9002 \\
\hline 28 & 29: & OUL & $\& \angle 0^{\circ}$ & $\varepsilon L^{\prime} \varepsilon$ & & 86 & $029^{8} \mathrm{CI}$ & $\Delta Z$ & $9 \mathrm{ged}^{\prime}$ & Bif & 5002 \\
\hline OII & ESI & III & $090^{\circ} \mathrm{l}$ & $P L^{8} U$ & & $\operatorname{sel}$ & II) & 41 & $186^{4} 6$ & 905 & 6002 \\
\hline 10 & 281 & $\operatorname{ztc}^{6}$ & $519^{6}$ & Eखी है। & & 828 & Q & Q & I10' & $\$ 10$ & 8002 \\
\hline 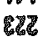 & TSE & $998^{\circ} 0$ & EEO & oss.12 & & 167 & ASE 18 & IE & $988^{6} 9$ & (2) & 2002 \\
\hline ธะเ & 418 & 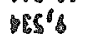 & 00621 & $110^{8} 91$ & & 991 & $114^{6} 91$ & Le & $68^{\circ} \mathrm{g}$ & me & 1002 \\
\hline 602 & 898 & $828^{8}$ & 106 & $-E^{6}$ & & 908 & $47^{3} 9$ & 4 & $520^{\circ} \mathrm{s}$ & 061 & 000 \\
\hline IVI & 962 & $002^{\prime}$ & ose or & $229^{\circ} 5$ & & SEl & 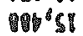 & 22 & $58 a^{6}$ & 218 & S6S1 \\
\hline 898 & 902 & $185^{\circ} l$ & $278^{6} 6$ & $160^{\prime} 21$ & & กเ & $92 L^{6} 21$ & 81 & $\cos ^{d} \mathrm{C}$ & 698 & OSB \\
\hline จS & E6b & $\operatorname{ces}^{16} 6$ & SEIO & $\angle Z^{6} 21$ & & sol & $24 I^{\prime} 21$ & LI & $168^{6} 2$ & PIS & LSOI \\
\hline 898 & 148 & $050^{\circ} \mathrm{s}$ & $20 \varepsilon^{6}$ & LES & & 26 & $539^{8} 01$ & si & 0404 & 15 & 2851 \\
\hline 621 & 01 & $126^{\circ} \mathrm{S}$ & $94^{6}$ & 22010 & & 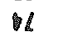 & $L^{\prime}$ & 21 & 12012 & 598 & SSESI \\
\hline 258 & 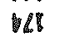 & $\omega E^{\prime} L$ & 6556 & Dos & & 9 & vicior & 1 & $60^{\circ} 8$ & 2 & DSs? \\
\hline 96 & 808 & $00^{\circ}$ & $\mathrm{SeO}^{6} \mathrm{~s}$ & $210^{\circ}$ & & 80 & $906^{6} \mathrm{~s}$ & 0 & $12^{\prime} 1$ & 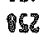 & CSAI \\
\hline EII & EZ & $2 \operatorname{Ls}^{8} 5$ & $\angle S^{d}$ & 1291 & & 5 & $095^{8} 1$ & 01 & ess & 902 & 28S5 \\
\hline 98 & 901 & 040 & L29' & cenos & & 80 & ose's & 1 & 209 & จ⿰纟己 & 1861 \\
\hline \$s & 85 & $\operatorname{cec} L^{6}$ & 5986 & $\mathrm{CSI}^{\mathrm{C}}$ & & $\mathrm{S}$ & $92 I^{18}$ & 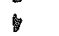 & $\$$ & 9ell & 0681 \\
\hline 2 & $u$ & $\cos ^{6}$ & $\operatorname{scs}^{6}$ & 1186 & & IE & 0 & 5 & 228 & (91 & 6861 \\
\hline 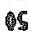 & 15 & $590^{\prime}$ & $x L^{\prime} \bar{C}$ & S60 & & se & $220^{\circ}$ & 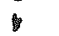 & 901 & 901 & 9865 \\
\hline 21 & 21 & 298 & 298 & 296 & & 9 & 898 & 1 & 65 & $z^{\circ}$ & Les? \\
\hline
\end{tabular}

\begin{tabular}{|c|c|c|c|c|c|c|c|c|c|c|}
\hline 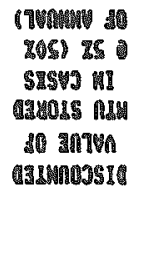 & 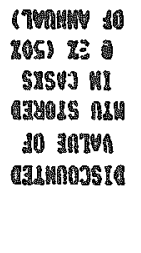 & 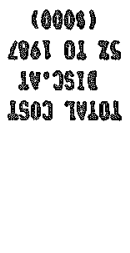 & 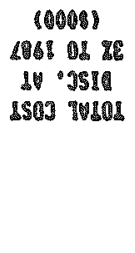 & $\begin{array}{c}\text { 1000ง) } \\
\text { TV108 }\end{array}$ & 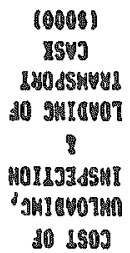 & 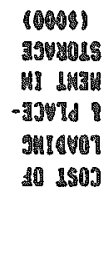 & 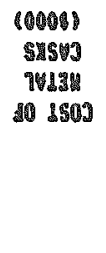 & 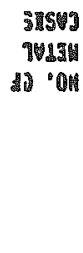 & 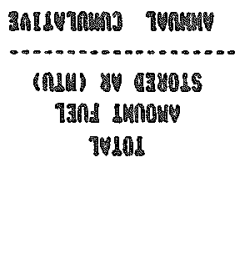 & 84ต \\
\hline
\end{tabular}

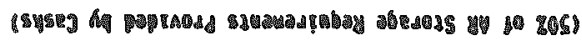

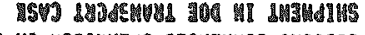


TALIE $C-14$

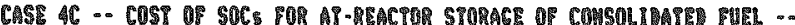

REPESITEQY CONTERCES OPERATIOM IN 2000

MHPWEAY IS BOP TRAMSPOAT CASE

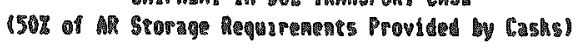

\begin{tabular}{|c|c|c|c|c|c|c|c|c|c|c|}
\hline \multirow[b]{3}{*}{ PRAR } & \multirow{3}{*}{\multicolumn{2}{|c|}{ 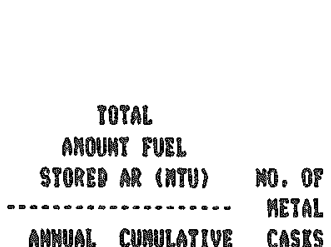 }} & \multirow{3}{*}{$\begin{array}{l}\text { CMST OP } \\
\text { METAL } \\
\text { CASES } \\
\text { (OOOOO) }\end{array}$} & \multicolumn{3}{|c|}{$\begin{array}{c}\text { cost of } \\
\text { UMLAลB! }\end{array}$} & \multirow{3}{*}{$\begin{array}{c}\text { TOTAL, COSI } \\
\text { ISC. AT } \\
\text { 3\% TO } 1897\end{array}$} & \multirow{3}{*}{ 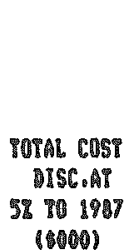 } & \multirow{3}{*}{ 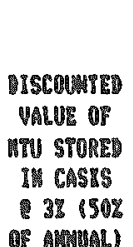 } & \multirow{3}{*}{ 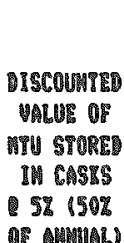 } \\
\hline & & & & $\begin{array}{l}\text { PLACE- } \\
\text { WEM II }\end{array}$ & $\begin{array}{l}\text { LOABIME OE } \\
\text { PRAMSPORT }\end{array}$ & & & & & \\
\hline & & & & $\begin{array}{l}\text { STORACE } \\
(5000)\end{array}$ & $\begin{array}{c}\text { CASE } \\
\text { (6000) }\end{array}$ & $\begin{array}{l}\text { TOYAL } \\
(8000)\end{array}$ & & & & \\
\hline
\end{tabular}

\begin{tabular}{|c|c|c|c|c|c|c|c|c|c|c|}
\hline 24 & 54 & I & 956 & 6 & & 862 & 982 & 962 & 12 & 12 \\
\hline 100 & 180 & 4 & 3,324 & 25 & & 3,848 & 3,736 & 3,645 & 51 & 50 \\
\hline 163 & 323 & $g$ & 9,780 & 31 & & 9011 & 4,535 & 1,363 & $\pi 7$ & 74 \\
\hline 126 & 449 & 1 & 3,120 & 25 & & 3,153 & 2,8 805 & 2,723 & 50 & 54 \\
\hline 238 & 687 & 7 & 5,390 & 4 & & 5,433 & 4,027 & 8,470 & 106 & 98 \\
\hline 286 & 973 & 10 & 7,560 & B! & & 78621 & 6,574 & 5,972 & 123 & 112 \\
\hline 258 & 1,231 & 8 & 5,960 & 48 & & 6,017 & 5,039 & 9,490 & $10 \mathrm{H}$ & 86 \\
\hline 428 & 1,659 & 14 & 10,310 & 16 & & 10,400 & 8,459 & 7,394 & 174 & 152 \\
\hline 365 & 2,024 & 12 & 0,740 & 74 & & 8,822 & 8,964 & 5,971 & 144 & 121 \\
\hline 854 & 2,478 & 15 & 10,845 & 92 & & 10,937 & 8,382 & 8,050 & 174 & 146 \\
\hline 516 & 2,984 & 17 & 12,172 & 105 & & 12,277 & 8,135 & 7,537 & 192 & 150 \\
\hline 569 & 3,563 & 18 & 12,7100 & III & & 12,898 & 8,312 & 7,537 & 206 & 160 \\
\hline 872 & 1,235 & 22 & 15,100 & 135 & & 15,823 & 10,956 & 8,700 & 236 & 187 \\
\hline 790 & 5,025 & 28 & 18,174 & 160 & & 10,334 & $12,48 \mathrm{~A}$ & 8,723 & 269 & 208 \\
\hline 894 & 5,889 & 27 & 10,711 & 164 & & 10,877 & 12,0100 & 8534 & 279 & 213 \\
\hline 927 & 8,796 & 31 & $2 i, 359$ & 191 & & 21,550 & 13,832 & 10,308 & 291 & 223 \\
\hline 8,015 & 7,811 & 32 & 21, (1) & 197 & & 22,005 & 13,762 & 10,187 & 316 & 232 \\
\hline 906 & 8,717 & 30 & 20,100 & 184 & & 20,564 & 12,454 & 0,981 & 274 & 198 \\
\hline 1,010 & 8,736 & 33 & 22,341 & 203 & & 22,544 & 13,242 & 8,367 & 29 & 212 \\
\hline 1,097 & 10,023 & 35 & 23,555 & 215 & & 23,770 & 13,556 & 8,407 & 310 & 215 \\
\hline 1,083 & 11,906 & 39 & 23,450 & 215 & & 23,665 & 13,303 & 8,918 & 300 & 204 \\
\hline 900 & 12,814 & 29 & 19,372 & 178 & & 19,550 & 10,508 & 7,017 & 244 & 163 \\
\hline 850 & 13,684 & 28 & 18,648 & 172 & & 18,820 & 9,822 & 8,434 & 222 & 148 \\
\hline 1,135 & 18,799 & 37 & 24,531 & 227 & & 24,758 & 12,545 & 8,081 & 2 & 185 \\
\hline \multirow{15}{*}{750} & 15,5199 & 24 & 15,864 & 148 & & 16,012 & 7,877 & 9,985 & 104 & 116 \\
\hline & & (13) & & & 220 & 220 & 105 & 85 & & \\
\hline & & (50) & & & mol & 996 & 483 & 281 & & \\
\hline & & (62) & & & 1,098 & 1,040 & 472 & 281 & & \\
\hline & & $(81)$ & & & 1,349 & 1,369 & 599 & 349 & & \\
\hline & & (65) & & & 1,099 & 1,098 & 486 & 267 & & \\
\hline & & $(67)$ & & & 1.193 & 1,133 & $\$ 87$ & 282 & & \\
\hline & & (78) & & & 1,319 & 1,318 & 527 & 291 & & \\
\hline & & (72) & & & 1,217 & 1,217 & 473 & 255 & & \\
\hline & & (9) & & & 152 & 152 & 57 & 30 & & \\
\hline & & 504 & 350,250 & 3,098 & 0,555 & 381,904 & 231,065 & 175,008 & 4,943 & 3,747 \\
\hline & & & 225,461 & 1,975 & 3,629 & & 231,065 & & & \\
\hline & & & $\$ 45.6$ & 8.4 & 8.7 & & 946.7 & & & \\
\hline & & & 172,227 & 1,498 & 2,018 & & & 175,800 & & \\
\hline & & & $\$ 196.0$ & 8.4 & 1.6 & & & 846.8 & & \\
\hline
\end{tabular}


TAบน $6-15$

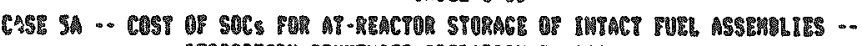

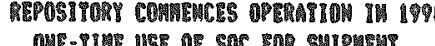

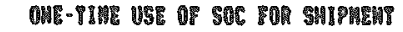

(502 of aR Sropage Requiraments Provided by Castes)

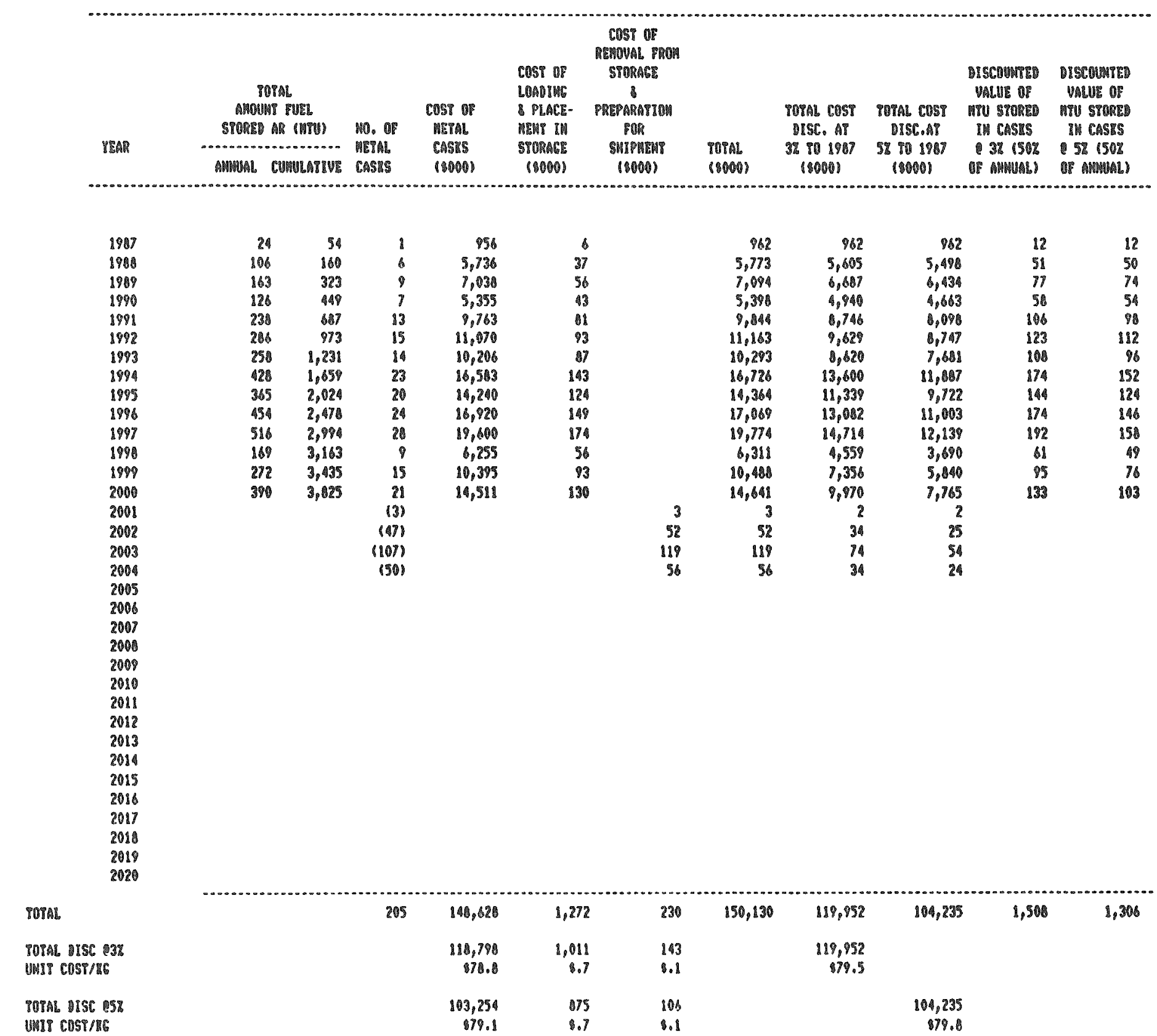


TARLE C-16

CASE 58 -. COST OP SOCS FOR MT-REACTOR STORAGE OO IMTACT PUEL ASSEMULIES -. REPSITORY COWWEMCES OPRATIOM IVI 2003

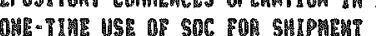

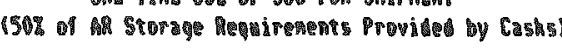

\begin{tabular}{|c|c|c|c|c|c|c|c|c|c|c|}
\hline \multirow[b]{4}{*}{ YISAR } & \multirow{5}{*}{\multicolumn{2}{|c|}{ 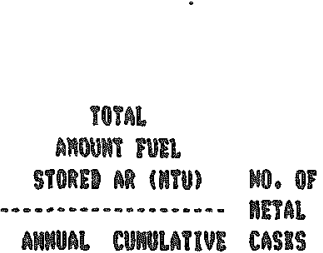 }} & \multirow{4}{*}{$\begin{array}{l}\text { COST OF } \\
\text { MBTAL } \\
\text { CASES } \\
\text { (BOAOO) }\end{array}$} & \multicolumn{3}{|c|}{ 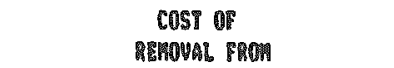 } & \multirow{4}{*}{$\begin{array}{l}\text { TOTAL. COST } \\
\text { ISC. AT } \\
\text { ME T0 1987 }\end{array}$} & \multirow{5}{*}{$\begin{array}{c}\text { POTML COST } \\
\text { DISC.AI } \\
58 \text { T0 1967 } \\
\text { (1900) }\end{array}$} & \multirow{5}{*}{ 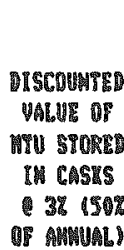 } & \multirow{5}{*}{ 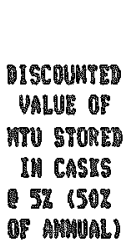 } \\
\hline & & & & COST of & ST0RAc & & & & & \\
\hline & & & & 8 PLACE- & $\begin{array}{c}\text { PMEPARATIO } \\
\text { ROR }\end{array}$ & & & & & \\
\hline & & & & ST0RAอE & SHIPMEMT & TOrAL, & & & & \\
\hline & & & & (1000) & $(8000)$ & $80000)$ & (8000) & & & \\
\hline
\end{tabular}

\begin{tabular}{|c|c|c|c|}
\hline 24 & 54 & 1 & 956 \\
\hline 106 & 160 & 6 & 9,738 \\
\hline 163 & 323 & 9 & 7,038 \\
\hline 128 & 849 & 7 & 5,355 \\
\hline 238 & 607 & 13 & 8,763 \\
\hline 286 & 773 & 15 & 11,070 \\
\hline 258 & 1,231 & 14 & 10,206 \\
\hline 428 & 1,058 & 23 & $166_{8} 583$ \\
\hline 365 & 2,026 & 20 & 11,240 \\
\hline 454 & 2,478 & 24 & 16,020 \\
\hline 518 & 2,994 & 20 & 19,600 \\
\hline 589 & 3,563 & 31 & 21,483 \\
\hline 472 & 4,235 & 36 & 24,788 \\
\hline 790 & 5,025 & 43 & 27,388 \\
\hline 144 & 5,080 & 45 & 30,510 \\
\hline 927 & 8,708 & 50 & 33,650 \\
\hline 615 & 7,411 & 33 & 22,077 \\
\hline 506 & 7,017 & 26 & 17,342 \\
\hline 819 & 1,538 & 33 & 21,912 \\
\hline \multirow[t]{8}{*}{187} & 8,723 & 10 & 8,630 \\
\hline & & (38) & \\
\hline & & $(92)$ & \\
\hline & & (94) & \\
\hline & & $(79)$ & \\
\hline & & $(7 / 3)$ & \\
\hline & & (87) & \\
\hline & & 18 & \\
\hline
\end{tabular}

37
37
56
93
11
93
67
149
124
149
174
192
223
267
279
310
205
181
205
62

\begin{tabular}{|c|c|}
\hline & 962 \\
\hline & 5,773 \\
\hline & 7,094 \\
\hline & 5,398 \\
\hline & 9,844 \\
\hline & 11,163 \\
\hline & 10,293 \\
\hline & 16,728 \\
\hline & 16,364 \\
\hline & 17,069 \\
\hline & 18,774 \\
\hline & 21,875 \\
\hline & $20_{8} 011$ \\
\hline & 29,636 \\
\hline & 30,788 \\
\hline & 33,960 \\
\hline & 22,282 \\
\hline & 17,503 \\
\hline & 23,117 \\
\hline & 8,692 \\
\hline 42 & 42 \\
\hline 102 & 102 \\
\hline 104 & 104 \\
\hline 酙 & $A$ \\
\hline 81 & 81 \\
\hline 97 & 97 \\
\hline 9 & 8 \\
\hline
\end{tabular}

\begin{tabular}{|c|c|c|c|}
\hline $96 \%$ & 962 & 12 & 12 \\
\hline 5,605 & 5,698 & 51 & 50 \\
\hline 6,687 & 6,434 & 77 & 74 \\
\hline 9,840 & 9,863 & 58 & $5 A$ \\
\hline 8,796 & 0,098 & 108 & 80 \\
\hline 9,629 & 4, 797 & 123 & 112 \\
\hline 8,820 & 7,681 & 108 & 86 \\
\hline 13,600 & 11,97 & 1779 & 852 \\
\hline 11,339 & 0,722 & 144 & 124 \\
\hline 13,082 & 18,003 & 174 & 14. \\
\hline 19,714 & 12,138 & 172 & 154 \\
\hline 15,658 & 12,673 & 208 & 100 \\
\hline 17,528 & 13,918 & 236 & 197 \\
\hline 20,281 & 15,716 & 289 & 209 \\
\hline 20,355 & 15,551 & 279 & 213 \\
\hline 21,798 & 16,335 & 298 & 223 \\
\hline 13,025 & 10,200 & 182 & 101 \\
\hline 10,590 & 7,637 & 153 & 110 \\
\hline 12,991 & 9,190 & 192 & 129 \\
\hline 3,016 & 2,846 & 53 & 97 \\
\hline 23 & 16 & & \\
\hline 55 & 37 & & \\
\hline 55 & 36 & & \\
\hline 44 & 28 & & \\
\hline 40 & 25 & & \\
\hline 46 & 20 & & \\
\hline 4 & 3 & & \\
\hline
\end{tabular}

$\begin{array}{rrr}325,200 & 2,397 & 523 \\ 232,669 & 2,059 & 268 \\ 175.4 & 8.7 & 3.1 \\ 199,005 & 1,684 & 173 \\ 175.8 & 1.7 & 1.1\end{array}$

328,628

234,995

190,362

3,080

2,498

POTAL ISC

TOTAL DISC O5\%

190,282 
TAMLE [-1)

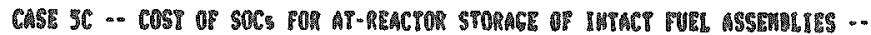

REPOSI TORY CONEEXESS OP ERATIOH IH 2000

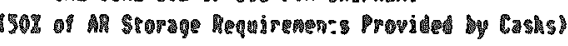

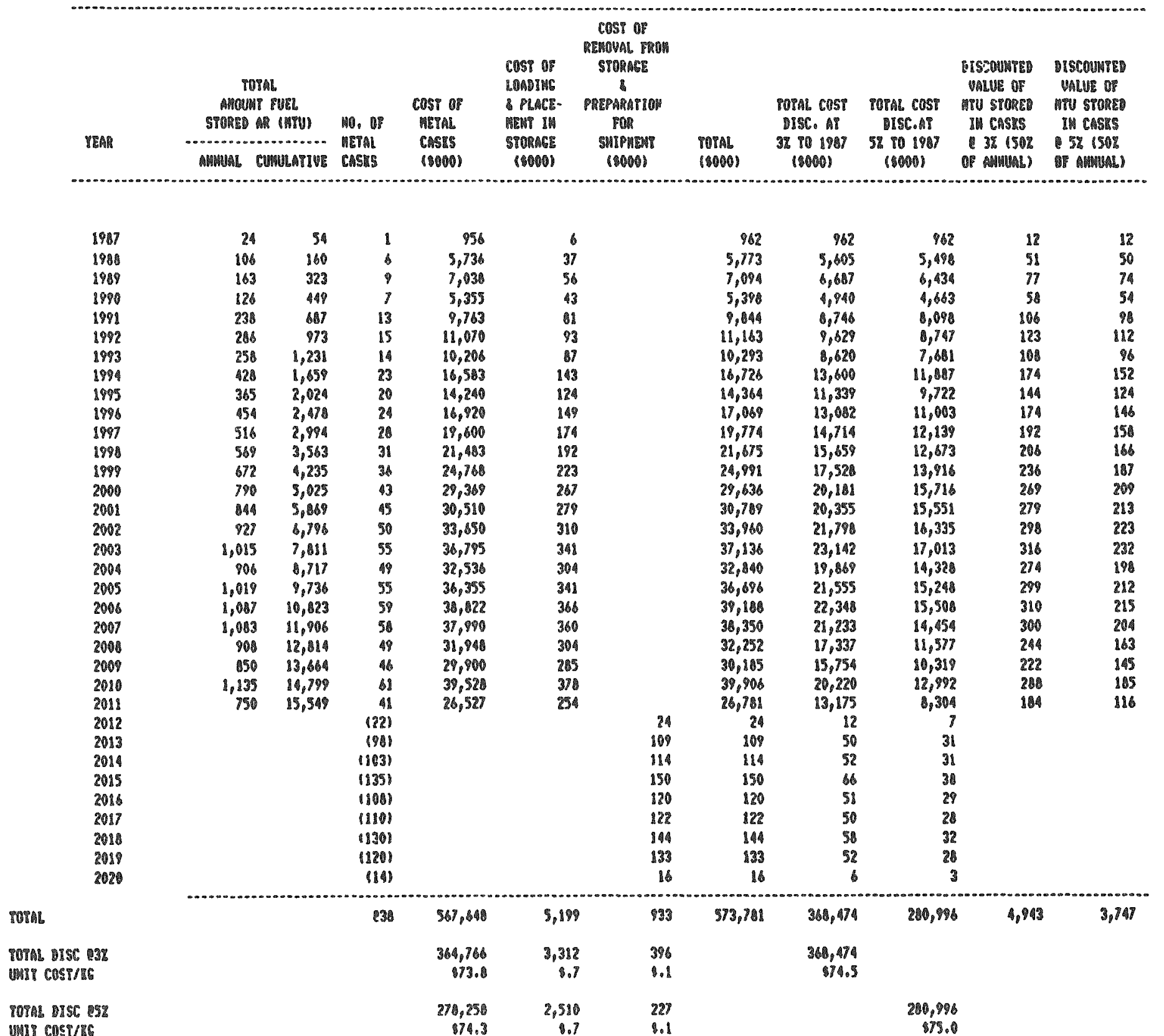




\section{TAL}

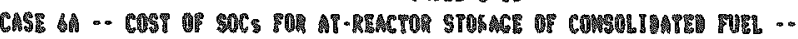

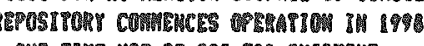

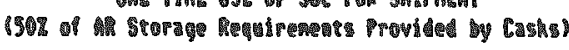

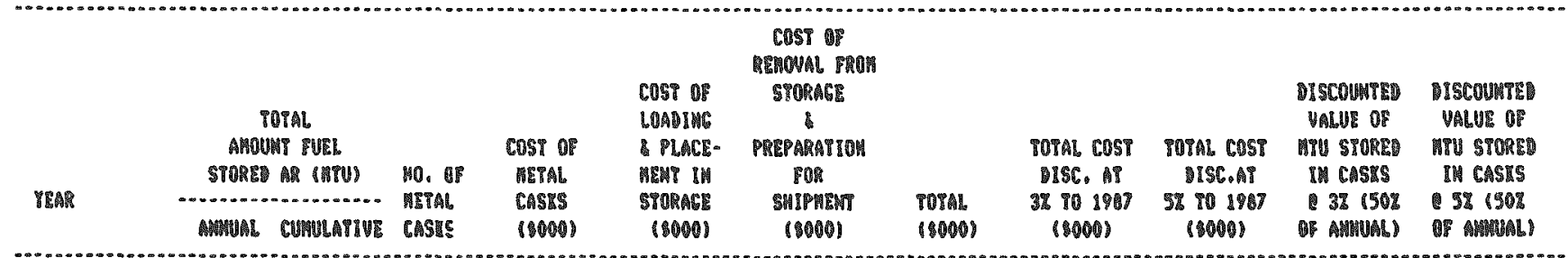

\begin{tabular}{|c|c|c|c|c|c|c|c|c|c|c|c|}
\hline 1987 & 24 & 54 & 1 & 956 & 6 & & 982 & 982 & 962 & 12 & 12 \\
\hline 1940 & 106 & 180 & 4 & 3,824 & 25 & & 3,849 & 3,736 & 3,665 & 51 & 50 \\
\hline 1989 & 183 & 323 & 5 & 4,700 & 31 & & 4811 & 4535 & 4363 & $n$ & 74 \\
\hline 1990 & 126 & 449 & 4 & 3,120 & 25 & & 3,153 & 2,085 & 2,723 & 58 & $5 A$ \\
\hline 1991 & 238 & 687 & 7 & 5,390 & 4 & & 5,433 & 4,827 & $4: 970$ & 106 & 98 \\
\hline 1992 & 284 & 973 & 10 & 7,560 & 81 & & $7, \sqrt{2} 2$ & 8,574 & 5,972 & 123 & 112 \\
\hline 1903 & 258 & 1,231 & 8 & 5,860 & 48 & & 8,017 & 9,038 & 4,490 & 100 & 86 \\
\hline 1994 & 928 & 1,659 & 14 & 10,310 & 86 & & 10,404 & 0,458 & 7,394 & 174 & 152 \\
\hline 1095 & 365 & 2,024 & 12 & 8,740 & 74 & & 0,822 & 6,964 & 5871 & 140 & 124 \\
\hline 1996 & 454 & 2,470 & 15 & 10,845 & 92 & & 10,937 & 8,302 & 7,050 & 174 & 146 \\
\hline 1997 & 516 & 2,994 & 17 & 12,172 & 105 & & 12,277 & 9,135 & 7,537 & 192 & 150 \\
\hline 1998 & 169 & 3,163 & g & 3,550 & 31 & & 3,591 & 2,594 & 2,099 & 11 & 4 \\
\hline 1999 & 272 & 3,435 & 9 & 6,390 & $\$ 5$ & & 6,845 & 4,521 & 3,589 & 95 & 78 \\
\hline 2000 & 390 & 3,625 & 18 & 0,472 & 74 & & 8,594 & $\$, 019$ & 1,592 & 193 & 103 \\
\hline 2001 & & & (1) & & & 1 & 1 & 1 & 1 & & \\
\hline 2002 & & & (291 & & & 36 & 36 & 23 & 17 & & \\
\hline 2003 & & & (84) & & & 79 & 79 & 49 & 36 & & \\
\hline 2004 & & & 1301 & & & 37 & 37 & 22 & 16 & & \\
\hline \multirow{2}{*}{\multicolumn{12}{|c|}{$\begin{array}{l}2005 \\
2005\end{array}$}} \\
\hline \multirow{2}{*}{\multicolumn{12}{|c|}{2007}} \\
\hline & & & & & & & & & & & \\
\hline \multicolumn{12}{|l|}{2000} \\
\hline \multicolumn{12}{|l|}{2009} \\
\hline \multirow{2}{*}{\multicolumn{12}{|c|}{$\begin{array}{l}2010 \\
2011\end{array}$}} \\
\hline & & & & & & & & & & & \\
\hline \multicolumn{12}{|l|}{2012} \\
\hline \multicolumn{12}{|l|}{2013} \\
\hline \multicolumn{12}{|l|}{2014} \\
\hline \multirow{2}{*}{\multicolumn{12}{|c|}{$\begin{array}{l}2015 \\
2016\end{array}$}} \\
\hline & & & & & & & & & & & \\
\hline \multicolumn{12}{|l|}{2017} \\
\hline \multicolumn{12}{|l|}{2018} \\
\hline \multirow{2}{*}{\multicolumn{12}{|c|}{$\begin{array}{l}2010 \\
2020\end{array}$}} \\
\hline & & & & & & & & & & & \\
\hline & & & 123 & 2,111 & 796 & 152 & 93,020 & 74,529 & 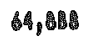 & 1,500 & 1,308 \\
\hline (3) & & & & 73,1932 & 802 & 85 & & 81,529 & & & \\
\hline 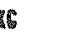 & & & & 1490.0 & $\$ .4$ & 8.1 & & 949.4 & & & \\
\hline BS\% & & & & $\$ 4,296$ & $\$ 22$ & 70 & & & 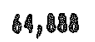 & & \\
\hline 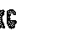 & & & & 899.2 & 8.4 & 1.1 & & & 848.7 & & \\
\hline
\end{tabular}


TALL C - 19

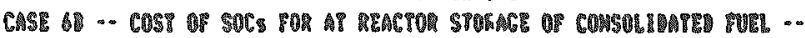

$$
\text { REPOSIYORY COHFEMCES OPERTIIOY IN } 2003
$$

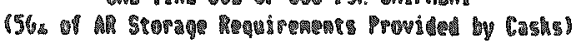

\begin{tabular}{|c|c|c|c|c|c|c|c|c|c|c|}
\hline \multirow[b]{4}{*}{ 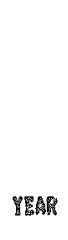 } & \multicolumn{10}{|c|}{$\begin{array}{l}\text { Cost OP } \\
\text { REmoval 8Reห }\end{array}$} \\
\hline & POPAL & & & $\begin{array}{l}\text { COSP OP } \\
\text { LOAB INE }\end{array}$ & 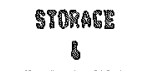 & & & & $\begin{array}{c}\text { DISCOUMTE } \\
\text { VALUE OP }\end{array}$ & 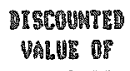 \\
\hline & 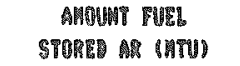 & MOB. & $\begin{array}{l}\text { COSP MP } \\
\text { METAL }\end{array}$ & $\begin{array}{l}\text { DLACF- } \\
\text { MEMT IM }\end{array}$ & $\begin{array}{l}\text { PUEPARATIOM } \\
\text { FOA }\end{array}$ & & $\begin{array}{l}\text { TOTAL CUST } \\
\text { DISC. AT }\end{array}$ & $\begin{array}{l}\text { POTAL COST } \\
\text { MISC.AT }\end{array}$ & $\begin{array}{l}\text { MTU SPQRER } \\
\text { M CASE }\end{array}$ & $\begin{array}{l}\text { MVU STOME } \\
\text { IW CASIS }\end{array}$ \\
\hline & 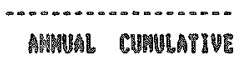 & $\begin{array}{l}\text { MEYAL } \\
\text { CASAE }\end{array}$ & $\begin{array}{l}\text { CASRS } \\
\text { (1000) }\end{array}$ & 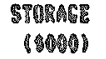 & 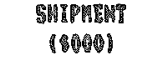 & $\begin{array}{l}\text { T0TaL } \\
\text { 18000) }\end{array}$ & $\begin{array}{c}38.9087 \\
(2000)\end{array}$ & $\begin{array}{c}528101907 \\
(0060)\end{array}$ & 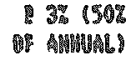 & 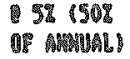 \\
\hline
\end{tabular}

1997
1988
1999
1990
1991
1992
1993
1994
1995
1996
1997
1998
1999
2000
2001
2002
2003
2004
2005
2006
2007
2008
2009
2010
2011
2012
2013
2014
2015
2016
2017
2010
2019
2002

\begin{tabular}{|c|c|}
\hline 24 & 54 \\
\hline 106 & 160 \\
\hline 183 & 323 \\
\hline 126 & 448 \\
\hline 238 & 687 \\
\hline 280 & 913 \\
\hline 850 & 1,231 \\
\hline 120 & 1,659 \\
\hline 365 & 2,024 \\
\hline 454 & 2,470 \\
\hline 516 & 2,998 \\
\hline 569 & 3,583 \\
\hline 672 & 4,235 \\
\hline 790 & 5,025 \\
\hline 54 & 5,889 \\
\hline 927 & 6,796 \\
\hline 615 & 7,411 \\
\hline 506 & 7,917 \\
\hline 819 & 8,538 \\
\hline 197 & 1,723 \\
\hline
\end{tabular}

\begin{tabular}{|c|c|c|}
\hline 1 & 856 & 6 \\
\hline 4 & 3,024 & 25 \\
\hline 5 & 4,700 & 31 \\
\hline 4 & 3,128 & 25 \\
\hline 7 & 5,390 & 43 \\
\hline 10 & 7,500 & $\$ 1$ \\
\hline \& & 5,840 & 99 \\
\hline 14 & 10,310 & 26 \\
\hline 12 & 8,740 & 74 \\
\hline 15 & 10,045 & 82 \\
\hline 17 & 12,172 & 105 \\
\hline 18 & 12,700 & 111 \\
\hline 22 & $15, \mathrm{Aes}$ & 135 \\
\hline 26 & 10,170 & 180 \\
\hline 27 & 10,711 & 166 \\
\hline 31 & 21,359 & 191 \\
\hline 20 & 13,700 & 123 \\
\hline 17 & 11,811 & 105 \\
\hline 20 & 13,820 & 123 \\
\hline 8 & 9,074 & 37 \\
\hline
\end{tabular}

28
68
68
58
54
64
6

\begin{tabular}{|c|c|}
\hline 962 & \\
\hline 3,849 & \\
\hline 4,811 & \\
\hline 3,153 & \\
\hline 5,133 & \\
\hline 7,621 & \\
\hline 6,0017 & \\
\hline 10,404 & \\
\hline 9,822 & \\
\hline 10,937 & \\
\hline 12,277 & \\
\hline 12,891 & \\
\hline 15,623 & \\
\hline 18,334 & \\
\hline 18,977 & \\
\hline 24,550 & \\
\hline 13,823 & \\
\hline 11,718 & \\
\hline 13,743 & \\
\hline 4,111 & \\
\hline 28 & \\
\hline 68 & \\
\hline 70 & \\
\hline 58 & \\
\hline 54 & \\
\hline 64 & \\
\hline 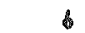 & \\
\hline
\end{tabular}

\begin{tabular}{|c|c|}
\hline 982 & 828 \\
\hline 3,736 & 3,865 \\
\hline 4,535 & 4,383 \\
\hline 2,805 & 2,723 \\
\hline 4,527 & 4,870 \\
\hline 6,574 & 5,972 \\
\hline 5,039 & 9,490 \\
\hline 8,458 & 7,394 \\
\hline 6,984 & 5,978 \\
\hline 0,302 & 7,050 \\
\hline 9,135 & 7,537 \\
\hline 8,312 & 7,537 \\
\hline 10,858 & 8,700 \\
\hline 12,484 & 9,723 \\
\hline 12,480 & 8,534 \\
\hline 13,632 & 10,366 \\
\hline 8,814 & 0,332 \\
\hline 7,008 & 9,111 \\
\hline 9,073 & 5,710 \\
\hline 2,344 & 1,627 \\
\hline 16 & 11 \\
\hline 36 & 24 \\
\hline 37 & 24 \\
\hline 30 & 19 \\
\hline$y$ & 18 \\
\hline 31 & 19 \\
\hline 3 & 2 \\
\hline
\end{tabular}

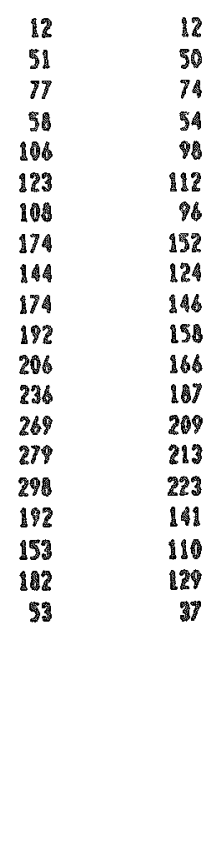

TUTML

ropal DISC 935 แม⿱乛龰 COST

PTPL DIS 452 and costrite

\begin{tabular}{|c|c|c|c|c|c|c|c|c|}
\hline 284 & 203,200 & 1,748 & 349 & 205,301 & 1106 & 119,354 & 3,086 & 2,693 \\
\hline & 145,44 & 1,239 & 178 & & $14 B_{p}$ & & & \\
\hline & 047.1 & 6.4 & 8.1 & & 347.6 & & & \\
\hline & 180,236 & 1,002 & 115 & & & 118,394 & & \\
\hline & 47.1 & 8.4 & 8.0 & & & 897.8 & & \\
\hline
\end{tabular}




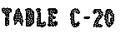

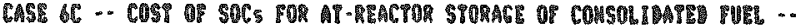

REPOSIYGRY COMALMCES OPERATIOH IH 2008

II/ $3 / 1907$

OHE-

150\% of al Storage Rewar renents Provided by Casks?

\begin{tabular}{|c|c|c|c|c|c|c|c|c|c|c|}
\hline \multirow[t]{2}{*}{ PEAR } & \multirow{2}{*}{\multicolumn{2}{|c|}{$\begin{array}{c}\text { TDTAL } \\
\text { AMOUT FUEL } \\
\text { STORED AR (MTU) }\end{array}$}} & \multirow[t]{2}{*}{$\begin{array}{l}\text { COST OF } \\
\text { METAL } \\
\text { CASES } \\
\text { (BOOO) }\end{array}$} & $\begin{array}{l}\text { COST OP } \\
\text { LOADYNC } \\
\text { PLACE- } \\
\text { PEET IN } \\
\text { STORACE }\end{array}$ & 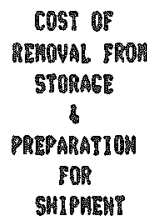 & & \multirow[t]{2}{*}{$\begin{array}{l}\text { TOTAL COST } \\
\text { DISG. AT } \\
\text { 32 T0 } 1987 \\
\text { (6000) }\end{array}$} & \multirow{2}{*}{$\begin{array}{c}\text { T0TAL COST } \\
\text { ISC.AT } \\
\text { 52 T0 1997 } \\
\text { (5000) }\end{array}$} & \multirow[t]{2}{*}{ 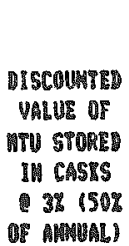 } & \multirow[t]{2}{*}{ 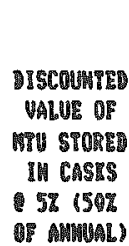 } \\
\hline & & & & $(8000)$ & $(1000)$ & 880001 & & & & \\
\hline
\end{tabular}

\begin{tabular}{|c|c|}
\hline 24 & 54 \\
\hline 106 & 160 \\
\hline 163 & 323 \\
\hline 126 & 449 \\
\hline 238 & 697 \\
\hline 206 & 973 \\
\hline 251 & 1,231 \\
\hline 928 & 1,859 \\
\hline 365 & 2,024 \\
\hline 454 & 2,470 \\
\hline$\$ 11$ & 2,994 \\
\hline 569 & 3,563 \\
\hline 672 & 4,235 \\
\hline 790 & 5,028 \\
\hline 844 & 5,860 \\
\hline 927 & 0,796 \\
\hline 1,015 & 7,811 \\
\hline 906 & 8,717 \\
\hline 1,019 & 9,736 \\
\hline 1,087 & 10,123 \\
\hline 1,083 & 11,900 \\
\hline 908 & 12,814 \\
\hline 550 & 13,864 \\
\hline 1,135 & 14,799 \\
\hline 750 & 15,509 \\
\hline
\end{tabular}

TOT)

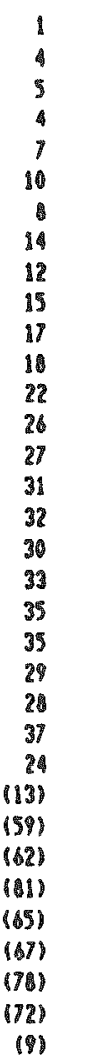

\section{3,956}

4780

3,128

5,390

3,968

10,318

8,748

10,145

12,172
12,780

12,480

18,178

18,711

21,359

21,380

20,400

22,341
23,555

23,555
23,450

10,372

18,648

24,531

6
25
31
25
43
61
19
8
71
92
105
111
195
160
166
191
197
184
203
215
215
178
172
227
196

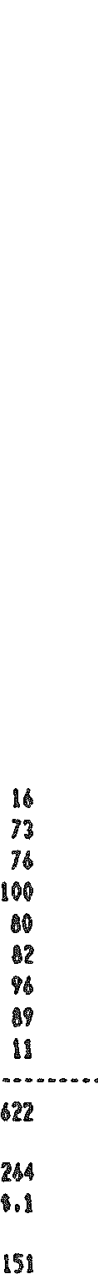

\begin{tabular}{|c|c|c|c|c|}
\hline 962 & 962 & 842 & 12 & 12 \\
\hline 3,849 & 3,736 & 3,665 & 51 & 50 \\
\hline 1,811 & 4,535 & 4,363 & $n$ & 74 \\
\hline 3,153 & 2,885 & 2,723 & 58 & 54 \\
\hline 5,433 & 4,827 & 4,470 & 106 & 98 \\
\hline 7,621 & 6,574 & 5,972 & 123 & 112 \\
\hline 6,017 & 5,039 & 4,490 & 100 & 96 \\
\hline 10,404 & 9,959 & 7,394 & 174 & 152 \\
\hline 1,822 & 6,964 & 5,971 & 144 & 124 \\
\hline 10,937 & 1,382 & 7,050 & 174 & 148 \\
\hline 12,277 & 9,135 & 7,537 & 192 & 158 \\
\hline 12,891 & 9,312 & 7,537 & 206 & 168 \\
\hline 15,623 & 10,858 & 8,700 & 236 & 887 \\
\hline 18,334 & 12,844 & 9,723 & 268 & 209 \\
\hline 18,877 & 12,480 & 9,534 & 279 & 213 \\
\hline 21,550 & 13,832 & 10,366 & 298 & 223 \\
\hline 22,085 & 13,762 & 10,117 & 316 & 232 \\
\hline 20,584 & 12,454 & 891 & 274 & 198 \\
\hline 22,544 & 13,242 & 9,367 & 299 & 212 \\
\hline 23,770 & 12,556 & 9,407 & 310 & 215 \\
\hline 23,655 & 13,103 & 0,818 & 300 & 204 \\
\hline 19,550 & 10,509 & 7,017 & 244 & 163 \\
\hline 18,020 & 9,322 & 6,434 & 222 & 165 \\
\hline 24,758 & 12,545 & 8,061 & 288 & 185 \\
\hline 18,012 & 7,977 & 4,965 & 184 & 116 \\
\hline 16 & 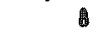 & 5 & & \\
\hline 73 & 34 & 20 & & \\
\hline 76 & 34 & 20 & & \\
\hline 100 & 44 & 25 & & \\
\hline 80 & 34 & 19 & & \\
\hline 82 & 34 & 18 & & \\
\hline 98 & 38 & 21 & & \\
\hline 89 & 34 & 18 & & \\
\hline 11 & 4 & 2 & & \\
\hline \multirow[t]{3}{*}{$33_{8}, 971$} & 227,700 & 173,178 & 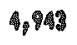 & 3,747 \\
\hline & $\begin{array}{r}227_{7} 700 \\
546,1\end{array}$ & & & \\
\hline & & 173,976 & & \\
\hline
\end{tabular}

TOTAL DISE B

UMIS COST/VG

$\$ 48.0$

8.4

$\$ .0$

14. 
TABLE C-ZI

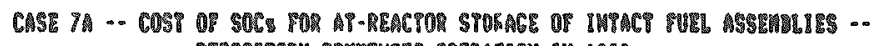

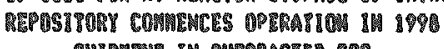

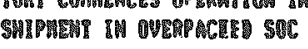

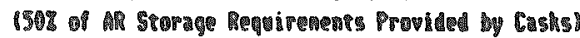

\begin{tabular}{|c|c|c|c|c|c|c|c|c|c|c|}
\hline \multirow[t]{2}{*}{ 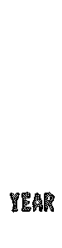 } & \multirow{2}{*}{\multicolumn{2}{|c|}{ 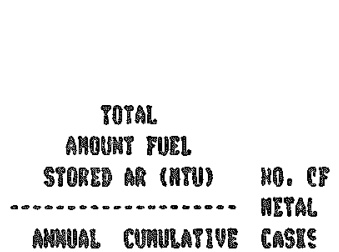 }} & \multirow[t]{2}{*}{$\begin{array}{l}\text { COST OP } \\
\text { WEFAL } \\
\text { CASES } \\
\text { TMOOU }\end{array}$} & $\begin{array}{l}\text { COSY OF } \\
\text { LOADIRE } \\
\text { 8 PLACE- } \\
\text { MEMT II } \\
\text { SPURACE }\end{array}$ & 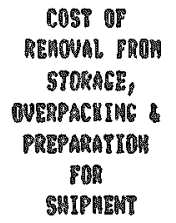 & & $\begin{array}{l}\text { POTAL COST } \\
\text { DISC. AT } \\
\text { 3\% \%O } 1967\end{array}$ & $\begin{array}{l}\text { TOTAL CAST } \\
\text { UISC.AT } \\
\text { 5\% TO } 1987\end{array}$ & 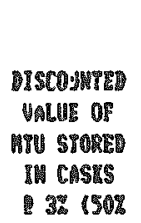 & 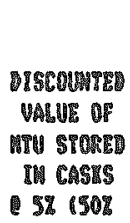 \\
\hline & & & & 80001 & $(0000)$ & 880008 & 80000 & (nocos) & 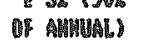 & OE AMENAL \\
\hline
\end{tabular}

\begin{tabular}{|c|c|c|c|c|c|c|c|c|c|c|}
\hline 24 & 58 & 1 & 958 & \& & & 912 & 862 & 962 & 12 & 12 \\
\hline 100 & 160 & 8 & 5,738 & 37 & & 5,773 & 5,605 & 5,491 & 51 & 50 \\
\hline 163 & 323 & 8 & 7,030 & 56 & & 7,004 & 6,697 & 6,434 & 77 & 74 \\
\hline 126 & 489 & 7 & 5,355 & 43 & & 5,396 & 4,940 & 4,863 & 58 & 54 \\
\hline 238 & 687 & 13 & 9,763 & 81 & & 9,844 & 8,746 & 0,090 & 106 & 98 \\
\hline 286 & 973 & 15 & 11,070 & 93 & & 11,163 & 9.629 & 0,797 & 123 & 112 \\
\hline 25\% & 8,231 & 14 & 10,206 & 97 & & 10,293 & 0,820 & 7,681 & 108 & 86 \\
\hline 428 & 1,659 & 23 & 16,583 & 143 & & 16,726 & 13,600 & 11,887 & 178 & 152 \\
\hline 365 & 2,024 & 20 & 14,210 & 124 & & 14,364 & 11,339 & 0,722 & 164 & 124 \\
\hline 454 & $2_{8} 478$ & 28 & 16,720 & 149 & & 17,060 & 13,062 & 11,003 & 174 & 146 \\
\hline 516 & 2,990 & 28 & 19,800 & 874 & & 19,774 & 84,711 & 12,139 & 102 & 158 \\
\hline 169 & 3,163 & 9 & $0_{1}^{2} 255$ & 56 & & 6,311 & 7.55\% & 3,690 & 68 & 48 \\
\hline 272 & 3,435 & 15 & 10,395 & 9 & & 10,688 & 7,356 & 5,840 & 95 & is \\
\hline \multirow[t]{5}{*}{39} & 3,823 & 21 & 14,511 & 130 & & 10,641 & 9,970 & 7,769 & 138 & 103 \\
\hline & & (3) & & & 21 & 21 & it & 11 & & \\
\hline & & $(177)$ & & & 335 & 395 & 215 & 101 & & \\
\hline & & 1107 & & & 783 & 763 & 475 & 350 & & \\
\hline & & 1501 & & & 357 & 357 & 2116 & 158 & & \\
\hline
\end{tabular}

$295 \quad 146,620$

1,272

1,476

151,376

120,729

109600

1,596

1,306

จตกล แIS อ3ร

UसI $\cos / /$ Tล

T07月 OISC $25 \%$

UมTY CDST/ /

$\begin{array}{rrr}118,796 & 1,011 & 820 \\ 070.0 & 8.7 & 8.6 \\ 103,258 & 075 & 677\end{array}$

120,729

03,254

6.8

678

104,800 
THALE C-22

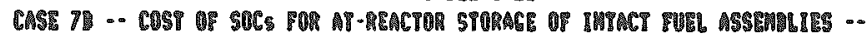

REPQSI YORY CONAEWCES OPERATIOH IH 2003

11/ $4 / 1987$ SHIPWEMT IN WVERPACRE SUCS

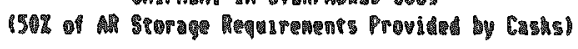

\begin{tabular}{|c|c|c|c|c|c|c|c|c|c|c|}
\hline \multirow{2}{*}{ rem } & \multirow{2}{*}{\multicolumn{2}{|c|}{ 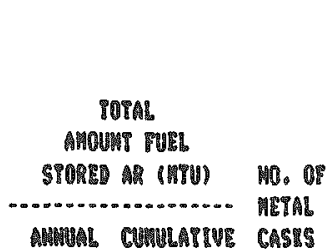 }} & \multirow{2}{*}{$\begin{array}{l}\text { COST 0? } \\
\text { HETAL } \\
\text { CASTS } \\
\text { (1000) }\end{array}$} & 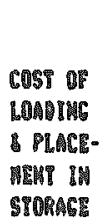 & 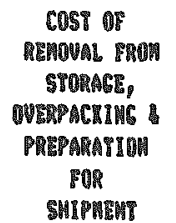 & TอిAน & \multirow{2}{*}{ 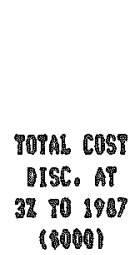 } & \multirow{2}{*}{ 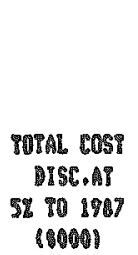 } & \multirow[t]{2}{*}{ 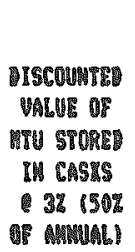 } & \multirow[t]{2}{*}{ 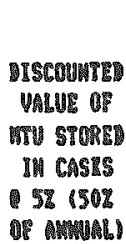 } \\
\hline & & & & 180001 & 100001 & (80001) & & & & \\
\hline
\end{tabular}

\begin{tabular}{|c|c|c|c|c|c|c|c|c|c|c|c|}
\hline 2987 & 24 & 54 & 1 & 958 & 6 & & 962 & 962 & 982 & 12 & 12 \\
\hline 1988 & 106 & 160 & 6 & 3,736 & 37 & & 5,773 & 5,605 & 5,490 & 51 & 30 \\
\hline 1989 & 163 & 323 & 9 & 7,038 & 58 & & 7.094 & 6,687 & 6,434 & $\eta$ & 74 \\
\hline 1990 & 126 & 449 & 7 & 5,355 & 43 & & 5,398 & 4,940 & 4,683 & 58 & 54 \\
\hline 1991 & 238 & เ89 & 19 & 9,783 & 1 & & 8,824 & 0,746 & 8,096 & 108 & 98 \\
\hline 1998 & 280 & 773 & 15 & 81,070 & 93 & & 11,163 & 9,629 & 8,797 & 123 & 112 \\
\hline 2983 & 258 & 1,231 & 14 & 10,208 & 97 & & 10,293 & 0,820 & 7,881 & 108 & 98 \\
\hline 1994 & 421 & 1,659 & 23 & 16,583 & 143 & & 16,728 & 13,600 & 11,127 & $\$ 74$ & 152 \\
\hline 1095 & 365 & 2,024 & 20 & 118,200 & 124 & & 14,364 & 11,339 & 9,722 & 144 & 124 \\
\hline 1996 & 454 & 2,478 & 24 & 18,920 & 149 & & 17,089 & 13,002 & 11,003 & 174 & 148 \\
\hline 1997 & 516 & 2,994 & 20 & 19,600 & 274 & & $19,77 \mathrm{~A}$ & 10,714 & 12,139 & 192 & 15\% \\
\hline 1998 & $58 \%$ & 3,563 & 31 & 21,403 & 192 & & 21,675 & $15 \%, 659$ & 12,673 & 200 & 168 \\
\hline 1989 & 872 & 4,235 & 36 & 20,760 & 223 & & 24,911 & 17,520 & 13,914 & 236 & 187 \\
\hline 2000 & 790 & 5,025 & 43 & 29,369 & 267 & & 29,836 & 20,101 & 15,718 & 268 & 208 \\
\hline 2001 & 8.44 & 5,888 & 45 & 30,510 & 279 & & 30,789 & 20,355 & 15,551 & $27 \%$ & 213 \\
\hline 2002 & 927 & 8796 & 50 & 33,850 & 310 & & 33,980 & 21,798 & 16,335 & 278 & 223 \\
\hline 2003 & 615 & 7,411 & 33 & 22,077 & 205 & & 22,282 & 13,885 & 10,208 & 192 & 141 \\
\hline 2004 & 508 & 7,917 & 26 & 17,342 & 161 & & 27,503 & 10,590 & 7,637 & 153 & 110 \\
\hline 2005 & (19) & 8536 & 33 & 21,12 & 205 & & 22,117 & 12,991 & 9,190 & 192 & 129 \\
\hline 2006 & 187 & 8,723 & 10 & 0,830 & 6 & & 8,692 & 3,118 & 2,840 & 53 & 37 \\
\hline 2007 & & & $(38)$ & & & 271 & 271 & 150 & 102 & & \\
\hline 2008 & & & $(82)$ & & & 656 & 856 & 353 & 235 & & \\
\hline 2009 & & & (94) & & & 870 & 670 & 350 & 229 & & \\
\hline 2010 & & & $(79)$ & & & 563 & 583 & 205 & 183 & & \\
\hline 2011 & & & $(7 / 3)$ & & & 528 & 521 & 258 & 181 & & \\
\hline 2012 & & & $(87)$ & & & 620 & 620 & 298 & 103 & & \\
\hline 2013 & & & (10) & & & 57 & 57 & 26 & 16 & & \\
\hline \multicolumn{12}{|l|}{2014} \\
\hline \multirow{2}{*}{\multicolumn{12}{|c|}{2015}} \\
\hline & & & & & & & & & & & \\
\hline \multicolumn{12}{|l|}{2017} \\
\hline \multicolumn{12}{|l|}{2010} \\
\hline \multirow{2}{*}{\multicolumn{12}{|c|}{$\begin{array}{l}2018 \\
2020\end{array}$}} \\
\hline & & & & & & & & & & & \\
\hline & & & 468? & 325,200 & 2,897 & 3,358 & 331,464 & 236,444 & 191,020 & 3,006 & 2,499 \\
\hline องม & & & & 232,689 & 2,098 & 1,717 & & 236,448 & & & \\
\hline & & & & 175.4 & 0.8 & 1.6 & & 176.8 & & & \\
\hline 858 & & & & 189,045 & 1,680 & 1,111 & & & 191,820 & & \\
\hline & & & & 875. & 8.7 & 0.4 & & & 176.9 & & \\
\hline
\end{tabular}


PABSE C $C-23$

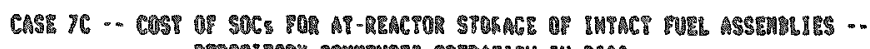

REP

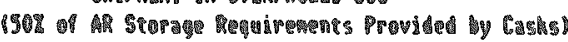

\begin{tabular}{|c|c|c|c|c|c|c|c|c|c|c|}
\hline \multirow[b]{3}{*}{ YEAR } & \multicolumn{10}{|c|}{ 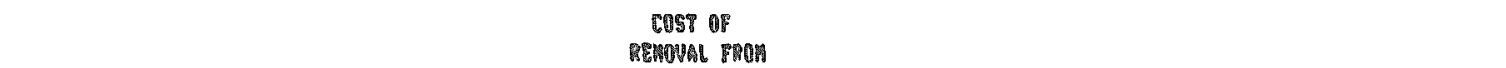 } \\
\hline & $\begin{array}{c}\text { TOTAL } \\
\text { ANACH: HUL } \\
\text { STORED M }\end{array}$ & MO. & $\begin{array}{l}\cos 2 \text { or } \\
\text { METAL }\end{array}$ & $\begin{array}{l}\text { COST OP } \\
\text { LOABIMC } \\
\text { S PHACE- } \\
\text { WEMT IN }\end{array}$ & 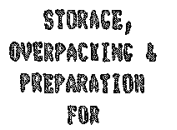 & & $\begin{array}{l}\text { POTAL COST } \\
\text { DIST. AP }\end{array}$ & $\begin{array}{l}\text { TOTAL COST } \\
\text { OISC.AT }\end{array}$ & 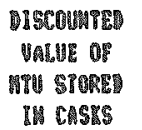 & 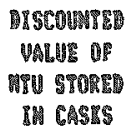 \\
\hline & 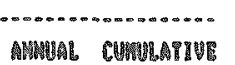 & 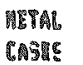 & $\begin{array}{l}\text { CA9PSS } \\
(1000)\end{array}$ & $\begin{array}{l}\text { STORAGE } \\
\text { PBOOU) }\end{array}$ & 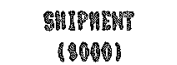 & $\begin{array}{l}\text { POYAL } \\
\text { POOOS }\end{array}$ & $\begin{array}{c}32101907 \\
800008\end{array}$ & 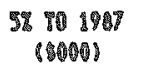 & 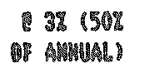 & $\begin{array}{l}5 \% \text { 150\% } \\
\text { OP MEMUAL }\end{array}$ \\
\hline
\end{tabular}

1997
1908
1908
1990
1991
1992
1993
1998
1995
1998
1997
1998
1998
2000
2001
2002
2003
2004
2005
2006
2007
2008
2009
2010
2011
2012
2013
2014
2015
2016
2017
2018
2019
2020

\begin{tabular}{|c|c|c|c|c|c|}
\hline 24 & 54 & 1 & 858 & 6 & \\
\hline 106 & 180 & $b$ & 5,736 & 37 & \\
\hline 163 & 323 & 8 & 7,030 & 56 & \\
\hline 126 & 449 & 7 & 5,355 & 43 & \\
\hline 238 & 897 & 13 & 0,793 & Bi & \\
\hline 280 & 973 & 15 & 19,070 & 93 & \\
\hline 258 & 1,231 & 14 & 10,206 & 17 & \\
\hline 423 & 1,859 & 23 & 16,583 & 193 & \\
\hline 365 & 2,024 & 20 & 18,240 & 124 & \\
\hline$\$ 54$ & 2,878 & 24 & 16,920 & 149 & \\
\hline 516 & 2,090 & 28 & 19,600 & 174 & \\
\hline $56 \%$ & 3,563 & 31 & 21,483 & 192 & \\
\hline 872 & 4,235 & 38 & 24,760 & 223 & \\
\hline 790 & 5,025 & 43 & 29,364 & 267 & \\
\hline$\$ 41$ & 5,869 & 45 & 30,510 & 279 & \\
\hline 927 & 8,796 & 50 & 33,650 & 310 & \\
\hline 1,015 & 7,811 & 55 & 38,795 & 341 & \\
\hline 806 & 0,717 & $4 P$ & 32,534 & 304 & \\
\hline 1,019 & 9,736 & 35 & 36,355 & 341 & \\
\hline 1,008 & 10,823 & 58 & 38,022 & 366 & \\
\hline 1,083 & 11,906 & 58 & 37,990 & 360 & \\
\hline 900 & 12,811 & 84 & 31,964 & 304 & \\
\hline 850 & 13,654 & S & 29,900 & 285 & \\
\hline 1,135 & 10,799 & 81 & 39,520 & 378 & \\
\hline \multirow[t]{15}{*}{750} & 15,549 & 41 & 26,527 & 251 & \\
\hline & & 1221 & & & 157 \\
\hline & & $(58)$ & & & 69 \\
\hline & & $\{103\}$ & & & 734 \\
\hline & & 11291 & & & 983 \\
\hline & & 11081 & & & 770 \\
\hline & & 11101 & & & 784 \\
\hline & & 11301 & & & 927 \\
\hline & & (1201 & & & 658 \\
\hline & & 1811 & & & 100 \\
\hline & & 838 & 367,648 & 5,190 & 5,98 \\
\hline & & & 368,786 & 3,312 & 2,541 \\
\hline & & & 879.8 & 0.7 & 1.5 \\
\hline & & & $270_{3}, 250$ & 2,510 & 1,457 \\
\hline & & & 774.3 & $B .7$ & 0.4 \\
\hline
\end{tabular}

\begin{tabular}{|c|c|c|c|c|}
\hline 962 & 98 & 862 & 12 & 12 \\
\hline 5,773 & 5,605 & 5,498 & 51 & 50 \\
\hline 7,094 & 0,687 & 6,134 & 78 & 74 \\
\hline 5,398 & 9,900 & 4,853 & 50 & 54 \\
\hline 9,844 & 18746 & 8,098 & 108 & 78 \\
\hline 11,163 & 9,629 & 8,747 & 123 & 112 \\
\hline 10,293 & 8,820 & 7,801 & 108 & 86 \\
\hline 16,726 & 13,600 & 11,887 & 174 & 152 \\
\hline 14,364 & 11,339 & 9,722 & 144 & 124 \\
\hline 17,069 & 13,082 & 11,003 & 174 & 146 \\
\hline 19,774 & 14,714 & 12,139 & 192 & 158 \\
\hline 22,675 & 15,859 & 12,673 & 208 & 166 \\
\hline 24,91 & 17,528 & 13,916 & 298 & 187 \\
\hline 29,636 & 20,181 & 15,716 & 269 & 209 \\
\hline 30,789 & 20,355 & 15,551 & 278 & 213 \\
\hline 33,960 & 21,796 & 16,335 & 298 & 223 \\
\hline 31,438 & 23,142 & 17,013 & 316 & 232 \\
\hline $3 z_{0} 80$ & 19,869 & 14,326 & 274 & 196 \\
\hline 36,698 & 21,555 & 15,208 & 299 & 212 \\
\hline 39,100 & 22,346 & 15,500 & 310 & 215 \\
\hline 38,350 & 21,233 & 14,454 & 300 & 204 \\
\hline 32,252 & 17,337 & 11,577 & 244 & 163 \\
\hline 30,185 & 15,754 & 10,318 & 222 & 145 \\
\hline 99,906 & 20,220 & 12,992 & 288 & 195 \\
\hline 26,781 & 13,175 & (1,304 & 184 & 118 \\
\hline 157 & 75 & 4 & & \\
\hline 698 & 324 & 197 & & \\
\hline 734 & 331 & 197 & & \\
\hline 983 & 421 & 248 & & \\
\hline 770 & 327 & 187 & & \\
\hline 784 & 323 & 101 & & \\
\hline 927 & 371 & 204 & & \\
\hline 858 & 392 & 180 & & \\
\hline 100 & 36 & 20 & & \\
\hline \multirow[t]{5}{*}{578,038} & 370,810 & 2192,226 & 4. & 3,747 \\
\hline & 370,610 & & & \\
\hline & $\$ 75.0$ & & & \\
\hline & & 212,226 & & \\
\hline & & 875.3 & & \\
\hline
\end{tabular}


TAแน $6-24$

CASE

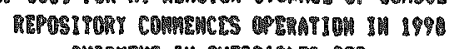

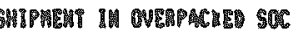

(50) of AR Storage Requipenents Provided by Cashs)

\begin{tabular}{|c|c|c|c|c|c|c|c|c|c|c|}
\hline \multirow[b]{3}{*}{ YreAR } & \multirow{3}{*}{\multicolumn{2}{|c|}{ 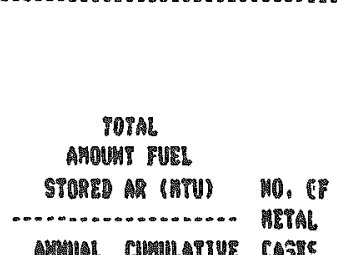 }} & \multirow{3}{*}{$\begin{array}{l}\text { CDI OP } \\
\text { METAL } \\
\text { CASIS }\end{array}$} & \multicolumn{3}{|c|}{$\begin{array}{c}\text { COSP OP } \\
\text { REMOVAL PROM }\end{array}$} & & & & \\
\hline & & & & $\begin{array}{l}\text { COST OB } \\
\text { LOMBIMG } \\
\text { 1 PLACE- } \\
\text { MEUT IM }\end{array}$ & 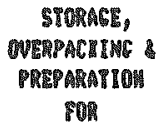 & & $\begin{array}{l}\text { TOTAL COST } \\
\text { DISC. }\end{array}$ & $\begin{array}{l}\text { TOYAL COST } \\
\text { UISC.AI }\end{array}$ & 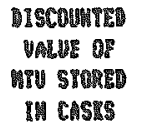 & 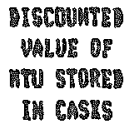 \\
\hline & & & & $\begin{array}{l}\text { STORAGE } \\
\text { (POOOO) }\end{array}$ & 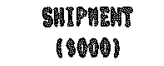 & $\begin{array}{l}\text { POPAL } \\
\text { POOOOS }\end{array}$ & $\begin{array}{c}38101987 \\
(0000)\end{array}$ & $\begin{array}{c}5 \% 101907 \\
\text { (1000) }\end{array}$ & 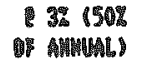 & 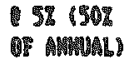 \\
\hline
\end{tabular}

\begin{tabular}{|c|c|c|c|c|c|c|c|c|c|c|c|}
\hline 1887 & 24 & 34 & 1 & 956 & 6 & & 962 & 182 & 862 & 12 & 12 \\
\hline 1883 & 108 & 180 & 4 & 3,824 & 25 & & 3,849 & 3,736 & 3,865 & 31 & 50 \\
\hline 1989 & 163 & 323 & 5 & 9,730 & 31 & & 4,811 & 4,535 & 4,363 & 77 & 74 \\
\hline 1990 & 126 & 449 & 4 & 3,12 & 25 & & 3,159 & 2,685 & 2,723 & 58 & 54 \\
\hline 1991 & 238 & 687 & 7 & 5,390 & 43 & & 5,123 & 4,827 & 9,970 & 108 & 91 \\
\hline 1992 & 286 & 973 & 10 & 7,5100 & 31 & & 7,621 & 6,574 & 5,972 & 123 & 112 \\
\hline 1993 & 258 & 1,231 & 0 & 5,968 & 9 & & 8,017 & 5,039 & 4,890 & 100 & 96 \\
\hline 1994 & 428 & 1,659 & 14 & 10,310 & 4 & & 10,804 & 3,459 & 7,394 & 174 & 152 \\
\hline 1995 & 365 & 2,024 & 12 & 0,746 & 74 & & 1,322 & 6,934 & 3,971 & 144 & 124 \\
\hline 1996 & 454 & 2,470 & 15 & 10,945 & 92 & & 10,077 & 1982 & 7,050 & 174 & 148 \\
\hline 1997 & 588 & 2,994 & 17 & 12,172 & 105 & & 12,277 & 0,135 & 7,597 & 192 & 158 \\
\hline 1998 & 189 & 3,163 & 5 & 3,560 & 31 & & 3,391 & 2,584 & 2,099 & 81 & 19 \\
\hline 1998 & 272 & 3,435 & 9 & 8,390 & 55 & & 8,485 & 1,521 & 3,594 & 95 & 76 \\
\hline 2000 & 390 & 3,125 & 12 & 8972 & 74 & & 8,546 & 5,119 & 4,532 & 133 & 103 \\
\hline 2001 & & & (1) & & & 7 & 9 & 5 & 4 & & \\
\hline 2002 & & & (29) & & & 205 & 205 & 192 & 99 & & \\
\hline 2003 & & & (84) & & & 492 & 452 & 282 & 207 & & \\
\hline 2001 & & & 1301 & & & 212 & 212 & 128 & 98 & & \\
\hline
\end{tabular}

TITAB

123

\begin{tabular}{|c|c|}
\hline 12,111 & 758 \\
\hline 13,832 & 102 \\
\hline$M 900$ & 3.1 \\
\hline 64,296 & 522 \\
\hline
\end{tabular}

877
597
404
402
6.3

93,744

74,900

63,220

1,500

1,306

TOTลL UISC ด3ะ

rotan arsc 05\%

UพIT COST/2

149.

4,

65,220
449.9 
Tตeน 6.25

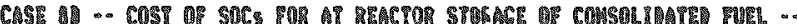

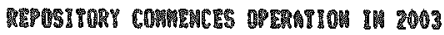

118 $4 / 19 \%$

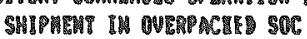

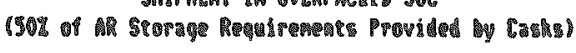

\begin{tabular}{|c|c|c|c|c|c|c|c|c|c|c|}
\hline \multirow{2}{*}{ YEAA } & \multirow{2}{*}{\multicolumn{2}{|c|}{ 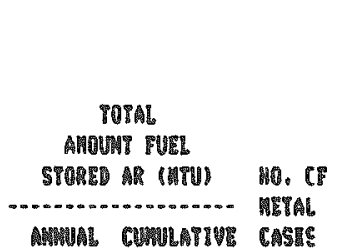 }} & \multirow{2}{*}{$\begin{array}{l}\text { COST OP } \\
\text { METAL } \\
\text { CASTE } \\
\text { (2000) }\end{array}$} & $\begin{array}{l}\text { COST OP } \\
\text { LOADIMG } \\
\text { PLACE- } \\
\text { FEMT IM } \\
\text { STORACE }\end{array}$ & 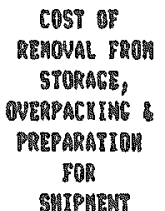 & & $\begin{array}{l}\text { TUPAL COST } \\
\text { DISC. AT } \\
\text { TR }\end{array}$ & $\begin{array}{l}\text { TOSAL COST } \\
\text { UISC APT }\end{array}$ & 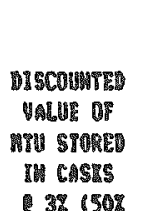 & 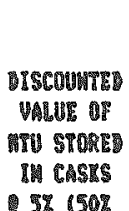 \\
\hline & & & & 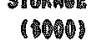 & ( & (18000) & $\begin{array}{l}\text { so } \\
\text { (1000) }\end{array}$ & 18000 & 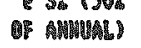 & บี \\
\hline
\end{tabular}

\begin{tabular}{|c|c|c|c|}
\hline 24 & 54 & 1 & 956 \\
\hline 108 & 180 & 4 & 3,820 \\
\hline 183 & 323 & 5 & 4,780 \\
\hline 126 & 949 & A & 3,128 \\
\hline 238 & 607 & $g$ & 5,390 \\
\hline 286 & 973 & 10 & 7,580 \\
\hline 258 & 1,231 & (6) & 5,988 \\
\hline 428 & 1,659 & 14 & 10,318 \\
\hline 345 & 2,024 & 12 & 8,740 \\
\hline 454 & 2,978 & 15 & 10,895 \\
\hline 516 & 2,990 & 17 & 12,172 \\
\hline 588 & 3,563 & 18 & 12,780 \\
\hline 672 & 1,235 & 22 & 15,888 \\
\hline 790 & 5,025 & 24 & 10,178 \\
\hline 84 & 5,868 & 27 & 18,718 \\
\hline 927 & 6,798 & 31 & 21,359 \\
\hline 615 & 7,811 & 20 & 13,700 \\
\hline 506 & 7,917 & 17 & 11,811 \\
\hline 619 & 8,536 & 20 & 13,820 \\
\hline \multirow[t]{8}{*}{187} & 3,723 & 8 & 4,070 \\
\hline & & $(83)$ & \\
\hline & & 1551 & \\
\hline & & 1571 & \\
\hline & & 1981 & \\
\hline & & (4A4) & \\
\hline & & 1581 & \\
\hline & & 31 & \\
\hline
\end{tabular}

8
25
31
29
43
41
48
86
11
92
105
111
135
160
166
111
123
105
123
37

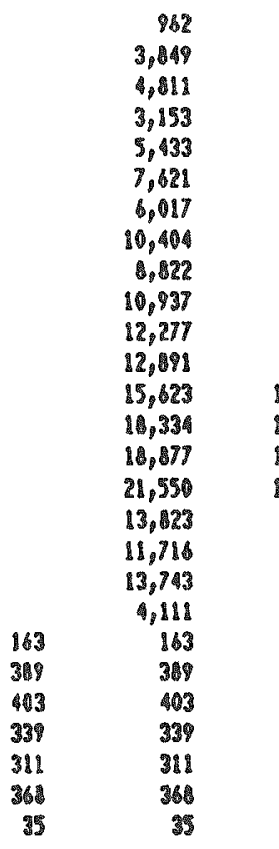

\begin{tabular}{|c|c|}
\hline 982 & 962 \\
\hline 3,738 & 3,665 \\
\hline 8,535 & 8,363 \\
\hline 2,895 & 2,723 \\
\hline 6827 & 4,470 \\
\hline 8,574 & 5,972 \\
\hline 5,038 & 1490 \\
\hline 6,459 & 8,394 \\
\hline 8,964 & 5,971 \\
\hline 5382 & 7,050 \\
\hline 8135 & 7,537 \\
\hline 9,312 & $7_{1} 537$ \\
\hline 10.958 & 8,700 \\
\hline 12,484 & 9,723 \\
\hline 12,480 & 8,534 \\
\hline 13,892 & 10,388 \\
\hline 8,814 & 8392 \\
\hline 7,088 & 5,811 \\
\hline 8073 & 5,710 \\
\hline 2,394 & 1,627 \\
\hline 9 & $\$ 1$ \\
\hline 209 & 140 \\
\hline 210 & 13 \\
\hline 172 & 110 \\
\hline 153 & 81 \\
\hline 176 & 109 \\
\hline 10 & ר \\
\hline
\end{tabular}

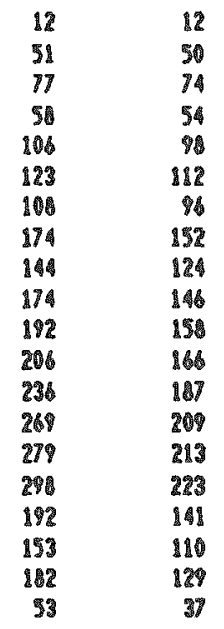

2,00

208960

\section{7,712}

119,902

tist

$\begin{array}{rrr}185,648 & 1,299 & 1,026 \\ 017.1 & 8.4 & 8.3 \\ 118,238 & 1,002 & 864\end{array}$

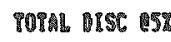

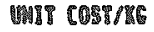

$\begin{array}{rrr}118,238 & 1,002 & 864 \\ 147.4 & 8.4 & 8.3\end{array}$

119,902


TAMLอ C- 26

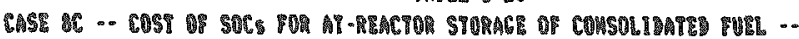

REPOSI TORY COMAEMCES OPERATIOM IW 2004

11/ 4/1997

1502 of AR Storege Re?

\begin{tabular}{|c|c|c|c|c|c|c|c|c|c|c|c|}
\hline YreAR & $\begin{array}{r}\text { To } \\
\text { AMOU\% } \\
\text { SPORES }\end{array}$ & 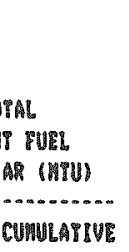 & $\begin{array}{l}\text { MO. OP } \\
\text { MEPAL } \\
\text { CASTS }\end{array}$ & $\begin{array}{l}\text { COST OP } \\
\text { MEPAL } \\
\text { CASIS } \\
\text { (POOO) }\end{array}$ & $\begin{array}{l}\text { COST OP } \\
\text { LOABLWC } \\
\text { DLACE- } \\
\text { MEDI IN } \\
\text { STORAGE } \\
\text { (BOOOO) }\end{array}$ & 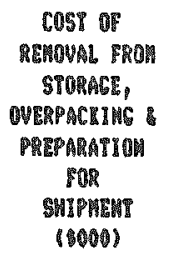 & $\begin{array}{l}\text { TOYAL } \\
\text { (1000) }\end{array}$ & $\begin{array}{c}\text { TOTAL COST } \\
\text { IISC. AP } \\
32 \text { Y0 1987 } \\
\text { (8000) }\end{array}$ & $\begin{array}{c}\text { POTAL COST } \\
\text { MSC.AP } \\
\text { \$\% PO 19M7 } \\
\text { 18060) }\end{array}$ & 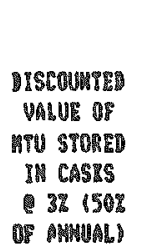 & $\begin{array}{l}\text { ISTCUWFE } \\
\text { VALUE OP } \\
\text { MTU STORE } \\
\text { IN CASYS } \\
5 \% 150 \% \\
\text { OF MAWUAL }\end{array}$ \\
\hline 1987 & 24 & 34 & 1 & 958 & 6 & 8 & 962 & 982 & $\$ 82$ & 12 & 12 \\
\hline 1988 & 106 & 160 & 4 & 9,824 & 25 & & 3,840 & 3,736 & 3,685 & 51 & 50 \\
\hline 1989 & 163 & 323 & 5 & 9,700 & 31 & & 7,811 & 835 & 1,383 & $n$ & 74 \\
\hline 1890 & 126 & 448 & 4 & 3,128 & 25 & & 3,153 & 2,885 & 2,723 & 58 & 54 \\
\hline 1991 & 238 & 687 & i & 5,390 & 43 & & 5,433 & 4,827 & 4.970 & 106 & 98 \\
\hline 1012 & 206 & 973 & 10 & 7,580 & 61 & & $7 \div 621$ & 6,374 & 5,972 & 123 & 112 \\
\hline 1993 & 258 & 1,231 & 8 & 5,960 & 49 & & 6,017 & $\$, 039$ & 4,490 & 100 & 96 \\
\hline 1894 & 426 & 1,658 & 14 & 10,310 & $\$$ & & 10,404 & 6,459 & 7,394 & 174 & 152 \\
\hline 1985 & 365 & 2,024 & 12 & 1,748 & 74 & & 8,822 & 8,984 & 5,971 & 144 & 124 \\
\hline 1996 & 454 & 2,478 & 15 & 10,845 & 92 & & 10,937 & 8,382 & 7,050 & 174 & 146 \\
\hline 1977 & 516 & 2,994 & 17 & 12,172 & 105 & & 12,277 & 8,135 & 7,537 & 192 & 154 \\
\hline 1998 & 569 & 3,563 & 18 & 12,790 & III & & 12,891 & 8,312 & 7,537 & 206 & 186 \\
\hline 1999 & 872 & 4,235 & 22 & 15,96 & 135 & & 15,623 & 10,958 & 8700 & 236 & 187 \\
\hline 2000 & 790 & 5,025 & 26 & 10,184 & 180 & & 10,334 & 12,484 & 9,723 & 289 & 209 \\
\hline 2001 & 844 & 5,869 & 27 & 18,711 & 186 & & 10,897 & 12,480 & 9,534 & 279 & 213 \\
\hline 2002 & 927 & 6,796 & 31 & 21,359 & 191 & & 21,550 & 13,892 & 10,386 & 210 & 223 \\
\hline 2003 & 1,015 & 8,811 & 32 & 21,888 & 197 & & 22,005 & 13,782 & 10,117 & 316 & 232 \\
\hline 2004 & 806 & 8,717 & 30 & 20,400 & 184 & & 20,584 & 12,454 & 8,981 & 274 & 190 \\
\hline 2005 & 1,019 & 8,736 & 33 & 22,341 & 203 & & 22,594 & 13,242 & 8,367 & 299 & 212 \\
\hline 2006 & 1,087 & 10,123 & 35 & 23,555 & 215 & & 23,770 & 13,556 & 9,407 & 310 & 215 \\
\hline 2007 & 1,003 & 11,908 & 35 & 23,450 & 215 & & 23,865 & 13,103 & 6,918 & 300 & 204 \\
\hline 2000 & 900 & 12,814 & 20 & 19,372 & 178 & & 19,550 & 10,509 & 7,017 & 244 & 183 \\
\hline 2009 & 850 & 13,864 & 28 & 18,548 & 172 & & 18,820 & 9,822 & 8,434 & 222 & 145 \\
\hline 2010 & 1135 & 14,799 & 37 & 24,531 & 227 & & 24,750 & 12,545 & 6,061 & 280 & 165 \\
\hline 2011 & 750 & 15,549 & 28 & 15,080 & 14 & & 16,012 & 7,877 & 4,985 & 184 & IIS \\
\hline 2012 & & & (13) & & & 92 & 92 & 91 & 27 & & \\
\hline 2013 & & & 1591 & & & 417 & 417 & 193 & 117 & & \\
\hline 20114 & & & 1621 & & & A36 & 438 & 197 & 117 & & \\
\hline 2015 & & & (81) & & & 573 & 573 & 250 & 146 & & \\
\hline 2016 & & & 1651 & & & 460 & 460 & 185 & 112 & & \\
\hline 2017 & & & (Bn) & & & 974 & 474 & 195 & 110 & & \\
\hline 2018 & & & (78) & & & 551 & 551 & 221 & 122 & & \\
\hline 2019 & & & $(72)$ & & & 508 & 509 & 198 & 107 & & \\
\hline 2020 & & & 191 & & & 84 & 64 & 34 & 13 & & \\
\hline TOTAL & & & 504 & 350,250 & 3,099 & 3,57 & 356,926 & 220,951 & 174,595 & 4,843 & 3,747 \\
\hline TOPAL IISC Q38 & & & & 225,461 & 1,975 & 1,517 & & 228,954 & & & \\
\hline טHIT cosiา & & & & $\$ 45.6$ & 1.4 & 8.3 & & $\$ 46.3$ & & & \\
\hline TORAL UISC ISE & & & & 172,227 & 1,998 & 870 & & & 174,585 & & \\
\hline UAII COST/RG & & & & $\$ 46,0$ & 8.4 & 1.2 & & & 854.8 & & \\
\hline
\end{tabular}


PAMLE C.27

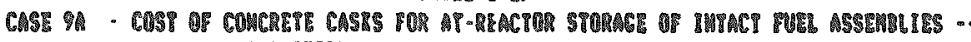
REPOSITOMY COAMEMCES OPERATIOA IN 1896

sog of ar Storage Requiraments Fovided by Cashs!

\begin{tabular}{|c|c|c|c|c|c|c|c|c|c|}
\hline 19.0 0 & $\begin{array}{l}\text { COST OP } \\
\text { COMCRETR } \\
\text { CaeYg }\end{array}$ & 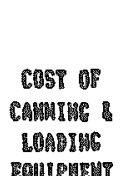 & 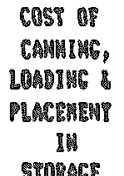 & 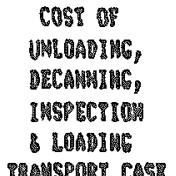 & & $\begin{array}{l}\text { TOTAL COST } \\
\text { DISE. AI }\end{array}$ & $\begin{array}{l}\text { TOYAL COST } \\
\text { UISC. }\end{array}$ & 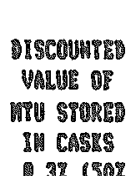 & 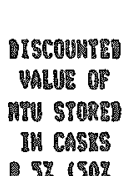 \\
\hline 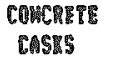 & $\begin{array}{l}\text { CA5Es } \\
\text { 10000 }\end{array}$ & $\begin{array}{l}\text { Fou1PWEHT } \\
\text { (1000) }\end{array}$ & 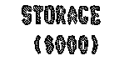 & $\begin{array}{l}\text { PRANSPORT CAST } \\
\text { (8000) }\end{array}$ & $\begin{array}{l}\text { POTAL } \\
\text { (2000) }\end{array}$ & $\begin{array}{l}32901987 \\
160009\end{array}$ & $\begin{array}{c}\text { 5\% 10 } 1907 \\
\text { (6000) }\end{array}$ & 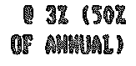 & 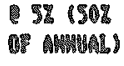 \\
\hline
\end{tabular}

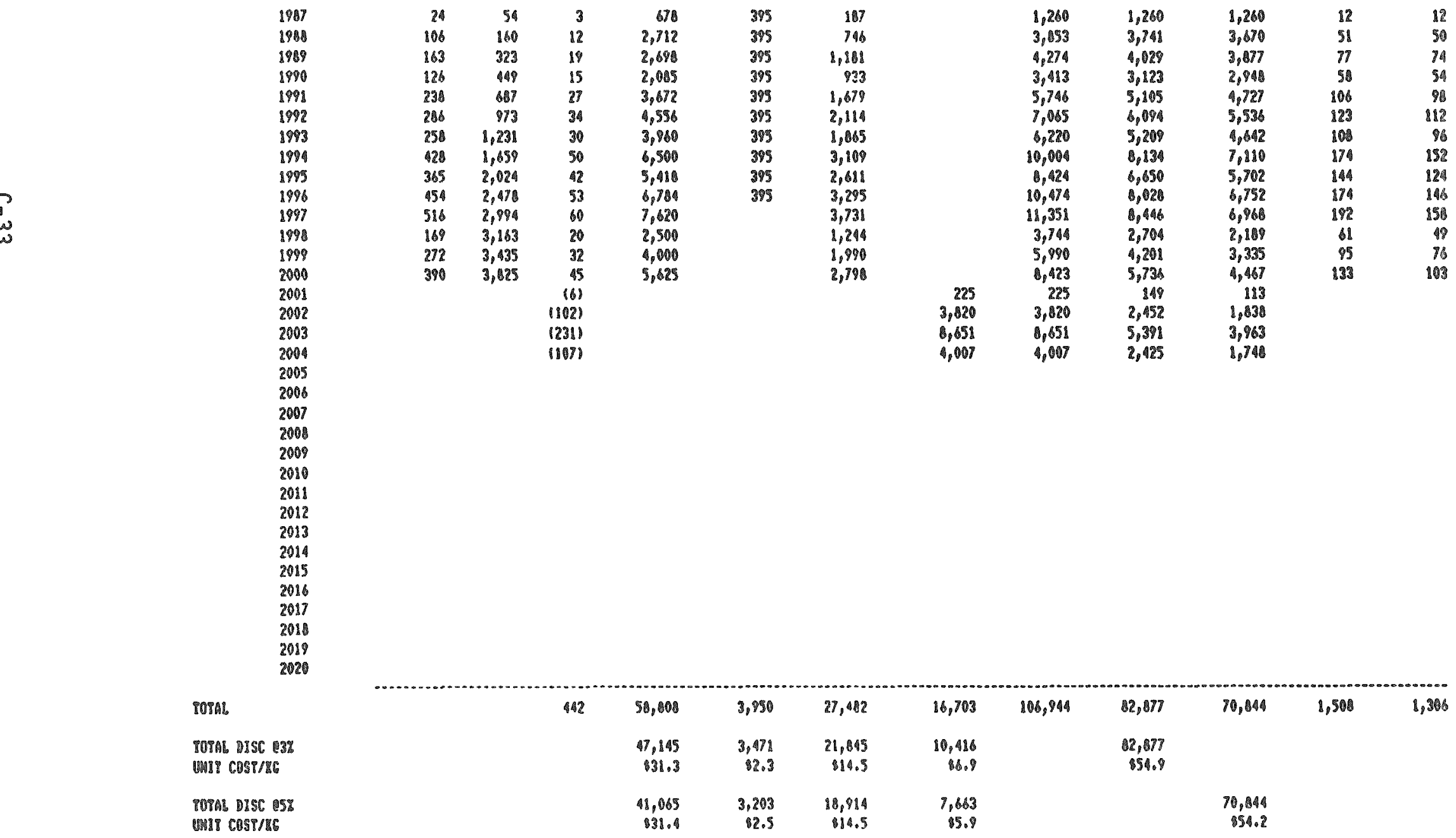


TABL [- 20

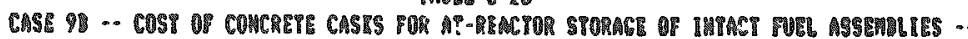

REPQSITER COMHENCES OPRRATHOA IN 2003

$150 \%$ of at storane Remairenests Provided by Cashal

\begin{tabular}{|c|c|c|c|c|c|c|c|c|c|c|c|}
\hline $\mathrm{EA}$ & 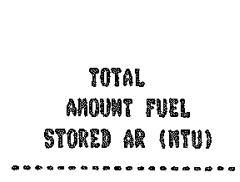 & $\begin{array}{c}\text { mo. of } \\
\text { consorpte }\end{array}$ & $\begin{array}{l}\text { COST OP } \\
\text { COMCEETE } \\
\text { CASYS }\end{array}$ & 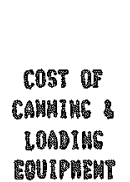 & 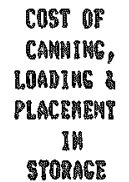 & 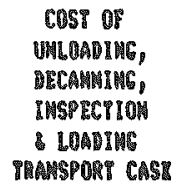 & ToTAL & $\begin{array}{c}\text { I0TAL cost } \\
\text { IISC. AT } \\
3 \% \text { T0 } 107\end{array}$ & $\begin{array}{c}\text { TOTAL COST } \\
\text { ISC.AP } \\
5 \% \text { T0 } 1987\end{array}$ & 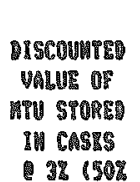 & 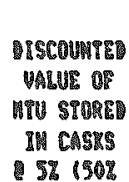 \\
\hline & 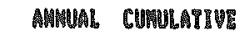 & CASES & (5000) & $(1000)$ & 100001 & 100009 & (\$อง) & 100001 & 100001 & 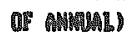 & (7) \\
\hline
\end{tabular}

1987
1986
1999
1990
1991
1992
1999
1991
1895
1996
1997
1999
1999
2000
2001
2002
2003
2004
2005
2006
2007
2008
2009
2010
2011
2012
2013
2011
2015
2016
2017
2018
2019
2020

$\begin{array}{rr}21 & 54 \\ 108 & 160 \\ 163 & 323 \\ 128 & 469 \\ 238 & 607 \\ 288 & 973 \\ 258 & 1,291 \\ 128 & 1,659 \\ 365 & 2,024 \\ 454 & 2,470 \\ 516 & 2,994 \\ 569 & 3,563 \\ 872 & 4,235 \\ 790 & 5,025 \\ 944 & 5,869 \\ 927 & 6,796 \\ 615 & 7,411 \\ 508 & 7,917 \\ 619 & 6,536 \\ 107 & 0,723\end{array}$

3
12

778
2,712
2,698

2,698

3,078

4,556

3,960

6,500

5,410

6,784

7,620

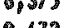

9,672

11,956

13,176

9,712

7,139

2,140

395
395
395

395
395
395
395

107
798

1,101

1,679

2,114

1,365

3,108

2,611

3,295
3,731

3,731

4166

5. 850

8,093

8,093
8,715

4,477

3,665

1,977

\begin{tabular}{|c|c|c|c|}
\hline & 1,280 & 1,260 & 1,280 \\
\hline & 3,853 & 3,741 & 3,070 \\
\hline & 1,278 & 4,029 & 3,877 \\
\hline & 3,413 & 3,123 & $2, P 48$ \\
\hline & 9,746 & 5,105 & 4,727 \\
\hline & 7,065 & 8,094 & 5,598 \\
\hline & 8,220 & 5,209 & 4,642 \\
\hline & 10,000 & 9,134 & 7,110 \\
\hline & 8,24 & 6,650 & 5,702 \\
\hline & 10,877 & 8026 & 4.752 \\
\hline & 12,141 & 8,094 & 7,453 \\
\hline & 13,331 & 8,630 & 7,794 \\
\hline & 15,312 & 10,799 & 0,528 \\
\hline & 17,288 & 12,139 & 0,154 \\
\hline & 16,839 & 12,455 & 0,515 \\
\hline & 19,091 & 12,767 & 9,586 \\
\hline & 13,109 & 0,219 & 0,042 \\
\hline & 10,807 & 4,539 & 4,715 \\
\hline & 13,117 & 8,705 & 5,950 \\
\hline & 4,000 & $2,2 M$ & 1,504 \\
\hline 3,146 & 3,146 & 1,742 & 1,14 \\
\hline 7,378 & 7,378 & 3,960 & 2,648 \\
\hline 7,640 & 7,640 & 3,967 & 2,612 \\
\hline 6,367 & 8,387 & 3,226 & 2,073 \\
\hline 5,917 & $\$, 917$ & 2,911 & 1,835 \\
\hline 7,041 & 7,018 & 3,363 & 2,079 \\
\hline 599 & 599 & 276 & 109 \\
\hline
\end{tabular}

$\begin{array}{rr}12 & 12 \\ 51 & 50 \\ 77 & 71 \\ 58 & 54 \\ 106 & 91 \\ 129 & 112 \\ 106 & 96 \\ 174 & 152 \\ 114 & 124 \\ 184 & 114 \\ 192 & 150 \\ 206 & 186 \\ 236 & 187 \\ 289 & 209 \\ 279 & 213 \\ 291 & 223 \\ 192 & 141 \\ 153 & 110 \\ 122 & 129 \\ 53 & 37\end{array}$

T0นิ

1013

120,309
91,923

7,800
0,243

62,995

38,0

237,282

162,959

128,927

3,006

2, 493

U⿻丷木⿴囗十 COST/R6

81,833
829.8

44,710
114.5

$\$ 6.3$

2,359

$74,793 \quad 5,407 \quad 36,129 \quad 12,001$

TOTAL DISC อง

$\$ 30.0$

114.

85.1

126,928

551.7 
TALLE C-29

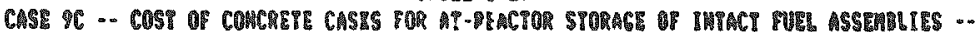

REPOSITORY COWHEWCES OPRRATION IM 2000

$11 / 3 / 1987$

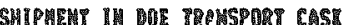

150\% of AR Stopage Reąui renents Provided by Caghs)

\begin{tabular}{|c|c|c|c|c|c|c|c|c|c|c|c|}
\hline & $\begin{array}{c}\text { TOTAL } \\
\text { AMOUM YUEL } \\
\text { SPORE M }\end{array}$ & MO. OP & $\begin{array}{l}\text { COST OP } \\
\text { COMCRETE }\end{array}$ & $\begin{array}{l}\text { COST OP } \\
\text { CAMYING } \\
\text { LOADIMG }\end{array}$ & $\begin{array}{l}\text { COST OF } \\
\text { CAMHING, } \\
\text { LOADIMC E } \\
\text { PLACEMENT } \\
\text { II }\end{array}$ & 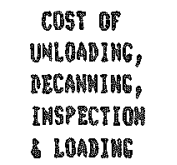 & & $\begin{array}{l}\text { TOTAL COST } \\
\text { DISC, AI }\end{array}$ & $\begin{array}{l}\text { DTAL COST } \\
\text { DISC.AT }\end{array}$ & 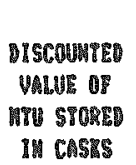 & 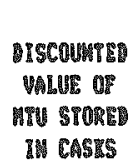 \\
\hline YRAR & 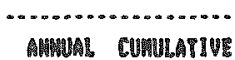 & $\begin{array}{l}\text { COMCMLTE } \\
\text { CASบS }\end{array}$ & $\begin{array}{l}\text { Cก:Ts } \\
\text { (s000) }\end{array}$ & $\begin{array}{l}\text { EaviphenT } \\
\text { (9000) }\end{array}$ & $\begin{array}{l}\text { STORAGE } \\
\text { (9000) }\end{array}$ & $\begin{array}{l}\text { PRASPPORT CASE } \\
\text { (15000 }\end{array}$ & $\begin{array}{l}\text { TOTAL } \\
\text { (10000) }\end{array}$ & $\begin{array}{c}38101917 \\
180001\end{array}$ & $\begin{array}{l}58 \text { 10 } 19797 \\
(9500)\end{array}$ & $\begin{array}{l}\text { 3ิ } 150 \% \\
\text { of ANMEAL) }\end{array}$ & $\begin{array}{l}\text { 5\% (502 } \\
\text { Of AME:AL) }\end{array}$ \\
\hline
\end{tabular}

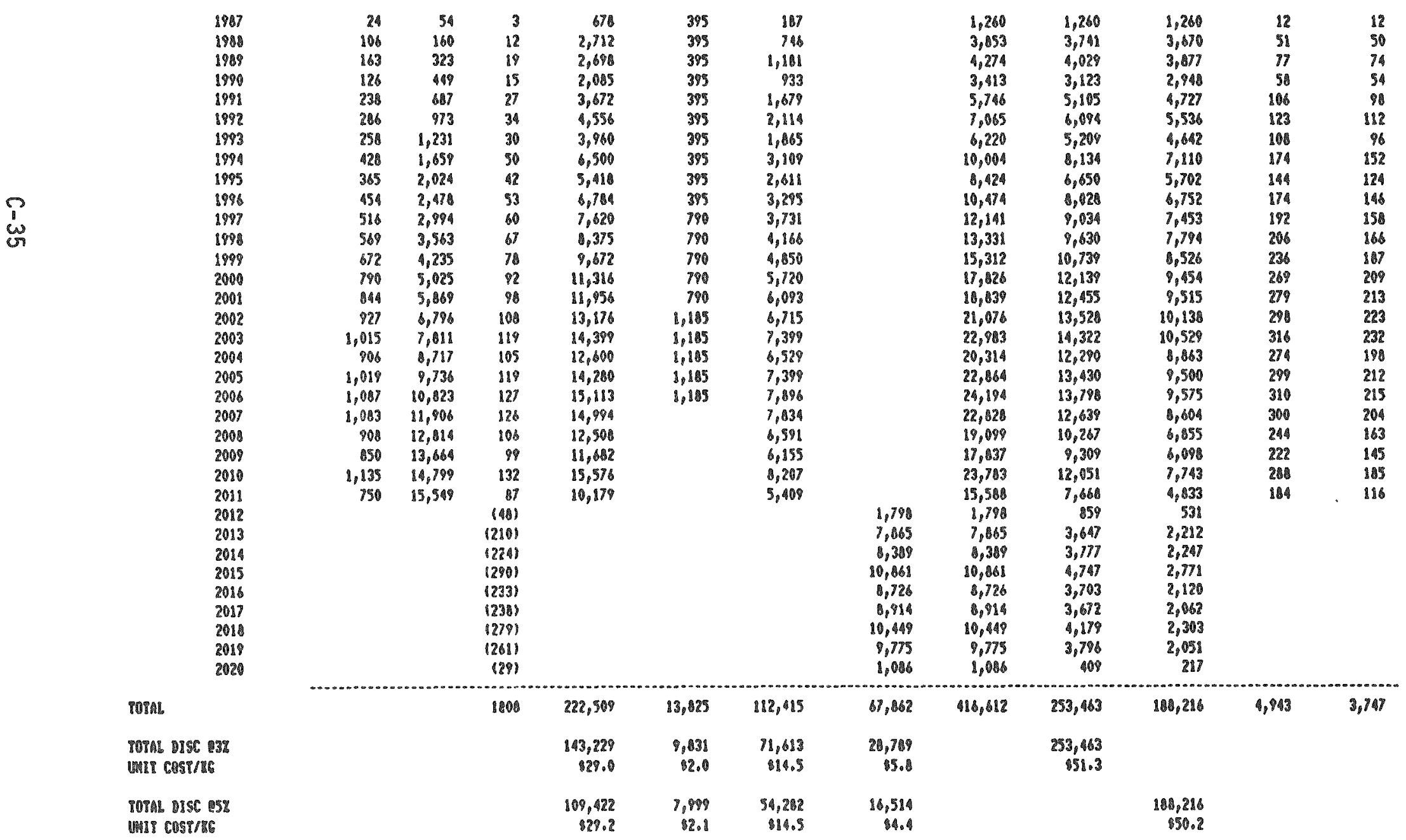




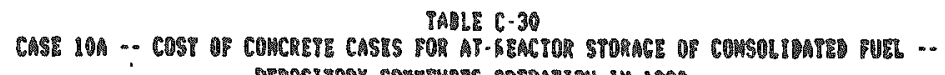

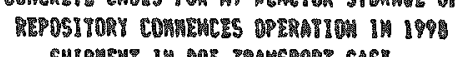

11) $3 / 1987$

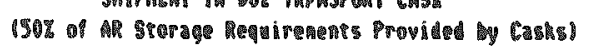

\begin{tabular}{|c|c|c|c|c|c|c|c|c|c|c|c|c|}
\hline YEAR & 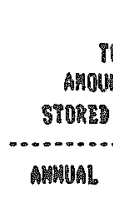 & 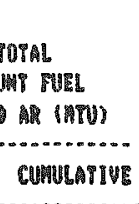 & 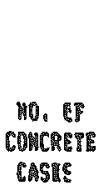 & 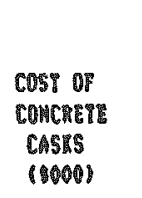 & 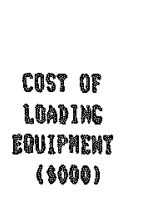 & $\begin{array}{c}\text { COST OF } \\
\text { LOALIMC \& } \\
\text { PLACEMEMT } \\
\text { 11 } \\
\text { STORACE } \\
\text { (1000) }\end{array}$ & 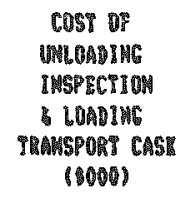 & $\begin{array}{l}\text { YOPAL } \\
\text { (BOQO) }\end{array}$ & 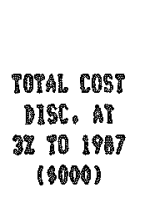 & 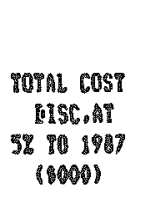 & 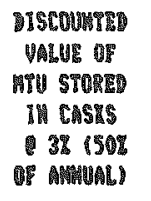 & 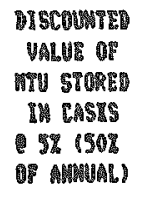 \\
\hline 1987 & 2 & 31 & 1 & 226 & 250 & 23 & & 199 & จุร & 498 & 12 & 12 \\
\hline 1988 & 10 & 160 & 8 & 1,800 & 250 & 185 & & 2,243 & 2,178 & 2,136 & 51 & 58 \\
\hline 1989 & 16. & 323 & 11 & 2,400 & 250 & 255 & & 2,991 & 2,018 & 2,713 & 77 & 74 \\
\hline 1998 & 12 & 449 & 9 & 1,270 & 250 & 200 & & 1,736 & 1,588 & 1,500 & 58 & 58 \\
\hline 1991 & 23 & 687 & 1) & 2,300 & 250 & 393 & & 3,023 & $2_{B} 608$ & 2,487 & 108 & 90 \\
\hline 1992 & 28 & 973 & 20 & 2,720 & 250 & 463 & & 3,433 & 2,961 & 2,690 & 123 & 112 \\
\hline 1999 & 25 & 1,231 & 18 & 2,430 & 250 & 417 & & 3,097 & 2,593 & 2,311 & 108 & 96 \\
\hline 1999 & 42 & 1,859 & 30 & 3,990 & 250 & 694 & & 4,934 & 1,012 & 3,507 & 174 & 152 \\
\hline 1995 & 36 & 2,024 & 24 & 3,432 & 250 & BC2 & & 4,231 & 3,382 & 2,899 & 144 & 124 \\
\hline 1896 & 45 & 2,478 & 31 & 4,061 & 250 & 717 & & 5,021 & 3,854 & 3,241 & 174 & 146 \\
\hline 1897 & 51 & 2,994 & y & 4,120 & & 858 & & 5,606 & 4,218 & 3,479 & 192 & 158 \\
\hline 1991 & 16 & 3,1103 & 12 & 1,540 & & 278 & & 1,820 & 1,318 & 1,067 & 11 & 49 \\
\hline 1999 & 27 & 3,435 & 18 & 2,451 & & 400 & & 2,891 & 2,027 & 1,818 & 95 & 78 \\
\hline 2000 & 39 & 3,225 & 27 & 3,456 & & 825 & & 4,001 & 2,779 & 2,184 & 133 & 103 \\
\hline 2001 & & & (4) & & & & 111 & 114 & 78 & 38 & & \\
\hline 2002 & & & (61) & & & & 1,742 & $1,7 / 42$ & 1,118 & 38 & & \\
\hline 2003 & & & 11401 & & & & 3,897 & 3,097 & 2,011 & 1,831 & & \\
\hline 20004 & & & 8641 & & & & 1,027 & 1,827 & 1,108 & 797 & & \\
\hline 2005 & & & & & & & & & & & & \\
\hline 2006 & & & & & & & & & & & & \\
\hline 2007 & & & & & & & & & & & & \\
\hline 2008 & & & & & & & & & & & & \\
\hline 2009 & & & & & & & & & & & & \\
\hline 2010 & & & & & & & & & & & & \\
\hline 2011 & & & & & & & & & & & & \\
\hline 2012 & & & & & & & & & & & & \\
\hline 2013 & & & & & & & & & & & & \\
\hline 2014 & & & & & & & & & & & & \\
\hline 2015 & & & & & & & & & & & & \\
\hline 2016 & & & & & & & & & & & & \\
\hline 2017 & & & & & & & & & & & & \\
\hline 2018 & & & & & & & & & & & & \\
\hline 2018 & & & & & & & & & & & & \\
\hline 2020 & & & & & & & & & & & & \\
\hline POPAL & & & 286 & 37,078 & 2,500 & 6,155 & 7,600 & 53,412 & 41,704 & 35,026 & 1,500 & 1,306 \\
\hline TOTAL UISC E3\% & & & & 29,826 & 2,197 & 1,192 & $\$, 790$ & & 11,704 & & & \\
\hline UNIY cosm/re & & & & 10.0 & 81.5 & 33.2 & 83.2 & & 227.6 & & & \\
\hline TOTAL BISC Q5\% & & & & 26,041 & 2,027 & 4,298 & 3,524 & & & 35,826 & & \\
\hline UMIT COST/RC & & & & $\$ 19.9$ & $\$ 1.8$ & $\$ 3.2$ & 82.7 & & & 877.4 & & \\
\hline
\end{tabular}


TABLE C-3!

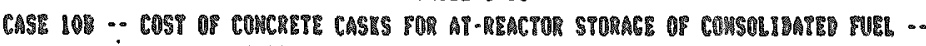

REPOSITORY COMAEMESE OPERATTOM III 2003

SMIPATE IV OOE TAAMSPORT CASE

1502 of Am Storage Requirenents Proviched by Cashs?

\begin{tabular}{|c|c|c|c|c|c|c|c|c|c|c|c|c|}
\hline YEAG & 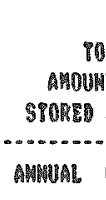 & 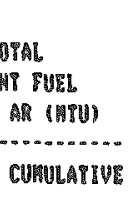 & $\begin{array}{l}\text { No. OF } \\
\text { COMCRET } \\
\text { CASES }\end{array}$ & $\begin{array}{l}\text { COST OF } \\
\text { COHCRET } \\
\text { CASTS } \\
\text { (1900) }\end{array}$ & 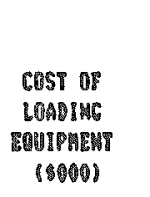 & $\begin{array}{c}\text { COSP OR } \\
\text { LOABIUG } \\
\text { PLACEMEAT } \\
\text { IN } \\
\text { STORAGE } \\
\text { I }\end{array}$ & 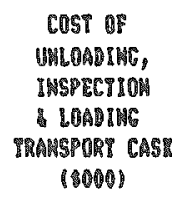 & $\begin{array}{l}\text { POTAL } \\
\text { PO000) }\end{array}$ & 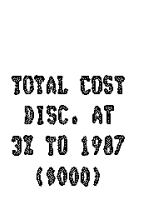 & 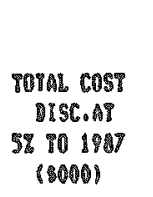 & 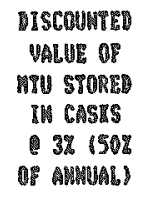 & 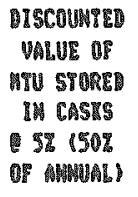 \\
\hline 1807 & 24 & 58 & 1 & 224 & 250 & 23 & & 499 & 499 & 499 & 12 & 12 \\
\hline 1988 & 106 & 180 & 0 & $\$ 800$ & 250 & 185 & & 2,243 & 2,178 & 2,136 & 51 & 50 \\
\hline 1989 & 183 & 323 & 11 & 2,486 & 250 & 255 & & 2,891 & 2,819 & 2,713 & 77 & 74 \\
\hline 1990 & 126 & 948 & 9 & 1,271 & 250 & 208 & & 1,736 & 1,589 & 1,500 & 58 & 54 \\
\hline 1991 & 238 & 687 & 17 & 2,380 & 250 & 393 & & 3,023 & 2,634 & 2,487 & 106 & 9 \\
\hline 1992 & 286 & 973 & 20 & 2,720 & 250 & 463 & & 3,433 & 2981 & 2,690 & 123 & 112 \\
\hline 1993 & 250 & 1,231 & 18 & 2,430 & 250 & 417 & & 3,097 & 2,593 & 2,311 & 108 & 96 \\
\hline 1994 & 428 & 1,658 & 30 & 3,990 & 250 & 694 & & 4,834 & 4,012 & 3,507 & 174 & 152 \\
\hline 1895 & 385 & 2,024 & 26 & 3,432 & 250 & 802 & & 4,284 & 3,382 & 2,899 & 134 & 124 \\
\hline 1996 & 454 & 2,476 & 31 & 9,061 & 250 & 717 & & 5,028 & 3,854 & 3,241 & 174 & IAS \\
\hline 1897 & 516 & 2,994 & 37 & 4,810 & 500 & 35.4 & & 6,166 & 4,588 & 3,785 & 192 & 150 \\
\hline 1890 & 569 & 3,563 & 40 & 5,160 & 500 & $92 \%$ & & 1,586 & 9758 & 3,050 & 206 & 166 \\
\hline 1899 & 672 & 4,235 & 47 & 6,016 & 500 & 1,008 & & 7,604 & 5,333 & 4,234 & 238 & 107 \\
\hline 2090 & 790 & 5,025 & 55 & 6,935 & 500 & 1,273 & & 8,750 & 5,984 & 4644 & 268 & $20 \%$ \\
\hline 2001 & 344 & 5,969 & 20 & 7,500 & 500 & 1,388 & & 9,387 & 8,207 & 4,742 & $27 \%$ & 213 \\
\hline 2002 & 927 & 8,796 & 65 & 1,0000 & & 1,504 & & 9,564 & 8,139 & 4,600 & 298 & 223 \\
\hline 2003 & $\$ 15$ & 7,411 & 43 & 5,332 & & 998 & & 6,327 & 3,943 & 2,898 & 192 & 141 \\
\hline 2004 & 506 & 7.917 & 35 & 4,305 & & 810 & & 5,115 & 3,095 & 2,232 & 153 & 110 \\
\hline 2005 & 819 & 8,536 & 40 & 5,412 & & 1,018 & & 8,430 & 3,777 & 2,672 & 182 & 129 \\
\hline 2006 & 187 & 8,723 & 13 & 1,599 & & 301 & & 8,900 & 1,083 & 752 & 53 & 37 \\
\hline 2007 & & & 8501 & & & & 1,428 & 1,428 & 790 & 538 & & \\
\hline 2008 & & & (1119) & & & & 3,398 & 3,398 & 1,026 & 1,220 & & \\
\hline 2009 & & & (123) & & & & 3,512 & 3,512 & 1,833 & 1,201 & & \\
\hline 2010 & & & (1003) & & & & 2,941 & 2,941 & 1,480 & 957 & & \\
\hline 2018 & & & (85) & & & & 2,712 & 2,712 & 1,334 & 841 & & \\
\hline 2012 & & & (1113) & & & & 3ి,226 & 3,226 & 8,541 & 953 & & \\
\hline 2013 & & & 8101 & & & & 288 & 288 & 192 & 80 & & \\
\hline 2014 & & & & & & & & & & & & \\
\hline 2015 & & & & & & & & & & & & \\
\hline 2016 & & & & & & & & & & & & \\
\hline 2017 & & & & & & & & & & & & \\
\hline 2018 & & & & & & & & & & & & \\
\hline 2018 & & & & & & & & & & & & \\
\hline 2020 & & & & & & & & & & & & \\
\hline TDPA & & & 610 & 79,990 & 5,000 & 19,115 & 17,502 & 186,607 & 20,408 & 64,183 & 3,086 & 2,493 \\
\hline 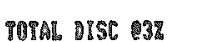 & & & & 57,898 & 3,952 & 10,018 & 0,897 & & 100,108 & & & \\
\hline UมIP COST/RG & & & & 110.6 & 01.3 & $\$ 3.2$ & 82.9 & & 826.1 & & & \\
\hline PพTAL DISE อ5ร & & & & 98,877 & 3,422 & 0,094 & 5,790 & & & 64,183 & & \\
\hline OMIT CUST/NG & & & & 810.8 & 11.0 & 13.2 & 82.3 & & & 825.7 & & \\
\hline
\end{tabular}


PABLE [-3?

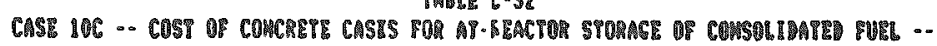

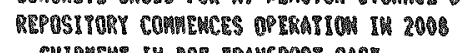

150\% of ar Stopage Requirements Providel by Cashs)

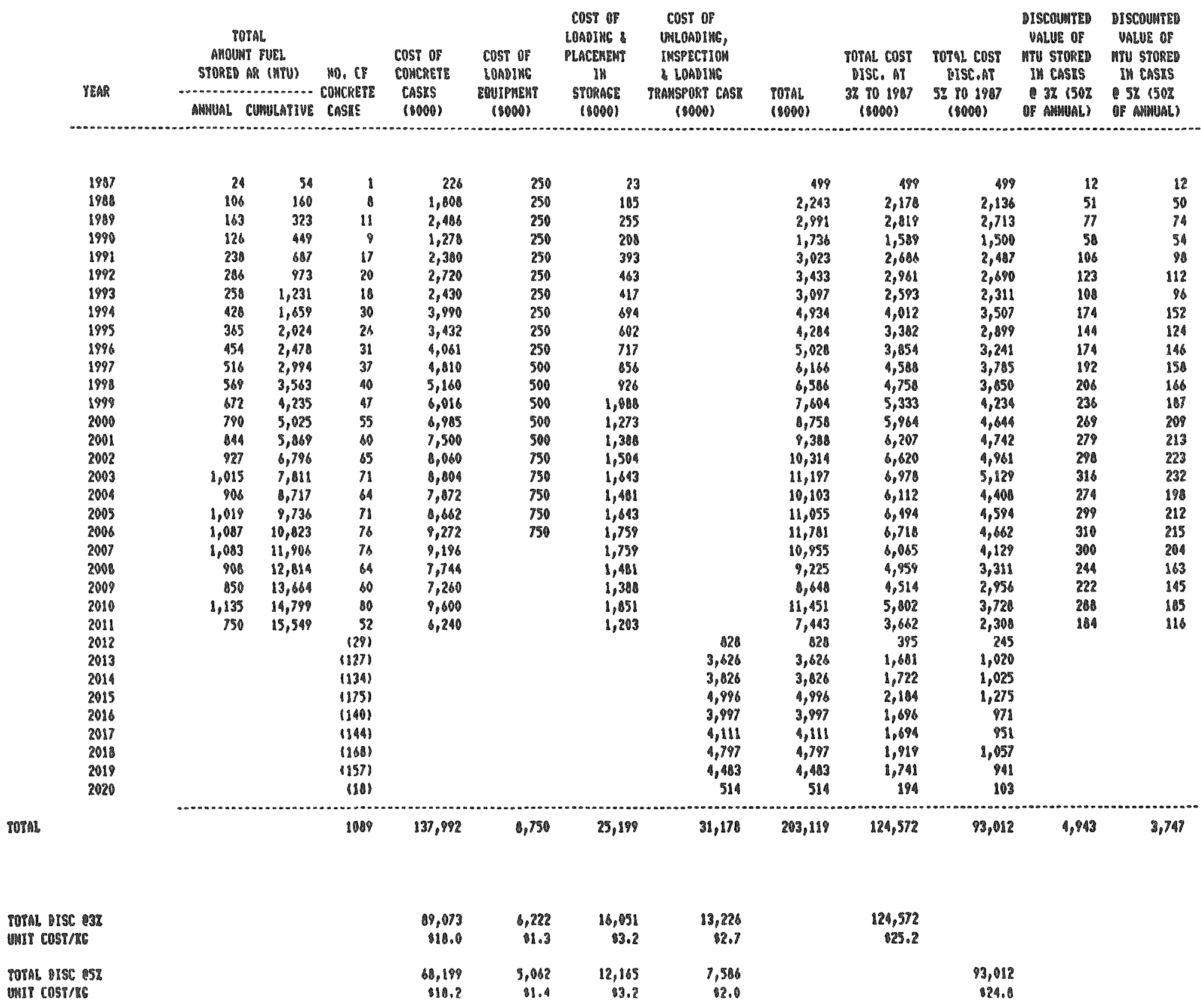


TABLE C-33

COMPARATIVE COSTS OF USE OF CASKS IN AT-REACTOR STORAGE OF INTACT FUEL ASSEMBLIES

(\$/KgU, 1987$)^{2}$

Unit Costs for Indicated Equipment or Activity

\begin{tabular}{|c|c|c|c|c|}
\hline Casks & $\begin{array}{l}\text { Loading } \\
\text { Equipment }\end{array}$ & $\begin{array}{l}\text { Loading } \\
\text { Placement } \\
\text { In Storage }\end{array}$ & $\begin{array}{l}\text { Removal } \\
\text { From Storage } \\
\text { \& Preparation } \\
\text { For shipment } \\
\end{array}$ & Total \\
\hline$\$ 87.9$ & $\$-$ & $\$ 0.7$ & $\$ 0.1$ & $\$ 88.7$ \\
\hline 78.8 & - & 0.7 & 0.1 & 79.6 \\
\hline 78.8 & - & 0.7 & 0.6 & 80.1 \\
\hline 78.8 & - & 0.7 & 2.1 & 81.6 \\
\hline 31.3 & 2.3 & 14.5 & 6.9 & 55.0 \\
\hline
\end{tabular}

\section{5-Year Repository Delay}

TSC Shipped Directly to DOE

0.7

0.1

85.3

Soc One-TIme Direct Shipment to $\mathrm{OOE}$

75.

0.7

0.1

76.2

SOC Shipped to DOE In Overpack

75.4

0.7

0.5

96.6

SOC Used for At-Reactor Storage

75.4

0.7

1.9

78.0

Concrete cask

29.8

2.0

14.5

6.3

52.6

10- Year Repository Delay

TSC Shipped Directiy to DOE

SOC One-Time Direct Shipment to DOE

soc shipped to DoE In Overpack

SOC Used for At-Reactor Storage only

concrete cask

Averaged at a 3\$/year discount rate

$\begin{array}{lcccc}82.8 & - & 0.7 & 0.1 & 83.4 \\ 73.8 & - & 0.7 & 0.1 & 74.6 \\ 73.8 & - & 0.7 & 0.5 & 75.0 \\ 73.8 & - & 0.7 & 1.8 & 76.3 \\ 29.0 & 2.0 & 14.5 & 5.8 & 51.3\end{array}$


TABLE C-34

COMPARATIVE COSTS OF USE OF CASKS IN AT-REACTOR STORAGE OF CONSOLIDATEO FUEL

$(\$ / \mathrm{kgu}, 1987)^{\mathrm{a}}$

Unit Costs for Indicated Equipment or Activity

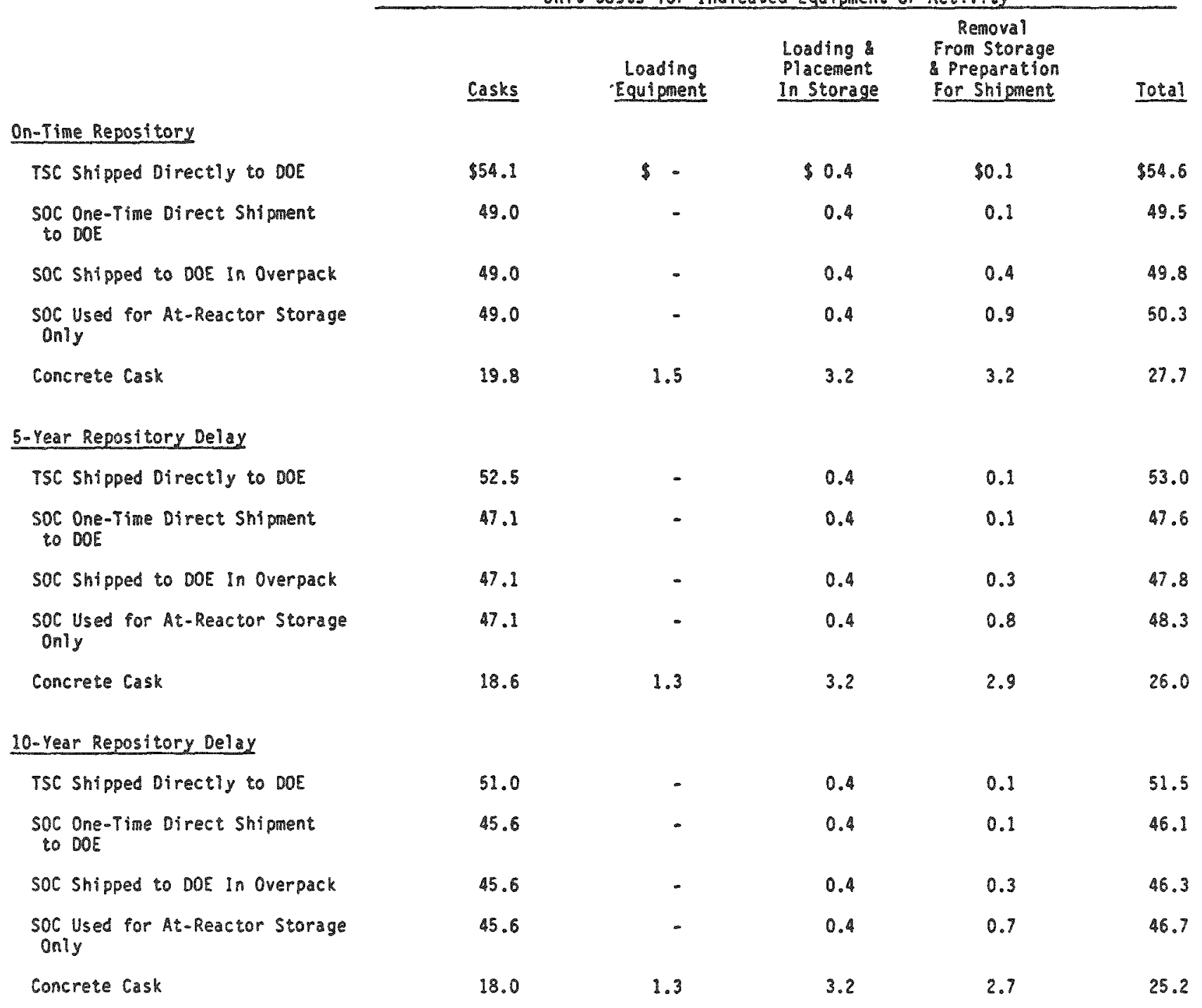

Averaged at a 3\%/year discount rate 
The following basic conclusions can be drawn from the results shown in Tables $\mathrm{C}-33$ and $\mathrm{C}-34$ with respect to the comparative costs of acquiring and handling TSCS, SOCS and concrete casks at the reactor site:

(1) TSCs cost about $\$ 7.2 / \mathrm{kgU}$ (or 9.1 percent) more than SOCs when used to store intact fuel assemblies, and about $\$ 4.6 / \mathrm{kgU}$ (or 9.5 percent) more than SOCs when used to store consolidated fuel -- if the SOC has to be unloaded at the end of the storage period and the contained fuel loaded into a DOE-supplied transport cask for shipment to a DOE facility.

(2) If the loaded SOCS can be Ticensed for a one-time shipment to DOE facilities, TSCs would cost about $\$ 9 / \mathrm{kgU}$ (or 11.7 percent) more than SOCs when used to store intact fuel assembilies, and about $\$ 5.3 / \mathrm{kgU}$ (or 11.1 percent) more than SOCs when used to store consolidated fuel.

(3) If the loaded SOCs must be overpacked for a one-time shipment to DOE facilities, TSCS would cost about $\$ 8.6 / \mathrm{kgU}$ (or 11.1 percent) more than SOCs when used to store intact fuel assemblies, and about $\$ 5.1 / \mathrm{kgU}$ (or 9.5 percent) more than SOCs when used to store consolidated fuel.

(4) TSCs cost about $\$ 33 / \mathrm{kgU}$ (or 62 percent) more than concrete casks when used to store intact fuel assemblies, and about $\$ 27 / \mathrm{kgU}$ (or 104 percent) more when used to store consolidated fuel.

However, it should be remembered that the costs associated with storage of spent fuel in concrete casks have not yet been demonstrated and thus the foregoing conclusions regarding the costs of such storage may be optimistic. In addition, other factors may make the concrete cask less desirable, such as:

(1) Higher land requirements

(2) Larger number of fuel handling operations

(3) Higher prospective worker exposure

(4) The prospective transportability of TSCS and SOCS -- which give a lesser impression of the permanence of storage

There are concrete storage cask designs that reportedly might be capable of being loaded in the reactor pool, thus eliminating the concerns outlined in (2) and (3), above, and which have the potential for further reducing the costs involved. The results of further demonstration work with concrete casks 
(both the NUHOMS horizontally loaded modules as well as the NUPAC vertically loaded cask) should help to clarify the relative desirability of concrete and metal casks.

It should also be recognized that there are also significant uncertainties about the cost of licensable TSCS as well as the feasibility of shipping SOCs either on a one-time basis or in overpacks. Thus the foregoing comparisons have a wide range of uncertainty associated with them. Nonetheless, the comparisons are indicative of the fact that the use of TSCS or SOCs for at-reactor storage of spent fuel may well result in significantly higher costs to utilities than alternative methods of dry storage -. which would have to be offset by savings in the DOE spent fuel management system resulting from the use of TSCS or SOCS, or other advantages of their use, in order for them to be viable.

3.0 REFERENCES FOR APPENDIX C

(1) C. M. Heeb, R. A. Libby, R.C. Walling and W. L. Purcell, Reactor-Specific Spent Fuel Discharge Projections; 1985 to 2020, PNL-5833, Pacific Northwest Laboratory, September 1986

(2) U. S. Department of Energy, Mission Plan for the Civilian Radioactive Waste Management Program, DOE/RW-0005, June 1985 
APPENDIX D

ESTIMATED COSTS FOR TRANSPORT OF SPENT FUEL FROM REACTOR SITES TO

DOE FACILITIES IN STANDARD TRANSPORT CASKS, TSCS, AND SOCS

(BOTH WITH AND WITHOUT OVERPACKS) 


\section{APPENDIX D}

ESTIMATED COSTS FOR TRANSPORT OF SPENT FUEL FROM REACTOR SITES TO DOE FACILITIES IN STANDARD TRANSPORT CASKS, TSCS, AND SOCS

(BOTH WITH AND WITHOUT OVERPACKS)

TABLE OF CONTENTS

1.0 ESTIMATED CAPITAL COSTS

D-1

1.1 Cost of Transport Casks

D-1

1.2 Cost of SOC Overpacks

$D-2$

2.0 ESTIMATED TRANSPORT COSTS

2.1 Freight Rates

2.2 Security Costs

D-2

D-2

2.3 Operating Costs

D-3

D-4

3.0 LIFE CYCLE TRANSPORT COSTS AND UNIT COSTS

D-5

4.0 REFERENCES FOR APPENDIX D 
The purpose of this Appendix is to develop estimates of the cost of transporting spent fuel from reactor sites to DOE facilities in (i) standard transport casks, (ii) TSCs, ( $i i i)$ SOCs used for a single shipment only, and (iv) SOCs used with a protective overpack, so that the extent of the benefits involved in shipping TSCs and SOCs over those for standard transport casks can be determined. The estimated capital and operating costs associated with the foregoing methods of shipment, and the estimated life cycle and unit costs of various shipment scenarios, are developed in the following sections.

\subsection{ESTIMATED CAPITAL COSTS}

1.1 COST OF TRANSPORT CASKS

For the purposes of this study, it was assumed that DOE-supplied transport casks would cost the same as a TSC. The cost of design and fabrication of TSCS has been previousiy discussed in Section 2.0 of Appendix $A$, and the cost of the first 10 such units (over which the design and development has been amortized) was estimated at \$1.319-miliion (Tabie A-4). In addition to the costs shown in that section, additional costs would be required for auxiliary and support equipment. These include the following:

\section{TABLE D-1}

ESTIMATED COST OF AUXILIARY EQUIPMENT REQUIRED

\section{TO SUPPORT OPERATION OF A TSC}

(\$000, 1987)

$\begin{array}{lr}\text { Description } & \frac{\text { Cost }}{\text { Rail Car, Personnel Barrier }} \\ \text { \& Tie Downs } & \$ 538 \\ \text { Impact Limiters } & 108 \\ \text { Lift Beam, Misc. Equipment } & 108 \\ \text { \& Special Tools } & \\ \text { Total } & \$ 754\end{array}$


The foregoing costs were based on estimates provided by designer/vendors and escalated to 1987 dollars. A cask that is used solely in transport service usually has the auxiliary equipment dedicated to its use. Thus, the cost of a transport cask is estimated to be \$2.073-million.

It should be pointed out here that a TSC or SOC used for storage of spent fuel would to have the auxilary equipment described in Table $D-1$ available to it whenever it is used in transport service. This means that while there may be scenarios where a TSC or SOC could be used to replace a transport cask, the auxiliary equipment described above would still be required.

\subsection{COST OF SOC OVERPACKS}

For the purposes of this study, it was assumed that an overpack for use with a SOC would cost about \$0.38-million, and that the cost of design and development thereof would amount to about \$2-million. It was further assumed that the latter cost would be spread over 10 units, thus giving a total cost of the overpack of \$0.58-million. Here again, as in the case of the TSC, the auxiliary equipment described in Table D-1 would be required for the shipment of overpacks.

The weight of the overpack loaded with a SOC containing spent fuel was estimated to be 324,500 1bs; the empty overpack was estimated to weigh 92,5001 bs.

\subsection{ESTIMATED TRANSPORT COSTS}

The costs for transport of spent fuel from reactors to DOE facilities was estimated for three different distances 1300 miles, 900 miles and 2300 miles) in order to obtain a range of expected costs. The following sections describe the development of the component costs.

\subsection{FREIGHT RATES}

PNL has recently developed truck and rail charges for spent fuel for a number of origin and destination combinations (Reference 1), based on the assumption that the casks would be transported at general commodity rates (Class 40$)$. The rail freight rates contained in the PNL report were used to develop a relationship of distance to freight rate, as follows: 


$$
\begin{aligned}
& R_{1}=0.1678 D^{0.5815} \\
& R_{e}=0.1600 D^{0.5790}
\end{aligned}
$$

where $\quad R_{1}=$ loaded cask freight rate expressed in $\$ / C W T$

$R_{e}=$ empty cask freight rate expressed in $\$ / C W T$

$D=$ one-way distance

While the PNL freight rates were in 1986 dollars, they were not escalated inasmuch as the price index for rail freight has declined slightly during 1986; it was concluded that the 1986 values should be applicable for 1987 as well.

The freight rates determined using the foregoing relationships were as follows:

\begin{tabular}{crr}
$\begin{array}{c}\text { Distance } \\
\text { (Miles) }\end{array}$ & \multicolumn{2}{c}{ Freight Rate $(\$ / C W T)$} \\
\cline { 2 - 3 } 300 & Loaded & Empty \\
900 & $\$ 4.63$ & $\$ 4.35$ \\
2300 & 8.76 & 8.22 \\
& 15.12 & 14.14
\end{tabular}

In computing the total freight charges for an individual shipment, it was assumed that the railroads would apply a $250,000 \mathrm{lb}$ minimum weight to a loaded cask shipment and a 225,000 lb. minimum weight to an empty cask shipment. However, for cases where the rail car, impact limiters, etc. were shipped to the reactor to pick up a loaded TSC or SOC, a 40,000 $1 \mathrm{~b}$ minimum weight and the empty cask freight rate was assumed to be acceptable to the railroads. (The foregoing conclusion was reached after discussing the matter with the Rockwell-Hanford traffic manager.) In cases where the empty overpack is returned to the reactor, a $132,500 \mathrm{lb}$. weight and the empty cask freight rate were assumed to be applicable.

\section{$2.2 \quad$ SECURITY COSTS}

Under existing NRC regulations, shipments of spent fuel must be escorted by armed guards. The cost for these escorts was developed using the following assumptions: 
(1) Two men (a guard sergeant and an officer) would be located in the caboose during the entire loaded portion of the shipment. The sergeant would carry a rate of $\$ 12.56 / \mathrm{hr}$ and the officer a rate of $\$ 11.42 / \mathrm{hr}$. Each would receive 16 hours of pay for each 24 hours involved in the shipment. The guards' pay would carry a 35 percent burden; a 13 percent general administrative cost would be applied to the salary rate plus burden; and a 5 percent profit would be applied to the sum of the foregoing. This produces a total of $\$ 615 /$ day for the escorts. This cost was applied to the total elapsed time of the shipment plus a 16 hour layover, air travel at $500 \mathrm{mph}$, and 4 hours for ground travel time.

(2) Each of the guards would each receive a $\$ 25 /$ day subsistence allowance.

(3) In addition to (2), above, the guards would receive $\$ 70$ for hotel and taxi expenses on the return trip.

(4) A rail fare of $\$ .085 / \mathrm{mile}$ per guard was assumed to be applicable to the shipment. Air fare was estimated at $\$ 0.30 /$ mile per guard for the return trip.

(5) A security equipment charge of $\$ 12 /$ day (total) was assumed to be applicable.

These costs were developed by escalating similar costs used to develop estimated transportation costs in the 1983 FIS Fee Report (Reference 2) by the applicable indexes. Using the above costs, the security costs associated with a single 300-mile shipment would amount to $\$ 5,273$, for a 900-mile shipment would amount to $\$ 8,372$, and for a 2300-mile shipment would amount to $\$ 11,160$.

\subsection{OPERATING COSTS}

Most of the operating costs associated with the use of a transport cask, a TSC or a SOC have been included in the cost of operations at the reactor site (Appendix $B$ ) and at the DoE receiving facilities. Thus the only cost not otherwise covered is that for maintenance of the transport cask and the associated auxiliary support equipment.

The annual cost of maintenance of the transport cask and associated auxiliary equipment was estimated to be $\$ 60$-thousand (1987 dollars). In cases where a TSC or SOC is used for a one-way shipment, no maintenance cost was ascribed to the cask, but the annual cost of maintaining the auxiliary equipment was estimated to be $\$ 10$-thousand (1987 dollars). In cases where an 
overpack is used in a SOC shipment, the annual maintenance cost was assumed to be $\$ 35$-thousand (1987 dollars).

$3.0 \quad$ LIFE CYCLE TRANSPORT COSTS AND UNIT COSTS

The total life cycle costs associated with the use of a single transport cask, or the equivalent number of TSCS or SOCS, for shipment of spent fuel from the reactors to DOE facilities were developed for the following cases:

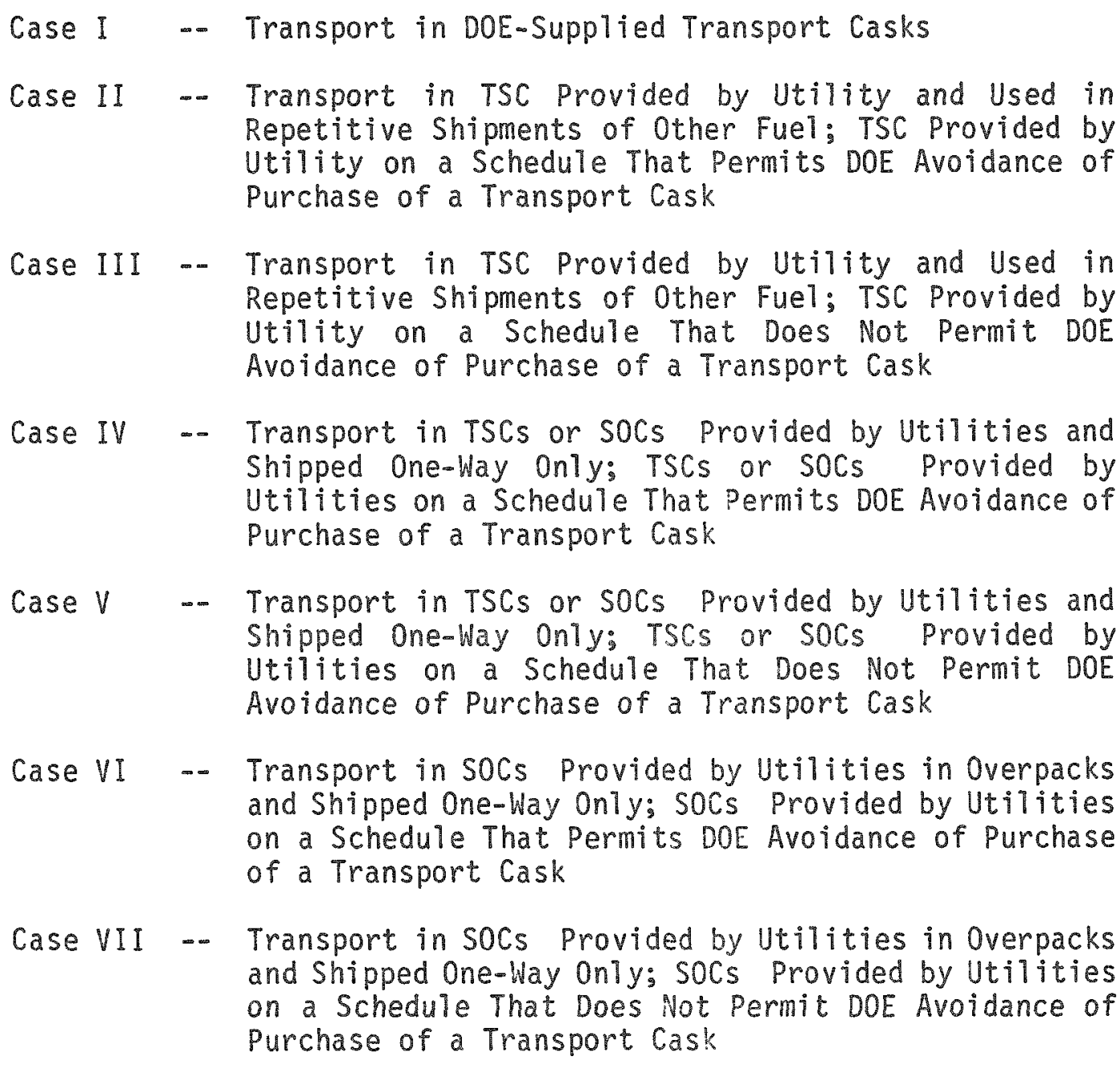

For each of the foregoing cases, life cycle costs were develop for transport over distances of 300 miles, 900 miles, and 2300 miles (one-way). These are designated as subcases $A, B$ and $C$, respectively for each of the cases described above. 
The life cycle costs and resulting unit costs for each of the foregoing cases are shown in Table D-2 through D-22. A summary of the unit costs is set forth in Table D-23. The figures set forth in the Tables were developed as follows:

(1) The total costs were assumed to be incurred over the period 1996 to 2023 inclusive. In 1996 and 1997 the costs of acquiring needed equipment would be incurred; during the period 1998 through 2022 the cost of operations would be incurred; and in 2023 the cost of decommissioning the transport cask and/or overpack would be incurred.

(2) The annual quantities shipped each year for 25 years were assumed to be as follows:

$$
\begin{aligned}
& 300 \text { miles - } 176 \text { MTU/year } \\
& 900 \text { miles - } 118 \text { MTU/year } \\
& 2300 \text { miles }-98 \text { MTU/year }
\end{aligned}
$$

The foregoing were based on an average cask capacity of 9.26 MTU, an average cask availability of 300 days/yr., a total turnaround time of 72 hours for each round trip cask shipment, and the following rail speeds (Reference 1 ):

\begin{tabular}{cc} 
Distance & Miles/Day \\
\cline { 2 - 2 } $0-300$ & 47 \\
$301-1100$ & 88 \\
$1101-1900$ & 143 \\
$1901-2400$ & 182
\end{tabular}

(3) The capital costs used were as follows:

(a) For cases involving the use of a DOE-supplied transport cask (Case I), the capital costs used was \$2.073-million (see Section 1.1).

(b) For cases involving use of a TSC or SOC where commitments are made by utilities to deliver casks in sufficient quantities and on a schedule that permits DOE to avoid purchasing a transport cask (Cases II and IV), the capital cost used was \$1.149-million (\$0.754-million for auxiliary equipment plus $\$ 0.395$-million for the pro rata share of the transport cask development costs).

(c) For cases involving the use of TSCS or SOCS were DOE cannot avoid the purchase of a transport cask even though the use of it may be displaced by TSCS or SOCs (Cases III and $V$ ), the capital cost used was \$2.073-million (see Section 1.1). 
(d) For cases involving the use of a SOC in an overpack where commitments are made by utilities to deliver casks in sufficient quantities and on a schedule that permits DOE to avoid purchasing a transport cask (Case VI), the capital cost used was \$1.729-million $(\$ 0.754-m i l l i o n$ for auxiliary equipment, plus $\$ 0.395-m i l l i o n$ for the pro rata share of cask development costs for the displaced transport cask, plus \$0.58-million for the overpack).

(e) For cases involving the use of SOCs in overpacks where DOE cannot avoid the purchase of a transport cask even though use if it may be displaced by TSCs or SOCs (Case VII), the capital cost used was \$2.653-million.

(4) The operating costs used were as follows:

(a) For cases involving the use of a DOE-supplied transport cask, or TSCs used in repetitive shipments, (Cases I, II, and III) the annual operating cost used was \$60-thousand.

(b) For cases involving the use of TSCS or SOCs (without an overpack) in one-way shipments (Cases IV and $V$ ), the annual operating cost used was $\$ 10$-thousand.

(c) For cases involving the use of SOCs in overpacks (Cases VI and VII), the annual operating cost used was \$35thousand.

(5) The freight charges used were those set forth in Section 2.1 applied against the following weights:

(a) For cases involving the use a DOE-supplied transport cask, or TSCs used in repetitive shipments, (Cases I, II and III) $-250,000$ lbs 1oaded and 225,000 1bs empty.

(b) For cases involving the use of TSCS or SOCs (without an overpack) in one-way shipments (Cases IV and V) $--250,000$ lbs loaded and 40,000 lbs empty.

(c) For cases involving the use of socs in overpacks (Cases VI and VII) $-324,500$ lbs loaded and 132,500 lbs empty.

(6) The security (escort) charges used were those set forth in Section 2.2 .

(7) The cost of decommissioning a DOE-supplied transport cask or SOC overpack after use was assumed to be $7 \%$ of the cost thereof, excluding development, design, certification and auxiliary equipment. No decommissioning cost was ascribed to auxiliary equipment or to TSCS (the latter has been estimated in Appendix $H$ ). 
(8) The total costs were discounted by $3 \% /$ year to 1987 to get the total discounted costs.

(9) The unit costs were determined by dividing the total discounted costs by the total discounted amount (MTU) of spent fuel shipped, as follows:

Discounted Costs $=$ Discounted (Unit Costs $\times$ MTU Shipped)

Discounted Costs $=$ Unit Costs $\times$ Discounted MTU Shipped

Unit Cost $=$ Discounted Costs Discounted MTU Shipped 
IAL -2

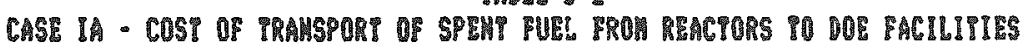
300 MILES AUAY - IN DOE-SUPPUIPD TRAMSPOKI CASY

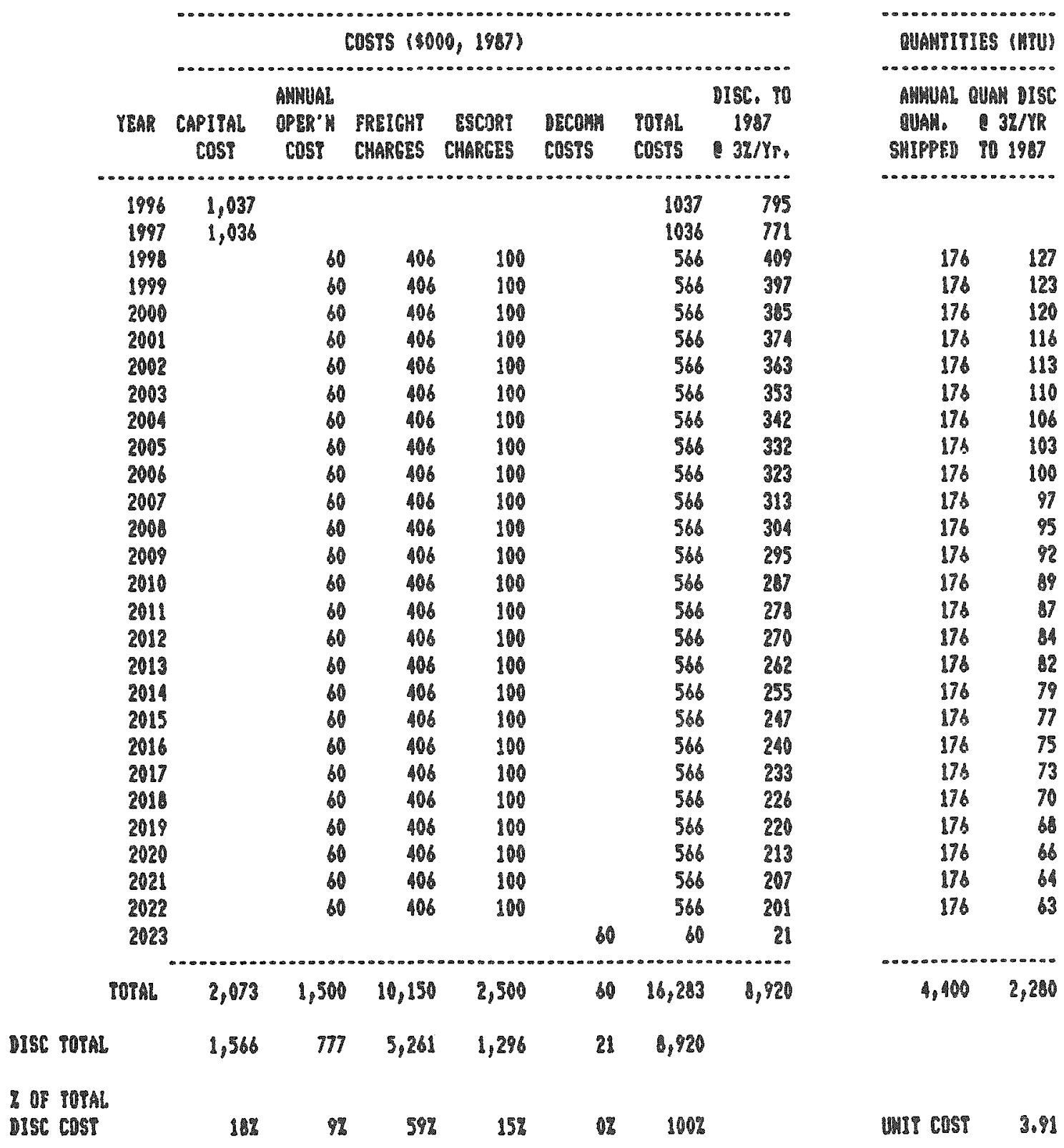


IABLE D-3

CASE IN - COST OF TRAMSPORY OF SPEMT FUEL FKOH REACYORS IO DOP BACULIPIES 900 MILES AMAY -. IN DOE-SUPPLIED TRAHSPOAT CASK

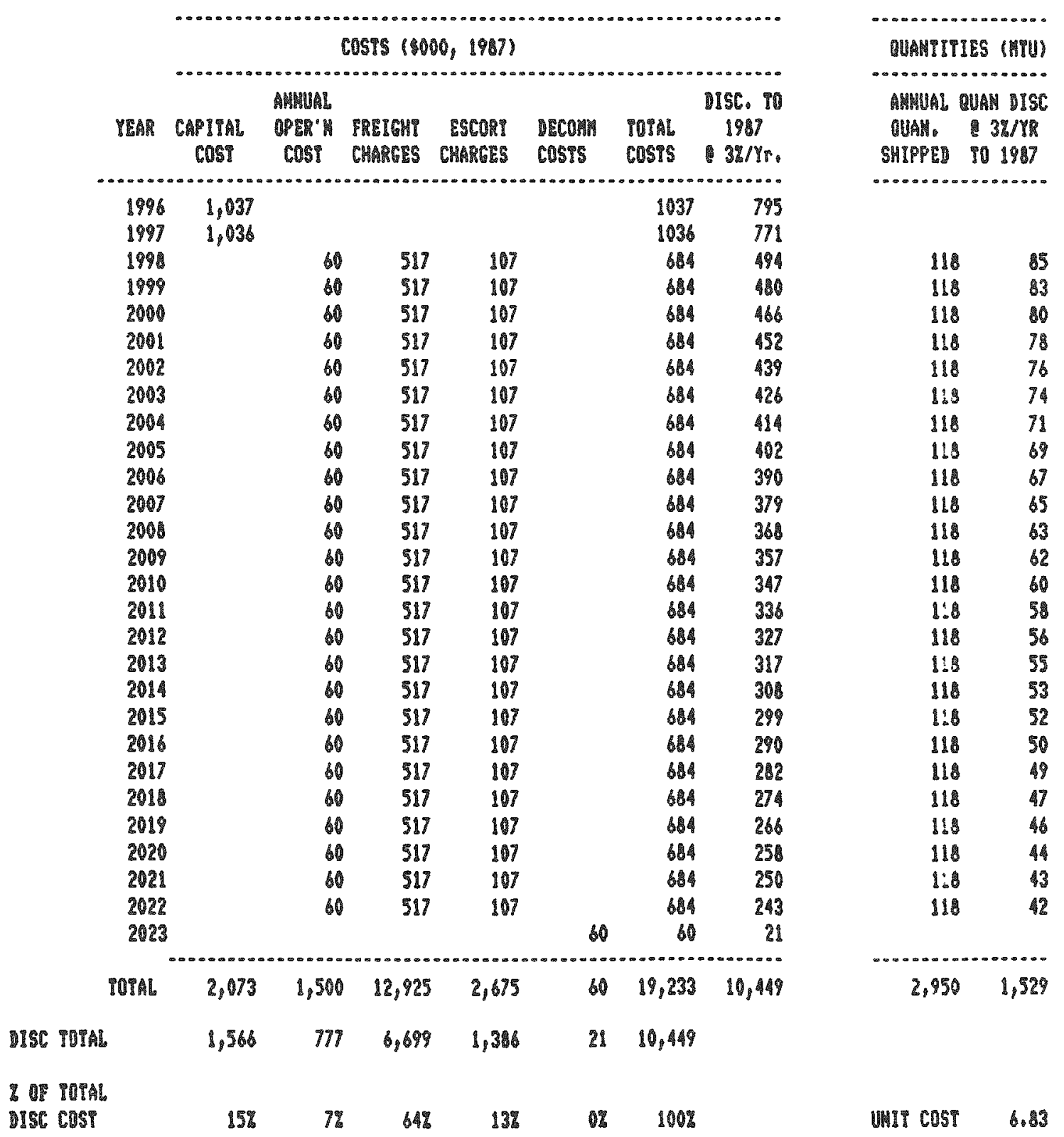


TASLE D-

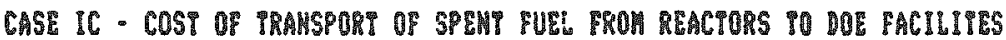
2300 MILES AHAY -. IN DOC-SUPPLIED TRAMSPORT CAS

\begin{tabular}{|c|c|c|c|c|c|c|c|}
\hline Yen & $\begin{array}{c}\text { CAPIIALL } \\
\text { Cos: }\end{array}$ & $\begin{array}{l}\text { AWWUAL } \\
\text { OPERR' } \\
\text { COSP }\end{array}$ & $\begin{array}{l}\text { FREIGHT } \\
\text { CHARGES }\end{array}$ & $\begin{array}{r}\text { ESCORY } \\
\text { CHARGES }\end{array}$ & $\begin{array}{l}\text { Dedom } \\
\text { COSTS }\end{array}$ & $\begin{array}{l}\text { TOIAL } \\
\text { Costs }\end{array}$ & $\begin{array}{l}\text { DISC. To } \\
1987 \\
3 \% / Y r\end{array}$ \\
\hline 1996 & 1,037 & & & & & 1037 & 795 \\
\hline 1997 & 1,036 & & & & & 1036 & 772 \\
\hline 1998 & & 60 & 739 & 118 & & 917 & 662 \\
\hline 1999 & & 60 & 739 & 118 & & 918 & 643 \\
\hline 2000 & & 60 & 799 & 118 & & 917 & 824 \\
\hline 2001 & & 60 & 739 & 118 & & 917 & bos \\
\hline 2002 & & 60 & 739 & 110 & & 917 & 589 \\
\hline 2003 & & 60 & 739 & 118 & & 917 & 371 \\
\hline 2004 & & 60 & 739 & 110 & & 917 & 555 \\
\hline 2005 & & 80 & 739 & 118 & & 917 & 539 \\
\hline 2006 & & 80 & 739 & 118 & & 917 & 523 \\
\hline 2007 & & 80 & 739 & 118 & & 917 & 308 \\
\hline 2008 & & 60 & 739 & 118 & & 917 & 493 \\
\hline 2009 & & 60 & 739 & 118 & & 917 & 179 \\
\hline 2010 & & 60 & 739 & 118 & & 917 & 465 \\
\hline 2011 & & 60 & 739 & 118 & & 917 & 451 \\
\hline 2012 & & 60 & 739 & 118 & & 917 & 431 \\
\hline 2013 & & 60 & 739 & 118 & & 917 & 425 \\
\hline 2014 & & 60 & 739 & 110 & & 917 & 413 \\
\hline 2015 & & 80 & 739 & 118 & & 917 & 301 \\
\hline 2016 & & 60 & 739 & 118 & & 917 & 369 \\
\hline 2017 & & 80 & 739 & 118 & & 917 & 378 \\
\hline 2018 & & 80 & 739 & 118 & & 917 & 367 \\
\hline 2019 & & 80 & 739 & 118 & & 917 & 356 \\
\hline 2020 & & 80 & 739 & 118 & & 917 & 346 \\
\hline 2021 & & 80 & 739 & 118 & & 917 & 336 \\
\hline 2022 & & 80 & 739 & 118 & & 917 & 326 \\
\hline 2023 & & & & & 80 & 60 & 21 \\
\hline TOTAL & 2,073 & 1,500 & $180_{\beta} 475$ & 2,850 & 80 & 25,058 & 13,460 \\
\hline & 1,566 & $y$ & 9,575 & 1,529 & 21 & 13,480 & \\
\hline
\end{tabular}

QUAMTITIES IFTU

AHWUAL QUAN DISC

DUAH. $3 \% / K R$

SHIPPEY TO 1987

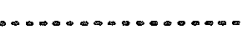

98

9669

9867

$98 \quad 65$

963

$98-31$

98

915

98

965

95

91

9850

98

94217

96. 45

98

9443

9242

818

79

$98 \quad 38$

9637

9836

93

$2,450 \quad 1,270$

DISC FOTAL

$\begin{array}{llllll}1,566 & 777 & 9,575 & 1,589 & 21 & 13,486\end{array}$

2 OP POTAL

DSC COST

$122 \quad 71 \% \quad 11 \% \quad 0 \% \quad 100 \%$

UMIT COST 10.61 
TARLE D-

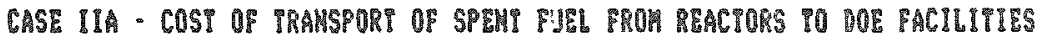

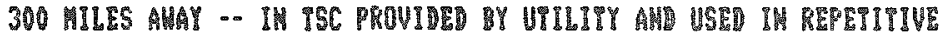

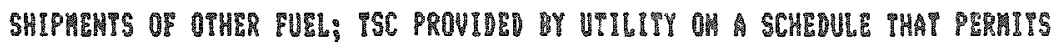
DOE AVODAMCE OF PURCHASE OP TRAHSPORT CASY

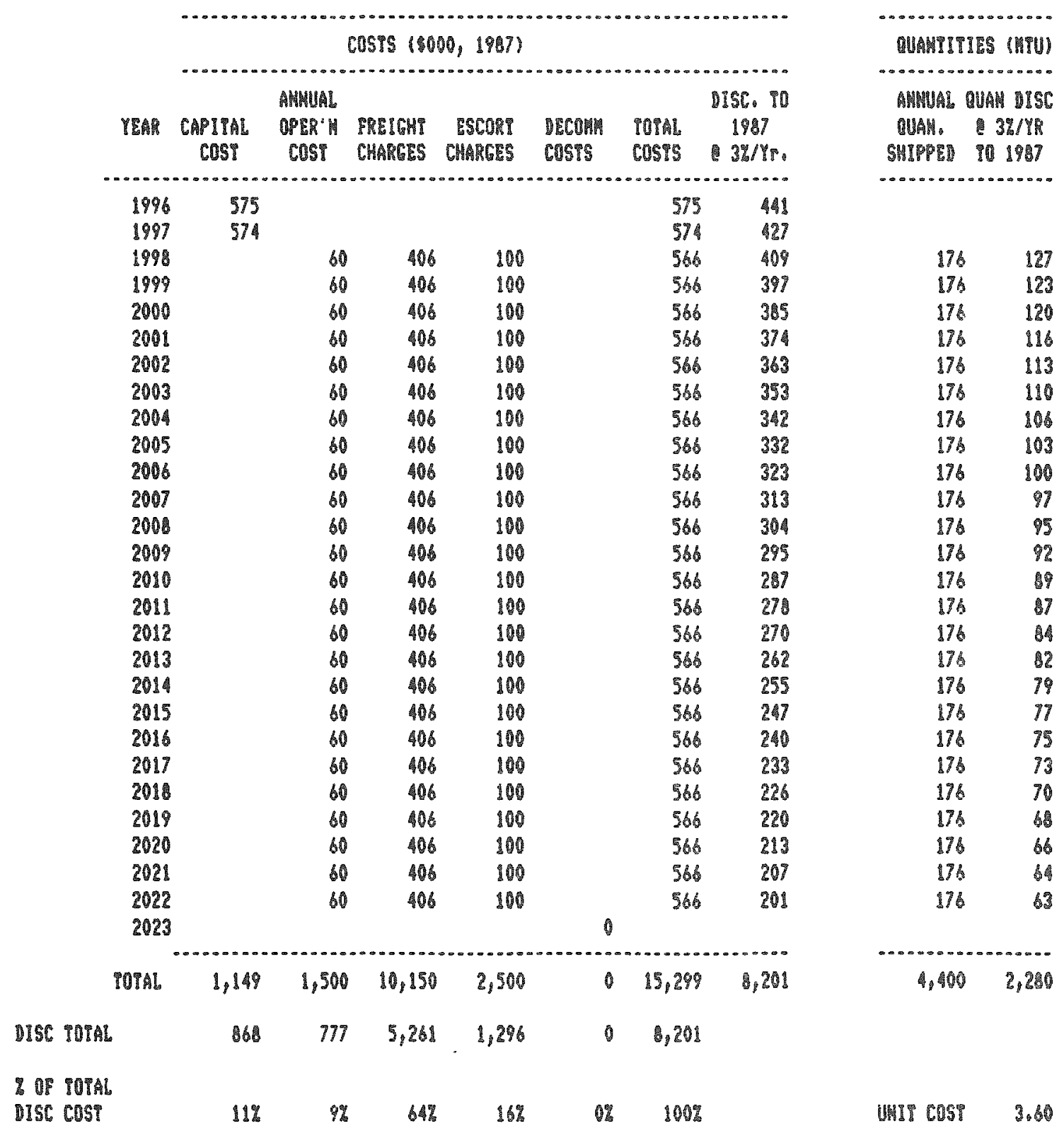


PABSLID D-6

CASE IIS - COST OP TRAMSPORT OF SPRM FUEL PROM REACTORS PO DOP FACILITIES

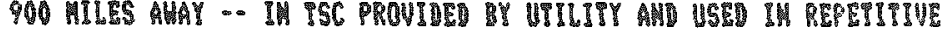
SHIPHEMTS OF OTHER YUEL ISC PROUDDE BY UTLITY OH A SCHEDULE IHAT PERITS DOE AUOIDAMCE OP PURCHASE OF A TRAMSPORT CASH

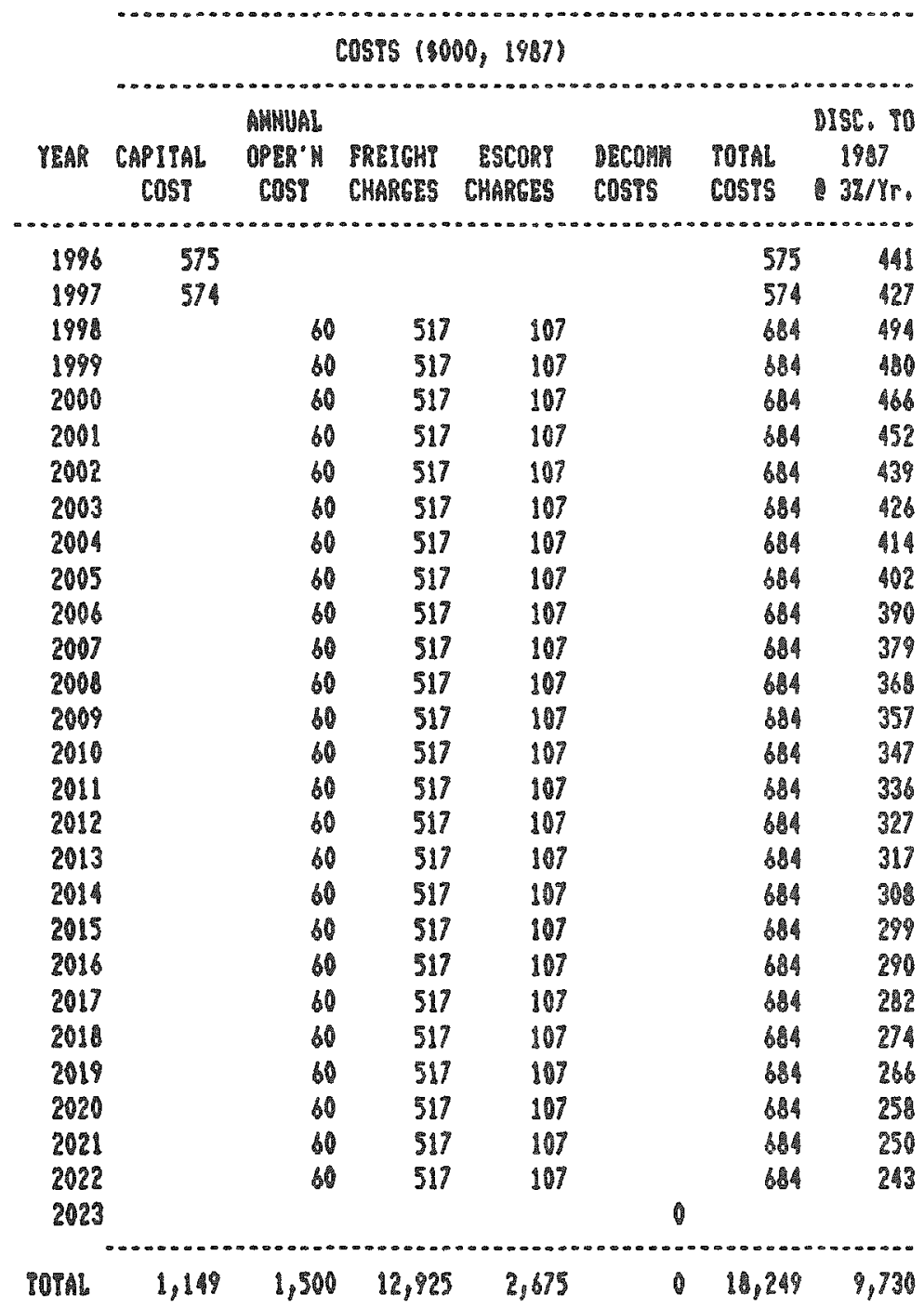

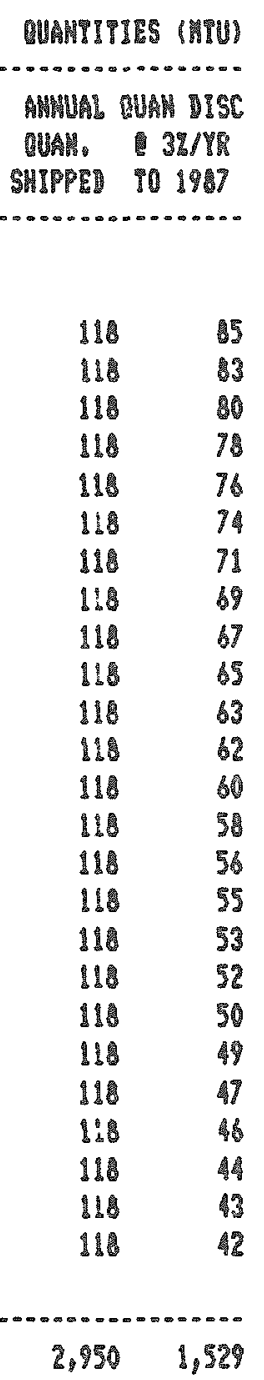

DISC TOTAL

$960 \quad 777 \quad 6,699 \quad 1,396 \quad 9,730$

\% OP POÁl ISC COST 
TABLE D-7

CASE IIC - COST OP IRAMSPORT OO SPEMT BUEL PROA REACTORS IO DOE PACTLITIES 2300 HILES AUAY - IN ISC PRDUIDED BY UTILITY AHD USED IM REPETIITUE

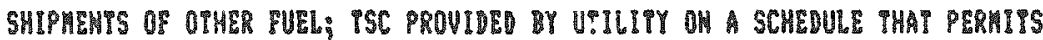
DOR AVOIDAMCE Of PURCHASE OR A TRAMSPORT CASK

\begin{tabular}{|c|c|c|c|c|c|c|c|}
\hline YEAR & $\begin{array}{c}\text { CAPIPAL } \\
\text { COSI }\end{array}$ & $\begin{array}{l}\text { AHMUAL } \\
\text { OPER'H } \\
\text { COSP }\end{array}$ & $\begin{array}{l}\text { PREIGH? } \\
\text { CHARGESS }\end{array}$ & $\begin{array}{l}\text { ESCORI } \\
\text { CHARGES }\end{array}$ & $\begin{array}{l}\text { Deconh } \\
\text { costs }\end{array}$ & $\begin{array}{l}\text { TOPAL } \\
\text { COSTS }\end{array}$ & $\begin{array}{c}\text { DISC. TO } \\
198 ? \\
3 \% / Y \text { r. }\end{array}$ \\
\hline 1996 & 575 & & & & & 575 & 441 \\
\hline 1897 & 574 & & & & & 574 & 427 \\
\hline 1998 & & 80 & 739 & 111 & & 917 & 662 \\
\hline 1899 & & so & 739 & 118 & & 917 & 643 \\
\hline 2000 & & 80 & 739 & 110 & & 917 & 624 \\
\hline 2001 & & 60 & 739 & 118 & & 817 & 608 \\
\hline 2002 & & 60 & 739 & 118 & & 917 & 589 \\
\hline 2003 & & 60 & 739 & 118 & & 817 & 571 \\
\hline 2004 & & 60 & 739 & 118 & & 917 & 555 \\
\hline 2005 & & 60 & 739 & 118 & & 817 & 539 \\
\hline 2006 & & 60 & 739 & 118 & & 917 & 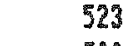 \\
\hline 2007 & & 60 & 739 & 118 & & 817 & 503 \\
\hline 2000 & & 60 & 739 & 118 & & 917 & 493 \\
\hline 2008 & & 60 & 739 & 118 & & 917 & 47 \\
\hline 2010 & & 60 & 739 & 118 & & 917 & . \\
\hline 2011 & & 60 & 739 & 118 & & 917 & 6 \\
\hline 2012 & & 60 & 739 & 118 & & 917 & 438 \\
\hline 2013 & & 60 & 739 & 118 & & 917 & 425 \\
\hline 2014 & & 60 & 739 & 118 & & 917 & . \\
\hline 2015 & & 60 & 739 & 118 & & 917 & 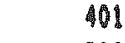 \\
\hline 2016 & & 60 & 739 & 118 & & 917 & 99 \\
\hline 2017 & & 60 & 739 & 118 & & 917 & 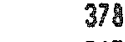 \\
\hline 2018 & & 60 & 739 & 118 & & 917 & 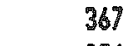 \\
\hline 2019 & & 80 & 739 & 118 & & 917 & 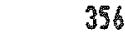 \\
\hline 2020 & & 60 & 739 & 118 & & 917 & 34 \\
\hline 2021 & & 80 & 739 & 118 & & 917 & 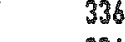 \\
\hline 2022 & & 60 & 739 & 118 & & 917 & 32 \\
\hline 2023 & & & & & 0 & & \\
\hline TOTAL & 1148 & 1,500 & 18,475 & 2,950 & 0 & 24,074 & 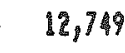 \\
\hline
\end{tabular}

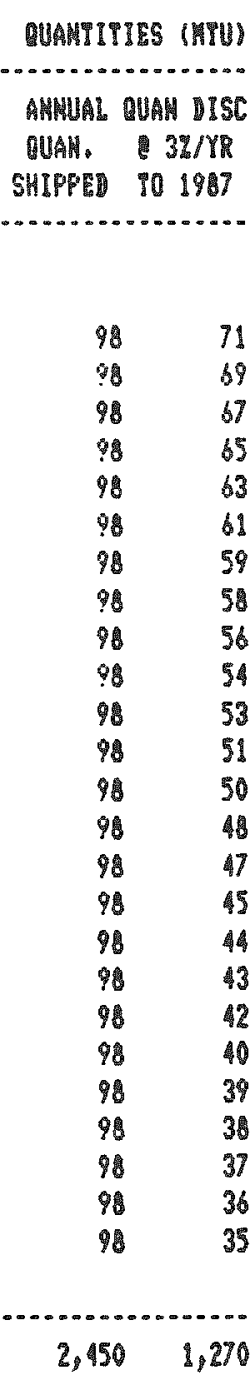

DISC TOTAL

868

$777 \quad 9,575 \quad 1,529$

- 12,798

IO TOTAL DISC COST

$7 \% \quad 6 \% \quad 75 \% \quad 12 \% \quad 0 \% \quad 100 \%$

UMIT COSI 10.04 
IABLE D-S

CASE IIIA - COST OP TRALPORT OF SPEMT FULL PROH RRAGTORS TO DOE PACILITIES 300 MILES AWAY - IN TSC PROUIDED IY UILLIYY AHI USED IN REPETIIUE SHIPMEMTS OF OTHER PUEL ISC PROUIDE PY UIILITY ON SCHEULE THAT DOES NOP PRRMIY DOE AUODOACE OF PURCHASE OF 1 PRAMSPOR CASY

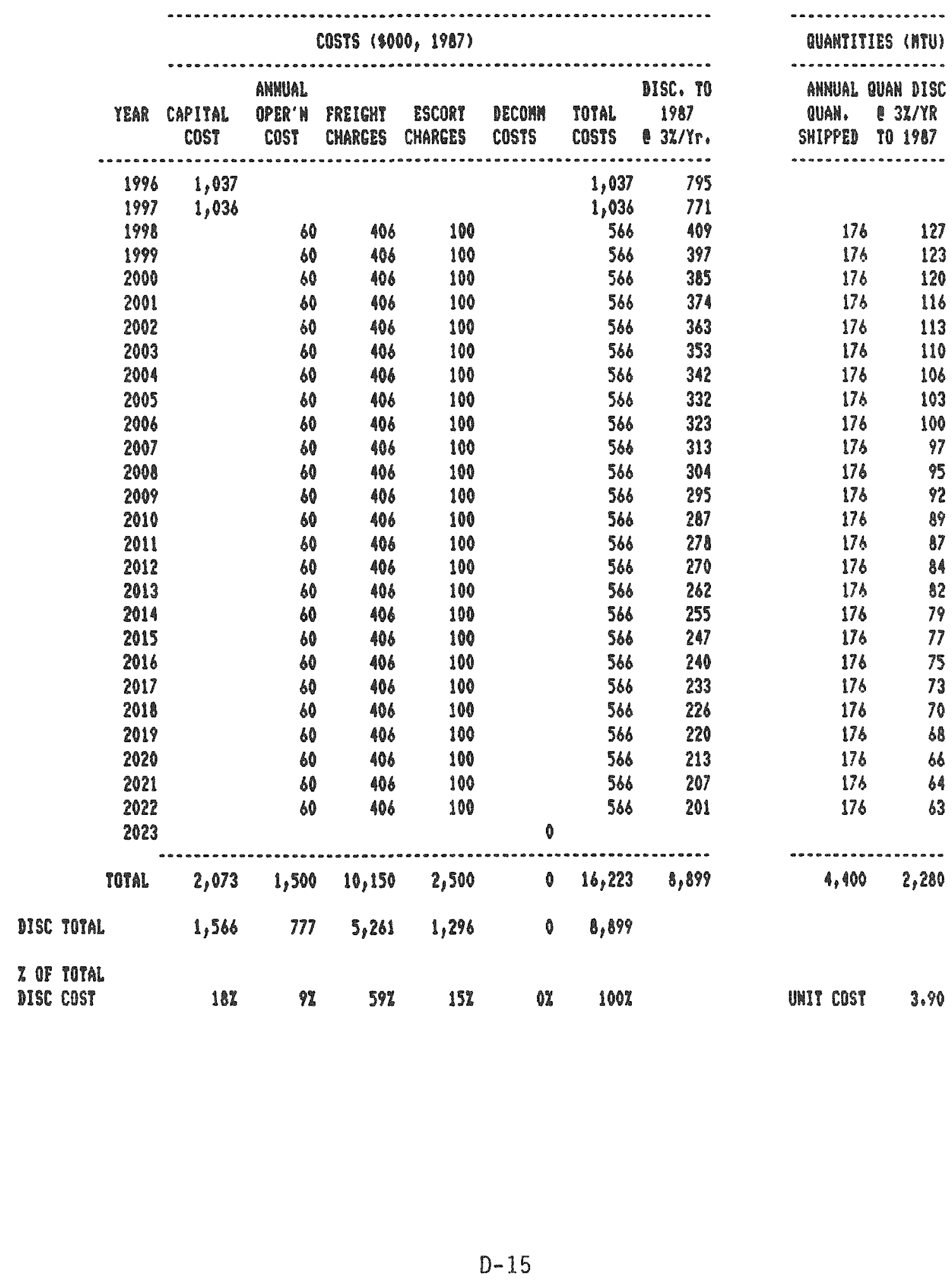


TABLL DOS

CASE III - COST OF PRAHSPORT OR SPEHT FUEL FROH REACTORS TO DOE FACTLITIES 900 MILES AUAY -. IN ISC PROVIDED BY UIILITY AHD USED IN REPEIIITUR SHPPMENTS OF OTHER PUEL; ISC PROUIDED BY U.ILITY OH A SCHEDULE PHAP DOES NOT PRRMII DOE AVOTDAMCE OF PURCHASE OF A TRAMSPORT CASY

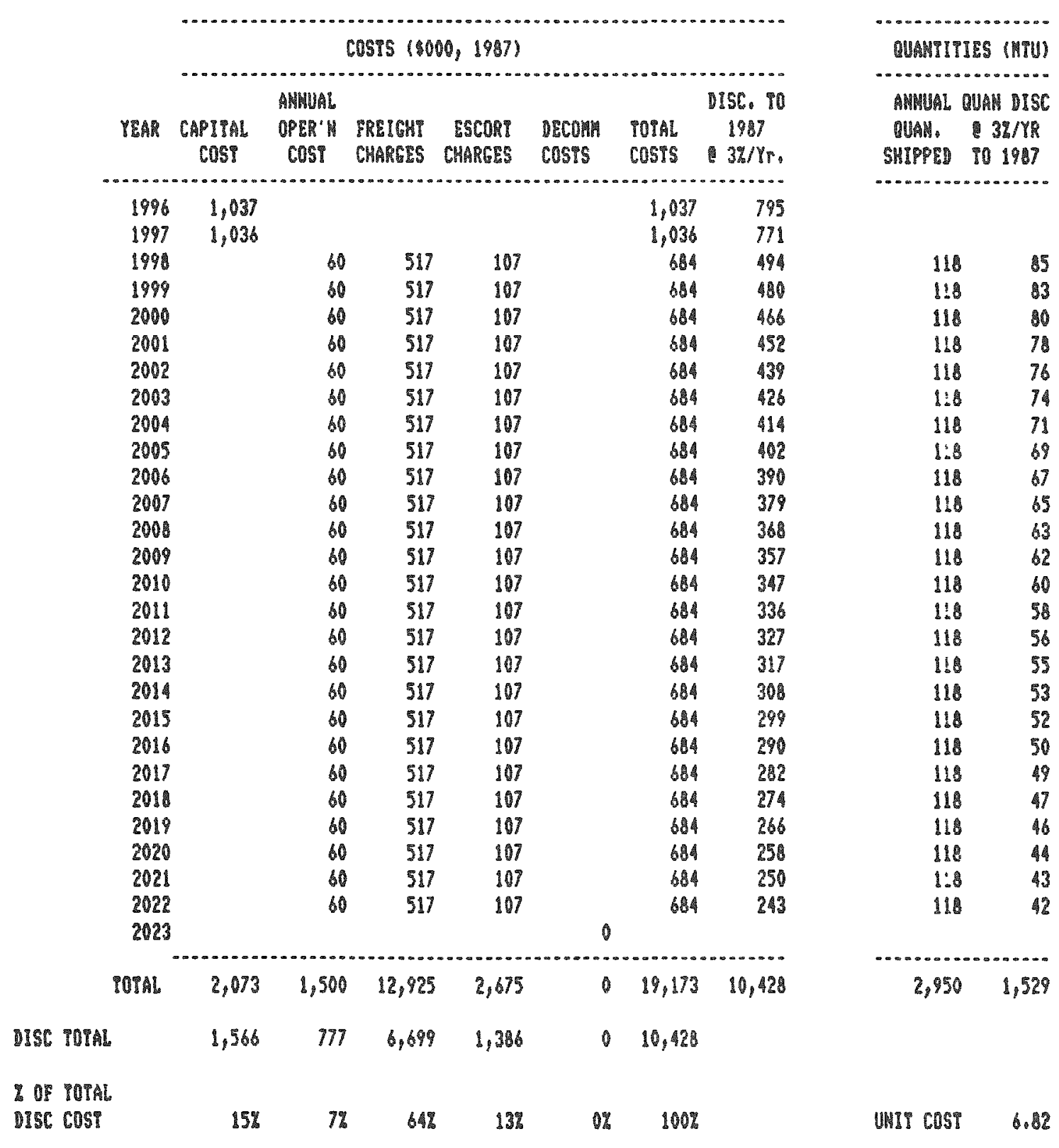


TAMLE D-10

CASE IIIC - COST OS TRAMSPORT OF SPTHT FULL PRON REACTORS TO DOE FACLIIIIES

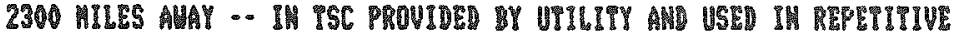

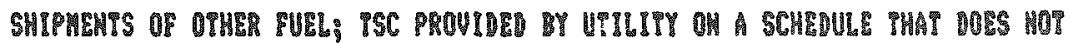

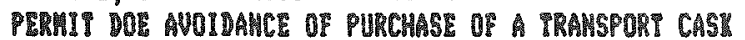

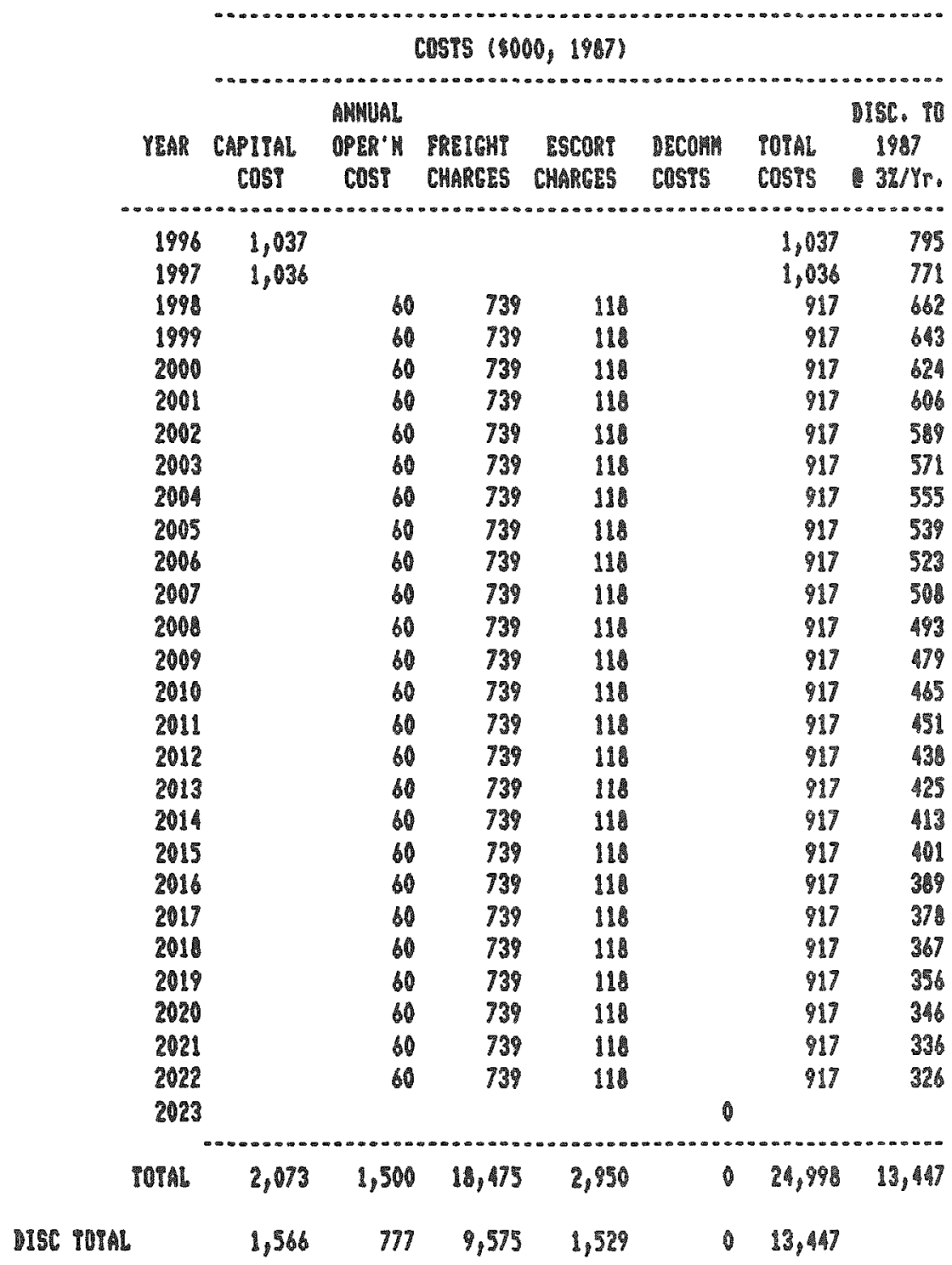

MดTITIS (MTU)

ARMUAL OUN DISC

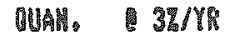

SHIFFE 1987

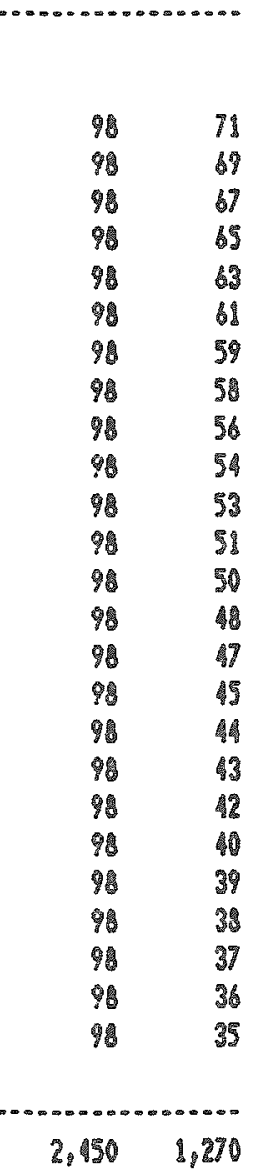

2) Of TOTAL

DISC COSP

$122 \quad 6 \% \quad 71 \% \quad 11 \% \quad \% \quad 100 \%$

Uม1 cos! 10.59 
IABLI D-11

CASE IUA - COST OF TRAMSPORT OF SPEMI FUEP PROH REACTORS TO DOE FACILITIES

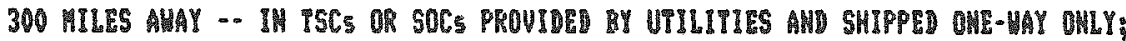

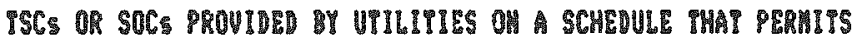

DOC AUOIDANC OF PURCHASE OF TRABSPOR CASE

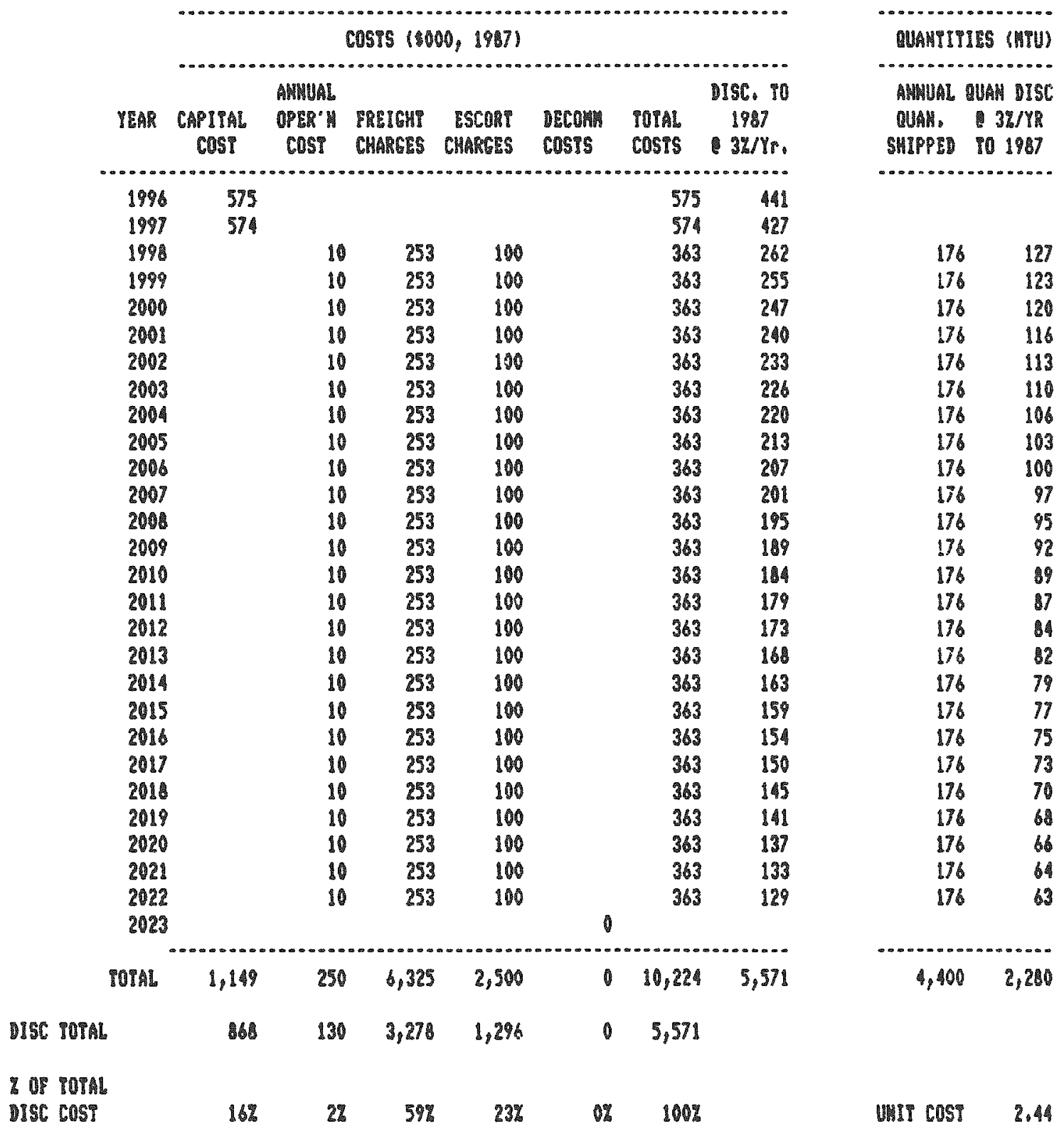


TARLE D-12

CASE UU - COST OF TRAHSPOR OF SPEHT PUEL PRON REACTORS TO DOE FACILITIES

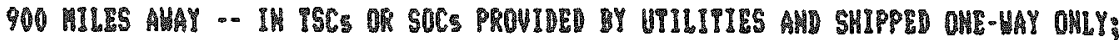

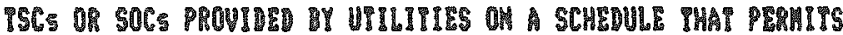

DOS AUODAACE OF PURCHASE OF A TRAMSPORT CASY

\begin{tabular}{|c|c|c|c|c|c|c|c|}
\hline \multicolumn{8}{|c|}{ CosTs 19000,1987$)$} \\
\hline YEM & $\begin{array}{c}\text { CMPITAL } \\
\text { COST }\end{array}$ & 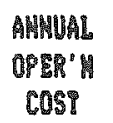 & $\begin{array}{l}\text { POEIGHI } \\
\text { CHARGES }\end{array}$ & $\begin{array}{r}\text { ESCORT } \\
\text { CHAREES }\end{array}$ & $\begin{array}{l}\text { DECOMY } \\
\text { COSTS }\end{array}$ & $\begin{array}{l}\text { POTAL } \\
\text { COSIS }\end{array}$ & $\begin{array}{c}\text { 0156. } 10 \\
1967 \\
32 / 17\end{array}$ \\
\hline$\ldots$ & 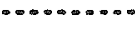 & $\Rightarrow$ & 000 & Dosos & . & $\ldots$ & 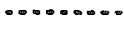 \\
\hline 1996 & 575 & & & & & 575 & 441 \\
\hline 1997 & 574 & & & & & 574 & 127 \\
\hline 1996 & & 10 & 322 & 107 & & 439 & 317 \\
\hline 1999 & & 10 & 322 & 107 & & 439 & 300 \\
\hline 2000 & & 10 & 32 & 107 & & 439 & 299 \\
\hline 2001 & & 10 & 322 & 107 & & 439 & 290 \\
\hline 2002 & & 10 & 32 & 107 & & 439 & 282 \\
\hline 2003 & & 10 & 322 & 107 & & 439 & 279 \\
\hline 2004 & & 10 & 322 & 107 & & 439 & 266 \\
\hline 2005 & & 10 & 322 & 107 & & 439 & 258 \\
\hline 2006 & & 10 & 322 & 107 & & 439 & 250 \\
\hline 2007 & & 10 & 322 & 107 & & 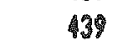 & 243 \\
\hline 2006 & & 10 & 32 & 107 & & 433 & 236 \\
\hline 3009 & & 10 & 322 & 107 & & 439 & 229 \\
\hline 2010 & & 11 & 322 & 107 & & 439 & 222 \\
\hline 2011 & & 10 & 322 & 107 & & 439 & 218 \\
\hline 202 & & 10 & 32 & 107 & & 439 & 210 \\
\hline 2013 & & 10 & 322 & 107 & & 439 & 209 \\
\hline 2014 & & 10 & 322 & 107 & & 439 & 198 \\
\hline 2015 & & 10 & 322 & 107 & & 139 & 192 \\
\hline 2016 & & 10 & 322 & 107 & & 439 & 18 \\
\hline 2087 & & 10 & 322 & 107 & & 439 & 111 \\
\hline 2018 & & 10 & 322 & 107 & & 339 & 176 \\
\hline 2019 & & 10 & 322 & 107 & & 439 & 170 \\
\hline 2020 & & 10 & 322 & 109 & & 439 & 168 \\
\hline 2021 & & 10 & 322 & 107 & & 439 & 161 \\
\hline 2022 & & 10 & 322 & 107 & & 439 & 156 \\
\hline 2023 & & & & & 0 & & \\
\hline POTÂL & 1.1989 & 250 & 0.150 & 2,475 & 0 & 12,124 & 6,556 \\
\hline & 86 & 130 & 4172 & 1,396 & 0 & 6,556 & \\
\hline
\end{tabular}

QUANITIIES (ITU)

AMMUAL OUAM DISC

OUAN. 3\%/YR

SHPPED TO 1818

20 T0TAL

OSC COS!

$122 \quad 2 \% \quad 21 \% \quad 0 \% \quad 10 \%$

HII COST

4.99 
TARLE D-13

CASE IVC - COST OF IRAMSPORI OF SPEMT PUEL FROM REACTORS TO DOE PACILITIES

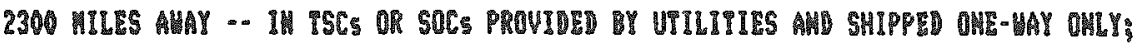

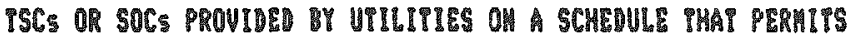

DOE AUTDAHCE OF PURCHASE OP A TRAMSPORT CAST

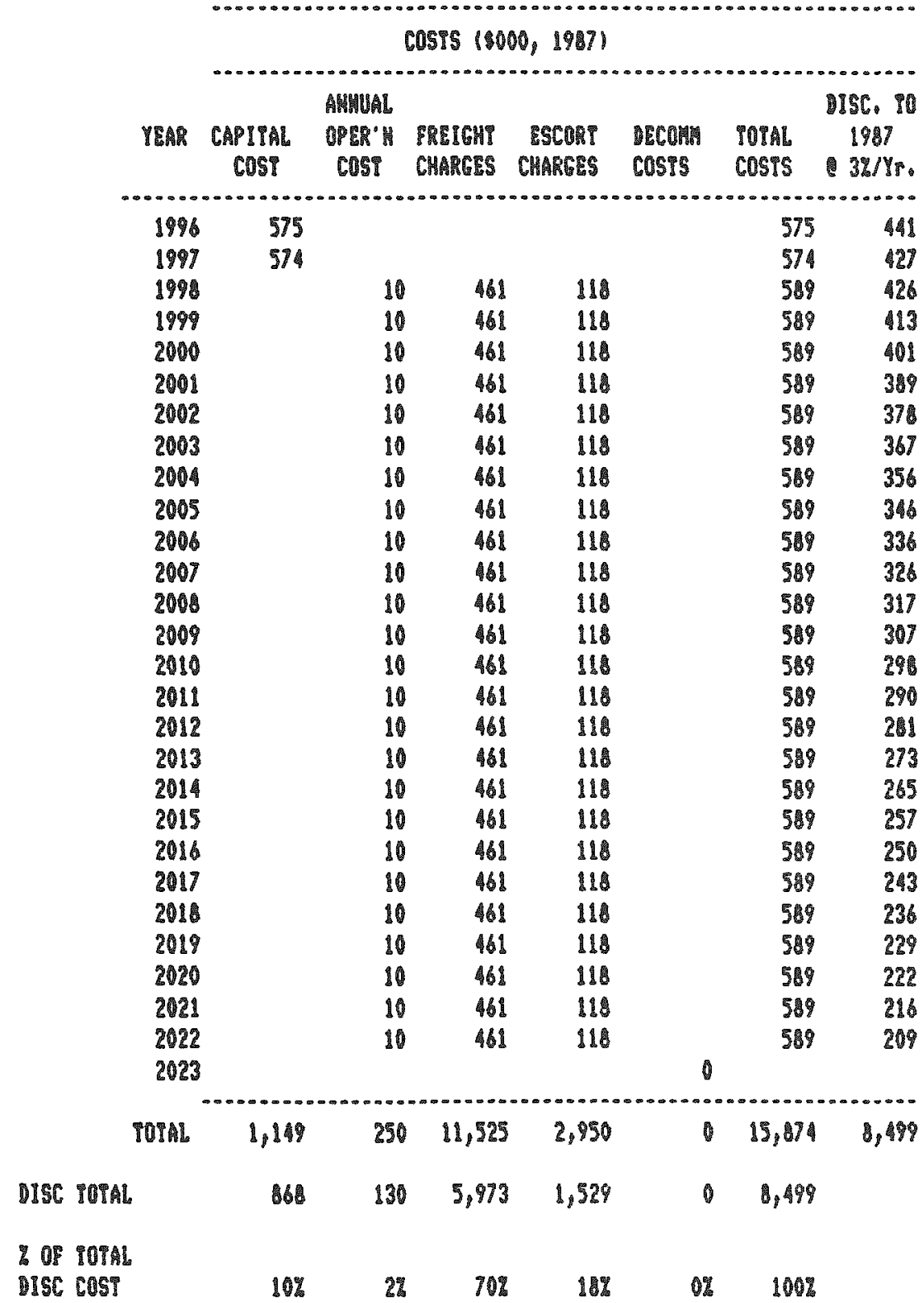

QUATITES (MTU)

AMHUAL OUAN DISC

QUA\%. $3 \% / Y$

SHIPPED 101987

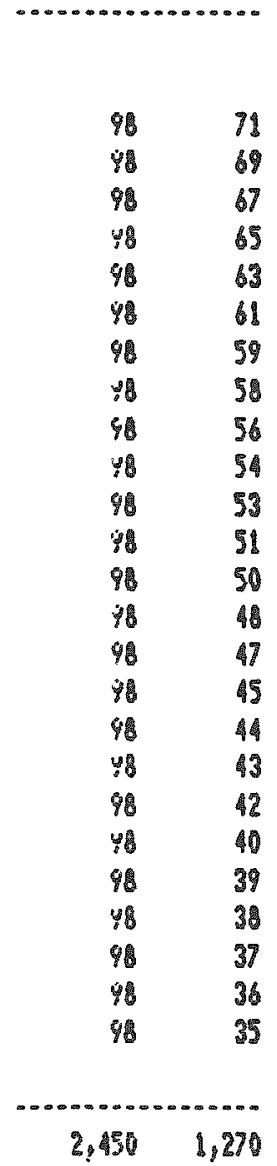

MISC COST

10\% 2\% $70 \%$ 19\%

UMIT COST

6.69 
TABLE D-14

CASE UA - COST OF IRAMSPORT OR SPENI PUEL PROM XEACTORS TO DOR FACILITIES

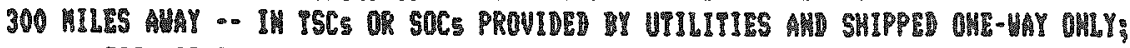

PSCS OR SOCS PROUIDED UY UILITIES OH A SCHEUULE THA DOES MOT PERII

DOE AUDIDAMCE OF PURCHASE OF A IRAMSPORI CASE

\begin{tabular}{|c|c|c|c|c|c|c|c|}
\hline \multicolumn{8}{|c|}{ COSTS $(10000,197)$} \\
\hline YEAR & $\begin{array}{c}\text { CAPIPAL } \\
\text { COSP }\end{array}$ & $\begin{array}{c}\text { AMHUAL } \\
\text { OPER } \\
\text { COST }\end{array}$ & $\begin{array}{l}\text { PREIGI } \\
\text { CMARGES }\end{array}$ & $\begin{array}{r}\text { FSCOET } \\
\text { CHARGES }\end{array}$ & $\begin{array}{l}\text { DEOH:H } \\
\text { Cos!s }\end{array}$ & $\begin{array}{l}\text { TOPAL } \\
\text { COSTS }\end{array}$ & $\begin{array}{c}\text { 015C. I0 } \\
1987 \\
32 / 17\end{array}$ \\
\hline 1996 & 1,037 & & & & & 1,037 & 795 \\
\hline 1997 & 1,036 & & & & & 1036 & 771 \\
\hline 199 & & 10 & 253 & 100 & & 363 & 262 \\
\hline 1999 & & 10 & 253 & 100 & & 363 & 255 \\
\hline 2000 & & 10 & 253 & 100 & & 363 & 247 \\
\hline 2001 & & 10 & 253 & 100 & & 363 & 290 \\
\hline 2002 & & 10 & 253 & 100 & & 363 & 233 \\
\hline 2003 & & 10 & 253 & 100 & & 363 & 226 \\
\hline 2004 & & 10 & 253 & 100 & & 363 & 220 \\
\hline 2005 & & 10 & 253 & 100 & & 363 & 213 \\
\hline 2006 & & 10 & 253 & 100 & & 363 & 207 \\
\hline 2007 & & 10 & 253 & 100 & & 363 & 201 \\
\hline 200 & & 10 & 253 & 100 & & 363 & 195 \\
\hline 2009 & & 10 & 253 & 100 & & 383 & 199 \\
\hline 2010 & & 10 & 253 & 100 & & 363 & 104 \\
\hline 2011 & & 10 & 253 & 100 & & 363 & 179 \\
\hline 2012 & & 10 & 253 & 100 & & 363 & 173 \\
\hline 2013 & & 10 & 253 & 100 & & 363 & 168 \\
\hline 2011 & & 10 & 253 & 100 & & 363 & 163 \\
\hline 2015 & & 10 & 253 & 100 & & 363 & 159 \\
\hline 2016 & & 10 & 253 & 100 & & 363 & 154 \\
\hline 2017 & & 10 & 253 & 100 & & 363 & 150 \\
\hline 2016 & & 10 & 253 & 100 & & 363 & 195 \\
\hline 2017 & & 10 & 253 & 100 & & 363 & 191 \\
\hline 2020 & & 10 & 253 & 100 & & 363 & 137 \\
\hline 2021 & & 10 & 253 & 100 & & 363 & 133 \\
\hline 2022 & & 10 & 253 & 100 & & 363 & 129 \\
\hline 2023 & & & & & 0 & & \\
\hline T0TAL & 2,073 & 250 & 6,325 & 2,500 & 0 & 11,146 & 6,289 \\
\hline & 1,566 & 130 & 3,270 & 1,298 & 0 & 6,269 & \\
\hline
\end{tabular}

DUAMTITIE (MVU)

AMMUAL MUAH DISC

QuAh. $3 \mathrm{~g} / \mathrm{M}$

WIPPED 1097

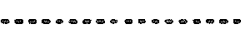

$176 \quad 127$

$176 \quad 123$

$176 \quad 120$

176116

176413

$176 \quad 110$

$176 \quad 106$

17103

$176 \quad 100$

$176 \quad 97$

$176 \quad 95$

$176 \quad 92$

17689

175

176

$176 \quad 82$

17879

1787

$176 \quad 75$

17673

17670

$176 \quad 86$

176

$176 \quad 64$

196

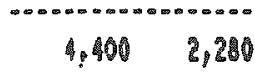

20\% TOTAL

DISC COST

$25 \% \quad 2 \% \quad 52 \% \quad 21 \% \quad 0 \% \quad 100 \%$

WhII cos? 2.75 
TABLP D-15

CASE UD - COST OF TRAMSPORT OP SPEMI PUEL FROA ARACTORS PO DOE FACLLITES

900 HILES AUAY - IIN ISCE OR SOCS PRCUIDED WY UTILITIES AMD SHIPPED OHE-WAY OHLY

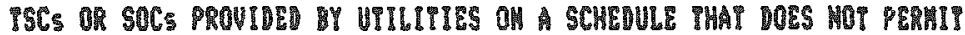
DOR AVOIDANCE OF PURCHASE OF A IRAMSPORT CAST

\begin{tabular}{|c|c|c|c|c|c|c|c|}
\hline $\mathrm{YEAR}$ & $\begin{array}{c}\text { CAPIIIAL } \\
\text { COSP }\end{array}$ & $\begin{array}{l}\text { AMULAL } \\
\text { OPER ' H } \\
\text { COST }\end{array}$ & $\begin{array}{l}\text { PREICHI } \\
\text { CHARGES }\end{array}$ & $\begin{array}{l}\text { ESCONT } \\
\text { CHARGES }\end{array}$ & $\begin{array}{l}\text { DEcom } \\
\text { COSTS }\end{array}$ & $\begin{array}{l}\text { TOEAL } \\
\text { COSIS }\end{array}$ & $\begin{array}{c}D 15 C_{8} 10 \\
1997 \\
3 \% / Y \mathrm{~T} .\end{array}$ \\
\hline 1996 & 1,037 & & & & & 1,037 & 795 \\
\hline 1997 & 1,036 & & & & & 1,036 & 77 \\
\hline 1998 & & 10 & 322 & 107 & & 139 & 31 \\
\hline 1899 & & 10 & 322 & 107 & & 439 & 36 \\
\hline 2000 & & 10 & 322 & 107 & & 439 & 299 \\
\hline 2001 & & 10 & 32 & 107 & & 439 & 290 \\
\hline 2002 & & 10 & 32 & 107 & & 439 & 282 \\
\hline 2003 & & 10 & 32 & 107 & & 439 & 274 \\
\hline 2004 & & 10 & 322 & 107 & & 439 & 266 \\
\hline 2005 & & 10 & 322 & 107 & & 439 & 258 \\
\hline 2006 & & 10 & 322 & 107 & & 439 & 250 \\
\hline 20097 & & 10 & 322 & 107 & & 439 & 243 \\
\hline 2006 & & 10 & 322 & 107 & & 439 & 236 \\
\hline 2009 & & 10 & 322 & 107 & & 439 & 229 \\
\hline 2010 & & 10 & 322 & 107 & & 439 & 222 \\
\hline 2011 & & 10 & 122 & 107 & & 139 & 21 \\
\hline 2012 & & 10 & 322 & 107 & & 439 & $y$ \\
\hline 2013 & & 10 & 322 & 107 & & 439 & 204 \\
\hline 2014 & & 10 & 322 & 107 & & 439 & 15 \\
\hline 2015 & & 10 & 322 & 1007 & & 439 & 19 \\
\hline 2016 & & 10 & 322 & 107 & & 439 & 186 \\
\hline 2017 & & 10 & 322 & 107 & & 439 & 181 \\
\hline 2018 & & 10 & วิ2ใ & 107 & & 439 & 17 \\
\hline 2018 & & 10 & 322 & 107 & & 139 & 170 \\
\hline 2020 & & 10 & 322 & 107 & & 439 & 16 \\
\hline 2021 & & 10 & 322 & 107 & & 439 & 16 \\
\hline 2022 & & 10 & 322 & 107 & & 439 & 15 \\
\hline 2023 & & & & & 0 & & \\
\hline TOTAR & 2,073 & 250 & 1,050 & 2,675 & 0 & 13,048 & 254 \\
\hline
\end{tabular}

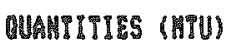

AMMUAL QUAM DISC

QUAM. 3\%/YR

SHIPPED IO 1987

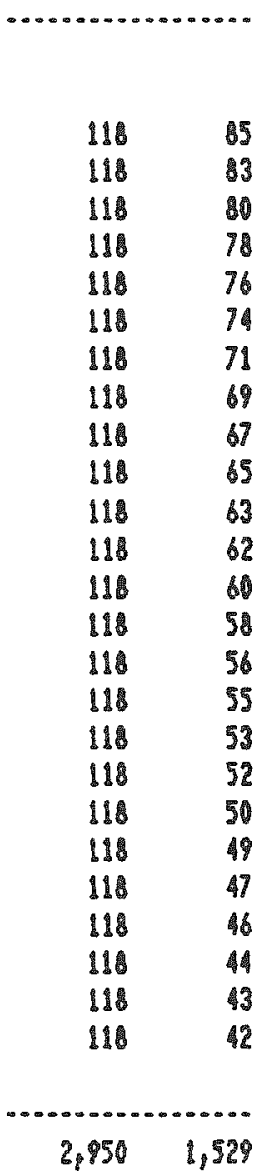

DISC POTAL

$1,566 \quad 130 \quad 4,172 \quad 1,388$

7,254

20 OP TOTh

DISC COSI

$22 \% \quad 2 \% \quad 59 \% \quad 19 \%$ or $100 \%$

UมIT $\cos \quad 4.74$ 
TADLE D-16

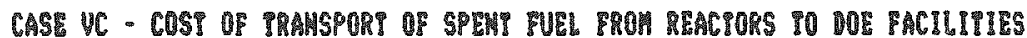

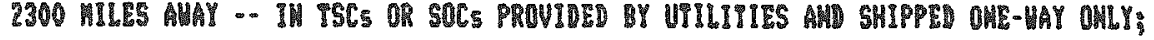
TSG OR SOCS PROUIDED WY UTLLIIIES OH A SCHEULE THAI DOES HOT PERIT DOE AUOITAMCE OF PURCHASE OF $A$ TRAMSPORI CASE

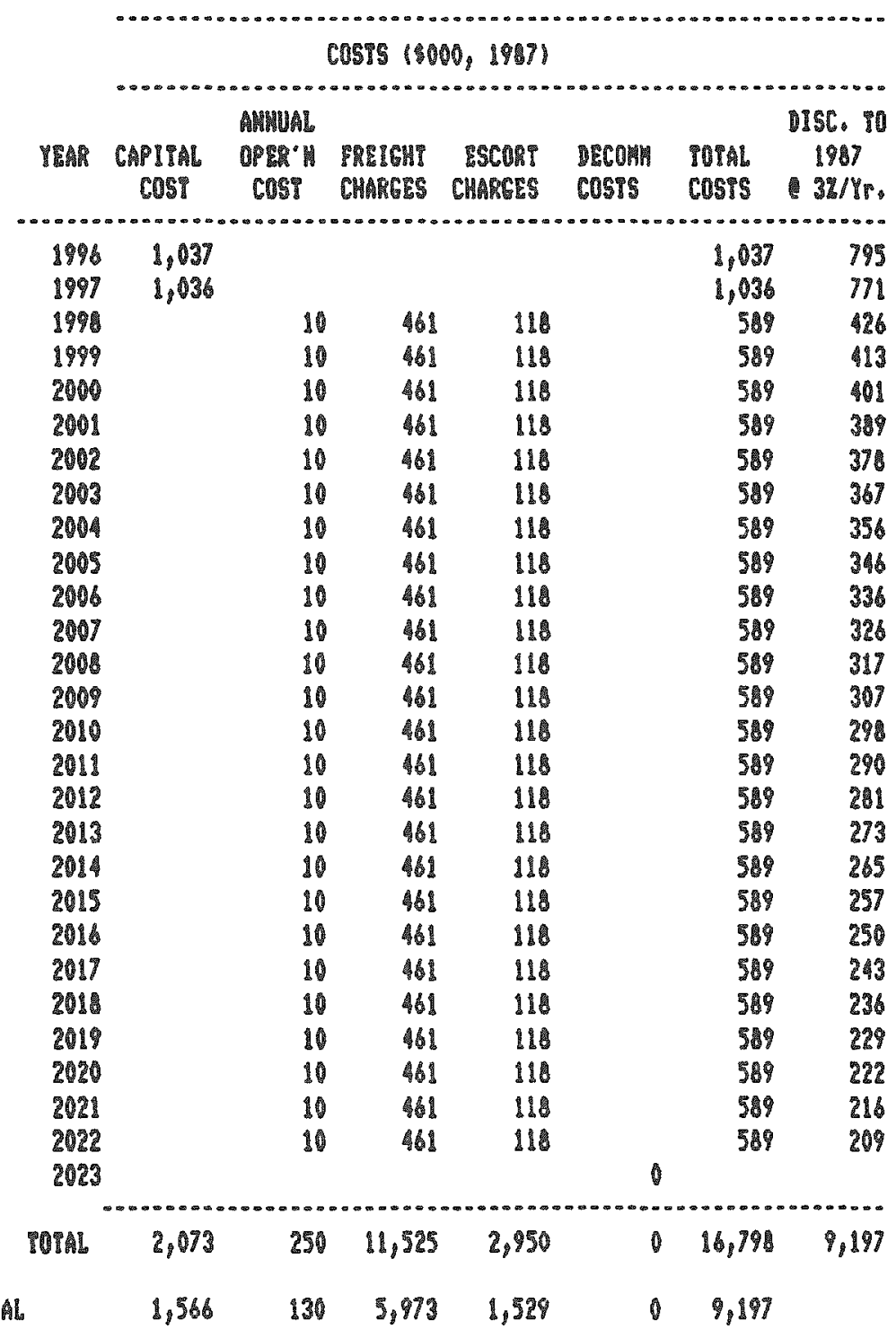

MUAKITIES (MTU)

AHUUL QUAM ISC

טuar. 3W/YR

SHIPPED 101987

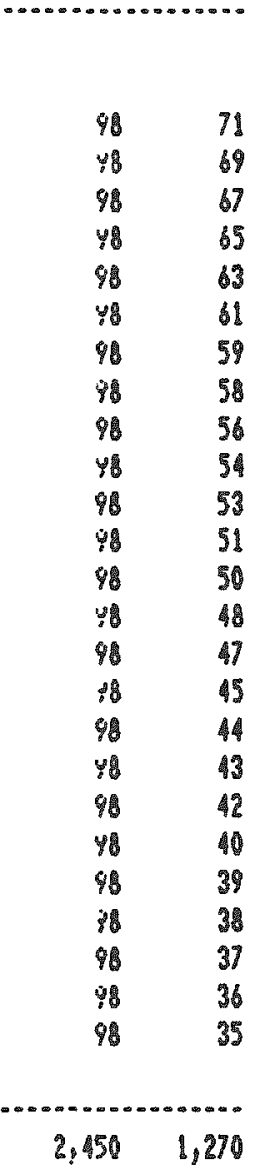

MISC TOTh

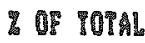

DISC COST

$17 \% \quad 12 \quad 65 \% \quad 17 \% \quad 0 \% \quad 100 \%$

UแI CoS

7.24 
TABLE D-17

CASE VIA - COST OF TRAMSPORI OF SPPUI PUEL BROH REACTORS IO DOE PACILIYLS 300 MILES AWAY - IN SOCS PROUIDED BY UTLLITES IN OUERPACY AHD SHIPPED ONE-WAY ONLY SOCE PROUIDED BY UTILITIES OH A SCHEDULE PHAT PERMITS DOE AVOIDAMCE OF PURCHASE OF A IRANSPORI CAST

\begin{tabular}{|c|c|c|c|c|c|c|c|}
\hline & & & COSTS 1300 & 00,19871 & & & \\
\hline YThA & $\begin{array}{c}\text { CAPIPALL } \\
\text { COST }\end{array}$ & $\begin{array}{l}\text { AMUUAAL } \\
\text { OPER'I } \\
\text { COST }\end{array}$ & $\begin{array}{l}\text { PREICH } \\
\text { CHARGES }\end{array}$ & $\begin{array}{l}\text { ESCOE" } \\
\text { CMARGES }\end{array}$ & $\begin{array}{l}\text { DECOMA } \\
\text { Cosis }\end{array}$ & $\begin{array}{l}\text { TOIAL } \\
\text { COSPS }\end{array}$ & $\begin{array}{l}\text { MISC. } 30 \\
1987 \\
3 \% / Y \text {. }\end{array}$ \\
\hline & & & & & & & \\
\hline $\begin{array}{l}1996 \\
1997\end{array}$ & $\begin{array}{l}865 \\
884\end{array}$ & & & & & $\begin{array}{l}865 \\
834\end{array}$ & 643 \\
\hline 1998 & & 35 & 395 & 100 & & 530 & 363 \\
\hline 1999 & & 35 & 395 & 100 & & 530 & 372 \\
\hline 2000 & & 35 & 395 & 100 & & 530 & 361 \\
\hline 2001 & & 35 & 395 & 100 & & 530 & 350 \\
\hline 2002 & & 35 & 395 & 100 & & 530 & 340 \\
\hline 2003 & & 35 & 395 & 100 & & 530 & 330 \\
\hline 2004 & & 35 & 395 & 100 & & 530 & 321 \\
\hline 2005 & & 35 & 395 & 100 & & 530 & 311 \\
\hline 2006 & & 35 & 395 & 100 & & 530 & 302 \\
\hline 2007 & & 35 & 395 & 100 & & 530 & 293 \\
\hline 2008 & & 35 & 395 & 100 & & 530 & 285 \\
\hline 2009 & & 35 & 395 & 100 & & 530 & 277 \\
\hline 2010 & & 35 & 395 & 100 & & 530 & 269 \\
\hline 2011 & & 35 & 395 & 100 & & 530 & 261 \\
\hline 2012 & & 35 & 385 & 100 & & $\$ 30$ & 253 \\
\hline 2013 & & 35 & 395 & 100 & & 530 & 246 \\
\hline 2014 & & 35 & 395 & 190 & & 530 & 239 \\
\hline 2015 & & 35 & 395 & 100 & & 530 & 232 \\
\hline 2016 & & 35 & 395 & 100 & & 530 & 225 \\
\hline 2017 & & 35 & 395 & 100 & & 530 & 218 \\
\hline 2018 & & 35 & 395 & 100 & & 530 & 212 \\
\hline 2019 & & 35 & 395 & 100 & & 530 & 206 \\
\hline 2020 & & 35 & 395 & 100 & & 530 & 200 \\
\hline 2021 & & 35 & 395 & 100 & & 530 & 194 \\
\hline 2022 & & 35 & 395 & 100 & & 530 & 188 \\
\hline 2023 & & & & & 27 & 27 & 9 \\
\hline TOPAL & 1,729 & 875 & 9,875 & 2,500 & 27 & 15,006 & 0,182 \\
\hline
\end{tabular}

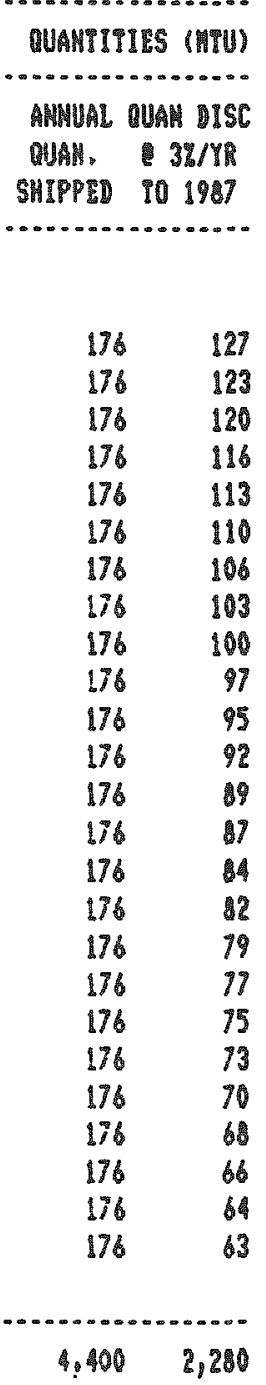

DISC POTAL

$\begin{array}{llllll}1,306 & 453 & 5,118 & 1,296 \quad 9,182\end{array}$

\% OF IOTAL

DISC COS"

$16 \% \quad 6 \% \quad 63 \% \quad 18 \% \quad 0 \% \quad 100 \%$

UHIT COST

3.59 
TABLE D.16

CASE UID - COSY OR IRAMSPOR OF SPEWT FUEL PROM REACTORS TO DOE FACHLITIES

900 WILES AWAY -- IN SOCS PROVIDED BY UTILITES IN OUERPACX AND SHIPPED ONP-WAY OWLY SOCS PROUIDED BY UTILITIES OM A SCHEDULE THAT PERITS DOE AVOIDANCE OF PURCHASE OF A IRAMSPORI CASI

\begin{tabular}{|c|c|c|c|c|c|c|c|}
\hline YEAR & $\begin{array}{c}\text { CAPITAL } \\
\text { COSI }\end{array}$ & $\begin{array}{l}\text { AMWUAL } \\
\text { OPER' } \\
\text { COST }\end{array}$ & $\begin{array}{l}\text { FREIGHT } \\
\text { CHARGES }\end{array}$ & $\begin{array}{l}\text { ESCORI } \\
\text { CHASGES }\end{array}$ & $\begin{array}{l}\text { DECOMH } \\
\text { COSPS }\end{array}$ & $\begin{array}{l}\text { TOTAL } \\
\text { costs }\end{array}$ & $\begin{array}{c}\text { DISC, } 10 \\
1987 \\
3 \% / Y \mathrm{P}\end{array}$ \\
\hline 1996 & 865 & & & & & 865 & \\
\hline 1997 & 864 & & & & & 384 & \\
\hline 199: & & 35 & 501 & 107 & & 843 & \\
\hline 1999 & & 35 & sol & 107 & & 843 & \\
\hline 2000 & & 35 & 501 & 107 & & 649 & \\
\hline 2001 & & 35 & 501 & 107 & & 843 & \\
\hline 2002 & & 35 & 501 & 107 & & 843 & \\
\hline 2003 & & 35 & 501 & 107 & & 843 & \\
\hline 2004 & & 35 & 501 & 107 & & 843 & \\
\hline 2005 & & 35 & 501 & 107 & & 843 & \\
\hline 2006 & & 35 & 501 & 107 & & 643 & \\
\hline 2007 & & 35 & 501 & 107 & & 643 & \\
\hline 2008 & & 35 & 581 & 107 & & 643 & \\
\hline 2009 & & 35 & 501 & 107 & & 643 & \\
\hline 2010 & & 35 & 501 & 107 & & 843 & \\
\hline 2011 & & 35 & 501 & 107 & & 643 & \\
\hline 2012 & & 35 & 501 & 107 & & 843 & \\
\hline 2013 & & 35 & 501 & 107 & & 843 & \\
\hline 2014 & & 35 & 501 & 107 & & 643 & \\
\hline 2015 & & 35 & 501 & 107 & & 643 & \\
\hline 2016 & & 35 & 501 & 107 & & 643 & \\
\hline 2017 & & 35 & 501 & 107 & & 643 & \\
\hline 2018 & & 35 & 501 & 107 & & 643 & \\
\hline 2019 & & 35 & 501 & 107 & & 643 & \\
\hline 2020 & & 35 & 501 & 107 & & 643 & \\
\hline 2021 & & 35 & 501 & 107 & & 843 & \\
\hline 2022 & & 35 & 501 & 107 & & 643 & \\
\hline 2023 & & & & & 27 & 27 & \\
\hline roTal & 1,729 & 875 & 12,525 & 2,675 & 27 & 17,331 & 780 \\
\hline
\end{tabular}

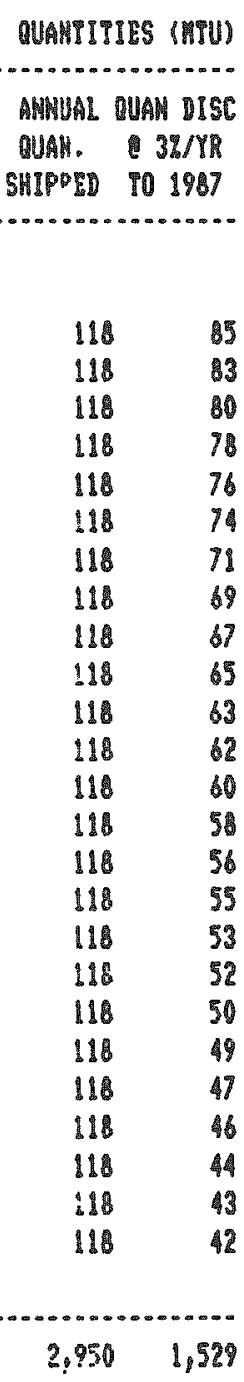

DISC POTAL $\quad 1,306 \quad 453 \quad 6,491 \quad 1,396 \quad 9 \quad 9,647$

7 OF TOTAL DISC CO5? 
TABLE D-19

CASE UIC - COST OF TRAMSPOR OF SPEMY FULL BROM REACTORS TO DOE PACILITIES

2300 HILES AHAY -. IN SOCS PROUIDED BY UTLLITES IM OUERPACK AMD SHIPPED OHE-WAY OMLY:

SOCS PROULDE BY URLITIES ON A SCHEDULE PMAT PERMITS DOE AVOIDANCE OF PURCHASE OF A IRAMSPORT CASK

\begin{tabular}{|c|c|c|c|c|c|c|c|c|c|}
\hline \multirow[b]{2}{*}{ reAR } & \multicolumn{7}{|c|}{ CosTs $(8000,1987)$} & \multicolumn{2}{|c|}{ QUANTIIIISS (ทพT) } \\
\hline & $\begin{array}{c}\text { CAPITAL } \\
\text { COST }\end{array}$ & $\begin{array}{l}\text { AMHUAL } \\
\text { OPER' } \\
\text { COST }\end{array}$ & $\begin{array}{l}\text { FRETGHT } \\
\text { CHARGES }\end{array}$ & $\begin{array}{l}\text { ESCORT } \\
\text { CHARGES }\end{array}$ & $\begin{array}{l}\text { DCOOH } \\
\text { cosTS }\end{array}$ & $\begin{array}{l}\text { TOTAL } \\
\text { COSTS }\end{array}$ & $\begin{array}{c}\text { DISC, } 10 \\
1987 \\
3 \% / V \text {. }\end{array}$ & $\begin{array}{l}\text { AMNUAL } \\
\text { QUAR. } \\
\text { SHIPPSD }\end{array}$ & $\begin{array}{l}\text { QUAM DISC } \\
\text { E } 3 \% / Y R \\
\text { 10 } 1987\end{array}$ \\
\hline 1996 & $\$ 65$ & & & & & 865 & 663 & & \\
\hline 1897 & 884 & & & & & 864 & 643 & & \\
\hline 1998 & & 35 & 717 & 118 & & 870 & 829 & 98 & 71 \\
\hline 1999 & & 35 & 717 & 118 & & 870 & 810 & 48 & 69 \\
\hline 2000 & & 35 & 717 & 118 & & 870 & 592 & 98 & 67 \\
\hline 2001 & & 35 & 717 & 118 & & 870 & 575 & 8 & 65 \\
\hline 2002 & & 35 & 717 & 118 & & 870 & 558 & 98 & 63 \\
\hline 2003 & & 35 & 717 & 118 & & 870 & 542 & 桨 & $\$ 1$ \\
\hline 2004 & & 35 & 717 & 118 & & 870 & 526 & 48 & 59 \\
\hline 2005 & & 35 & 717 & 118 & & 870 & 511 & 48 & 58 \\
\hline 2006 & & 35 & 717 & 118 & & 870 & 496 & 98 & 56 \\
\hline 2007 & & 35 & 717 & 118 & & 870 & 482 & 48 & 54 \\
\hline 2000 & & 35 & 717 & 118 & & 870 & 486 & 98 & 53 \\
\hline 2009 & & 35 & 717 & 118 & & 870 & 454 & 4 & $5 t$ \\
\hline 2010 & & 35 & 717 & 118 & & 870 & 941 & 48 & 50 \\
\hline 2011 & & 35 & 717 & 110 & & 870 & 428 & 18 & 48 \\
\hline 2012 & & 35 & 717 & 118 & & 870 & 116 & 98 & 47 \\
\hline 2013 & & 35 & 717 & 118 & & 870 & 403 & $\because 8$ & 45 \\
\hline 2014 & & 35 & 717 & 118 & & 870 & 392 & 98 & 44 \\
\hline 2015 & & 35 & 717 & 118 & & 870 & 380 & 8 & 43 \\
\hline 2016 & & 35 & 717 & 118 & & 870 & 369 & 98 & 42 \\
\hline 2017 & & 35 & 717 & 118 & & 970 & 358 & 38 & 80 \\
\hline 2016 & & 35 & 717 & 118 & & 970 & 340 & 98 & 39 \\
\hline 2019 & & 35 & 717 & 118 & & 870 & 338 & 8 & 38 \\
\hline 2020 & & 35 & 717 & 118 & & 870 & 328 & 98 & 37 \\
\hline 2021 & & 35 & 717 & 118 & & 870 & 318 & 8 & 36 \\
\hline 2022 & & 35 & 717 & 118 & & 870 & 309 & 98 & 35 \\
\hline 2023 & & & & & 27 & 27 & $\theta$ & & \\
\hline TOTAL & 1,729 & 875 & 17,925 & 2,850 & 27 & 23,506 & 12,588 & 2.450 & 1,270 \\
\hline DISC POTAL & 1,306 & 453 & 9,290 & 1,329 & 9 & 12,588 & & & \\
\hline$\%$ OP FOTAL & & & & & & & & & \\
\hline DISC COS? & $10 \%$ & $4 \%$ & 741 & $12 \%$ & $0 \%$ & $100 \%$ & & UnIT COST & 9.91 \\
\hline
\end{tabular}


PABLE D-20

CASE YISA - COST OF PRANSPORT OP SPEMT PUEL PROM REACTORS PO DOE PACILIIIES

300 HILES AMAY -. IM SOCS PKOUIDED BY UTILITES IN OUERPACC AMB SHIPPRD ONE-HAY OULY:

SOCS PROUIDED BY UTILITIES OH A SCHESULE THAT DOES NOT PEQMIP

DQE AUDIDANCE OF PURCHASE OF A TRAMSPORT CASL

\begin{tabular}{|c|c|c|c|c|c|c|c|c|c|}
\hline & $\cdots$ & 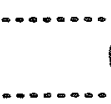 & costs 180 & $00,1987)$ & & & & OUAKIIT & IES (MTU) \\
\hline YREA & $\begin{array}{c}\text { CAPITAL } \\
\text { COST }\end{array}$ & $\begin{array}{l}\text { AMUUAL } \\
\text { OPER' } \\
\text { COS }\end{array}$ & $\begin{array}{l}\text { PREIGHP } \\
\text { CHARGES }\end{array}$ & $\begin{array}{l}\text { ESCORI } \\
\text { CHARGES }\end{array}$ & $\begin{array}{l}\text { DECOMm } \\
\text { CosTs }\end{array}$ & $\begin{array}{l}\text { POPAL } \\
\text { COSTS }\end{array}$ & $\begin{array}{c}\text { USC. } 10 \\
1967 \\
32 / \mathrm{YP}^{2}\end{array}$ & $\begin{array}{l}\text { AHUUAL } \\
\text { OUAM. } \\
\text { SHIPFED }\end{array}$ & $\begin{array}{l}\text { QUAN DISO } \\
\text { E } 3 \% / Y R \\
\text { IO } 1987\end{array}$ \\
\hline$\cdots$ & 10....... & 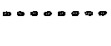 & $\cdots$ & $\cdots$ & & 10.0. & 00000000 & a.......... & $\cdots$ \\
\hline $\begin{array}{l}1996 \\
1997\end{array}$ & $\begin{array}{l}1,327 \\
1,326\end{array}$ & & & & & $\begin{array}{l}1,327 \\
1,326\end{array}$ & $\begin{array}{r}1,017 \\
987\end{array}$ & & \\
\hline 1998 & & 35 & 395 & 100 & & $\begin{array}{r}1,360 \\
330\end{array}$ & 383 & 176 & 127 \\
\hline 1999 & & 35 & 395 & 100 & & 530 & 372 & 176 & 123 \\
\hline 2000 & & 35 & 385 & 100 & & 530 & 361 & 178 & 120 \\
\hline 2001 & & 35 & 395 & 100 & & 530 & 350 & 176 & 116 \\
\hline 2002 & & 35 & 395 & 100 & & 530 & 340 & 176 & 113 \\
\hline 2003 & & 35 & 395 & 100 & & 530 & 330 & 176 & 110 \\
\hline 2004 & & 35 & 395 & 100 & & 530 & 321 & 178 & 106 \\
\hline 2005 & & 35 & 395 & 100 & & 530 & 311 & $17 \%$ & 103 \\
\hline 2006 & & 35 & 395 & 100 & & 530 & $30 ?$ & 178 & 100 \\
\hline 2007 & & 35 & 395 & 100 & & 530 & 293 & 178 & 97 \\
\hline 2008 & & 35 & 395 & 100 & & 530 & 285 & 178 & 85 \\
\hline 2009 & & 35 & 395 & 100 & & 530 & 27 & 178 & 92 \\
\hline 2010 & & 35 & 395 & 100 & & 530 & 269 & 178 & 89 \\
\hline 2011 & & 35 & 395 & 100 & & 530 & 261 & 178 & 87 \\
\hline 2012 & & 35 & 395 & 100 & & 530 & 253 & 178 & 6 \\
\hline 2013 & & 35 & 395 & 100 & & 530 & 246 & 178 & 82 \\
\hline 2014 & & 35 & 395 & 100 & & 530 & 239 & 178 & 79 \\
\hline 2015 & & 35 & 395 & 100 & & 530 & 232 & $17 \%$ & 77 \\
\hline 2016 & & 35 & 395 & 100 & & 530 & 225 & 178 & 75 \\
\hline 2017 & & 35 & 395 & 100 & & 530 & 218 & 176 & 73 \\
\hline 2018 & & 35 & 395 & 100 & & 530 & 212 & 176 & 70 \\
\hline 2019 & & 35 & 395 & 100 & & 530 & 206 & 176 & 68 \\
\hline 2020 & & 35 & 395 & 100 & & 530 & 200 & 176 & 86 \\
\hline 2021 & & 35 & 395 & 100 & & 530 & 194 & 178 & 64 \\
\hline 2022 & & 35 & 395 & 100 & & 530 & 188 & 176 & 63 \\
\hline 2023 & & & & & 27 & 27 & 9 & & \\
\hline POPAL & 2,653 & 875 & 9,975 & 2,500 & 27 & 15,930 & 8,880 & 5,400 & 2,280 \\
\hline DISC TOPAL & 2,004 & 453 & 5,118 & 1,296 & 9 & 8,880 & & & \\
\hline & & & & & & & & & \\
\hline UISC $\cos T$ & $23 \%$ & $5 \%$ & $58 \%$ & $15 \%$ & $0 \%$ & $100 \%$ & & WHIT cost & 3.89 \\
\hline
\end{tabular}


TAMLE D-21

CASE UIIB - COST OF TRAMSPORT OF SPEMT FURL FROH REACFORS PO DOR FACILITIES

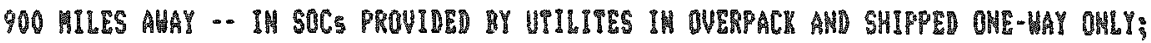
SOCE PROUIEE BY UTILITES ON A SCHEDULE PHAT DOES MOT PERHIT

DOE AWOIDAMLE OF PURCHASE OF A TRAMSPOR? CASE

\begin{tabular}{|c|c|c|c|c|c|c|c|}
\hline \multirow[b]{2}{*}{ YEA } & \multicolumn{6}{|c|}{ Cosis $(8000,1987)$} & \\
\hline & $\begin{array}{c}\text { CAPITAL } \\
\text { COST }\end{array}$ & $\begin{array}{l}\text { AMHUAL } \\
\text { OPER' } \\
\text { COSP }\end{array}$ & $\begin{array}{l}\text { BREIGHY } \\
\text { CHARGESS }\end{array}$ & $\begin{array}{l}\text { Escont } \\
\text { CHargers }\end{array}$ & $\begin{array}{l}\text { Deconth } \\
\text { costs }\end{array}$ & $\begin{array}{l}\text { TOTAL } \\
\text { COSTS }\end{array}$ & 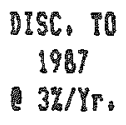 \\
\hline 1996 & 1,327 & & & & & 1,327 & 1,017 \\
\hline 1997 & 1,326 & & & & & 1,326 & 987 \\
\hline 1990 & & 35 & 501 & 107 & & 643 & 465 \\
\hline 1999 & & 35 & 501 & 107 & & 643 & 151 \\
\hline 2000 & & 35 & 501 & 107 & & 643 & 438 \\
\hline 2001 & & 35 & 501 & 107 & & 643 & 425 \\
\hline 2002 & & 35 & 501 & 107 & & 643 & 413 \\
\hline 2003 & & 35 & 501 & 107 & & 643 & 401 \\
\hline 2004 & & 35 & 501 & 107 & & 643 & 309 \\
\hline 2005 & & 35 & 501 & 107 & & 643 & 378 \\
\hline 2006 & & 35 & 501 & 107 & & 643 & 367 \\
\hline 2007 & & 35 & 501 & 107 & & 843 & 356 \\
\hline 2005 & & 35 & 501 & 107 & & 643 & 346 \\
\hline 2006 & & 35 & 501 & 107 & & 643 & 396 \\
\hline 2010 & & 35 & 501 & 107 & & 643 & 326 \\
\hline 2011 & & 35 & 501 & 107 & & 643 & 316 \\
\hline 2012 & & 35 & 501 & 107 & & 643 & 307 \\
\hline 2013 & & 35 & 501 & 107 & & 843 & 298 \\
\hline 2014 & & 35 & 501 & 107 & & 643 & 289 \\
\hline 2015 & & 35 & 501 & 107 & & 643 & 281 \\
\hline 2016 & & 35 & 501 & 107 & & 643 & 273 \\
\hline 2017 & & 35 & 501 & 107 & & 343 & 265 \\
\hline 2018 & & 35 & 501 & 107 & & 643 & 257 \\
\hline 2019 & & 35 & 501 & 107 & & 843 & 250 \\
\hline 2020 & & 35 & 501 & 107 & & 643 & 242 \\
\hline 2021 & & 35 & 501 & 107 & & 843 & 235 \\
\hline 2022 & & 35 & 501 & 107 & & 643 & 229 \\
\hline 2023 & & & & & 27 & 27 & 9 \\
\hline TOTA & 2,653 & 875 & 12,525 & 2,679 & 27 & 18,755 & 10,344 \\
\hline
\end{tabular}

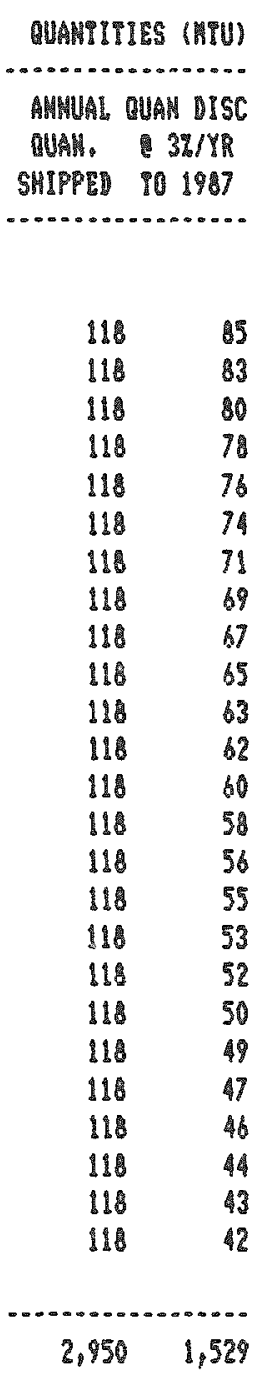

DISE TOEAL

$2,004 \quad 453 \quad 6,491 \quad 1,386 \quad 9 \quad 10,344$

6) OP IOTAL DISC COST 
TABLED D -22

CASE VIIC - COST OP TRAMSPORT OF SPEMT FUEL PROM REACTORS TO DOE FACILITIES

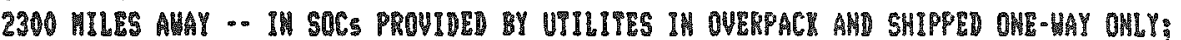

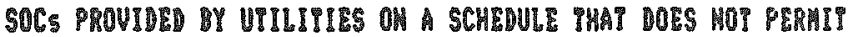

DOE AUOIDAMCE OF PURCHASE OF A TRAKSPORT CASY

\begin{tabular}{|c|c|c|c|c|c|c|c|}
\hline \multirow[b]{2}{*}{ YEAR } & \multicolumn{6}{|c|}{$\cos 15(1000,1987)$} & \multirow[b]{2}{*}{$\begin{array}{c}\text { DSE. } 10 \\
1997 \\
3 \% / 15 .\end{array}$} \\
\hline & $\begin{array}{c}\text { CAPIIIL } \\
\text { CosI }\end{array}$ & $\begin{array}{c}\text { AH以UAL } \\
\text { OPER } \\
\text { COET }\end{array}$ & $\begin{array}{l}\text { PREIGHI } \\
\text { CHARESS }\end{array}$ & $\begin{array}{r}\text { ESCORT } \\
\text { CHARES }\end{array}$ & 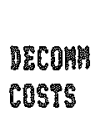 & $\begin{array}{l}\text { I0IAL } \\
\text { costs }\end{array}$ & \\
\hline 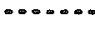 & $\ldots$ & & $\infty$ & $\infty$ & & $\cdots$ & cososos \\
\hline 1996 & 1,327 & & & & & 1,327 & 1,017 \\
\hline 1997 & 1,326 & & & & & 1,326 & 987 \\
\hline 1998 & & 35 & 717 & 110 & & 870 & 829 \\
\hline 1999 & & 35 & 717 & 118 & & 870 & 810 \\
\hline 2000 & & 35 & 717 & 110 & & 870 & 592 \\
\hline 2001 & & 35 & 717 & 118 & & 870 & 575 \\
\hline 2002 & & 35 & 917 & 110 & & 870 & 550 \\
\hline 2003 & & 35 & 717 & 118 & & 870 & 542 \\
\hline 2004 & & 35 & 717 & 116 & & 870 & 526 \\
\hline 2005 & & 35 & 717 & 116 & & 870 & 511 \\
\hline 2006 & & 35 & 717 & 110 & & 870 & 496 \\
\hline 2007 & & 35 & 717 & 118 & & 870 & 482 \\
\hline 2008 & & 35 & 717 & 110 & & 870 & 468 \\
\hline 2009 & & 35 & 717 & 118 & & 870 & 454 \\
\hline 2010 & & 35 & 717 & 110 & & 870 & 441 \\
\hline 2011 & & 35 & 717 & 118 & & 870 & 428 \\
\hline 2012 & & 35 & 717 & 110 & & 970 & 916 \\
\hline 2013 & & 35 & 717 & 110 & & 870 & .03 \\
\hline 2014 & & 35 & 717 & 118 & & 870 & 392 \\
\hline 2015 & & 35 & 717 & 118 & & 870 & 380 \\
\hline 2016 & & 35 & 717 & 116 & & 870 & 369 \\
\hline 2017 & & 35 & 717 & 110 & & 870 & 350 \\
\hline 2018 & & 35 & 717 & 118 & & 870 & 348 \\
\hline 2019 & & 35 & 717 & 110 & & 870 & 338 \\
\hline 2020 & & 35 & 717 & 111 & & 870 & 328 \\
\hline 2021 & & 35 & 717 & 110 & & 870 & 318 \\
\hline 2022 & & 35 & 717 & 118 & & 870 & 309 \\
\hline 2023 & & & & & 27 & 27 & 9 \\
\hline POTAL & 2,853 & 875 & 17,925 & 2,950 & 27 & 24,430 & 13,286 \\
\hline
\end{tabular}

GUAHTIIISE (MTU)

AMMUAL DUAM DISC

WUAH. $3 \% / \%$

SHIPED TO 1987

\begin{tabular}{|c|}
\hline 98 \\
\hline 98 \\
\hline 96 \\
\hline 98 \\
\hline 96 \\
\hline 98 \\
\hline 98 \\
\hline 98 \\
\hline 98 \\
\hline 98 \\
\hline 98 \\
\hline จิ \\
\hline 90 \\
\hline 90 \\
\hline 98 \\
\hline 98 \\
\hline 9 \\
\hline 96 \\
\hline 98 \\
\hline 9 \\
\hline 98 \\
\hline 18 \\
\hline 90 \\
\hline 98 \\
\hline 86 \\
\hline
\end{tabular}

$2,450 \quad 1,270$

DISE TUPAL

$2,004 \quad 453 \quad 9,290 \quad 1,529 \quad 13,266$

OOE TOTAL

DISC COST

$\begin{array}{llllll}15 \% & 32 & 70 \% & 12 \% & 0 \% & 100 \%\end{array}$

UNIT COST 10.46 
TABLE D-23

SUMMARY OF SPENT FUEL TRANSPORT COSTS

(1987 Dollars)

\section{Cask Scenario}

Case I - DOE-Supplied Transport Cask

Case II - TSC Used in Repetitive Shipments: DOE Avoids Purchase of Transport Cask

Case III - TSC Used in Repetitive Shipments: But DOE Does Not Avoid Purchase of Transport Cask

Case IV - TSC or SOC Used in One-Way Shipment: DOE Avoids Purchase of Transport Cask

Case V - TSC or SOC Used in One-Way Shipment: But DOE Does Not Avoid Purchase of Transport Cask

Case VI - Overpacked SOC Used in One-Way Shipment; DOE Avoids Purchase of Transport Cask

Case VII - Overpacked SOC Used in One-Way Shipment: But DOE Does Not Avoid Purchase of Transport Cask

\begin{tabular}{|c|c|c|}
\hline \multicolumn{3}{|c|}{$\begin{array}{l}\text { Unit cost }(\$ / \mathrm{kgU})^{\mathrm{b}} \text { for } \\
\text { Transport for Indicated Distances } c, d\end{array}$} \\
\hline 300 Miles & 900 Miles & 2300 miles \\
\hline$\$ 3.91$ & $\$ 6.83$ & $\$ 10.61$ \\
\hline $3.60(0.31)$ & $6.36(0.47)$ & $10.04(0.57)$ \\
\hline $3.90(0.01)$ & $6.82(0.01)$ & $10.59(0.02)$ \\
\hline $2.44(1.47)$ & $4.29(2.54)$ & $6.69(3.92)$ \\
\hline $2.75(1.16)$ & $4.74(2.09)$ & $7.24(3.37)$ \\
\hline $3.59(0.32)$ & $6.31(0.52)$ & $9.91(0.70)$ \\
\hline $3.89(0.02)$ & $6.77(0.06)$ & $10.46(0.15)$ \\
\hline
\end{tabular}

a Intact fuel assemblies

Averaged at a $3 \% /$ year net discount rate

COne-way distances shown

d Figures in parentheses are cost savings resulting from the use of utility-furnished TSC or SOC for transport instead of DOE-supplied transport cask 
From the foregoing table it can be seen that if a TSC is supplied by a utility on a schedule that permits DOE to avoid the purchase of a standard transport cask, and DOE uses the TSC in repetitive shipments in place of the standard transport cask, savings ranging from $\$ 0.31$ to $\$ 0.57 / \mathrm{kgU}$ shipped can be realized depending on the shipping distance involved. However, if the DOE is unable to avoid purchase of a standard transport cask essentially no savings are realized.

If a number of TSCS or SOCS are supplied by utilities and are used for a one-time shipment of contained spent fuel, and the DOE gets firm commitments to receive the casks on a schedule that permits it to avoid purchasing a standard transport cask, savings ranging from $\$ 1.47$ to $\$ 3.92 / \mathrm{kgU}$ shipped can be realized depending on the shipping distance involved. Even if the SOCs are shipped in overpacks, savings ranging from $\$ 0.32$ to $\$ 0.70 / \mathrm{kgU}$ shipped can be realized depending on the shipping distance involved. However, in order for DOE to avoid purchasing a single standard transport cask in both of these scenarios it would have to receive advance commitments to deliver 12 to 21 casks/year, or 300 to 525 casks over a 25-year period. This corresponds to annual shipments of 111 to 194 MTU and total shipments of 2,775 to 4,850 MTU over the 25-year period, and means that such amounts of spent fuel would have to be stored by utilities in TSCS or SOCS in order for them to be able to effect delivery of the required number of casks. On the other hand, if DOE is unable to avoid purchase of a standard transport cask, the foregoing savings would be reduced by $\$ 0.31 / \mathrm{kgU}$ to $\$ 0.55 / \mathrm{kgU}$ for both scenarios depending on the shipping distance involved.

It should be pointed out here that the foregoing savings resulting from the use of SOCs in overpacks was based on spreading the design, development and licensing cost of overpacks over 10 units. If this estimated $\$ 2.0$-million expense were to be applied to a single overpack the cost of shipments in the overpacked SOC for cases where DOE could avoid the purchase of a standard transport cask would range from $\$ 0.28 / \mathrm{kgU}$ to $\$ 0.37 / \mathrm{kgU}$ more than shipments in a standard transport cask, and for cases where DOE could not avoid the purchase of a standard transport cask would amount to $\$ 0.58 / \mathrm{kgl}$ to $\$ 0.92 / \mathrm{kgU}$ more than shipments in a standard transport cask. 
Thus, the savings involved through the use of TSCs or SOCs for shipments of spent fuel from reactors to DOE facilities are heavily dependent both on when a utility commits to turn the storage casks over to DOE for use and the schedule on which the turnover is effected. Clearly, the DOE must have a certain sized transport cask fleet available to meet its commitments for acceptance of spent fuel from the utilities. If the TSCS or SOCs are not available soon enough to meet the needs for a transport cask fleet, the fleet will otherwise have to be acquired by DOE regardless whether or not TSCS and SOCs are ultimately used for shipment.

The savings associated with the transporting of consolidated spent fuel from the reactor to DOE facilities are about 60 percent of those shown in Table D-23 for intact fuel assemblies.

\subsection{REFERENCES FOR APPENDIX D}

(1) G. W. McNair et ai, Truck and Rail Charges for Shipping Spent Fuel and Nuclear Waste, PNL-5797, June 1986

(2) U. S. Department of Energy, Federal Interim Storage Fee Study for Civilian Spent Nuclear Fuel: A Technical and Economic Analysis, DOE/S0023 , JuTy 1983 
APPENDIX E

COST OF LAG STORAGE 
APPENDIX E

COST OF LAG STORAGE

TABLE OF CONTENTS

1.0 DESIGN DESCRIPTION

Page

2.0 CAPITAL COSTS

$E-2$

3.0 OPERATING COSTS

$E-4$

4.0 TOTAL COSTS

E-5 
APPENDIX E

COST OF LAG STORAGE

It was recognized that TSCS or SOCs that might be received from utilities could be used to provide some lag storage capacity at DOE facilities. Thus, the purpose of this Appendix was to develop an estimate of the cost of lag storage at DOE facilities, and the ralationship between lag storage capacity and the cost of lag storage -- so that an assessment could be made of the savings that would result from the use of utility-delivered TSCS or SOCs for lag storage at DOE facilities.

While the designs performed to date on MRS and repository surface facilities have included facilities for lag storage, the cost of the lag storage facilities have not generally been shown as a separate line item. Moreover, such costs are not readily extracted from the overall facility costs. Therefore, for the purposes of this study, conceptual designs were developed for three different sizes of lag storage facilities, the capital and operating costs were estimated for each, and algorithms were developed relating capital and operating costs (separately) to capacity.

Lag storage is generally considered to be a storage capacity at the interface of major operations which mitigates the impacts of interruptions in the supply of materials to, or removal from, the operation. Thus, lag storage at the front end of a DOE facility (MRS or repository) can mitigate impacts of interruptions in supply of spent fuel from reactors--and it can also provide assurance that DOE can continue to accept spent fuel in the event of an interruption in operations at the DOE facilities. Lag storage at the front end of a repository can also mitigate impacts of interruptions in supply of spent fuel from an Integral MRS Facility.

For the purposes of this study it was assumed that lag storage near the receiving end of a MRS or repository facility should be about 750 MTU in the form of intact fuel assemblies. This represents 3-months storage capacity at a $3000 \mathrm{MTU} /$ year receipt rate. However, designs were also developed for capacities of 500 MTU and 250 MTU. 


\subsection{DESIGN DESCRIPTION}

The lag storage facility was assumed to be a hot cell having an inside width of 50 feet, a 5-foot wall thickness, and a length of 187 feet for 750 MTU capacity (130 foot length for 500 MTU, and 71 foot length for 250 MTU). The hot cell was assumed to be separated into two basic compartments by a 5-foot thick slab. The lower compartment is the storage compartment and contains an array of stainless steel pipes in which the fuel is stored. Access to each pipe is through cavities in the slab, and each such cavity has a plug so that the upper compartment can be occupied by personnel when al1 plugs are in place. The upper compartment of the hot cell is open ended inasmuch as it was assumed it would probably be the conduit between the cask unloading facility and the processing activity at a DOE facility.

It was assumed that cooling of the stored fuel would be by forced air circulation. Separate ventilation and air treatment facilities were provided for the upper and lower compartments of the hot cell.

\section{$2.0 \quad$ CAPITAL COSTS}

The estimates of the capital costs of 750 MTU, 500 MTU and 250 MTU capacity lag storage facilities are shown in Table E-1. 
TABLE $E-1$

BREAKDOWN OF CAPITAL COSTS OF HOT CELL LAG STORAGE FACILITIES

$(\$ 000.1987)$

Description
Nuclear Grade Concrete
Storage Cell
Ventilation Bldg-Storage
Ventilation Bldg-Loading
Ordinary Concrete
Ventilation Bldg-Storage
Ventilation Bldg-Loading
Hot Cell Coatings
Storage Cell
Ventilation Bldg-Storage
Ventilation Bldg-Loading
Lights
Storage Cell
Ventilation Bldg-Storage
Ventilation Bldg-Loading
Shielding Windows

$$
\text { 5.' Thick }
$$

Shielded Doors Ceiling Doors

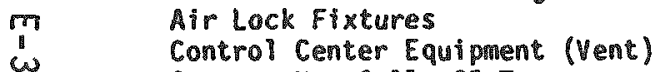

Cranes, Hot Cell, 25 Ton

Storage Modules Support

PWR

Ventilation System Equipment

Subtotal

Equipment Installation

(35\% of Bare Equipment Costs)

(15\% of Module Cost)

Subcontractor Overhead \& Profit

(45\% of Labor for Equipment Only;

Labor $50 \%$ of Installation Cost)

General Contractor Overhead Profit

(18.65\% of Equipment Only:

Included in Unit cost for others)

Engineering (15\%)

Construction Management (10\%)

Contingency (20\%)

\begin{tabular}{|c|c|c|c|c|c|c|c|}
\hline \multirow{2}{*}{\multicolumn{2}{|c|}{ Unit Cost }} & \multicolumn{2}{|c|}{$750 \mathrm{MTU}$} & \multicolumn{2}{|c|}{$500 \mathrm{MTU}$} & \multicolumn{2}{|c|}{$250 \mathrm{MTU}$} \\
\hline & & No. Units & Total & No. Units & Total & Mo. Units & Total \\
\hline$\$$ & $.55 / y d^{3}$ & $\begin{array}{r}10,928 \\
8,667 \\
1,361 \\
900 \\
561 \\
398 \\
163\end{array}$ & $\$ 22.949$ & $\begin{array}{r}7.793 \\
6.208 \\
976 \\
609 \\
340 \\
232 \\
108\end{array}$ & $\$ 16,365$ & $\begin{array}{r}4.151 \\
3,073 \\
615 \\
463 \\
199 \\
119 \\
80\end{array}$ & $\$ 8,717$ \\
\hline$\$$ & $.025 / \mathrm{ft}^{2}$ & $\begin{array}{l}93,000 \\
58,000 \\
22,000 \\
13,000\end{array}$ & 2,325 & $\begin{array}{r}65.000 \\
40,000 \\
16.000 \\
9.000\end{array}$ & 1,625 & $\begin{array}{r}39,000 \\
22,000 \\
10,000 \\
7,000\end{array}$ & 975 \\
\hline$\$$ & $.500 \mathrm{ea}$ & $\begin{array}{l}698 \\
354 \\
232 \\
112\end{array}$ & 349 & $\begin{array}{r}462 \\
240 \\
151 \\
71\end{array}$ & 231 & $\begin{array}{r}256 \\
122 \\
84 \\
50\end{array}$ & 128 \\
\hline$\$$ & $\begin{array}{r}75 \mathrm{ea} \\
50 \mathrm{ea} \\
15 \mathrm{ea} \\
3 \mathrm{ea} \\
400 \mathrm{ea}\end{array}$ & $\begin{array}{r}18 \\
16 \\
6 \\
2 \\
- \\
2\end{array}$ & $\begin{array}{r}1,350 \\
800 \\
90 \\
6 \\
250 \\
800\end{array}$ & $\begin{array}{r}12 \\
10 \\
5 \\
2 \\
5 \\
2\end{array}$ & $\begin{array}{r}900 \\
500 \\
75 \\
6 \\
175 \\
800\end{array}$ & $\begin{array}{l}6 \\
7 \\
4 \\
2 \\
= \\
2\end{array}$ & $\begin{array}{r}450 \\
350 \\
60 \\
6 \\
100 \\
800\end{array}$ \\
\hline \multirow[t]{9}{*}{$\$$} & $\begin{array}{l}8.4 \text { ea } \\
9.4 \text { ea }\end{array}$ & $\begin{array}{r}1,085 \\
683\end{array}$ & $\begin{array}{l}9,114 \\
6,420 \\
1,875 \\
\end{array}$ & $\begin{array}{l}722 \\
457\end{array}$ & $\begin{array}{l}6,065 \\
4.296 \\
1,250 \\
\end{array}$ & $\begin{array}{l}362 \\
227\end{array}$ & $\begin{array}{r}3.041 \\
2.134 \\
625 \\
\end{array}$ \\
\hline & & & 46,637 & & 32,475 & & 17,495 \\
\hline & & & $\begin{array}{l}1.932 \\
2.330\end{array}$ & & $\begin{array}{l}1.378 \\
1.554\end{array}$ & & $\begin{array}{l}882 \\
776\end{array}$ \\
\hline & & & 959 & & 660 & & 373 \\
\hline & & & 51.858 & & 36.067 & & 19.526 \\
\hline & & & 3.927 & & 2,667 & & 1,435 \\
\hline & & & 55.785 & & 38.734 & & 20,961 \\
\hline & & & $\begin{array}{r}8,368 \\
5,579 \\
69,732\end{array}$ & & $\begin{array}{r}5,810 \\
3,873 \\
48,417\end{array}$ & & $\begin{array}{r}3,144 \\
2,096 \\
26,201\end{array}$ \\
\hline & & & 13,946 & & 9,683 & & 5.240 \\
\hline \multicolumn{2}{|c|}{ Total } & & $\$ 83.678$ & & $\$ 58,100$ & & $\$ 31.441$ \\
\hline
\end{tabular}


An algorithm was developed to describe the relationship between capacity and capital cost, as follows:

$$
c_{c}=3701+113.1(x)-.00865\left(x^{2}\right)
$$

where $C_{C}$ is the capital cost of a lag storage facility having a capacity to store $x$ MTU of spent fuel. This results in an average cost of $\$ 104 / \mathrm{kgU}$ storage capacity over the 250-750 MTU capacity range (range of $\$ 100-\$ 109 / \mathrm{kgU}$ ).

\subsection{OPERATING COSTS}

The estimates of the cost of operation of the lag storage facilities described in Sections 1.0 and 2.0, above, are shown in Table E-2.

\section{TABLE E-2}

ESTIMATED ANNUAL OPERATING COST FOR LAG STORAGE FACILITIES

$$
(\$ 000,1987)
$$

\section{Description}

Labor \& Supervision

Miscellaneous Supplies

Maintenance Supplies

Electricity

Total
Cost for Indicated Capacity

$\frac{750 \text { MTU }}{\$ 1,540} \quad \frac{500 \text { MTU }}{\$ 1,255} \quad \frac{250 \text { MTU }}{\$ 960}$

210

171

131

$2,510 \quad 1,743 \quad 943$

$\begin{array}{lll}\frac{2,120}{\$ 6,380} \quad \frac{1,288}{\$ 4,457} & \frac{754}{\$ 2,788}\end{array}$

An algorithm was developed to describe the relationship between capacity and operating cost, as follows:

$$
C_{0}=1373+5.152(x)+.002\left(x^{2}\right)
$$

where $C_{0}$ is the operating cost for a lag storage facility having a capacity to store $x$ MTU of spent fuel. This results in an average cost of $\$ 7.18 / \mathrm{kgU}$ storage capacity per year over the 250-750 MTU capacity range (range of $\$ 6.20-\$ 8.20 / \mathrm{kgU})$. This amounts to about $\$ 129 / \mathrm{kgU}$ on a discounted cost basis. 
4.0 TOTAL COSTS

From the cost estimates developed in Sections 2.0 and 3.0 of this Appendix E, it is apparent that the average cost of lag storage in hot-cell type facilities is about $\$ 233 / \mathrm{kgU}$ (1987 dollars). This assumes that such facilities are operated at 100 percent capacity (fully loaded) during the lifetime thereof. In practice, this will not be the case and, therefore, the foregoing costs represent the minimum costs that can be expected to be experienced for lag storage in hot-cell type facilities. 
APPENDIX F

ESTIMATED COST FOR MRS MODULES 


\section{APPENDIX F}

ESTIMATED COST FOR MRS MODULES

TABLE OF CONTENTS

1.0 ESTIMATED COSTS

$F-1$

2.0 REFERENCES FOR APPENDIX $F$

$F-4$

$\underline{\text { Page }}$ 


\section{APPENDIX F \\ ESTIMATED COST FOR MRS MODULES}

The purpose of this Appendix is to develop an estimate of the costs and capacity for the concrete storage modules that are expected to be used at the MRS facility so that an assssment can be made of the value of a metal storage cask if it were used to substitute for a concrete module at the MRS.

\section{$1.0 \quad$ ESTIMATED COSTS}

The Raiph M. Parsons Company (Parsons) estimated the cost of a concrete storage module having a capacity for storing 16.2 MTU of consolidated rods at $\$ 220$-thousand (1985 dollars) in connection with its design of an integral MRS facility (References 1 \& 2). Parsons also estimated the cost of a concrete storage module having a capacity for storing 28.7 MTU (equivalent) of compacted structural parts at $\$ 120$-thousand (1985 dollars). The combined cost of these modules (casks) for storing consolidated fuel was $\$ 17.8 / \mathrm{kgU}$ (1985 dollars) which when escalated resulted in a 1987 cost of $\$ 18.3 / \mathrm{kgU}$. The foregoing costs do not include a component for the capital cost of the concrete module manufacturing facility.

In addition Parsons included a facility for manufacturing the concrete modules in the design of the Integral MRS facility. This facility was estimated by Parsons to cost $\$ 21.12$-million (1985 dollars) which when escalated resulted in a 1987 capital cost of $\$ 23.16-m i l l i o n$. It was recognized that if TSCS were received by DOE and used in place of some of the concrete modules that otherwise would have been used for storage, a higher cost per module would have to be allocated as a result of amortizing the $\$ 23.16$-million cost of the module manufacturing facility over a fewer number of modules.

In this connection, it was assumed that 1500 MTU/year would be received into storage for a period of 10 years $(15,000$ MTU tota 1$)$. Under these conditions the allocation of the cost of the module manufacturing facility to the cost of modules would be $\$ 1.8 / \mathrm{kgU}$, developed as follows: 


\begin{tabular}{|c|c|c|}
\hline \multirow[b]{2}{*}{ Year } & \multicolumn{2}{|c|}{ Storage Module Capacity (MTU) } \\
\hline & $\begin{array}{c}\text { Annual } \\
\text { Additions } \\
\end{array}$ & $\begin{array}{c}\text { Discounted } \\
3 \% / Y_{r} \text { to } Y_{r} 0 \\
\end{array}$ \\
\hline $\begin{array}{r}0 \\
1 \\
2 \\
3 \\
4 \\
5 \\
6 \\
7 \\
8 \\
9 \\
10\end{array}$ & $\begin{array}{l}1,500 \\
1,500 \\
1,500 \\
1,500 \\
1,500 \\
1,500 \\
1,500 \\
1,500 \\
1,500 \\
1,500 \\
\end{array}$ & $\begin{array}{l}1,456 \\
1,414 \\
1,373 \\
1,333 \\
1,294 \\
1,256 \\
1,220 \\
1,184 \\
1,150 \\
1,116 \\
\end{array}$ \\
\hline Total & 15,000 & $\underline{12,796}$ \\
\hline
\end{tabular}

Caiculations were made of the impact of reducing the number of storage modules required on the cost of the modules. The results are shown in Table F-1.

\section{TABLE F-1}

IMPACT OF REDUCING THE NUMBER OF CONCRETE MODULES REQUIRED FOR MRS ON THE COST OF THE MODULES ATTRIBUTABLE TO THE CAPITAL COST OF THE MODULE MANUFACTURING FACILITY

Reduction in Required Capacity $(\%)$

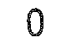

10

20

30

40

50

60

70

80

90
Cost/kgu Attributable to Concrete Storage Modules

$\$ 1.8$

2.0

2.3

2.6

3.0

3.6

4.5

6.0

9.0

18.1
Added Cost $/ \mathrm{kgU}$ for Concrete Modules Caused by Reduction in No. Modules Needed 
Thus the net savings involved in the use of TSCS delivered to DOE by a utility, as a storage module for MRS would amount to about $\$ 16.5 / \mathrm{kgU}$ stored in MRS.

In addition to the savings described above, the substitution of a TSC for a MRS concrete storage module would result in the elimination of the need to dispose of the MRS module at the end of its useful life. The TSC that replaced the module would have had to be disposed of in any event, thus the net amount of disposal costs would be reduced. A preliminary estimate was made of the cost of disposal of the MRS module, assuming that the inner metal containment vessel of the module and associated plug would be removed and packaged for disposal at a low level waste burial site, with the remainder of the concrete monolith being broken up and disposed of in a land fill. The following Table F-2 summarizes the estimated costs for disposal of MRS storage modules.

TABLE F-2

ESTIMATED COSTS FOR DISPOSAL OF MRS MODULES

(1987 Dollars)

Description

Module Handling at MRS Facility

$\frac{\text { Cost }}{\$ 1,000}$

Removal of Inner Vessel

15,120

Transport of Vessel to Barnwell

4,000

Disposal of Vessel at Barnwell

24,400

Breakup of Concrete Shell

10,080

Land and Transport to Land Fill

2,500

Disposal at Land Fill

2,000

Total

$\$ 59,100$

These costs cover disposal of MRS modules used to store consolidated fuel rods. Disposal of MRS modules used to store compacted structural parts can be expected to amount to about 55 percent of the above total $(\$ 32,500)$. 
(1) The Ralph M. Parsons Company et al, Conceptual Design Report --Integral Monitored Retrievable Storage (MRS) Facility, MRS-1, Contract No. DEAC01-84RL10436, August 1985

(2) E. R. Johnson Associates, Inc., Results of Preliminary Analysis of Differences in MRS Facility Costs Between the DOE MRS Facility Design and That Contained in the Draft JAI PRDA Report, JAI-263, October 1985. 
APPENDIX G

ESTIMATED COSTS FOR TRANSPORT OF SPENT FUEL FROM MRS TO

REPOSITORY FACILITIES IN TSCS AND STANDARD TRANSPORT CASKS 
APPENDIX G

ESTIMATED COSTS FOR TRANSPORT OF SPENT FUEL FROM MRS TO

REPOSITORY FACILITIES IN TSCS AND STANDARD TRANSPORT CASKS

TABLE OF CONTENTS

Page

1.0 CAPACITY OF CASKS FOR TRANSPORTING PACKAGES OF CONSOLIDATED G-1

FUEL

2.0 ESTIMATED COSTS FOR TRANSPORT

$G-4$

3.0 REFERENCES FOR APPENDIX G

G-8 
APPENDIX G

ESTIMATED COSTS FOR TRANSPORT OF SPENT FUEL FROM MRS TO

REPOSITORY FACILITIES IN TSCS AND STANDARD TRANSPORT CASKS

The purpose of this Appendix is to develop estimates of the cost of transporting containers of consolidated fuel from the MRS facility to a repository in ( $i$ ) standard transport casks, and ( $i i$ ) TSCs, so that the extent of the benefits involved in using TSCs could be assessed. The estimated capital and operating costs associated with the foregoing methods of shipment, and the estimated life cycle and unit costs associated with the shipment of three different packages to three different types of repositories, are developed in the following sections.

1.0 CAPACITY OF CASKS FOR TRANSPORTING PACKAGES OF CONSOLIDATED FUEL

It was recognized that consolidated spent fuel rods and compacted structural parts may be shipped from the MRS to the repository in one of basically three different forms, as follows:

(1) square cans--nominally 9-inches square

(2) sleeves--cylindrical thin-walled (0.25-inch) canisters having an outside diameter slightly smalier than the inside diameter of the repository container

(3) containers--for repository disposal.

For the purposes of this study, repository containers were assumed to have the following dimensions and capacities: 
TABLE G-1

DIMENSIONS \& CAPACITIES OF REPOSITORY CONTAINERS

Description

Outside Diameter $(\mathrm{cm})$

Inside Diameter $(\mathrm{cm})$

Length $(\mathrm{cm})$

Capacity (MTU)
Containers for

Consolidated Rods

$\frac{\text { Salt }}{87.1}$
64.5
410

5.52

\begin{tabular}{r} 
Basalt \\
\hline 54.1 \\
37.1 \\
410
\end{tabular}

1.84
Containers for

Compacted

Structural

Parts

70

67.5

410

27.5 equiv.

Either sleeves or containers will be shipped (not both), depending on whether the containerization operation is located at MRS or repository facilities. Square cans (from reactors and MRS storage) may also be shipped. However, it is assumed that in the case of tuff no sleeves will be shipped, only the tuff container, since it is already relatively thin-walled (1-inch). It is also assumed the compacted structural parts will be shipped in tuff-type containers.

For the purposes of this analysis, it was assumed that, absent any consideration of the use of TSCS for such shipments, the casks described in Reference (1) would be used to effect shipments of consolidated fuel from the MRS facility to the repository. It was recognized that there was really no "standard" DOE transport cask for this purpose, but that the cask described in Reference (1) could be expected to be reasonably representative of a "standard" cask for the purposes of this analysis. The use of TSCs for such shipments was also considered; these TSCs were assumed to be those used for reactor storage of intact fuel assemblies. An analysis was made of the capacity of TSCS for transporting cans as well as sleeves and canisters designed for the three different repository types. The results of this analysis as well as the capacities and weights of the casks described in Reference (1) are shown in Table G-2. 
TABLE G-2

CAPACITIES \& WEIGHTS OF CASKS FOR TRANSPORTING CONSOLIDATED SPENT FUEL FROM MRS TO REPOSITORY FACILITIES

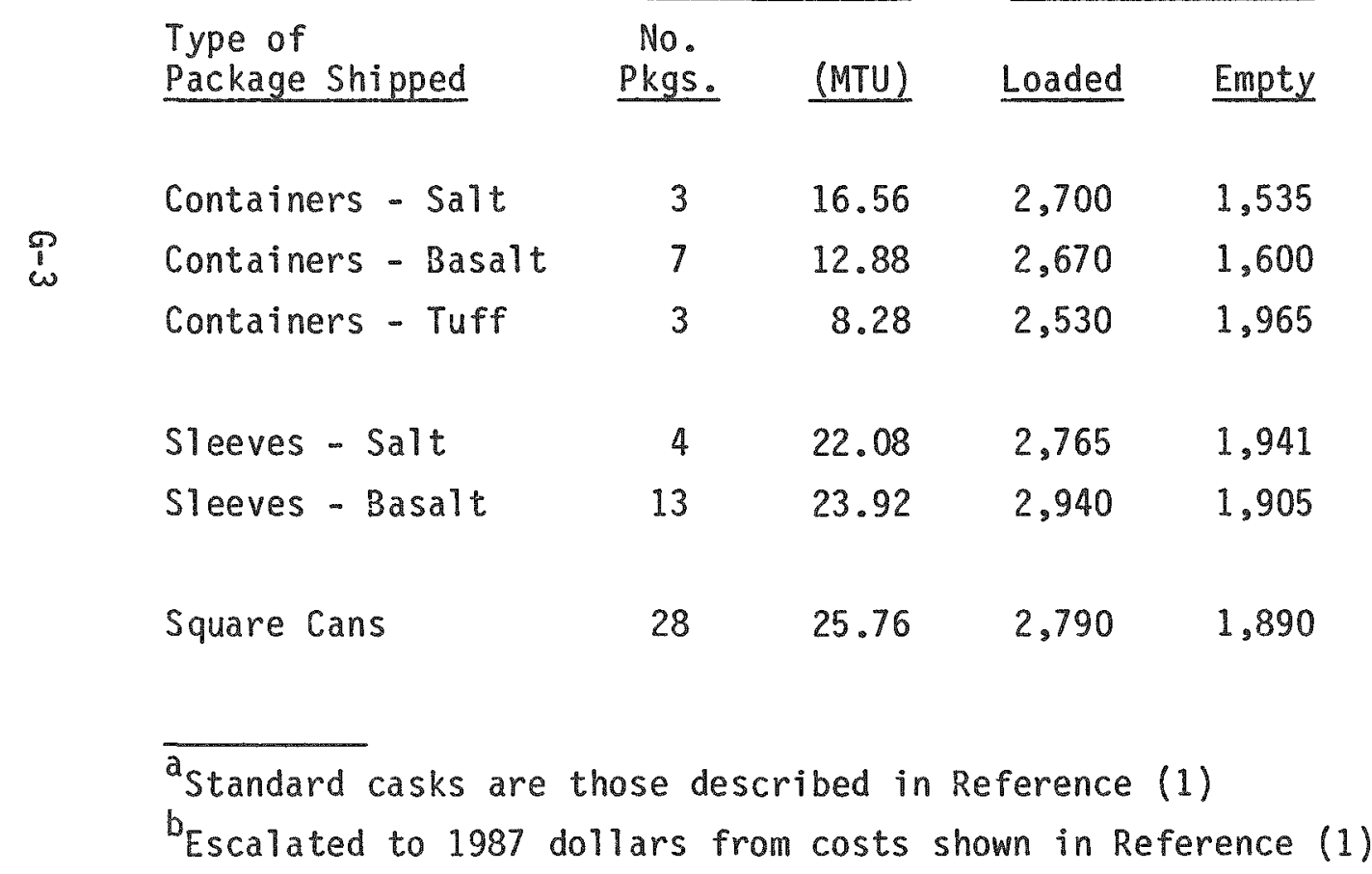

\begin{tabular}{|c|c|c|c|c|c|c|c|c|}
\hline \multicolumn{5}{|c|}{ "Standard" Transport Casks" } & \multicolumn{4}{|c|}{ TSCS } \\
\hline \multicolumn{2}{|c|}{ Capacity } & \multicolumn{2}{|c|}{ Weight (CWT) } & \multirow{2}{*}{$\begin{array}{l}\frac{\operatorname{cost}^{b}}{(\$ 000} \\
1987)^{2}\end{array}$} & \multicolumn{2}{|c|}{ Capacity } & \multicolumn{2}{|c|}{ Weight (CWT) } \\
\hline $\begin{array}{l}\text { No. } \\
\text { Pkgs. }\end{array}$ & (MTU) & Loaded & Empty & & $\begin{array}{r}\text { No. } \\
\text { Pkgs. }\end{array}$ & (MTU) & Loaded & Empty \\
\hline 3 & 16.56 & 2,700 & 1,535 & $\$ 2,867$ & 1 & 5.52 & 2,400 & 2,000 \\
\hline 7 & 12.88 & 2,670 & 1,600 & 2,980 & 4 & 7.36 & 2,610 & 2,000 \\
\hline 3 & 8.28 & 2,530 & 1,965 & 3,090 & 2 & 5.52 & 2,300 & 2,000 \\
\hline 4 & 22.08 & 2,765 & 1,941 & 3,199 & 3 & 16.56 & 2,600 & 2,000 \\
\hline 13 & 23.92 & 2,940 & 1,905 & 3,237 & 9 & 16.56 & 2,630 & 2,000 \\
\hline 28 & 25.76 & 2,790 & 1,890 & 3,169 & 21 & 19.31 & 2,300 & 2,000 \\
\hline
\end{tabular}




\subsection{ESTIMATED COSTS FOR TRANSPORT}

Separate calculations were made of the cost of shipping spent fuel from MRS facilities to the repository in the form of repository containers, sleeves, and square cans--using:
(1) the standard transport casks described in Reference (1)
(2) TSCs for one-way shipments
(3) TSCs for repetitive shipments.

The cask requirements for these shipments are shown in Table G-3 along with supporting data. 
TABLE G-3

CASK REQUIREMENTS FOR TRANSPORTING CONSOLIDATED FUEL FROM MRS TO REPOSITORY

FACILITIES IN CONTAINERS, SLEEVES \& CANS

\section{Description}

Fuel Content per Package (MTU)

No. Packages Annually a 3000 MTU/Yr

- Consolidated Rods

- Structural Parts

Cask Capacity (No. Packages)

- Standard Casks

- TSCs

No. Shipments Annually in

- Standard Casks

- TSCS

s

Shipping Distance One-Way (miles)

Speed (MPD)

Cask Availability (DPY)

No. Shipments/Year/Cask

No. Casks Required

- Standard Casks

- TSCS

\section{Containers for}

\begin{tabular}{|c|c|c|}
\hline Salt & Basalt & Tuff \\
\hline 5.52 & 1.84 & 2.76 \\
\hline $\begin{array}{l}543 \\
109\end{array}$ & $\begin{array}{r}1,631 \\
109\end{array}$ & $\begin{array}{r}1,087 \\
109\end{array}$ \\
\hline
\end{tabular}

$\begin{array}{lll}3 & 7 & 3 \\ 1 & 4 & 2\end{array}$

$\begin{array}{rrr}218 & 270 & 399 \\ 598 & 463 & 598 \\ 1,300 & 2,600 & 2,400 \\ 286 & 364 & 364 \\ 300 & 300 & 300 \\ 22.9 & 16.4 & 17.5\end{array}$

10
27

$17 \quad 23$

\begin{tabular}{|c|c|}
\hline Salt & Basalt \\
\hline 5.52 & 1.84 \\
\hline $\begin{array}{l}543 \\
109\end{array}$ & $\begin{array}{r}1,631 \\
109\end{array}$ \\
\hline
\end{tabular}

$\begin{array}{rr}4 & 13 \\ 3 & 9\end{array}$

$\begin{array}{rr}172 & 162 \\ 236 & 236 \\ 1,300 & 2,600 \\ 286 & 364 \\ 300 & 300 \\ 22.9 & 16.4\end{array}$

8

\begin{tabular}{|c|c|c|}
\hline \multicolumn{3}{|c|}{ Cans for } \\
\hline Salt & Basalt & Tuff \\
\hline 0.92 & 0.92 & 0.92 \\
\hline $\begin{array}{r}3,261 \\
109\end{array}$ & $\begin{array}{r}3,261 \\
109\end{array}$ & $\begin{array}{r}3,261 \\
109\end{array}$ \\
\hline $\begin{array}{l}28 \\
21\end{array}$ & $\begin{array}{l}28 \\
21\end{array}$ & $\begin{array}{l}28 \\
21\end{array}$ \\
\hline $\begin{array}{l}153 \\
210\end{array}$ & $\begin{array}{l}153 \\
210\end{array}$ & $\begin{array}{l}153 \\
210\end{array}$ \\
\hline 1,300 & 2,600 & 2,400 \\
\hline 286 & 364 & 364 \\
\hline 300 & 300 & 300 \\
\hline 22.9 & 16.4 & 17.5 \\
\hline
\end{tabular}

11

10
15

7

10

9

a Structural parts are packaged in tuff-type containers

b Number of TSCS required if used to replace entire fleet of standard casks. If TSC's were used for one-way shipment only and sufficient TSCS were available to replace fleet of standard casks, this number would represent sets of auxiliary equipment required for shipment. 
In addition the following basic assumptions were used in connection with the transport cost calculations:

(1) It was assumed that the casks would be shipped by special train with four casks being involved for each shipment.

(2) The special train charges amounted to $\$ 46.76 / \mathrm{mile}$, which represented the charges set forth in Reference (1), escalated to 1987 using the Rail Freight Index.

(3) The annual cask operating costs associated with the shipment were the same as those described in Section 2.3 of Appendix 0.

(4) The freight charges associated with the shipments were determined using the algorithms developed in Section 2.1 of Appendix D.

(5) The security charges associated with the shipment were determined using the methodology described in Section 2.2 of Appendix D.

(6) Life cycle costs were determined over the 25 year period 1998-2022 and discounted at 3 percent/year to 1987. Unit costs were determined as described in Section 3.0 of Appendix D.

The following Table G-4 sets forth the unit costs for transporting spent fuel in the various packages from MRS to repository facilities in both standard transport casks and TSCS. 
TABLE G-4

ESTIMATED COST OF TRANSPORTING SPENT FUEL FROM

MRS TO REPOSITORY FACILITIES

Cost of Transport (\$/kgU)

$\begin{array}{lrrr}\text { MRS Facilities } & \begin{array}{c}\text { Standard } \\ \text { Transport } \\ \text { Casks }\end{array} & \begin{array}{c}\text { Tne-Way } \\ \text { Use }\end{array} & \begin{array}{c}\text { Repetitive } \\ \text { Use }\end{array} \\ \text { To Salt Repository } & \$ 5.73 & & \$ 9.58 \\ \text { - in Containers } & 4.63 & 4.06 & \$ 14.02 \\ \text { - in Sleeves } & 4.12 & 3.66 & 5.72 \\ \text { - in Cans } & & & \\ \text { To Basalt Repository } & 11.18 & 13.05 & 17.87 \\ \text { - in Containers } & 7.01 & 6.68 & 9.14 \\ \text { - in Sleeves } & 6.53 & 5.98 & 8.16 \\ \text { - in Cans } & & & \\ \text { To Tuff Repository } & 15.37 & 15.54 & 21.47 \\ \text { - in Containers } & 6.12 & 5.63 & 7.71 \\ \text { - in Cans } & & & \end{array}$

From the results shown in the table, it can be concluded that standard transport casks are less expensive to use for transporting packages of consolidated fuel from MRS to repository facilities than are TSCS when the latter are used to replace standard transport casks in repetitive shipments. Even if higher payload capacities (20-25\%) were feasible for TSCs, there would be no savings involved over the use of a standard transport cask specifically designed for the payload to be shipped.

The one-way use of TSCs, if such should be available in sufficient quantities, can result in savings in the cost of transport over that involved with the use of standard casks in cases where the spent fuel is contained in sleeves or cans. Moreover, if higher payload capacities (20-25 percent) were feasible, savings would result in all cases with the exception of shipments involving spent fuel in salt repository containers. However, in order to replace a single standard cask it would be necessary to receive 23 TSCs/year for shipments to a salt repository, 17 TSCS/year for shipments to a basalt repository, or $18 \mathrm{TSCS} /$ year for shipments to a tuff repository--for a period 
of 25 consecutive years (totals of 575 TSCs for salt, 425 TSCs for basalt, and 450 TSCs for tuff). Under such circumstances the magnitude of the savings involved in the use of TSCS would range from $\$ 0.33-\$ 0.57 / \mathrm{kgU}$ using the payloads assumed for this study, and from $\$ 0.74-\$ 2.94 / \mathrm{kgU}$ using payloads which were 20 percent higher.

For any of these savings to be realized, it would be necessary that utilities commit to provide TSCS early enough, and to deliver the TSCS on a schedule compatible with DOE needs, such that DOE does not have to otherwise commit to provide all or a portion of a fleet of rail casks for its use.

\subsection{REFERENCES FOR APPENDIX G}

(1) E. R. Johnson Associates, Inc., Assessment of the Use of a Multi-Purpose and Centralized Facility for the Disassembly and Packaging of Spent Nuclear Fuel to Support the Various Segments of the DOE Waste Management system, JAI-254, DOE Contract No. DE-ACO1-84RW00037 
APPENDIX H

ESTIMATED COSTS FOR SALVAGE OR DISPOSAL OF USED TSCS, SOCS

AND CONCRETE STORAGE CASKS 
APPENDIX H

ESTIMATED COSTS FOR SALVAGE OR DISPOSAL OF USED TSCS, SOCS

AND CONCRETE STORAGE CASKS

\section{TABLE OF CONTENTS}

$\begin{array}{lll}1.0 & \text { METAL CASKS } & \frac{\text { Page }}{2} \\ 2.0 & \text { CONCRETE STORAGE CASKS } & \mathrm{H}-1 \\ 3.0 & \text { CONCLUSIONS REGARDING MODULE DISPOSAL COSTS } & \mathrm{H}-2 \\ & \mathrm{H}-3\end{array}$




\section{APPENDIX H}

ESTIMATED COSTS FOR SALVAGE OR DISPOSAL OF USED TSCS, SOCS

AND CONCRETE STORAGE CASKS

The purpose of this Appendix is to develop estimates of costs for salvage or disposal of storage modules at the end of their useful life. While these estimates have been based on decontamination, disassembly, etc. at the reactor, essentially identical costs are to be expected if such work were performed at DOE facilities.

\section{$1.0 \quad$ METAL CASKS}

A preliminary estimate was made of the cost of both salvage and disposal of metal storage casks (TSCS and SOCs). However, it should be pointed out here that there are uncertainties about the acceptability of nuclear equipment as salvage, even when thoroughily decontaminated.

In the case of prospective salvage, the cost of a thorough decontamination was estimated to require about 20 person-days, and to cost $\$ 10,080$. The cost of shipping the fully decontaminated cask to the salvage site 200 miles away was estimated to be $\$ 7,740$, assuming that a Class $37 \frac{1}{2}$ freight rate would apply. The value of the cask as scrap steel was estimated at $\$ 25 /$ ton, for a total of about $\$ 2,500$. Other metals (such as lead) would be appreciably more. Offsetting this prospectively higher value is the fact that the cask would either have to be delivered as a nominal 100-ton object or subject to expensive size reduction operation. In addition, the cost of handling the cask at the reactor site, after unloading, to the point of shipment to the salvage site was estimated to be about $\$ 740$ (11.75 person-hours). The net result of the foregoing is that if the salvage route is taken to dispose of the cask, the net cost is about $\$ 16,060 /$ cask (1987 dollars). If the decontamination turns out to be minimal, this cost could drop to as low as $\$ 9,000 /$ cask.

In the case of disposal of a cask without salvaging any of the materials of construction, the cost of handling the cask (including removal of loose contamination on the surface of the cask) was estimated to be $\$ 1,764$ (28 
person-hours). The cost of shipping the cask to a disposal site 900 miles away was estimated to be $\$ 18,495$ assuming that a class $37 \frac{1}{2}$ freight rate would apply. Assuming the cask (volume- -520 cubic feet) would be buried in a low level waste burial ground on an as-received basis, the cost was estimated to be $\$ 35,084$, broken down as follows:

$\begin{array}{lr}\text { Base Disposal Charge } & \$ 16,380 \\ \text { Surcharge (Weight) } & 13,108 \\ \text { Cask Handl ing Fee } & 800 \\ \text { Funds } & 3,973 \\ \text { Tax (2.4\% of above) } & 823 \\ \quad \text { Total } & \$ 35,084\end{array}$

The net result of the foregoing is that the cost of disposal of the cask is about $\$ 55,343$. A summary of these costs is set forth in Table $\mathrm{H}-1$.

TABLE H-1

ESTIMATED COSTS FOR DISPOSAL/SALVAGE OF METAL STORAGE CASKS

(1987 Dollars)

Description

Cask Handling at Reactor

Decontamination

Transport of Cask to Disposal or Salvage Site

\begin{tabular}{|c|c|c|}
\hline \multirow{3}{*}{ tor } & \multicolumn{2}{|c|}{ Cost } \\
\hline & Salvage & Disposa \\
\hline & $\$ 740$ & $\$ 1,764$ \\
\hline & 10,080 & - \\
\hline Disposal or Salvage Site & 7,740 & 18,495 \\
\hline & - & 35,084 \\
\hline & $(2,500)$ & $=$ \\
\hline & $\$ 16,060$ & $\$ 55,343$ \\
\hline
\end{tabular}

Disposal at Barnwe11

Salvage value

Total

\subsection{CONCRETE STORAGE CASKS}

A preliminary estimate was also made of the cost of disposal of concrete storage casks. It was assumed that the inner metal containment vessel of the cask and associated plugs would be removed and packaged for disposal at a low level waste burial site. The remainder of the concrete monolith would be broken up and disposed of in a land fill. The following Table H-2 summarizes the estimated costs for disposal of concrete storage casks. 
TABLE H-2

ESTIMATED COSTS FOR DISPOSAL OF CONCRETE STORAGE CASKS

(1987 Dollars)

\begin{tabular}{lr} 
Description & Cost \\
\hline Cask Handing at Reactor & $\$ 462$ \\
Removal of Inner Vessel & 3,024 \\
Transport of Vessel to Barnwell & 2,300 \\
Disposal of Vessel at Barnwell & 4,726 \\
Breakup of Concrete Shell & 6,640 \\
Load and Transport to Land Fill & 1,250 \\
Disposal at Land Fill & 1,000 \\
\hline Total & $\$ 19,402$
\end{tabular}

Since the storage capacity of a concrete cask is 4.291 MTU (vs 9.26 MTU for a metal cask), the foregoing cost is essentially equivalent to about a $\$ 42,000$ disposal cost for a metal storage cask.

\subsection{CONCLUSIONS REGARDING MODULE DISPOSAL COSTS}

Based on the preliminary cost estimates developed in the preceding sections it is reasonable to conclude that concrete casks may be less expensive to dispose of at the end of their useful 1ife than metal casks, if the metal casks indeed need to be disposed of. However, if metal casks should prove to be salvageable, considerably lower disposal costs would result. Moreover, the equipment used to transfer spent fuel to and from the concrete casks will have to be decontaminated and disposed of (or salvaged) at the end of its useful life, and the cost of this activity would have to be accrued over the number of concrete casks serviced. Thus the cost of disposition of a metal cask can be expected to range between $\$ 26,000$ less expensive $(\$ 2.81 / \mathrm{kgU}$ for casks storing intact fuel assemblies and $1.69 / \mathrm{kgl}$ for casks storing consolidated fuel), to being $\$ 13,000$ more expensive $(\$ 1.40 / \mathrm{kgU}$ for casks storing intact fuel assemblies and $\$ 0.85 / \mathrm{kg}$ for casks storing consolidated fuel) than concrete storage casks of equivalent capacity.

Some savings in disposal costs of both cask types might be realized by loading the metal casks or the inner vessel of the concrete casks with other low level wastes. 
APPENDIX I

SENSITIVITY OF COSTS TO VARIATIONS IN THE CAPACITY AND FABRICATION COSTS OF METAL STORAGE CASKS 
APPENDIX I

SENSITIVITY OF COSTS TO VARIATIONS IN THE CAPACITY

AND FABRICATION COSTS OF METAL STORAGE CASKS

\section{TABLE OF CONTENTS}

Page

1.0 SENSITIVITY TO CHANGES IN THE TSC AND SOC CAPACITY I-I

1.1 Sensitivity of At-Reactor Costs to Changes in the I-2

Capacity of Metal Storage Casks

1.1.1 Sensitivity of At-Reactor Operations Costs to I-2

Changes in Cask Capacity

1.1.2 Sensitivity of At-Reactor Life Cycle and Unit I-7

Costs to Changes in Cask Capacity

1.1.3 Discussion of Cost Comparisons

1.1.3.1 Comparison of Costs for At-Reactor Storage as Intact Fuel Assemblies

1.1.3.2 Comparison of Costs for At-Reactor Storage as Consolidated Fuel

I -27

$I-27$

$[-27$

1.2 Sensitivity of Savings Within the DOE System to Changes I-28
in the Capacity of Metal Storage Casks

1.2.1 Sensitivity of Savings in the cost of Transport I-28 from Reactors to DOE Facilities to Changes in Cask Capacity

1.2.2 Sensitivity of Savings from Replacement of I-30 Lag Storage Capacity to Changes in Cask Capacity

1.2.3 Sensitivity of Savings from Replacement of I-32 MRS Modules to Changes in Cask Capacity

1.2.4 Sensitivity of the Cost of Transport from the I-34 MRS to a Repository to Changes in Cask Capacity

2.0 SENSITIVITY TO CHANGES IN THE COST OF TSCS OR SOCS I-36

2.1 Sensitivity of At-Reactor Costs to Changes in the Cost I-36

for Metal Storage Casks

2.1.1 Sensitivity of At-Reactor Operations costs to I-36

Changes in Cask Cost

2.1.2 Sensitivity of At-Reactor Life Cycle and Unit I-36 Costs to Changes in Cask cost

2.2 Sensitivity of Savings Within the DOE System to Changes I-38

in the Cost of Metal Storage Casks

2.2.1 Sensitivity of Savings in the Cost of Transport I-38 from Reactors to DOE Facilities to Changes in Cask Cost 


\section{APPENDIX I}

TABLE OF CONTENTS (Continued)

Page

2.2.2 Sensitivity of Savings from Replacement of Lag Storage Capacity to Changes in Cask Cost

2.2.3 Sensitivity of Savings from Replacement of MRS Modules to Changes in Cask cost

2.2.4 Sensitivity of the cost of Transport from the MRS to a Repository to Changes in Cask Cost

3.1 Sensitivity of Costs At Reactor Sites 


\section{APPENDIX I}

\section{SENSITIVITY OF COSTS TO VARIATIONS IN THE CAPACITY}

AND FABRICATION COSTS OF METAL STORAGE CASKS

The purpose of this Appendix is to determine the sensitivity of costs/savings associated with the use of metal storage casks in the utility and DOE spent fuel management systems to variations in the capacity of the casks and the cost of fabrication thereof. Conclusions are developed regarding the relative sensitivity of each of the components of cost considered in this study (i.e., at-reactor costs, transport costs, and costs/savings at DOE facilities) to these variations.

1.0 SENSITIVITY TO CHANGES IN THE TSC AND SOC CAPACITY

The TSCS and SOCs considered in the study were assumed to have a capacity for storing 21 PWR fuel assemblies or 46 BWR fuel assemblies (average of 9.26 MTU for intact fuel assemblies and 15.37 MTU for consolidated fuel). This cask capacity will be referred to as the base case in this sensitivity analysis. Two larger capacity casks were evaluated:

$$
\begin{aligned}
\text { Case A - A TSC or SOC capacity of } 26 \text { PWR assemblies or } 52 \text { BWR } \\
\text { assemblies (average of } 11.16 \text { MTU for intact fuel } \\
\text { assemblies and } 18.53 \text { MTU for consolidated fuel) } \\
\text { Case B - A TSC or SOC capacity of } 32 \text { PWR assemblies or } 76 \text { BWR } \\
\text { assemblies (average of } 14.47 \text { MTU for intact fuel } \\
\text { assemblies and } 24.02 \text { MTU for consolidated fuel) }
\end{aligned}
$$

These cases were selected as being representative of future metal cask designs that could allow for more densely packed spent fuel assemblies. Case B is considered to be the upper bound of capacity for these metal casks.

In Appendix $C$ it was assumed that there were fundamental similarities between the designs of the transport cask and the TSC or SOC. These metal casks were all assumed to have the same capacity (i.e., 21 PWR or 46 BWR assemblies). For the purpose of the sensitivity analysis it is assumed that if higher capacity TSCS and SOCs would be available for use in the waste management systems, correspondingly higher capacity transport casks would also be available. 
Since this portion of the sensitivity analysis only considered impacts of changes in cask capacity, the cost of the casks was assumed to be the same as that used in Appendix $C$.

1.1 SENSITIVITY OF AT-REACTOR COSTS TO CHANGES IN THE CAPACITY OF METAL STORAGE CASKS

1.1.1 Sensitivity of At-Reactor Operations Costs to Changes in Cask Capacity

An analysis was performed of the impact of increased capacity metal casks on the costs of operation at a reactor site. For this analysis, it was assumed that the increased capacity casks were not dimensionally larger or appreciably heavier than the base case cask. Therefore, appreciably higher capacity handling equipment at the reactor would not be needed.

The at-reactor operations costs for receiving, loading and transfer to storage of increased capacity TSCS and SOCS were estimated. Operations costs for removal from storage, unloading, fuel inspection, and receiving and loading transport casks or overpacks for the increased capacity SOC options were also estimated.

Only activities involving the handling of individual spent fuel assemblies increased in cost for higher capacity metal casks. The cost per cask of these activities increased proportionately with the increased number of assemblies in the cask. However, it is important to point out that the unit cost $(\$ / \mathrm{kgU})$ for these activities remained unchanged from the base case. The total unit costs for at-reactor operations decrease with increasing metal storage cask capacity. This is because the large majority of the operations involve handling the cask as a whole and the costs of these operations do not increase with increased cask capacity. The unit costs for at-reactor operations costs for each of the higher capacity metal cask options for intact and consolidated fuel are set forth in Tables I-1 through I-4. The percent decrease in at-reactor operations costs for handling TSCS or SOCs is somewhat less than the percent increase in cask capacity. However, it should be noted that the at-reactor operations costs are only a small portion of the total atreactor costs. The cost of the casks is the large portion of the total atreactor costs as will be seen in the next section. Therefore, even a large decrease in the at-reactor operations costs will not significantly change the total at-reactor costs. 
TABLE I-1

SUMMARY OF ESTIMATED AT-REACTOR HANDLING COSTS ASSOCIATED WITH DRY STORAGE MODULES

(Increased Capacity Metal Casks -- Case A (26/52) Intact Fuel Assemblies)

(1) Loading \& Placement in AR Storage
(a) Cask Receiving \& Placement in Pool
(b) Cans
(c) Canning
(d) Loading Transfer to Storage
(e) Equi pment Rental
Subtotal

\begin{tabular}{|c|c|c|c|c|}
\hline \multirow[b]{2}{*}{$\begin{array}{l}\text { Section } \\
\text { Reference }\end{array}$} & \multicolumn{4}{|c|}{ Average Cost $(\$ / \mathrm{kgu}, 1987)^{2}$} \\
\hline & $\begin{array}{c}\text { TSC or SOC } \\
\text { Destined For } \\
\text { Shipment } \\
\text { To DOE }\end{array}$ & $\begin{array}{c}\text { SOC } \\
\text { Shipped To } \\
\text { DoE In } \\
\text { Overpack } \\
\end{array}$ & $\begin{array}{c}\text { SOC } \\
\text { Used For } \\
\text { At-Reactor } \\
\text { Storage only } \\
\end{array}$ & $\begin{array}{c}\text { Concrete } \\
\text { Cask } \\
\end{array}$ \\
\hline $\begin{array}{l}1.0 \\
= \\
3.1 \\
2.0,3.2,3.3 \\
\text { App A, 3.0 }\end{array}$ & $\begin{array}{r}\$ 0.19 \\
- \\
0.42 \\
- \\
\end{array}$ & $\begin{array}{r}\$ 0.19 \\
- \\
0.42 \\
\end{array}$ & $\begin{array}{r}\$ 0.19 \\
- \\
0.42 \\
\end{array}$ & $\begin{array}{l}\$ .03 \\
9.52 \\
3.49 \\
1.45 \\
\end{array}$ \\
\hline & 0.61 & 0.61 & 0.61 & 14.49 \\
\hline
\end{tabular}

(2) Removal From AR Storage Shipment Preparation

(a) Removal from Storage \& Unloading

(b) Decanning

(c) Can Disposal

(d) Fuel Inspection

(e) Receiving of Transport Cask

(f) Loading of Transport Cask

(g) Equi pment Rental

(h) Preparation of TSC or SOC for Shi pment

(i) Overpacking of SOC for shipment Subtotal

$4.0,6.0$
6.0
6.0
7.0
1.0
8.0
6.0
5.0
9.0

Total

\begin{tabular}{rrrr}
- & - & 0.26 & 3.25 \\
- & - & - & 0.52 \\
- & - & 1.60 & 1.15 \\
- & - & 0.19 & 0.22 \\
$\vdots$ & - & 0.45 & 0.48 \\
0.10 & - & - & 1.45 \\
\hline 0.10 & 0.62 & -1.66 & - \\
$\$ 0.71$ & 0.62 & 2.50 & 8.73 \\
\hline & $\$ 1.23$ & $\$ 3.11$ & $\$ 23.22$
\end{tabular}

a Based on: $2 / 3$ of the amount ( $\mathrm{kgU}$ ) of fuel being PWR fuel, with an average of $461 \mathrm{kgU} / \mathrm{assembly}$, and a cask capacity of 26 assemblies; $1 / 3$ of the amount ( $\mathrm{kgU}$ ) of fuel being BWR fuel, with an average of $183 \mathrm{kgU} / \mathrm{assembly}$, and a cask capacity of 52 assemblies. Concrete casks assumed to have a capacity for storing 9 PWR or 25 BWR assemblies.

$b$

References are to Sections in Appendix B unless otherwise indicated. (Note: Costs developed in these sections were adjusted for increased capacity metal casks.)

C Included in the costs shown in $(1)(d)$ 
(Increased Capacity Metal Casks - - Case B (32/76) Intact Fuel Assemblies)

(1) Loading \& Placement in AR Storage
(a) Cask Receiving \& Placement in Pool
(b) Cans
(c) Canning
(d) Loading \& Transfer to Storage
(e) Equipment Rental
Subtotal

(2) Removal From AR Storage Shipment Preparation

(a) Removal from Storage \& Unloading

(b) Decanning

(c) Can Disposal

(d) Fuel Inspection

(e) Receiving of Transport Cask

(f) Loading of Transport Cask

(g) Equipment Rental

(h) Preparation of TSC or SOC for Shipment

(i) Overpacking of SOC for shipment Subtotal

\begin{tabular}{|c|c|c|c|c|}
\hline \multirow[b]{2}{*}{$\begin{array}{l}\text { Section } \\
\text { Reference }\end{array}$} & \multicolumn{4}{|c|}{ Average Cost $(\$ / \mathrm{kgu}, 1987)^{\mathrm{a}}$} \\
\hline & $\begin{array}{l}\text { TSC or SOC } \\
\text { Destined For } \\
\text { Shipment } \\
\text { To DOE }\end{array}$ & $\begin{array}{c}\text { SOC } \\
\text { Shipped To } \\
\text { DOE In } \\
\text { Overpack }\end{array}$ & $\begin{array}{c}\text { SOC } \\
\text { Used For } \\
\text { At-Reactor } \\
\text { Storage only }\end{array}$ & $\begin{array}{c}\text { Concrete } \\
\text { Cask }\end{array}$ \\
\hline
\end{tabular}

$\begin{array}{r}\$ 0.14 \\ -\overline{-} \\ 0.38 \\ \hline 0.52\end{array}$

$\begin{array}{r}\$ 0.14 \\ - \\ 0.38 \\ \hline 0.52\end{array}$

$\begin{array}{r}\$ 0.14 \\ \overline{-} \\ 0.38 \\ \hline 0.52\end{array}$

\begin{tabular}{l}
$\$ .03^{-c}$ \\
0.52 \\
3.49 \\
1.45 \\
\hline 14.49
\end{tabular}

Total

$4.0,6.0$
6.0
6.0
7.0
1.0
8.0
6.0
5.0
9.0

1.0
-
3.1
$2.0,3.2$,
App A, 3.


$4.0,6.0$
6.0
6.0
7.0
1.0
8.0
6.0
5.0
9.0

Based on: $2 / 3$ of the amount ( $\mathrm{kgU}$ ) of fuel being PWR fuel, with an average of $461 \mathrm{kgU} / \mathrm{assembly}$, and a cask capacity of 32 assemblies; $1 / 3$ of the amount ( $\mathrm{kgU}$ ) of fuel being BWR fuel, with an average of $183 \mathrm{kgU} / \mathrm{assembly}$, and a cask capacity of 76 assemblies. Concrete casks assumed to have a capacity for storing 9 PWR or 25 BWR assemblies. References are to Sections in Appendix B unless otherwise indicated. (Note: Costs developed in these sections were adjusted for increased capacity metal casks.)

C Included in the costs shown in (1)(d) 
TABLE I-3

SUMMARY OF ESTIMATED AT-REACTOR HANDLING COSTS ASSOCIATED WITH DRY STORAGE MODULES

(Increased Capacity Metal Casks - Case A (26/52) Consolidated Fuel)

(1) Loading \& Placement in AR Storage

(a) Cask Receiving \& Placement in Pool

(b) Cans

(c) Canning

(d) Loading \& Transfer to Storage

(e) Equipment Rental

Subtotal

\begin{tabular}{|c|c|c|c|c|}
\hline \multirow[b]{2}{*}{$\begin{array}{l}\text { Section } \\
\text { Reference }\end{array}$} & \multicolumn{4}{|c|}{ Average Cost $(\$ / \mathrm{kgU}, 1987)^{\mathrm{a}}$} \\
\hline & $\begin{array}{l}\text { TSC or SOC } \\
\text { Destined For } \\
\text { Shipment } \\
\text { To DOE }\end{array}$ & $\begin{array}{c}\text { SOC } \\
\text { Shipped To } \\
\text { DOE In } \\
\text { Overpack }\end{array}$ & $\begin{array}{c}\text { SOC } \\
\text { Used For } \\
\text { At-Reactor } \\
\text { Storage Only }\end{array}$ & $\begin{array}{c}\text { Concrete } \\
\text { Cask }\end{array}$ \\
\hline
\end{tabular}

\begin{tabular}{|c|c|c|c|c|}
\hline 1.0 & $\$ 0.11$ & $\$ 0.11$ & $\$ 0.11$ & - \\
\hline 3.1 & - & - & - & $-c$ \\
\hline $2.0,3.2,3.3$ & 0.25 & 0.25 & 0.25 & 1.99 \\
\hline & 0.36 & 0.36 & 0.36 & 3.25 \\
\hline
\end{tabular}

(2) Removal From AR Storage \& Shipment Preparation

(a) Removal from Storage \& Unloading

(b) Decanning

(c) Can Disposal

(d) Fuel Inspection

(e) Receiving of Transport Cask

(f) Loading of Transport Cask

(g) Equipment Rental

(h) Preparation of TSC or SOC for Shipment

(i) Overpacking of SOC for shipment Subtotal

4.0 .6 .0
6.0
6.0
7.0
1.0
8.0
6.0
5.0
9.0

Total

.0 $\overline{0.06}$

$\$ 0.42$

$=$
$=$
$=$
$=$
0.06
0.06
$\$ 0.42$

0.16<smiles>[Li][13IH]</smiles>

0.48

0.11

0.27$$
\begin{array}{r}
0.27 \\
-
\end{array}
$$$$
\frac{0.37}{0.37}
$$$$
\frac{-}{1.02}
$$

$\$ 0.73$

$\$ 1.38$
1.83

0.50

0.13

0.29

1.26

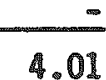

$\$ 7.26$

\footnotetext{
Based on: 2/3 of the amount (kgU) of fuel being PWR fuel, with an average of $770 \mathrm{kgU} / \mathrm{can}$, and a cask capacity of 26 cans: $1 / 3$ of the amount (kgU) of fuel being BWR fuel, with an average of $306 \mathrm{kgu} / \mathrm{can}$, and a cask capacity of 52 cans. Concrete casks assumed to have a capacity for storing 9 PWR cans or 25 BWR cans.

References are to Sections in Appendix B unless otherwise indicated. (Note: Costs developed in these sections were adjusted for increased capacity metal casks.)

c Included in the costs shown in (1)(d)

Consolidated fuel assumed to be canned already
} 
(Increased Capacity Metal Casks - Case B (32/76) Consolidated Fuel)

\begin{tabular}{ccccc} 
& \multicolumn{4}{c}{ Average Cost $(\$ / \mathrm{kgU}, 1987)^{\mathrm{a}}$} \\
\hline & TSC or SOC & SOC & SOC \\
Destined For & Shipped To & Used For & \\
Section & Shipment & DOE In & At-Reactor & Concrete \\
Reference & To DOE & Overpack & Storage Only & Cask
\end{tabular}

(1) Loading \& Placement in AR Storage
(a) Cask Receiving \& Placement in Pool
(c) Canning
(d) Loading \& Transfer to Storage
(e) Equi pment Rental
Subtotal

$\begin{array}{lr}1.0 & \$ 0.08 \\ = & - \\ 3.1 & - \\ 2.0,3.2 .3 .3 & 0.23 \\ \text { App A.3.0 } & \frac{0.31}{}\end{array}$

$\begin{array}{r}\$ 0.08 \\ - \\ 0.23 \\ \hline 0.31\end{array}$

$\$ 0.08$

(2) Removal From AR Storage \& Shipment

$\begin{array}{ll}\text { (a) Removal from Storage \& Unloading } & 4.0 .6 .0 \\ \text { (b) Decanning } & 6.0 \\ \text { (c) Can Disposal } & 6.0 \\ \text { (d) Fuel Inspection } & 7.0 \\ \text { (e) Receiving of Transport Cask } & 1.0 \\ \text { (f) Loading of Transport Cask } & 8.0 \\ \text { (g) Equipment Rental } & 6.0 \\ \text { (h) Preparation of TSC or SOC for } & 5.0 \\ \text { (i) Ovipinent } & \\ \text { Subtotal } & 9.0\end{array}$

Total

\begin{tabular}{rrrr}
- & - & 0.13 & 1.83 \\
- & - & - & - \\
- & - & 0.49 & 0.50 \\
- & - & 0.08 & 0.13 \\
- & - & 0.24 & 0.29 \\
0.05 & - & - & 1.26 \\
\hline 0.05 & 0.32 & -1.94 & - \\
$\$ 0.36$ & $\$ 0.63$ & $\$ 1.25$ & $\$ 7.26$
\end{tabular}

\footnotetext{
Based on: 2/3 of the amount (kgU) of fuel being PWR fuel, with an average of $770 \mathrm{kgU} / \mathrm{can}$, and a cask capacity of 32 cans; $1 / 3$ of the amount ( $\mathrm{kgU}$ ) of fuel being BWR fuel, with an average of $306 \mathrm{kgU} / \mathrm{can}$, and a cask capacity of 76 cans. Concrete casks assumed to have a capacity for storing 9 PWR cans or 25 BWR cans.

b References are to Sections in Appendix B unless otherwise indicated. (Note: Costs developed in these sections were adjusted for increased capacity metal casks.)

c Included in the costs shown in (1)(d)

d Consolidated ruel assumed to be canned already
} 
1.1.2 Sensitivity of At-Reactor Life Cycle and Unit Costs to Changes in Cask Capacity

An analysis was performed of the impact of increased capacity metal casks on the life cycle and resulting unit costs involved with the use of the casks at the reactor site. The analysis was performed using the same methodology as was used for determining the at-reactor cask costs for the base case (as described in Appendix C). The number of additional metal storage casks needed each year was calculated by dividing the amount of fuel requiring storage each year by the cask capacity. The resulting number of metal storage casks was multiplied by the estimated cost of a cask to determine the total cask cost to the utility system for a given year. The calculations were made for the total number of years that additional at-reactor storage is projected to be required; this is dependent on when the DOE system begins receiving spent fuel. The following cases were evaluated for the on-time repository (1998) scenario:

\begin{tabular}{|c|c|c|c|c|}
\hline $\begin{array}{l}\text { Case } \\
\text { No. }\end{array}$ & $\begin{array}{l}\text { Type of } \\
\text { Cask Used } \\
\text { For AR } \\
\text { Storage } \\
\end{array}$ & $\begin{array}{c}\text { Cask Capacity } \\
\text { Number } \\
\text { of Ass embiles } \\
\text { (PUR/BWR) }\end{array}$ & $\begin{array}{l}\text { Type of } \\
\text { Fuel }\end{array}$ & $\begin{array}{l}\text { Method of } \\
\text { Shipment to DOE Facilities }\end{array}$ \\
\hline $\begin{array}{l}\text { A1 } \\
\text { A2 } \\
\text { A3 } \\
\text { A4 } \\
\text { A5 }\end{array}$ & $\begin{array}{l}\text { TSC } \\
\text { TSC } \\
\text { SOC } \\
\text { SOC } \\
\text { SOC }\end{array}$ & $\begin{array}{l}(26 / 52) \\
(26 / 52) \\
(26 / 52) \\
(26 / 52) \\
(26 / 52)\end{array}$ & $\begin{array}{l}\text { Intact assemblies } \\
\text { Consolidated fuel } \\
\text { Intact assembiies } \\
\text { Consolidated fuel } \\
\text { Intact assemblies }\end{array}$ & $\begin{array}{l}\text { In TSC } \\
\text { In TSC } \\
\text { In DOE-furnished transport cask } \\
\text { In DOE-furnished transport cask } \\
\text { In SOC; one-time use of SOC for } \\
\text { shipment }\end{array}$ \\
\hline A6 & SOC & $(26 / 52)$ & Consolidated fuel & $\begin{array}{l}\text { In soc; one-time use of SOC for } \\
\text { shipment }\end{array}$ \\
\hline $\begin{array}{l}\text { A7 } \\
\text { A8 }\end{array}$ & $\begin{array}{l}\text { SOC } \\
\text { SOC }\end{array}$ & $\begin{array}{l}(26 / 52) \\
(26 / 52)\end{array}$ & $\begin{array}{l}\text { Intact assemblies } \\
\text { Consolidated fuel }\end{array}$ & $\begin{array}{l}\text { In SOC in overpack } \\
\text { In SOC in overpack }\end{array}$ \\
\hline $\begin{array}{l}B 1 \\
B 2 \\
B 3 \\
B 4 \\
B 5\end{array}$ & $\begin{array}{l}\text { TSC } \\
\text { TSC } \\
\text { SOC } \\
\text { SOC } \\
\text { SOC }\end{array}$ & $\begin{array}{l}(32 / 76) \\
(32 / 76) \\
(32 / 76) \\
(32 / 76) \\
(32 / 76)\end{array}$ & $\begin{array}{l}\text { Intact assemblies } \\
\text { Consolidated fue } \\
\text { Intact assemblies } \\
\text { Consolidated fuel } \\
\text { Intact assemblies }\end{array}$ & $\begin{array}{l}\text { In TSC } \\
\text { In TSC } \\
\text { In DOE-furnished transport cask } \\
\text { In DOE-furnished transport cask } \\
\text { In SOC; one-time use of SOC for } \\
\text { shipment }\end{array}$ \\
\hline$B 6$ & SOC & $(32 / 76)$ & Consolidated fuel & $\begin{array}{l}\text { In soc; one-time use of soc for } \\
\text { shipment }\end{array}$ \\
\hline $\begin{array}{l}B 7 \\
B 8\end{array}$ & $\begin{array}{l}\text { SOC } \\
\text { SOC }\end{array}$ & $\begin{array}{l}(32 / 76) \\
(32 / 76)\end{array}$ & $\begin{array}{l}\text { Intact assemblies } \\
\text { Consolidated fuel }\end{array}$ & $\begin{array}{l}\text { In SOC in overpack } \\
\text { In SOC in overpack }\end{array}$ \\
\hline
\end{tabular}

The impact of increased capacity metal casks on the total cost of metal casks at the reactor is significant. Fewer casks are needed to meet the storage requirements, thereby reducing the total cost of casks in the utility system. The results of the cost calculations for the TSC and SOC higher capacity cases 
are included in Tables I-5 through I-20. These tables list the at-reactor costs for spent fuel stored as intact fuel assemblies and in consolidated form in cans. The at-reactor operations costs are also included in the tables and added to the cask costs to provide total at-reactor costs. The unit costs are calculated (using a $3 \%$ discount rate) and also provided in the tables. Summaries of the at-reactor costs for Cases A and B are provided in Tables I21 and I-22. The summary tables provide a comparison of the at-reactor costs (in $\$ / \mathrm{kg} U$ ) for the base case, each of the increased capacity metal cask options, and the concrete cask option. 
IALLE I-S

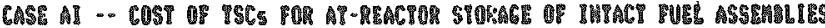

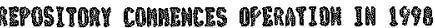

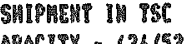

(50\% of ar Storage Requsreaents Provided by basks)

\begin{tabular}{|c|c|c|c|c|c|c|c|c|c|c|}
\hline \multirow{6}{*}{ YRAR } & \multirow{6}{*}{\multicolumn{2}{|c|}{ 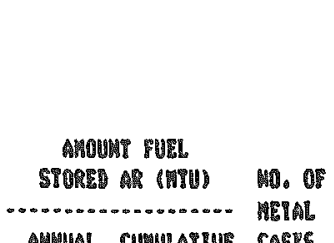 }} & \multirow{6}{*}{ 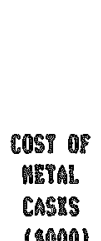 } & \multirow{6}{*}{$\begin{array}{l}\text { COST OP } \\
\text { LOAQIME } \\
\text { I PLACE- } \\
\text { MEMT IM } \\
\text { SPUPACE } \\
\text { (SOOO) }\end{array}$} & \multicolumn{2}{|l|}{$\begin{array}{l}\text { COSP of } \\
\text { REMONAL }\end{array}$} & \multirow{5}{*}{$\begin{array}{l}\text { POTAL } \\
\text { DIS6. AT } \\
\text { 32 พ0 IVใ }\end{array}$} & \multirow{6}{*}{$\begin{array}{c}\text { TQTAL } \\
\text { 156. } \\
52 \text { T0 } 1987 \\
\text { (19000) }\end{array}$} & \multirow{6}{*}{ 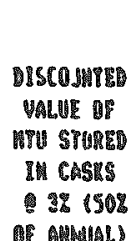 } & \multirow{6}{*}{ 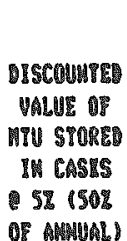 } \\
\hline & & & & & P以บ & & & & & \\
\hline & & & & & STORAGE & & & & & \\
\hline & & & & & PREPARA ILA & & & & & \\
\hline & & & & & SMIPTEMT & TOTAL & & & & \\
\hline & & & & & 180001 & $(8000)$ & $(8000)$ & & & \\
\hline
\end{tabular}

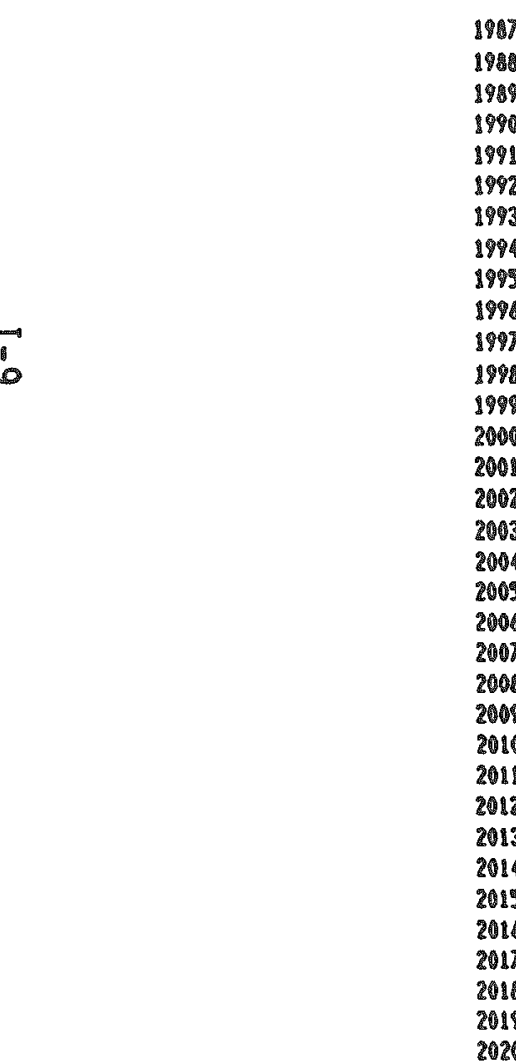

$\begin{array}{lrr}1907 & 24 & 54 \\ 1988 & 108 & 160 \\ 1999 & 163 & 323 \\ 1990 & 126 & 449 \\ 1991 & 238 & 687 \\ 1992 & 284 & 973 \\ 1993 & 258 & 1238 \\ 1994 & 428 & 1658 \\ 1985 & 365 & 2024 \\ 1996 & 454 & 2478 \\ 1997 & 516 & 2994 \\ 1998 & 189 & 3163 \\ 1999 & 272 & 3435 \\ 2000 & 390 & 3825 \\ 2001 & & \end{array}$

POTAL

PTPAL MISC M3\% MUIY COSI/S6

TOTAL IISC 152 ข้อ Cos?/re

841
4,801
6,206
5,190
9,380
10,301
9,923
15,511
12,824
16,020
10,242
6,318
9,450
13,311

7
34
46
11
75
19
72
129
109
136
157
54
82
116

3

\begin{tabular}{|c|c|}
\hline \multicolumn{2}{|l|}{940} \\
\hline & \\
\hline 5,229 & \\
\hline 9,455 & \\
\hline 10,970 & \\
\hline 10,005 & \\
\hline 15,617 & \\
\hline 13,033 & \\
\hline 16,156 & \\
\hline 18,390 & \\
\hline 6,372 & \\
\hline 9,532 & \\
\hline 13,827 & \\
\hline 3 & \\
\hline 44 & \\
\hline & \\
\hline
\end{tabular}

\begin{tabular}{|c|c|}
\hline 84 & 948 \\
\hline 4,500 & 4,414 \\
\hline 5,695 & 5,672 \\
\hline 4,794 & 4,526 \\
\hline 8,401 & 7,779 \\
\hline 9,462 & 8,585 \\
\hline 8,379 & 7,466 \\
\hline 12,123 & 11,120 \\
\hline 10,260 & 8,621 \\
\hline 12,382 & 10,414 \\
\hline 13,690 & 11,245 \\
\hline 8,600 & 3,726 \\
\hline 0,885 & $5,30 \mathrm{~A}$ \\
\hline 9,143 & 7,120 \\
\hline 2 & 2 \\
\hline 28 & 21 \\
\hline 62 & 80 \\
\hline 28 & 20 \\
\hline
\end{tabular}

12
51
77
58
108
123
108
174
144
178
192
191
95
133

20007

2000

2010

911

2013

2011

2016
2018

2013

2020

$193,813 \quad 1,157$

$13 \mathrm{~s}^{2}, 813$

110,973
073.6

921

96,406
773.8

921
7.6
798
0.8

112

190,282

112,014

1,500

1,306

$\$ 1$

776

77.92 
TALIE I- 8

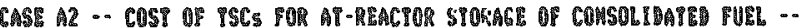

RRPOSITORY CONAFHCES OPERATIOH IN 1890

SHIPMENT IM ISC

I508 of Ar Storage Requirements Provided by Casks

\begin{tabular}{|c|c|c|c|c|c|c|c|c|c|c|}
\hline \multirow{6}{*}{ YEAR } & & \multirow{6}{*}{$\begin{array}{l}\text { MO. OP } \\
\text { MEIALL } \\
\text { CASYE }\end{array}$} & \multirow{6}{*}{$\begin{array}{l}\text { COSP OS } \\
\text { METAL } \\
\text { CASES } \\
\text { (GOOQU) }\end{array}$} & \multicolumn{3}{|c|}{$\begin{array}{l}\text { COST OB } \\
\text { ARMUAL }\end{array}$} & \multirow[b]{4}{*}{ P0IAL } & \multirow{6}{*}{ 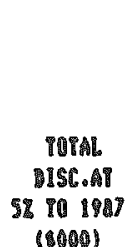 } & \multirow{6}{*}{ 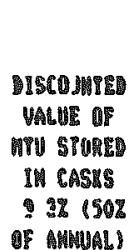 } & \multirow{6}{*}{ 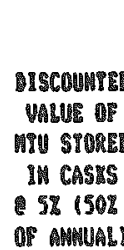 } \\
\hline & & & & $\cos 1$ & Prom & & & & & \\
\hline & & & & LOADIM & storage & & & & & \\
\hline & MOUนI PULL & & & PLACE- & PREPARATLOM & & & & & \\
\hline & STQRE) & & & MENIIN & 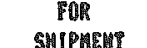 & & ISC. AT & & & \\
\hline & AพพับAL CUMULATIUอ & & & $\begin{array}{l}3010008 \\
(8000)\end{array}$ & $\begin{array}{l}\text { SHIPNET } \\
(8000)\end{array}$ & $(\$ 000)$ & $\begin{array}{l}318011808 \\
(10001\end{array}$ & & & \\
\hline
\end{tabular}

\begin{tabular}{|c|c|c|c|c|c|c|c|c|c|c|c|}
\hline 1987 & 24 & 54 & 1 & 911 & 7 & & 948 & 946 & 980 & 12 & 12 \\
\hline 1988 & 108 & 180 & 3 & 2,760 & 20 & & 2,780 & 2,690 & 2,848 & 51 & 50 \\
\hline 1989 & 183 & 323 & 4 & 3,600 & 27 & & 3,027 & 3,418 & 3,290 & $\pi$ & 74 \\
\hline 1990 & 126 & 449 & 3 & 2,649 & 2) & & 2,640 & 2,443 & 2,300 & 50 & 54 \\
\hline 1991 & 233 & 687 & 1 & 5,226 & 40 & & 5,268 & 4,879 & 4,332 & 108 & 96 \\
\hline 1912 & 28 & 973 & 1 & $b_{9} 135$ & 53 & & 8,908 & 5,800 & 5,414 & 123 & 112 \\
\hline 1993 & 256 & 1231 & 7 & 5,915 & 47 & & 5,992 & 4,993 & 4,449 & 100 & 18 \\
\hline 1994 & 428 & 1459 & 12 & 10,020 & 80 & & 10,100 & 8,312 & 7,170 & 178 & 152 \\
\hline 1905 & 365 & 2024 & 10 & 0,260 & 57 & & 8,327 & 6,573 & $5,83.3$ & 144 & 124 \\
\hline 1998 & 1554 & 2478 & 12 & 9,828 & 30 & & 8,908 & 7,504 & 0,387 & 174 & 146 \\
\hline 1997 & 516 & 2994 & 11 & 11,368 & 13 & & 11,481 & 0,521 & 7,030 & 112 & 158 \\
\hline 1998 & 169 & 3183 & 5 & 4,035 & 33 & & 1,060 & 2,939 & 2,379 & 61 & 49 \\
\hline 1999 & 272 & 3435 & $y$ & 5,628 & 47 & & 9,875 & 3,410 & 3,180 & 95 & 76 \\
\hline 2000 & 390 & 3825 & 11 & 8,818 & 73 & & , 804 & 0,050 & 4,712 & 133 & 103 \\
\hline 2001 & & & (2) & & & 2 & 2 & 1 & 1 & & \\
\hline 2002 & & & 1241 & & & 27 & 27 & 17 & 13 & & \\
\hline 2003 & & & (54) & & & 80 & 60 & ฟ & 23 & & \\
\hline 2004 & & & 1251 & & & 28 & 20 & 17 & 12 & & \\
\hline
\end{tabular}

TอนAL

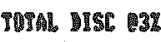

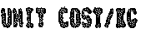

T0IM IISG

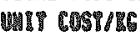

193

\begin{tabular}{|c|c|}
\hline 95,197 & 897 \\
\hline 61970 & 596 \\
\hline 195. & 1.4 \\
\hline 59,400 & $4 / 2$ \\
\hline
\end{tabular}

เงรี.

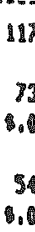

$117 \%$

8,089

59,926

1,500

1,306

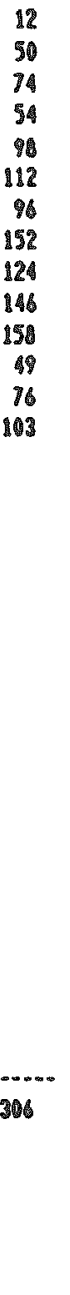


TALL $1-7$

CASP A3 - COSY OR SUCS FOR AI-REACTUR STOKAGE OP IMTACT PUEL ASSEMLIES -.

REPOSITORY COMWEMCES OPERATIOH IN 1996

SHIPHPUI IM DOE TRAMEPIST CASI

(50\% of AR Storage Requsrements Provided by Cashs)

\begin{tabular}{|c|c|c|c|c|c|c|c|c|c|c|}
\hline \multirow[b]{3}{*}{ YEAT } & & \multirow{3}{*}{$\begin{array}{l}\text { WD, of } \\
\text { AEIAL } \\
\text { Co: }\end{array}$} & \multirow{3}{*}{$\begin{array}{l}\text { COSP OP } \\
\text { METIAL } \\
\text { CASES }\end{array}$} & \multicolumn{3}{|c|}{$\begin{array}{l}\text { COST OP } \\
\text { REMQVAL }\end{array}$} & \multirow{3}{*}{$\begin{array}{c}\text { Torat } \\
\text { MISC. Ar } \\
\text { 3\% } 90 \text { 1887 }\end{array}$} & \multirow{4}{*}{$\begin{array}{c}\text { 90TAL } \\
\text { 15C.AT } \\
\text { 52 10 1987 } \\
\text { (9000) }\end{array}$} & \multirow{4}{*}{ 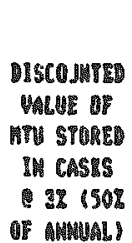 } & \multirow{4}{*}{ 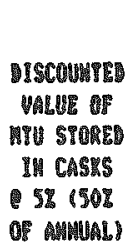 } \\
\hline & 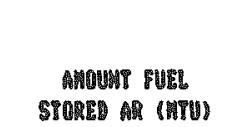 & & & $\begin{array}{l}\text { CAST aE } \\
\text { LOAEIME } \\
\text { PLACE- } \\
\text { MEWT IK }\end{array}$ & 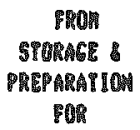 & & & & & \\
\hline & 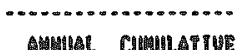 & & & STORAGE & SHIPUEMI & IOPAL & & & & \\
\hline & 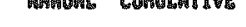 & & & & (8000) & & & & & \\
\hline
\end{tabular}

1907
1998
1989
1990
1991
1992
1993
1994
1995
1998
1997
1941
1999
2000
2001
2002
2003
2004
2005
2004
2007
2008
2009
2010
2011
2012
2013
2014
2015
2016
2017
2018
2018
2020

\begin{tabular}{|c|c|c|c|c|c|c|c|c|}
\hline 24 & 54 & 1 & 856 & 8 & & 963 & 963 & 963 \\
\hline 108 & 160 & 5 & 4,780 & 34 & & $\$, 114$ & 4,674 & 1,505 \\
\hline 163 & 923 & 7 & 5,516 & 80 & & 5,564 & 3,244 & 5,048 \\
\hline 126 & 448 & $s$ & 8,620 & 41 & & 1,681 & 4,265 & 1,028 \\
\hline 238 & 687 & II & 0,338 & 75 & & 8,813 & 7,875 & 8,021 \\
\hline 286 & 973 & 13 & 9,672 & 80 & & 18780 & 8,418 & 7,648 \\
\hline 258 & 1231 & 12 & 8,820 & 22 & & 8.902 & 7,455 & $\$, 643$ \\
\hline 128 & 1458 & 19 & 13,794 & 129 & & 13,223 & 18,321 & 9,095 \\
\hline 365 & 2024 & 16 & 11,408 & 100 & & 11,507 & 0,158 & 7,040 \\
\hline 454 & 2478 & 20 & 14,240 & 136 & & 14,378 & 11,018 & 9,267 \\
\hline 516 & 2994 & 23 & 16,215 & 157 & & 16,372 & 12,1102 & 10,051 \\
\hline 169 & 3163 & 8 & 5,616 & 34 & & 5,670 & 4,008 & 39,318 \\
\hline 272 & 3435 & 12 & 89800 & 2 & & 8482 & 3,948 & 4,723 \\
\hline \multirow[t]{5}{*}{390} & 3825 & 17 & 11,832 & IIS & & 11,948 & 0,138 & 6,338 \\
\hline & & 131 & & & 84 & 34 & 55 & 42 \\
\hline & & (39) & & & 1,080 & 1,088 & 698 & 523 \\
\hline & & $(209)$ & & & 2,183 & 2,403 & 1,547 & 1,136 \\
\hline & & (41) & & & 1,144 & 1,144 & 692 & 498 \\
\hline
\end{tabular}

บของ

THYM MIEF E3\% แW IT COST/RG

TORA MISE 05 WHIP COSP/R6

\begin{tabular}{|c|c|c|}
\hline 124,297 & 1,157 & $4_{1} 79$ \\
\hline 99,432 & 921 & 2,909 \\
\hline 355.9 & $\$ .8$ & 12.0 \\
\hline $8 S_{0} 478$ & 798 & 2,202 \\
\hline 886.2 & 8.6 & 11.8 \\
\hline
\end{tabular}

130,243

103,34

89,870

1,500 
IABLE I-

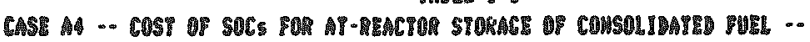

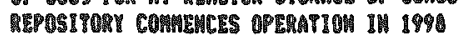

SHPMEMP IM DOE IRAMSPORI CASI

$$
\text { CAPACITY - 124/52 }
$$

1504 of Storage Requipenemes Provided Cashs)

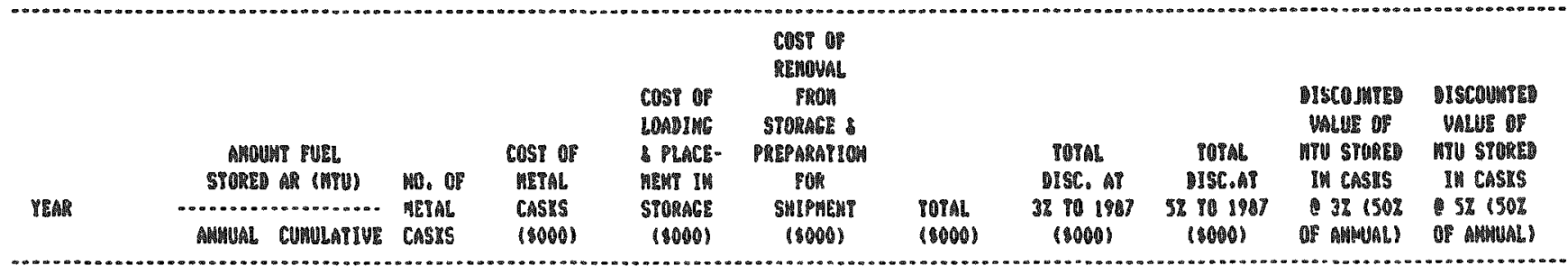

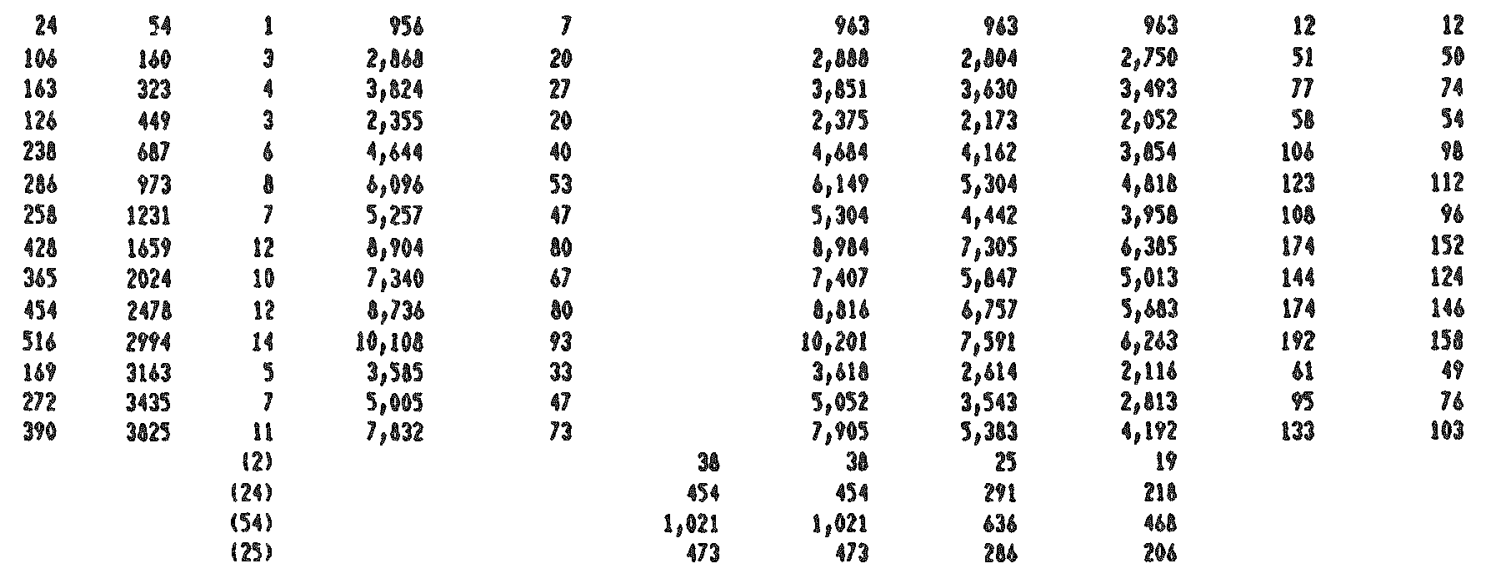

รอบดเ

1987
1980
1909
1990
1991
1992
1993
1994
1995
1998
1997
1990
1998
2000
2001
2002
2003
2004
2005
2008
2007
2008
2009
2010
2011
2012
2013
2014
2015
2016
2018
2018
2019
2020

TORML ISE AOS

UMIT COSP/RG

TดPAL MISE UHIP COST/2

\begin{tabular}{|c|c|c|c|c|c|c|c|}
\hline 77,510 & 67 & 1,985 & 00,112 & 03,755 & 55,262 & 1,500 & 1,306 \\
\hline 81,972 & 548 & 1,238 & & 63,755 & & & \\
\hline 41.1 & 8.4 & 8.8 & & 12.3 & & & \\
\hline 53,879 & 472 & 911 & & & 55,262 & & \\
\hline$\$ 11.3$ & 8.01 & 9.7 & & & 142.3 & & \\
\hline
\end{tabular}


TAELE I-9

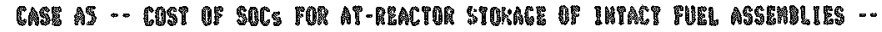

REYOSITSOY COMMENCES OPERATIOU IN 1998

OHE-TIME USE OP SOC POR SMIPMEN? CAPACITY - (20/5?)

150\% of AR Storage Requirenents Provided My Casts)

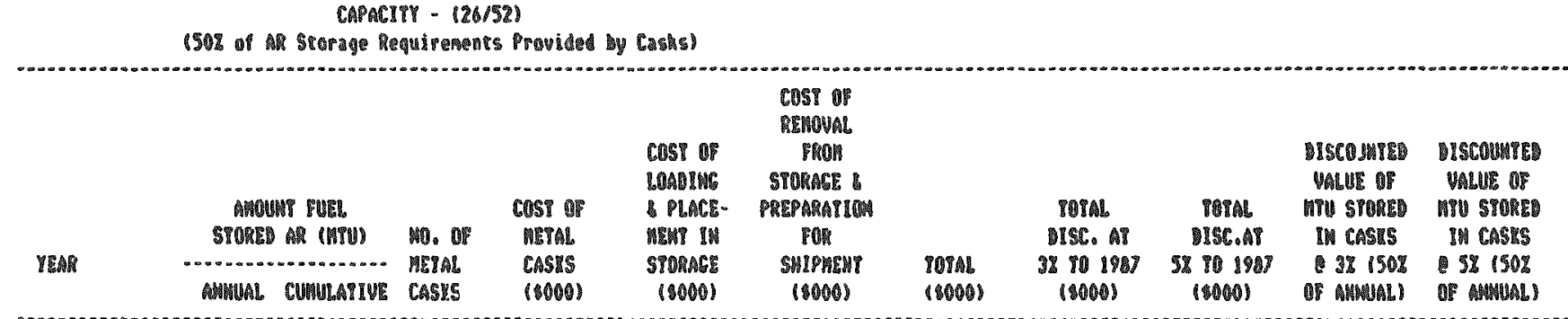

\begin{tabular}{|c|c|c|c|c|c|c|c|c|c|c|c|}
\hline 1987 & 24 & 54 & 1 & 956 & 7 & & 983 & 963 & 963 & 12 & 12 \\
\hline 1896 & 108 & 160 & 5 & 4,780 & 34 & & 8,814 & 4,674 & 8505 & $\$ 1$ & 50 \\
\hline 1919 & 163 & 323 & 7 & 5,516 & 48 & & 5,584 & 5,244 & 5,048 & 37 & 74 \\
\hline 1990 & 128 & 449 & 6 & 1,620 & 41 & & 4,881 & 9,265 & 1,026 & 58 & 54 \\
\hline 1991 & 238 & 687 & 11 & 338 & 75 & & 8,413 & 7,175 & 6,921 & 104 & 96 \\
\hline 1992 & 288 & 873 & 13 & 8,872 & 8 & & 9,760 & 8,818 & 7,648 & 123 & 112 \\
\hline 1993 & 258 & 1231 & 18 & 6,820 & 12 & & 8,902 & 7,455 & 6,613 & 100 & 86 \\
\hline 1994 & 428 & 1858 & 18 & 13,794 & 129 & & 13,923 & 11,321 & 9,395 & 174 & 152 \\
\hline 1995 & 365 & 2024 & 16 & 11,488 & 109 & & 11,597 & 8,155 & 7,099 & 148 & 124 \\
\hline 1994 & 454 & 2478 & 20 & 14,240 & 136 & & 10,376 & 11,018 & 9267 & 174 & 148 \\
\hline 1897 & 516 & 2994 & 23 & 16,215 & 157 & & 16,372 & 12,102 & 10,051 & 192 & 158 \\
\hline 1998 & 160 & 3163 & 1 & 5,616 & 58 & & 5,870 & 4,098 & 3,315 & 81 & 49 \\
\hline 1899 & 272 & 3435 & 12 & 8,400 & 18 & & 8,182 & 8,910 & 4,723 & 85 & 78 \\
\hline 2000 & 390 & 3825 & 17 & 11,832 & 118 & & 11,948 & 8,138 & 1,336 & 133 & 203 \\
\hline 2008 & & & (3) & & & 3 & 3 & 2 & 2 & & \\
\hline 2002 & & & (39) & & & ఫీర్ & 4 & 28 & 21 & & \\
\hline 2003 & & & (89) & & & 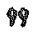 & 99 & 42 & 48 & & \\
\hline 2004 & & & (41) & & & 6 & 96 & 28 & 20 & & \\
\hline
\end{tabular}

P0Tล

TORAL OISC UसLI COST/RG

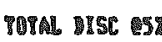
แHIY COST/RG
170

124,237

124,298

98,392
$\$ 45.8$

1,157

86,470

S18.2

921
7.6

788
125,836

100,972

07,358

1,500
100,472
$\square 66.6$

07.358 
TABLE 1-10

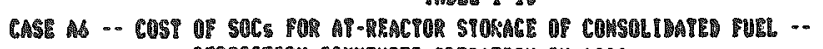

MOSITORY COMAEMCES OPERATIOH IN 1998

$$
\text { CAPACITY - } 126 / 521
$$

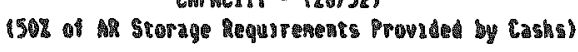

\begin{tabular}{|c|c|c|c|c|c|c|c|c|c|c|}
\hline \multirow[b]{4}{*}{ YEAT } & \multirow{4}{*}{\multicolumn{2}{|c|}{ 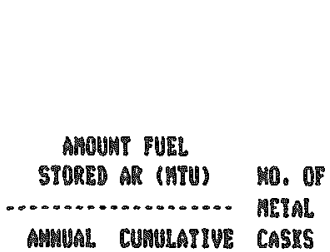 }} & \multirow{4}{*}{$\begin{array}{l}\text { COSI OF } \\
\text { MEIML } \\
\text { CASUS } \\
\text { PSOOOH }\end{array}$} & \multicolumn{3}{|c|}{$\begin{array}{l}\text { COSP OP } \\
\text { REMOUAL }\end{array}$} & & & & \\
\hline & & & & $\cos T^{\circ}$ of & Prom & & & & DISCOHWTI & IsCOUMIE \\
\hline & & & & $\begin{array}{l}\text { LOADING } \\
\text { \& PLACE- } \\
\text { MEN IN }\end{array}$ & $\begin{array}{l}\text { SPQRAGE } \\
\text { PREPARATIOA } \\
\text { POR }\end{array}$ & & $\begin{array}{l}\text { PUTAL } \\
\text { DISC。 AI }\end{array}$ & $\begin{array}{c}\text { POPAL } \\
\text { IISC.A? }\end{array}$ & $\begin{array}{l}\text { VALUE OP } \\
\text { MTU SPURE } \\
\text { IN CASES }\end{array}$ & $\begin{array}{l}\text { VALUE OF } \\
\text { MTU STORE } \\
\text { II CASES }\end{array}$ \\
\hline & & & & $\begin{array}{r}\text { STORACE } \\
(\text { SO00) }\end{array}$ & $\begin{array}{l}\text { SHIPFEMT } \\
\text { (10000) }\end{array}$ & $\begin{array}{l}\text { P0IAL } \\
\text { P(1000) }\end{array}$ & $\begin{array}{c}38101987 \\
100001\end{array}$ & $\begin{array}{c}58901987 \\
(6000)\end{array}$ & 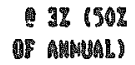 & $\begin{array}{l}\text { I2 (50Z } \\
\text { OF MANUAL) }\end{array}$ \\
\hline
\end{tabular}

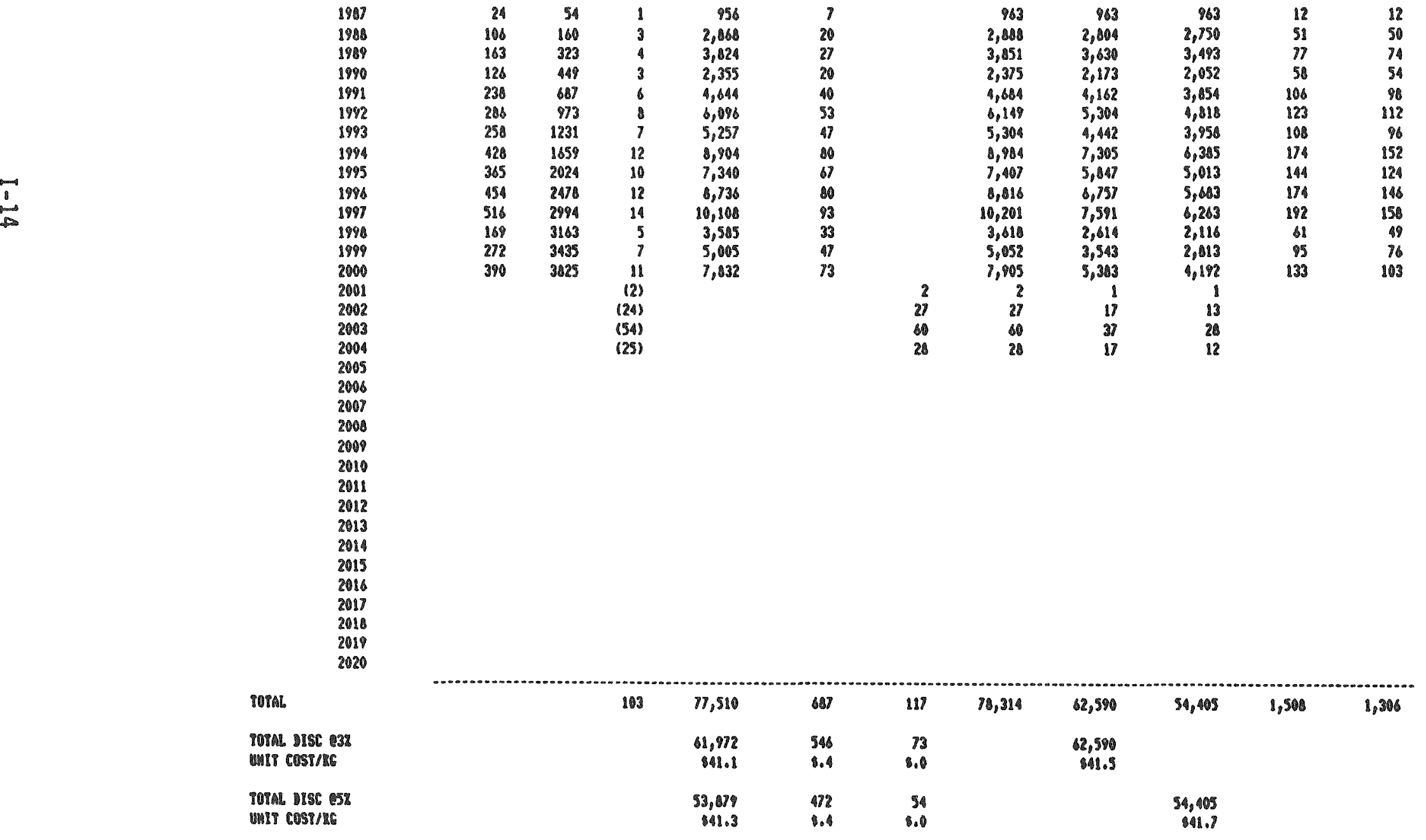


TABLE 1-11

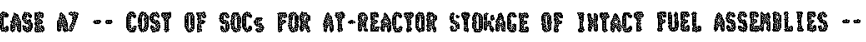

REPOSITORY COHMLHCES OFERATIOM IM 1998

SHIPATHI IN OUERPACEE SOC

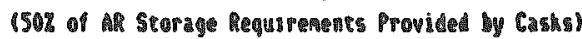

\begin{tabular}{|c|c|c|c|c|c|c|c|c|c|c|}
\hline \multirow[b]{3}{*}{ rean } & \multirow{3}{*}{\multicolumn{2}{|c|}{ 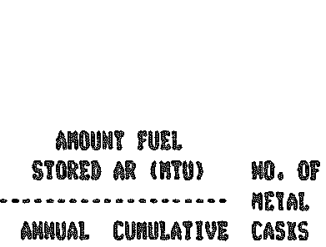 }} & \multirow{3}{*}{$\begin{array}{l}\text { COST OH } \\
\text { METAL } \\
\text { CASES } \\
\text { (\$OOO) }\end{array}$} & \multirow{3}{*}{ 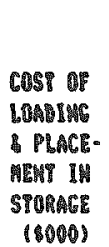 } & \multicolumn{2}{|l|}{$\begin{array}{l}\text { COST OP } \\
\text { REMOUAL }\end{array}$} & \multirow{2}{*}{ 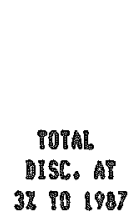 } & \multirow{3}{*}{$\begin{array}{c}\text { TOTAL } \\
\text { ISC.AT } \\
\text { S2 TO } 1987 \\
\text { (1900) }\end{array}$} & \multirow{3}{*}{ 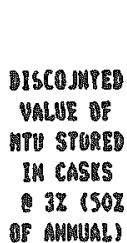 } & \multirow{3}{*}{ 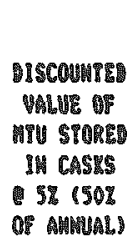 } \\
\hline & & & & & 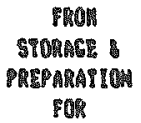 & & & & & \\
\hline & & & & & $\begin{array}{c}\text { SHIP以ERT } \\
\text { (S000) }\end{array}$ & $\begin{array}{l}\text { I0TAL } \\
19000)\end{array}$ & $\begin{array}{c}\text { 31 } 101947 \\
(1000)\end{array}$ & & & \\
\hline
\end{tabular}

\begin{tabular}{|c|c|c|c|c|c|c|c|c|c|c|}
\hline 24 & 54 & 1 & 856 & 7 & & 963 & 983 & 983 & 12 & 12 \\
\hline 106 & 100 & 5 & 4,700 & 34 & & 4,814 & 4,674 & 4,585 & 51 & 50 \\
\hline 163 & 323 & $?$ & 5,516 & 88 & & 5,564 & 5,240 & 5,040 & $n 7$ & 71 \\
\hline 128 & 448 & 6 & 4,820 & 41 & & $\triangle, B A 1$ & 4,265 & 4,026 & 58 & 54 \\
\hline 238 & 697 & 11 & 1336 & 75 & & 0,413 & 7,475 & 6,021 & 106 & 90 \\
\hline 288 & 973 & 13 & 9,872 & 8 & & 9,760 & 8,410 & 7,648 & 123 & 112 \\
\hline 258 & 1231 & 12 & 8,180 & 82 & & 0,902 & 7,455 & 6,843 & 108 & 96 \\
\hline 428 & 1658 & 19 & 13,794 & 129 & & 13,923 & 11,321 & 9,685 & 178 & 152 \\
\hline 365 & 2024 & 16 & 11,480 & 109 & & 11,597 & 0,155 & 8,848 & 164 & 124 \\
\hline 454 & 2078 & 20 & 14,200 & 138 & & 14,376 & 11,010 & 9,267 & 174 & 196 \\
\hline 516 & 2994 & 23 & 16,215 & 157 & & 16,372 & 12,182 & 10,051 & 192 & 150 \\
\hline 169 & 3163 & a & 5,816 & 54 & & 5,670 & 4,096 & 3,315 & 61 & 99 \\
\hline 272 & 3435 & 12 & 8,400 & 82 & & 1,462 & 5,949 & 4,723 & 95 & 76 \\
\hline \multirow[t]{5}{*}{390} & 3825 & 17 & 11,832 & 118 & & 11,9818 & 8,136 & 6,336 & 133 & 103 \\
\hline & & (3) & & & 21 & 21 & 14 & 10 & & \\
\hline & & (39) & & & 270 & 270 & 173 & 130 & & \\
\hline & & $(89)$ & & & 816 & 816 & 364 & 282 & & \\
\hline & & (61) & & & 284 & 284 & 172 & 124 & & \\
\hline
\end{tabular}

1987
1988
1909
1990
1991
1902
1993
1994
1995
1998
1997
1998
1998
2000
2001
2002
2003
2004
2005
2008
2007
2008
2008
2010
2011
2012
2013
2014
2015
2018
2017
2018
2018
2020

TOPA ISE Q3Z

UMIY COS?

POTAL MISC E5\% ต418 cos?/RE

\begin{tabular}{|c|c|c|c|c|c|c|c|}
\hline 124,287 & 1,157 & $I_{8} 140$ & 126,634 & 101,695 & 97,814 & 1,500 & 1,308 \\
\hline $99,4,32$ & 921 & 742 & & 101,085 & & & \\
\hline 45.9 & 100 & 8.5 & & $\$ 87.0$ & & & \\
\hline 86,470 & 798 & 548 & & & 37,810 & & \\
\hline 184.2 & 8.8 & 8.4 & & & 887.2 & & \\
\hline
\end{tabular}


TALL $1-12$

CASE AO -- COST OP SOCS POR AP-REACTOR SPOKAGE DF COMSOLIDATR PUEL -

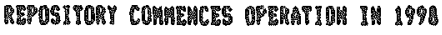

SHIPHARI IN OUERPACHED SOC

1505 of ar storage Requireneats Provided Dy Cashs) 
TAMLE I-13

CASE 1 - COST

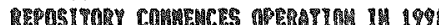

SMIPMEET IM TSE
CAPACITY - I32/78

(503 of aR Storage Requerevemes Provided by Cashs)

\begin{tabular}{|c|c|c|c|c|c|c|c|c|c|c|}
\hline \multirow{7}{*}{ VEAR } & & \multirow{6}{*}{$\begin{array}{l}\text { MO. OP } \\
\text { me }\end{array}$} & \multirow{6}{*}{$\begin{array}{l}\text { COST OP } \\
\text { MLETAL } \\
\text { CASPS }\end{array}$} & \multicolumn{3}{|c|}{ 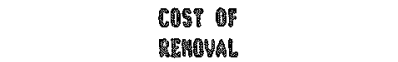 } & \multirow{6}{*}{$\begin{array}{l}\text { TOTML } \\
\text { IISC. AI } \\
\text { 3. PO I9O }\end{array}$} & \multirow{6}{*}{$\begin{array}{l}\text { POFAL } \\
\text { DISC.AIT } \\
\text { 5E YO IOS7 }\end{array}$} & \multirow{7}{*}{ 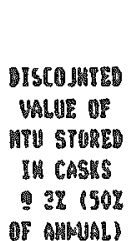 } & \multirow{7}{*}{ 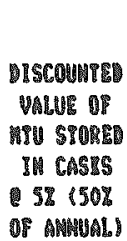 } \\
\hline & & & & cos? of & FROA & & & & & \\
\hline & & & & LOADIMG & STOMAGE \& & & & & & \\
\hline & 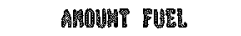 & & & 8LACE: & PREPARATIOA & & & & & \\
\hline & STORED & & & ME⿵冂卄 II & Pon & & & & & \\
\hline & 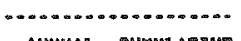 & & & STORAGE & SHIPМЁ? & rotal & & & & \\
\hline & ANMUAL CUMULATIUE & & 180001 & $(8000)$ & 190001 & $(8000)$ & $(8000)$ & $(9000)$ & & \\
\hline
\end{tabular}

\begin{tabular}{|c|c|c|c|c|c|c|c|c|c|c|c|}
\hline 1987 & 24 & 54 & 1 & 941 & 8 & & 908 & 90 & 948 & 12 & 12 \\
\hline 1988 & 108 & 160 & 4 & 3,681 & 30 & & 3,711 & $3_{8} 603$ & 3,534 & 51 & 50 \\
\hline 1989 & 183 & 323 & 6 & 5,346 & 45 & & 5,391 & 5,002 & 4,800 & 78 & 74 \\
\hline 1990 & 128 & 448 & 1 & 3,697 & 30 & & 3,527 & 3,228 & 3,047 & 51 & 54 \\
\hline 1991 & 231 & 607 & (1) & 6,085 & 60 & & 6,945 & 8,171 & 5,714 & 106 & 98 \\
\hline 1992 & 288 & 973 & 10 & 8,800 & 75 & & 8,535 & 7,363 & 8,688 & 123 & 112 \\
\hline 1993 & 258 & $123 !$ & 9 & 7,523 & ๘6 & & 7,591 & 6,357 & 5,864 & 108 & 96 \\
\hline 1994 & 428 & 1659 & 15 & 12,003 & 113 & & 12,516 & 10,177 & 0,045 & 174 & 152 \\
\hline 1995 & 365 & 2024 & 13 & 10,610 & 98 & & 10,716 & 8,458 & 7,853 & 144 & 124 \\
\hline 1996 & 454 & 2478 & 16 & 12,960 & 120 & & 13,080 & 10,028 & 8,432 & 174 & 146 \\
\hline 1997 & 518 & 299 & 18 & 14,438 & 135 & & 14,573 & 10,844 & 8,947 & 192 & 158 \\
\hline 1998 & 169 & 3163 & 6 & 4,796 & 45 & & 4,831 & 3,490 & 2,825 & 81 & 49 \\
\hline 1999 & 272 & 3435 & 9 & 7,158 & 68 & & 7,226 & 3.060 & 4,024 & 85 & 76 \\
\hline 2000 & 390 & 3825 & 13 & 10,296 & 98 & & 10,384 & 7,078 & 5,512 & 133 & 103 \\
\hline 2001 & & & 12) & & & 2 & 2 & 2 & 1 & & \\
\hline 2002 & & & 1301 & & & 35 & 35 & 22 & 1) & & \\
\hline 2003 & & & (89) & & & 30 & 80 & 50 & 37 & & \\
\hline 2004 & & & 1311 & & & 36 & 36 & 22 & 16 & & \\
\hline
\end{tabular}

PETAL MIS

WMIT COST/EG

POTAL. MISG

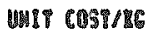

36

2006

2007

2000

2009

2010

2012

2013

2015

2016

2017

2018

2020

(10)

132

108,992

100,919

87,101
857.7

75,487

350.0

993

153

110,138

791
0.5
0.5

45

8.1

70
$76,442 \quad 1,506 \quad 1,306$

9787
958.3

76,942 
IABLE I-10

CASE 22 - COST OP ISCS POR AT-REACTOR STOLGGE OF COASOLIDAPE PUEL -.

REPOSI TORY COMMEHCES ORRATIOW IM 1998

$$
\text { SHIPHEMT IH TSE }
$$

(50\% of An Storag̨e heguireneats Provided by Cashs)

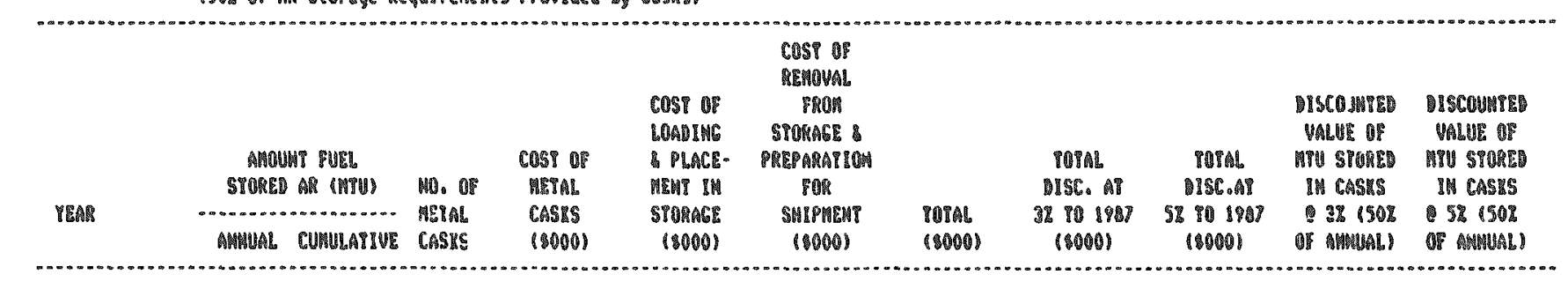

\begin{tabular}{|c|c|c|c|c|c|c|c|c|c|c|c|}
\hline 1987 & 24 & 54 & 1 & 941 & 7 & & 948 & 848 & 948 & 12 & 12 \\
\hline 1980 & 106 & 160 & ? & 1,058 & 15 & & 1,873 & 1,818 & 1,784 & 51 & 50 \\
\hline 1989 & 163 & 323 & 3 & 2,715 & 22 & & 2,737 & 2,580 & 2,483 & $n$ & 74 \\
\hline 1990 & 126 & 449 & 3 & 2,673 & 22 & & 2,695 & 2,467 & 2,328 & 58 & $\$ 4$ \\
\hline 1991 & 230 & 687 & 5 & 4,305 & 37 & & 4,422 & 3,924 & 3,630 & 106 & 96 \\
\hline 1092 & 284 & 873 & 6 & 5,104 & 45 & & 5,228 & 4,510 & 9,087 & 223 & 112 \\
\hline 1993 & 250 & 1231 & 5 & $\$, 270$ & 37 & & 4,307 & 3,607 & 3,214 & 108 & 96 \\
\hline 1994 & 428 & 1859 & 8 & 7,596 & 67 & & 1,063 & 1,231 & 3,446 & 174 & 152 \\
\hline 1995 & 365 & 2024 & 8 & 8,600 & 60 & & 8,740 & 5,320 & 1,562 & 184 & 124 \\
\hline 1988 & 454 & 2478 & $\vartheta$ & 7,452 & 47 & & 7,519 & 5,763 & 4,848 & 174 & 148 \\
\hline 1997 & 516 & 2094 & 18 & 9,031 & 82 & & 9,113 & 6,781 & 5,595 & 192 & 158 \\
\hline 1988 & 169 & 3163 & 4 & 3,264 & 30 & & 3,294 & 2,380 & 1, 1,26 & 81 & 49 \\
\hline 1989 & 272 & 3435 & 6 & $A_{f} 804$ & 45 & & 4,929 & 3,457 & 2,744 & 95 & 76 \\
\hline 2000 & 390 & 3825 & 8 & 8,410 & 60 & & 6,550 & 4,453 & 3,460 & 133 & 103 \\
\hline 2001 & & & (1) & & & 1 & 1 & 1 & 1 & & \\
\hline 2002 & & & (18) & & & 22 & 22 & 14 & 10 & & \\
\hline 2003 & & & (41) & & & 49 & 48 & 31 & 23 & & \\
\hline 2004 & & & (188) & & & 23 & 23 & 14 & 10 & & \\
\hline
\end{tabular}

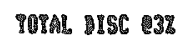
แพIT Cosise

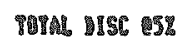

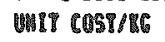

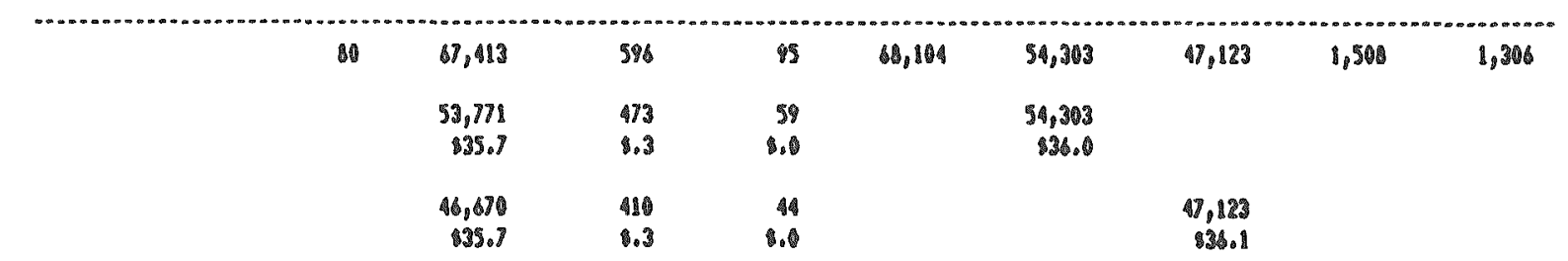


TABLE 1-15

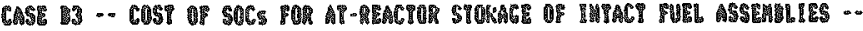

MEPDS ITOAY COMHEMCES OPRRATION IN 1988

SMIPHEHI IM DOE IRAMSPOR CASE

$$
\text { CAPACITY - } 122 / 761
$$

(50) of af Storage Requireneats Provided by Cashs)

\begin{tabular}{|c|c|c|c|c|c|c|c|c|c|c|}
\hline \multirow[b]{4}{*}{ Vrar } & & \multirow{4}{*}{$\begin{array}{l}\text { Mo. OF } \\
\text { MELAL } \\
\text { CASKS }\end{array}$} & \multirow{4}{*}{ 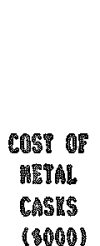 } & \multicolumn{3}{|c|}{$\begin{array}{l}\text { COST OF } \\
\text { REROVAL }\end{array}$} & & & & \\
\hline & & & & $\begin{array}{l}\text { COST OP } \\
\text { LOABIAC } \\
\text { \& PLACE- }\end{array}$ & 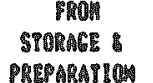 & & & & 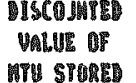 & $\begin{array}{l}\text { MISCONWYIS } \\
\text { UALUE OF } \\
\text { WTU STORES }\end{array}$ \\
\hline & $\begin{array}{l}\text { ANWET } \\
\text { STORED }\end{array}$ & & & MEMT IM & 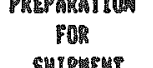 & & $\begin{array}{l}\text { IUtab } \\
\text { DISC. }\end{array}$ & IISCAI & III CASES & II CASES \\
\hline & 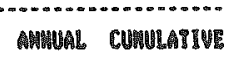 & & & $\begin{array}{l}\text { STORACE } \\
\text { ( } 2000)\end{array}$ & $\begin{array}{l}\text { ShIPWeme } \\
\text { (10000) }\end{array}$ & $\begin{array}{l}100 \mathrm{PL} \\
(8000)\end{array}$ & $\begin{array}{c}312901987 \\
(2000)\end{array}$ & $\begin{array}{c}5 \% \text { If } 1977 \\
(10000)\end{array}$ & 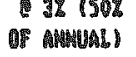 & 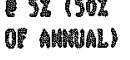 \\
\hline
\end{tabular}

\begin{tabular}{|c|c|c|c|c|c|c|c|c|c|c|c|}
\hline 1987 & 24 & 54 & 1 & 858 & 1 & & 984 & 984 & 16 & 12 & 12 \\
\hline 1988 & 106 & 180 & 4 & 3,124 & 30 & & 3,155 & 3,712 & 3,871 & 51 & 50 \\
\hline 1989 & 183 & 923 & 6 & 5,223 & 45 & & 5,268 & 1,866 & 4,7776 & $\pi$ & 74 \\
\hline 1990 & 126 & 448 & 4 & 3,100 & 30 & & 3,138 & 2,872 & 2,711 & 38 & 54 \\
\hline 1991 & 238 & 607 & 1 & 0,120 & so & & 8,100 & 5,491 & 5,084 & 108 & 9 \\
\hline 1992 & 286 & 973 & 10 & 7,520 & 75 & & 7,595 & 1,552 & 5,951 & 123 & 112 \\
\hline 1903 & 258 & 1231 & 9 & 6,867 & 68 & & 6,755 & 5,657 & 5,040 & 108 & 96 \\
\hline 1994 & 428 & 1659 & 15 & 11,025 & 113 & & 11,121 & 9,056 & 7,015 & 174 & 152 \\
\hline 1995 & 385 & 2024 & 13 & 9,430 & 98 & & 1,536 & 7,520 & 6,454 & 144 & 124 \\
\hline 1996 & 454 & 2878 & 16 & 11,520 & 120 & & 11,640 & 8,21 & 7,503 & $18 \%$ & 146 \\
\hline 1997 & 516 & 2984 & if & 12,834 & 135 & & 12,869 & 1,850 & 7,962 & 192 & 158 \\
\hline 189 & 188 & 3183 & $b$ & 4,254 & 85 & & 1,299 & 3,100 & 2,318 & (1) & 49 \\
\hline 1999 & 272 & 3435 & 9 & 0,363 & 68 & & 6, กง1 & 7.,510 & 3,581 & 95 & 76 \\
\hline 2000 & 390 & 3925 & 13 & 1,152 & 8 & & 9,250 & 5,298 & 4,905 & 133 & 103 \\
\hline 2008 & & & (2) & & & 8 & 10 & 46 & 35 & & \\
\hline 2002 & & & $130)$ & & & 1,042 & 1,042 & 880 & 501 & & \\
\hline 2003 & & & $(89)$ & & & 2,398 & 2,396 & 1,483 & 1,098 & & \\
\hline 2004 & & & (31) & & & $1,07 n$ & 1,077 & 651 & 970 & & \\
\hline
\end{tabular}

TOYA MIS ๕2ม UมII COSP/RG

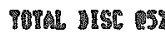
4มIT COST/R

\begin{tabular}{|c|c|c|c|c|c|c|c|c|}
\hline 132 & 96,024 & 993 & 1,584 & 1093801 & 22,172 & 71,138 & 1,500 & 1,908 \\
\hline & 70,522 & 791 & 2,858 & & 2,172 & & & \\
\hline & $\$ 52.1$ & 85 & 11.8 & & 554.5 & & & \\
\hline & 80,348 & 685 & 20104 & & & 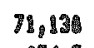 & & \\
\hline & 552.3 & 8.5 & 11.8 & & & (54, os & & \\
\hline
\end{tabular}


TAELE I-16

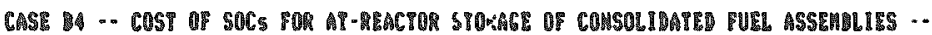

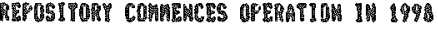

SHIPMEHT IVI DOE PRAMEPURT CAST

CAPACITY - $(32 / 76)$

1502 of AR Sorage Requirenents Provided by Caskg)

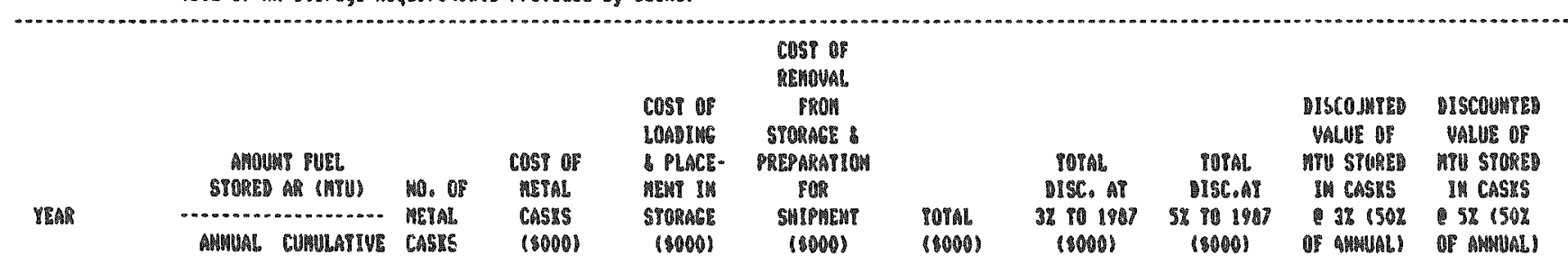

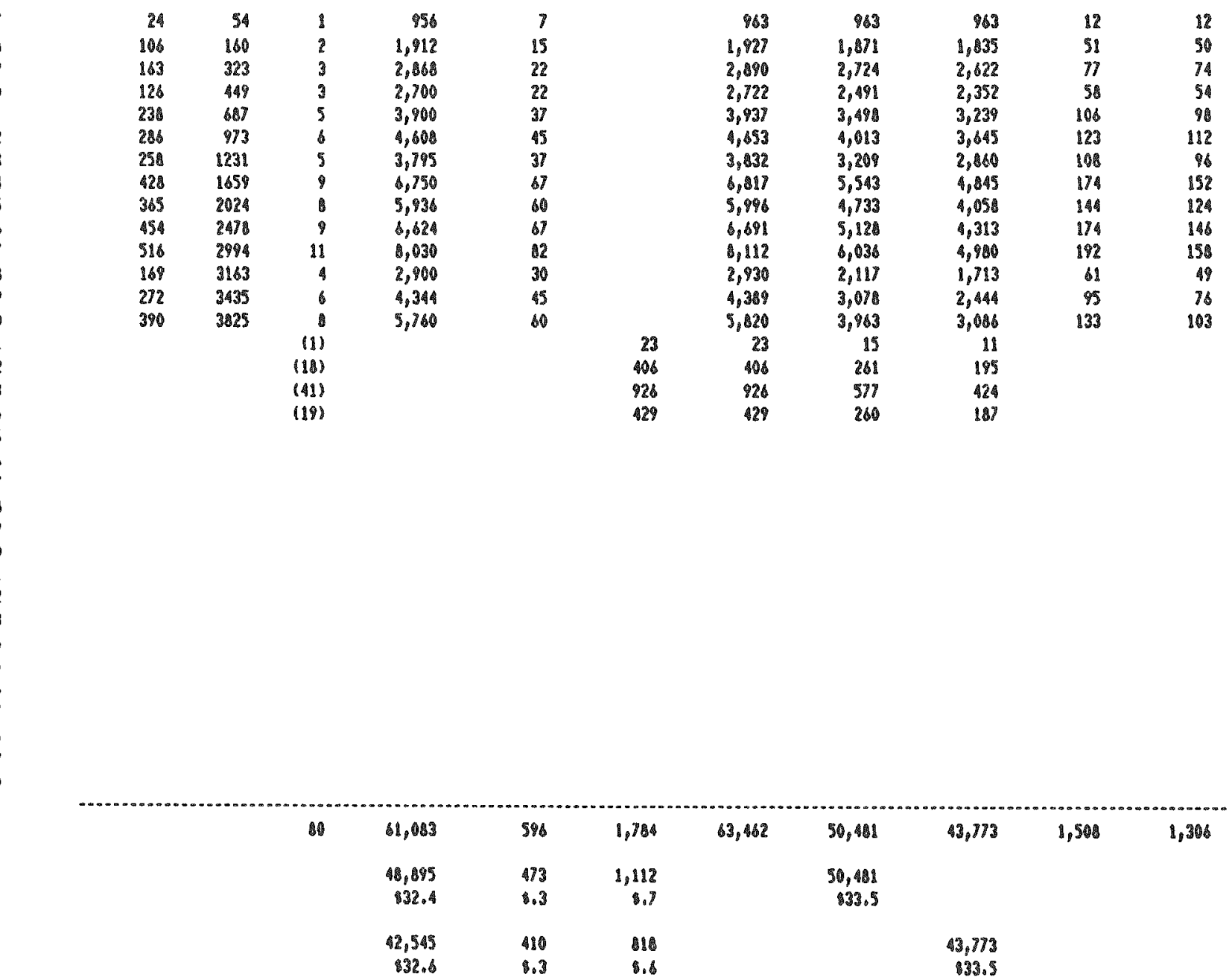

TอใAใ

TOPAL IISC BZ

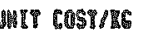

TORAL IISC Q5\% UมIT COST/IC
1907
1988
1989
1990
1991
1992
1913
1994
1995
1996
1997
1998
1999
2000
2001
2002
2003
2004
2005
2006
2007
2000
2009
2010
2011
2012
2013
2014
2015
2016
2017
2018
2019
2020

(1000)

(8000)

(5000) 
TARLE 1-17

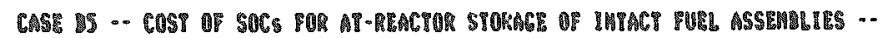

REPOSI YORY COWHEMCES OPERATIOM IM 1 HOS

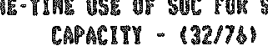

1502 of Ari Storage Requirenears Proviand by Cashs!

\begin{tabular}{|c|c|c|c|c|c|c|c|c|c|c|}
\hline \multirow{7}{*}{ YEAB } & \multicolumn{10}{|c|}{$\begin{array}{l}\text { cose of } \\
\text { REMOUAL }\end{array}$} \\
\hline & & & & Coss or & Prom & \multirow{4}{*}{\multicolumn{2}{|c|}{$\begin{array}{l}\text { TOPAL } \\
\text { DISC. AT }\end{array}$}} & \multirow{5}{*}{ 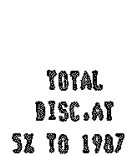 } & \multirow{5}{*}{ 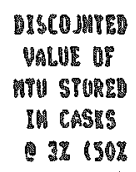 } & \multirow{6}{*}{ 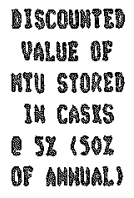 } \\
\hline & & & & LOADINE & 5TOBA6E & & & & & \\
\hline & AHOHMI PUEL & & cost of & \& PLACE- & PRLPARATIOH & & & & & \\
\hline & STORED AR BWTU & WO. of & MPTAL & MEHP IN & rote & & & & & \\
\hline & - & MEIAL & CASTS & STORAGE & SHLPMLW & TOTAL & 38901898 & & & \\
\hline & 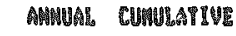 & CASPSS & 800008 & $18000)$ & $(8000)$ & $(8000)$ & $18000)$ & 180001 & 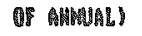 & \\
\hline
\end{tabular}

\begin{tabular}{|c|c|c|c|c|c|c|c|c|c|c|c|}
\hline 1997 & 24 & 54 & 1 & 958 & (c) & & 984 & 964 & 104 & 12 & 12 \\
\hline 1980 & 100 & 160 & 4 & 3,224 & 30 & & 3,854 & 3,742 & 3,071 & 51 & 50 \\
\hline 1979 & 163 & 323 & 6 & 5,223 & 45 & & 5,248 & 4,968 & 4,778 & 77 & 74 \\
\hline 1990 & 126 & 449 & 4 & 3,100 & 30 & & 3,196 & 2,1772 & 2,711 & 58 & 54 \\
\hline 1991 & 238 & 687 & 0 & 8,120 & 60 & & 8,180 & 5,491 & 5,084 & 106 & 98 \\
\hline 1992 & 206 & 973 & 10 & 7,520 & 75 & & 7,595 & 6,552 & 5,951 & 123 & 182 \\
\hline 1993 & 258 & 1831 & 9 & 8,887 & 68 & & 6,755 & 5,657 & 5,040 & 108 & 96 \\
\hline 1994 & 428 & 1859 & 15 & 11,025 & 113 & & 11,130 & 9,058 & 7,015 & 174 & 152 \\
\hline 1995 & 365 & 2024 & 13 & 8,438 & 98 & & 9,536 & 7,528 & 6,454 & 144 & 124 \\
\hline 1998 & 454 & 2770 & 16 & 11,520 & 120 & & 11,810 & $\pi_{1}, 92$ & 7,503 & 174 & 148 \\
\hline 1997 & 516 & 2994 & 18 & 18,834 & 135 & & 12,969 & 1,650 & 7,962 & 192 & 150 \\
\hline 1988 & 169 & 3163 & 6 & 4,254 & 45 & & 4,299 & 3,108 & 2,514 & $\$ 1$ & 49 \\
\hline 1989 & 272 & 3435 & 9 & 6,363 & 68 & & 0,431 & 4,510 & 3,511 & 45 & 76 \\
\hline 2000 & 390 & 3825 & 13 & 8,152 & 98 & & 8,250 & 4,209 & 1,005 & 133 & 103 \\
\hline 2001 & & & (2) & & & 2 & 2 & 2 & 1 & & \\
\hline 2002 & & & $(30)$ & & & 35 & 35 & 22 & 17 & & \\
\hline 2003 & & & (89) & & & 80 & 80 & 50 & 37 & & \\
\hline 2004 & & & (31) & & & 36 & 36 & 22 & 16 & & \\
\hline
\end{tabular}

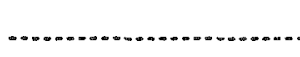

132

\begin{tabular}{|c|c|}
\hline 98,024 & 913 \\
\hline 78,522 & 711 \\
\hline 352.1 & $\$ .5$ \\
\hline 6,340 & 685 \\
\hline 152.3 & 5.5 \\
\hline
\end{tabular}

99,170

70,600

89,105

1,508

THบAL DISC จ3

TUTA MIS อระ UMII COST/RE 


\section{TละLE I-1B}

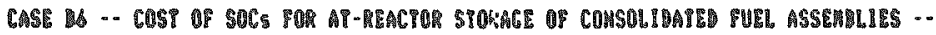

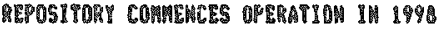

OME - PITE USE OF SOC ?OI SHIPMEMT

$$
\text { CAPACIY - }(32 / 76)
$$

(50) of ar Storage Requipenests Pronded Iy Cashs)

\begin{tabular}{|c|c|c|c|c|c|c|c|c|c|c|}
\hline \multirow{6}{*}{ YEAR } & & \multirow{6}{*}{$\begin{array}{l}\text { MD. Of } \\
\text { METAL }\end{array}$} & \multirow{6}{*}{$\begin{array}{l}\text { COSP OP } \\
\text { METAL } \\
\text { CASISS }\end{array}$} & \multicolumn{3}{|c|}{$\begin{array}{l}\text { COST or } \\
\text { REMOYAL }\end{array}$} & \multirow[b]{4}{*}{ IUYติ } & \multirow{5}{*}{$\begin{array}{c}\text { TOTAL } \\
\text { IISC.AT } \\
\text { 58 TO I9E }\end{array}$} & \multirow{5}{*}{ 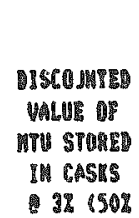 } & \multirow{6}{*}{ 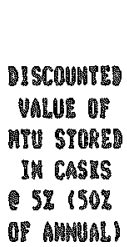 } \\
\hline & & & & cos? of & PRom & & & & & \\
\hline & & & & LOADING & STOEAGE & & & & & \\
\hline & 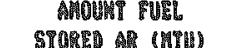 & & & 8 PLACE- & $\begin{array}{l}\text { PREPARATIOA } \\
\text { POR }\end{array}$ & & & & & \\
\hline & ……................ & & & Shat In & & & ISC. 呚 & & & \\
\hline & AพWUAL CUพULATIUE & & & $\begin{array}{c}\text { SPORAGE } \\
180001\end{array}$ & $\begin{array}{l}\text { SHIPrent } \\
\text { (5000) }\end{array}$ & $\begin{array}{l}109 \mathrm{~T} \text { (16000) } \\
10000\end{array}$ & $\begin{array}{c}32901907 \\
18000)\end{array}$ & $\begin{array}{c}58.01907 \\
(0000)\end{array}$ & 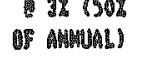 & \\
\hline
\end{tabular}

\begin{tabular}{|c|c|c|c|c|c|c|c|c|c|c|c|}
\hline 1907 & 24 & 54 & 1 & 956 & 7 & & 983 & 963 & 963 & 12 & 12 \\
\hline 1938 & 106 & 160 & 2 & 1,912 & 15 & & 1,927 & 1,87 & 1,835 & 51 & 50 \\
\hline 1989 & 163 & 323 & 3 & 2,868 & 22 & & 2,090 & 2,724 & 2,822 & $n$ & 74 \\
\hline 1990 & 128 & 448 & 3 & 2,700 & 22 & & 2,722 & 2,891 & 2,352 & 58 & 54 \\
\hline 1991 & 238 & 687 & 5 & 3,900 & g7 & & 3,937 & 3,498 & 3,239 & 106 & 8 \\
\hline 1902 & 208 & 973 & 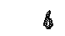 & 4,600 & 45 & & 0,853 & 4,013 & 3,645 & 123 & 112 \\
\hline 1993 & 258 & 1231 & 3 & 3,795 & y & & $3, \otimes 32$ & 3,209 & 2800 & 108 & 80 \\
\hline 1994 & 428 & 1659 & 9 & 8,750 & 67 & & 8,817 & 5,543 & $f_{0}: 145$ & 174 & 152 \\
\hline 1095 & 365 & 2024 & 1 & 5,936 & 60 & & 5,98 & 48733 & 4,058 & 144 & 124 \\
\hline 1996 & 454 & 2478 & 9 & $\$_{s}, 624$ & 67 & & 8,691 & 5,128 & 4,313 & 174 & 146 \\
\hline 1997 & 516 & 2994 & 11 & 8,030 & 82 & & 8,112 & 6,036 & 4,880 & 192 & 158 \\
\hline 1998 & 169 & 3163 & 4 & $\mathbf{2}_{8}, 900$ & 30 & & 2930 & 2,117 & 1,713 & $\$ 1$ & 49 \\
\hline 1999 & 272 & 34วร & 6 & 4,344 & 45 & & 7,380 & 3,078 & 2,344 & 85 & 78 \\
\hline 2000 & 390 & 3825 & 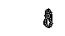 & 5,760 & 60 & & 5,820 & 3,963 & 3,008 & 133 & 103 \\
\hline 2001 & & & (11) & & & 1 & 1 & 1 & 1 & & \\
\hline 2002 & & & (18) & & & 22 & 22 & 14 & 10 & & \\
\hline 2003 & & & (A1) & & & A9 & 48 & 31 & 23 & & \\
\hline 2004 & & & $(19)$ & & & 23 & 23 & if & 10 & & \\
\hline
\end{tabular}

TeTal IISC UHIT COST/MC

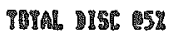

MII COST/R

c...........

\begin{tabular}{|c|c|c|c|c|c|c|c|}
\hline 41,083 & 588 & 89 & 41,77 & 49.428 & 42, & 1,508 & 1,308 \\
\hline $\begin{array}{r}13_{1} 195 \\
132 .\end{array}$ & $\begin{array}{l}473 \\
6.3\end{array}$ & 59 & & $\begin{array}{r}49,420 \\
132.8\end{array}$ & & & \\
\hline $\begin{array}{r}12,545 \\
32.5\end{array}$ & 410 & 4 & & & $\begin{array}{l}42,096 \\
332.1\end{array}$ & & \\
\hline
\end{tabular}


TAILE I-18

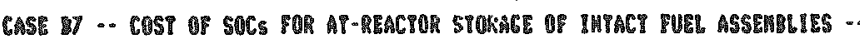

REPOSIYORY COMHEHCES OFERATION IV 1996

SHIPHEMIIIN OUERPACEED SOC

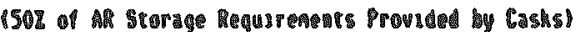

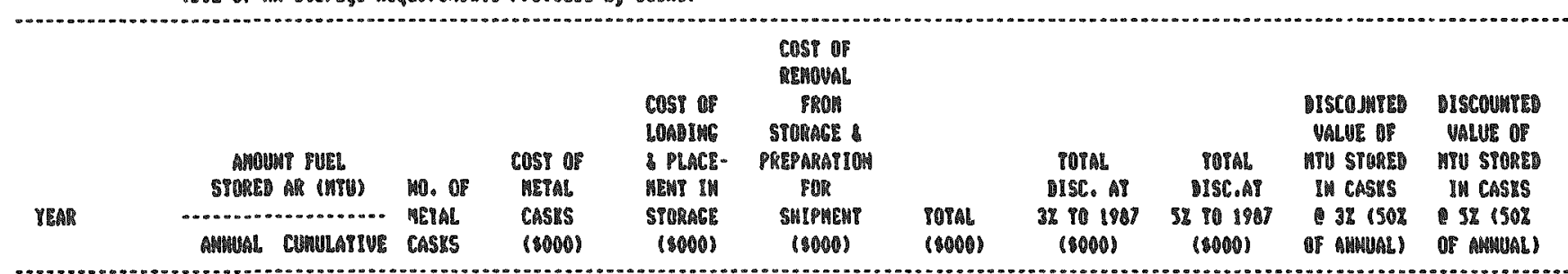

1907
1984
1989
1990
1991
1902
1993
1994
1995
1096
1997
1996
1994
2000
2001
2002
2003
2004
2005
2008
2007
2008
2009
2010
2011
2012
2013
2014
2015
2016
2017
2010
2019
2020

$\begin{array}{rr}24 & 54 \\ 106 & 180 \\ 163 & 323 \\ 128 & 449 \\ 230 & 687 \\ 286 & 973 \\ 258 & 1231 \\ 428 & 1659 \\ 365 & 2024 \\ 454 & 2478 \\ 516 & 2994 \\ 189 & 3183 \\ 272 & 3435 \\ 390 & 3825\end{array}$

1
6
8
8
10
8
15
13
18
18
6
9
13
121
1301
1491
1311

956
3,824
5,223
3,108
8,120
7,520
6,607
11,025
8,438
11,520
12,834
4,254
8,363
8,152

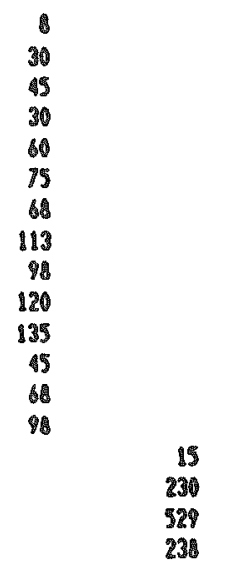

984
3,854
5,268
3,138
6,160
7,585
8,755
11,130
9,530
11,840
12,960
1,290
6,431
9,250
15
230
529
230

964
3,742
4,968
2,072
5,491
8,558
5,657
9,056
7,528
8,921
8,650
3,106
7,510
6,298
10
148
330
184

864
3,871
4,778
2,711
5,804
5,851
5,804
7,915
6,854
7,5003
7,962
2,514
3,561
7,805
8
111
242
104

$\begin{array}{rr}12 & 12 \\ 51 & 50 \\ 77 & 74 \\ 51 & 54 \\ 106 & 98 \\ 123 & 112 \\ 108 & 86 \\ 174 & 152 \\ 144 & 124 \\ 174 & 196 \\ 192 & 158 \\ 81 & 49 \\ 95 & 76 \\ 133 & 103\end{array}$

TRTAL

TUTAL DISC BS:

แพII COST/MG

TBPAL DISC TSE uars tosim/

\begin{tabular}{|c|c|c|c|c|c|c|c|}
\hline $132 \quad 98,024$ & 993 & 1,012 & $100_{p} 030$ & 70,9185 & 69,499 & 1,508 & 1,308 \\
\hline 76.522 & 791 & 631 & & 79,945 & & & \\
\hline 852.1 & 0.5 & $\$ .1$ & & 853.0 & & & \\
\hline 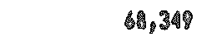 & 885 & 485 & & & 69,840 & & \\
\hline 852.3 & 8.8 & 0.4 & & & 953.2 & & \\
\hline
\end{tabular}


IAMLI $\$-20$

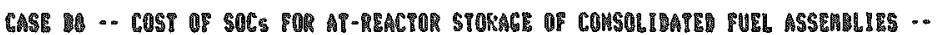

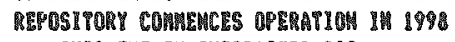

$$
\begin{aligned}
& \text { SAIPAEAP IIM OVERPACIE SOC } \\
& \text { CAPACITY - (32/76) }
\end{aligned}
$$

1502 of a storage hequzrenents Provided by Cashs

\begin{tabular}{|c|c|c|c|c|c|c|c|c|c|c|c|}
\hline YraA & $\begin{array}{r}\text { AMOLM } \\
\text { STORED } \\
\text { AMULAL }\end{array}$ & 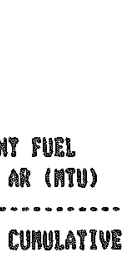 & $\begin{array}{l}\text { MD. Of } \\
\text { METAL } \\
\text { CASIS }\end{array}$ & $\begin{array}{l}\text { COST OS } \\
\text { METAL } \\
\text { CASES } \\
\text { (POOO) }\end{array}$ & $\begin{array}{l}\text { COST OP } \\
\text { LOABINC } \\
\text { PLACE- } \\
\text { MEMT IM } \\
\text { SPORACE } \\
\text { (\$OOO) }\end{array}$ & 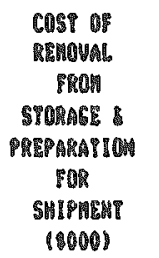 & $\begin{array}{l}\text { IOPAL } \\
\text { (\$ODO) }\end{array}$ & $\begin{array}{l}\text { PDYA } \\
\text { IISC. } \\
3 \% \text { T0 } 1987 \\
\text { (8000) }\end{array}$ & 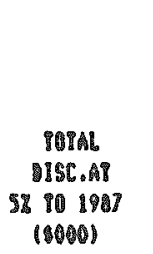 & 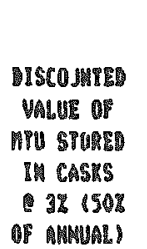 & 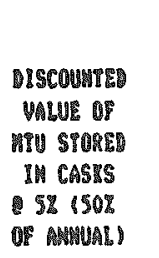 \\
\hline 1908 & 24 & 54 & $\mathbb{1}$ & 758 & 8 & & 963 & 963 & 963 & 12 & 12 \\
\hline 198 & 106 & 180 & 2 & 1,912 & 15 & & 1,827 & 1,871 & 1,835 & 51 & 50 \\
\hline 1989 & 163 & 323 & 3 & 2,868 & 22 & & 2,890 & 2,724 & 2,622 & 77 & 74 \\
\hline 1990 & 120 & 949 & 3 & 2,700 & 22 & & 2,122 & 2,491 & 2,352 & 58 & 54 \\
\hline 1991 & 230 & 687 & 5 & 3,900 & 37 & & 3,938 & 3,198 & 3,239 & 108 & 98 \\
\hline 1982 & 24 & 973 & 8 & 9,608 & 45 & & 4,853 & 4,013 & 3,645 & 123 & 112 \\
\hline 1993 & 258 & 1231 & 5 & 3,795 & 37 & & 3,832 & 3,209 & 2,880 & 100 & 96 \\
\hline 1994 & 228 & 1859 & $\theta$ & 6,750 & \$7 & & 6,817 & 5,543 & 4,845 & 174 & 152 \\
\hline 1995 & 365 & 2024 & 1 & 5,938 & so & & 5,996 & 4,733 & 9058 & 194 & 124 \\
\hline 1998 & 454 & 2478 & 8 & 8,624 & 87 & & 6,891 & 5,126 & 4313 & 174 & 198 \\
\hline 1997 & $\$ 16$ & 2994 & 11 & 0,030 & 22 & & 0,112 & 0,036 & 4,980 & 192 & 158 \\
\hline 1998 & 169 & 3163 & 1 & 2,900 & 30 & & 2,930 & 2,117 & 1,113 & $\$ 1$ & 49 \\
\hline 1999 & 272 & 3435 & 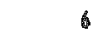 & 4,394 & 45 & & 4,380 & 3,07 & 2,844 & 95 & 76 \\
\hline 2000 & 390 & 3825 & 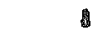 & 5,760 & 80 & & 5,820 & 3,983 & 3,008 & 133 & 103 \\
\hline 2001 & & & (1) & & & 8 & 8 & 5 & 4 & & \\
\hline 2002 & & & (18) & & & 138 & 138 & 9 & 87 & & \\
\hline 2003 & & & (41) & & & 315 & 315 & 196 & 144 & & \\
\hline 2004 & & & $(19)$ & & & 148 & 148 & 8 & 84 & & \\
\hline 2005 & & & & & & & & & & & \\
\hline 2008 & & & & & & & & & & & \\
\hline 2007 & & & & & & & & & & & \\
\hline 2008 & & & & & & & & & & & \\
\hline 2008 & & & & & & & & & & & \\
\hline 2010 & & & & & & & & & & & \\
\hline 2011 & & & & & & & & & & & \\
\hline 2012 & & & & & & & & & & & \\
\hline 2013 & & & & & & & & & & & \\
\hline 2014 & & & & & & & & & & & \\
\hline 2015 & & & & & & & & & & & \\
\hline 2016 & & & & & & & & & & & \\
\hline 2017 & & & & & & & & & & & \\
\hline 2018 & & & & & & & & & & & \\
\hline 2018 & & & & & & & & & & & \\
\hline 2020 & & & & & & & & & & & \\
\hline TOPAL & & & 80 & 61,093 & 596 & 607 & $\$ 2,2 B S$ & 49,747 & 43,234 & 1,500 & 18306 \\
\hline TOPAL MISC E3Z & & & & 48,895 & 473 & 379 & & 49,747 & & & \\
\hline unIP cost/ng & & & & $\$ 32.4$ & 1.3 & 8.3 & & 333.0 & & & \\
\hline TEบนL МISC อระ & & & & 42,545 & 410 & 279 & & & 43,234 & & \\
\hline HMI\% cosinte & & & & 332.4 & 8.3 & $\$ .2$ & & & 933.1 & & \\
\hline
\end{tabular}


TABLE $I-21$

SENSITIVITY OF COSTS OF USE OF TSCS OR SOCS IN AT-REACTOR STORAGE OF INTACT FUEL ASSEMBLIES TO INCREASES IN CASK CAPACITY

$(\$ / \mathrm{kgU}, 1987)^{\mathrm{b}}$

Unit Costs For Casks of Indicated Capacity

\begin{tabular}{|c|c|c|c|c|}
\hline $\begin{array}{c}\text { Base Case } \\
21 \text { PWR/46 BWR } \\
(9.26 \text { MTU Capacity) } \\
\end{array}$ & $\begin{array}{c}\text { Case A } \\
26 \text { PWR/52 BWR } \\
\text { (11.16 MTU Capacity) }\end{array}$ & $\begin{array}{r}32 \\
(14.47 \\
\end{array}$ & & $\begin{array}{l}\text { se B } \\
176 \text { BWR } \\
\text { U Capacity) } \\
\end{array}$ \\
\hline$\$ 88.7$ & $\$ 74.3$ & & $\$$ & 58.3 \\
\hline 79.6 & 66.6 & & & 52.7 \\
\hline 80.1 & 67.0 & & & 53.0 \\
\hline 81.6 & 68.5 & & & 54.5 \\
\hline 55.0 & 55.0 & & & 55.0 \\
\hline
\end{tabular}

\footnotetext{
Assumed On-Time (1998) Repository

b Averaged at a $3 \% /$ year discount rate

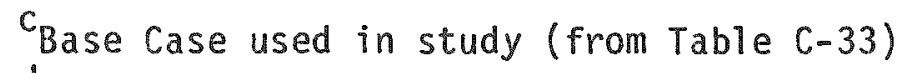

${ }^{d}$ For comparison with TSC and SOC costs (from Table C-33)
} 
TABLE I-22

SENSITIVITY OF COSTS OF USE OF TSCS OR SOCS IN AT-REACTOR STORAGE OF CONSOLIDATED FUEL CANS TO INCREASES IN CASK CAPACITY

$(\$ / \mathrm{kgU}, 1987)^{\mathrm{b}}$

Unit Costs For Casks of Indicated Capacity

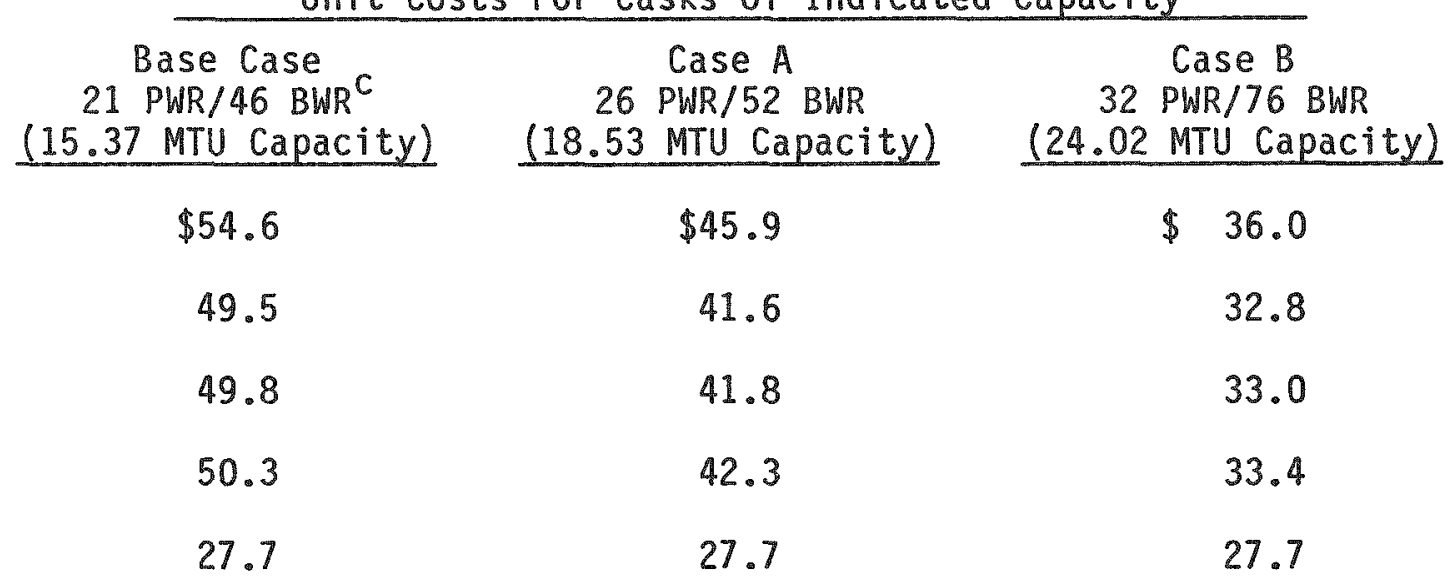

\footnotetext{
assumed On-Time (1998) Repository

b Averaged at a $3 \% /$ year discount rate

$C_{\text {Base }}$ Case used in study (from Table C-34)

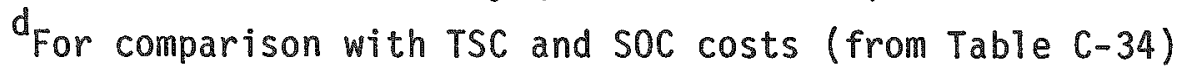




\subsubsection{Discussion of Cost Comparisons}

1.1.3.1 Comparison of Costs for At-Reactor Storage as Intact Fuel Assemblies

From Table I-21 it can be seen that the least costly option compared with the Case A higher capacity metal cask is the concrete cask. The concrete cask option is more than $\$ 11 / \mathrm{kgl}$ less expensive than the least cost7y SOC option. Of the three SOC options, the least costly is the one-time, one-way shipment of the SOC to a DOE facility. The next least costly SOC option is shipping the SOC in an overpack to a DOE facility. The most costly SOC option is the storage-only option, where a DOE-supplied transport cask is required to ship the spent fuel stored in the SOC to a DOE facility. The difference in cost between the most costly and least costly SOC options is small, less than $\$ 2 / \mathrm{kgU}$. For Case $A$, the TSC option is the most costly option and is $\$ 6-8 / \mathrm{kgU}$ more than the SOC options.

For the Case B higher capacity metal casks, the SOC options are less costly than the concrete cask option. However, the TSC option remained more costly than the concrete cask option. It should be noted that the SOC options are only marginally less costly than the concrete cask option. There is also greater uncertainty in the cost estimated for the concrete casks. Because of this uncertainty, no conclusions should be drawn beyond the statement that atreactor costs for high capacity TSCS and SOCs appear to be similar in magnitude to the costs of concrete casks.

\subsubsection{Comparison of Costs for At-Reactor Storage as Consolidated Fuel}

From the summary table for cases with consolidated fuel (Table I22) it can be seen that the concrete cask is the least costly option compared with both Case $A$ and Case $B$ higher capacity metal casks. The concrete cask option is almost $\$ 14 / \mathrm{kgU}$ less than the least costly SOC option for Case A (26/52) capacity metal casks. The concrete cask option is $\$ 5 / \mathrm{kgU}$ less than the least costly SOC option for Case B (32/76) capacity metal casks. These results differ from the results for intact fuel assembly cases due to the additional cost for canning intact fuel to be stored in concrete casks. The cost penalty for canning intact fuel does not extend to the consolidated fuel cases. The costs for canning consolidated fuel were excluded from all consolidated fuel cases because it was assumed that consolidated fuel had 
already been canned prior to storage. Therefore, the costs for the concrete cask options for consolidated fuel are significantly lower than for intact fuel due to the exclusion of canning costs.

Like the cases for intact fuel, of the three SOC options for consolidated fuel; the least costly is the one-time, one-way shipment of the SOC to a DOE facility. The next least costry SOC option is shipping the SOC in an overpack to a DOE facility. The most costly SOC option is again the storage-only option. In all (32/76) cases, the TSC option is the most costly option.

1.2 SENSITIVITY OF SAVINGS WITHIN THE DOE SYSTEM TO CHANGES IN THE CAPACITY OF METAL STORAGE CASKS

The impact of increases in the capacity of metal storage casks on savings realized in the DOE system from the use of such higher capacity casks was determined. Impacts were determined for the transport of spent fuel from reactors to DOE facilities, replacement of lag storage capacity, replacement of MRS modules, and transport from MRS to repository facilities. These impacts are described in the following sections.

1.2.1 Sensitivity of Savings in the Cost of Transport from Reactors to DOE Facilities to Changes in Cask Capacity

The life cycle and resulting unit costs for the transport of spent fuel from a reactor to a DOE facility were calculated for cases where a TSC or SOC is used to replace a DOE transport cask in the transport fleet. For comparison purposes, the TSC or SOC and the DOE transport cask were all assumed to have the same increased capacities. It was also assumed that the total number of shipments/year over a given distance remained constant.

It was determined that the unit costs for transport decreased for the higher capacity casks whether they were DOE transport casks, TSCS or SOCs, because of the higher payloads involved. Table I-23 shows the results of the unit cost determinations. The results shown in the table demonstrate that there is a small cost savings associated with the use of a TSC over the DOE transport cask (well less than $\$ 1 / \mathrm{kgU}$ ) in every case. This is because the cost of the TSC is not included in the total capital cost for that option. It is assumed that the utility paid for the TSC and, therefore, the TSC costs are 
TABLE I-23

SENSITIVITY OF SAVINGS IN THE COST OF TRANSPORT FROM REACTORS TO DOE FACILITIES RESULTING FROM THE USE OF TSCS FOR TRANSPORT TO INCREASES IN CASK CAPACITY

(1987 Dollars)

\section{Cask Scenario}

Base Case (Cask Capacity: 21 PWR or 46 BWR assembilies)

- DOE-Supplied Transport Cask

- TSC Used in Repetitive Shipments: DOE Avoids Purchase of Transport Cask

$\stackrel{\mathfrak{s}}{\stackrel{0}{6}}$

\begin{tabular}{ccc}
$\begin{array}{c}\text { Unit Cost }(\$ / \mathrm{kgU})^{b} \text { for } \\
\text { Transport for Indicated Distances } c, d\end{array}$ \\
\hline 300 Miles & 900 Miles & 2300 Miles
\end{tabular}

$\$ 3.91$

$3.60(0.31)$

$2.99(0.26)$

$2.30(0.20)$
$\$ 6.83$

$6.36(0.47)$

$\$ 10.61$

$10.04(0.57)$

5.67

8.80

$5.28(0.39)$

$8.33(0.47)$ Cask

Case B (Cask Capacity: 32 PWR or 76 BWR assemblies)

- DOE-Supplied Transport Cask

- TSC Used in Repetitive Shipments: DOE Avoids Purchase of Transport Cask

\footnotetext{
antact fuel assemblies

b Averaged at a $3 \% /$ year net discount rate

G One-way distances shown; costs are for round trip

d Figures in parentheses are cost savings resulting from the use of utility-furnished TSC or SOC for transport instead of DOE-supplied transport cask
} 
accounted for in total at-reactor costs. However, the cost of the DOE transport cask is included in the capital costs for that option. The cost savings of the TSC over the DOE-transport cask is small because the capital costs are only a small portion of the total life cycle transport costs. The majority of the life cycle transport costs consist of annual operating costs, freight charges, and escort charges which are the same for the TSC and DOE transport casks.

\subsubsection{Sensitivity of Savings from Replacement of Lag Storage Capacity to Changes in Cask Capacity}

The estimated savings to the DOE system resulting from the reduction in the amount of lag storage required at either the Integral MRS or repository facility is $\$ 233 / \mathrm{kgU}$ (assuming all lag storage capacity is in-cell storage), as described in Appendix E. It is recognized that if DOE determines the use of metal storage casks for lag storage is an acceptable alternative to in-cell storage, DOE could purchase SOCs for lag storage at a cost of about $\$ 735$-thousand (1987 dollars), the estimated cost of the 50 th unit. Therefore, the savings to the DOE system for the lag storage capacity provided by a TSC or SOC would be equivalent to the cost of a SOC. On a unit cost basis, the savings within the DOE system would go down as the capacity of the delivered TSCS or SOCs increases as shown in Table I-24. However, the savings on a per cask basis does not change with increasing capacity. 
TABLE I-24

SENSITIUITY OF SAVINGS FROM REPLACEMENT OF IN-CELL LAG STORAGE CAPACITY WITH TSCS OR SOCS TO INCREASES IN CASK CAPACITY

TSC or Soc Capacity

Base Cask (Cask Capacity: 21 PUR or 46 BUR assenblies or cans)

T. Cask A (Cask Capacity: 26 PWR or $\omega$ 52 BWR assemblies or cans)

Case (Cask Capacity: 32 PWR or 76 BWR assemblies or cans)
At MRS or Repository With No MRS In System Maximum No. Casks That Can Be Used For

Lag Storage

$54 \quad(32)$

45 (27)

35 (21) Intact Fuel Fuel

$\$ 79.4$

$\$ 47.8$

39.7

30.6

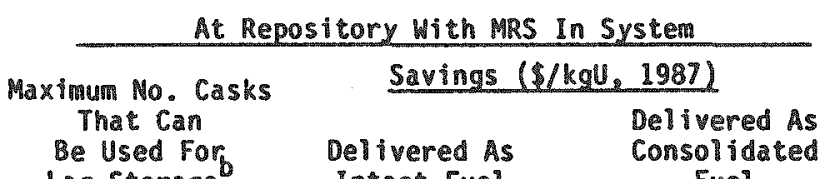

Delivered As Consolidated
Lag storage

Intact Fuel

$$
\begin{array}{rrr}
\$ 75.9 \text { (PHR) } & \$ 45.6 & \text { (PHR) } \\
56.7 \text { (BUR) } & 34.0 & \text { (BUR) }
\end{array}
$$

21

61.3 (PUR)

50.1 (BWR)

36.8 (PWR)

30.1 (BWR)

17
49.8 (PWR)
34.3 (BWR) $\begin{array}{ll}29.9 & \text { (PUR) } \\ 20.6 \text { (BUR) }\end{array}$

Numbers of casks that can be used to provide 500 MTU lag storage of intact spent fuel. Numbers in parentheses are numbers of casks that can be used to provide 500 MTU lag storage of consolidated fuel.

Assumes cans of fuel rods are stored, $0.92 / \mathrm{can}$. See note a. 
1.2.3 Sensitivity of Savings from Replacement of MRS Modules to Changes in Cask Capacity

Savings can be realized by substituting TSCS or SOCs for MRS storage modules at the MRS facility. The savings involved is equivalent to the cost of the storage capacity replaced by the TSCS or SOCS. The savings involved in the use of the higher capacity casks was determined; a summary of the results is set forth in Table I-25. The savings for TSCS or SOCs delivered containing BWR spent fuel is less than TSCS or SOCs delivered containing PWR spent fuel in every case because of the added expense to replace the BWR fuel basket before the TSC or SOC can be used as a replacement for a MRS storage module. The results of the calculations show that there is no increase in savings (on a $\$ / \mathrm{kgU}$ basis) for higher capacity TSCs or SOCs delivered containing PWR spent fuel. However, there is a significant increase in savings or, more appropriately, a significant decrease in the cost penalty, for higher capacity TSCS or SOCs delivered containing BWR spent fuel. The increase in savings for higher capacity TSCS or SOCs delivered containing BWR spent fuel is because the replacement of the basket is assumed to be a fixed cost per cask that is not dependent on cask capacity. The unit cost is determined by dividing the cost per cask by the cask capacity (in kgu). Therefore, when the capacity of the cask is increased and the cost per cask for replacement of the BWR basket remains unchanged, the cost penalty (in $\$ / \mathrm{kgU}$ ) goes down and the savings in the DOE system increases proportionately.

It should be noted that fewer high capacity casks would be required for whatever portion of the MRS storage capacity that is met through the use of TSCS or SOCS. 


\section{TSC Capacity}

Base Case (Cask Capacity: 21 PWR or 46 BWR assemblies or cans)

$\stackrel{\leftrightarrow}{\omega}$

Case A (Cask Capacity: 26 PWR or 52 BWR assemblies or cans)

Case B (Cask Capacity: 32 PWR or 76 BWR assemblies or cans)

Averaged at a $3 \% /$ year discount rate.
Maximum Number TSCS or SOCS Accepted At MRS

240

194

158
Savings $(\$ / \mathrm{kgU}, 1987)^{2}$

Delivered As

Intact Fuel

35.5 (PWR)

10.1 (BWR)

35.5 (PWR)

17.5 (BWR)

35.5 (PWR)

19.1 (BWR)
21.4 (PWR)

6.1 (BWR)

21.4 (PWR)

10.6 (BWR)

Delivered As Consolidated Fuel

21.4 (PWR) 11.5 (BWR) 
1.2.4 Sensitivity of the Cost of Transport from the MRS to a Repository to Changes in Cask Capacity

The impact of using higher capacity TSCs for the shipment of cans of consolidated spent fuel from a MRS facility to a repository on the cost of such shipments was estimated. It was expected that the higher capacity TSCS would be more efficient at this task than the base case TSC which has a 21 MRS can capacity. The life cycle transport costs were determined for TSCs used for repetitive shipments of MRS cans of consolidated fuel and having cask capacities for 26 MRS cans and 32 MRS cans. The transport cost for these higher capacity TSC cases were compared with the costs for a standard cask. Table I-26 provides a summary of the unit costs for transport from the MRS to three repository locations in TSCS of different capacities and in a standard transport cask. The unit costs are higher for the Case A (26 PWR/52 BWR assembly) capacity TSC than for the standard cask, thus, no transport cost savings can be realized by using this capacity TSC in place of a standard cask. For the Case B (32 PWR/76 BWR assembTy) capacity TSC, the unit transport costs are about the same for the TSC and standard cask. 
TABLE I-26

SENSITIVITY OF COSTS OF TRANSPORT FROM MRS TO REPOSITORY FACILITY

TO INCREASES IN TSC CAPACITY

\section{Destination}

To Salt Repository in Cans

To Basalt Repository in Cans

To Tuff Repository in Cans

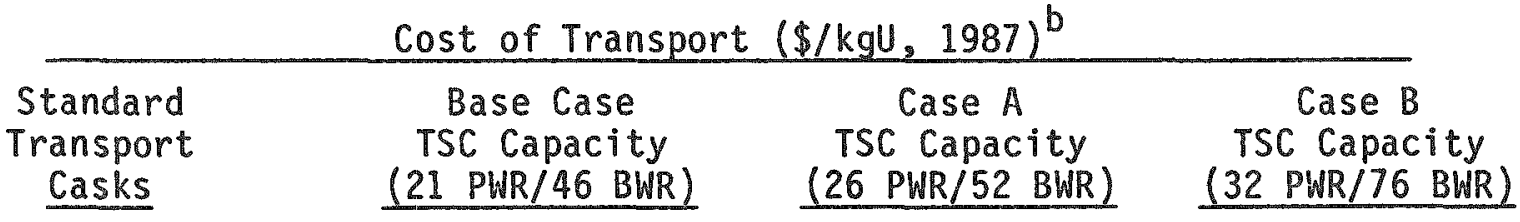

$\$ 4.12$

$\$ 5.14$

$\$ 4.49$

$\$ 4.01$

6.53

8.16

7.14

6.38

6.12

7.71

6.77

6.02

assumes TSC is used repetitively (i.e., that it is made part of the transport cask fleet and used to make repeated round trip shipments).

${ }^{b}$ Averaged at a $3 \% /$ year discount rate 
The impact of changes in the cost for fabrication of metal storage casks on the cost of using the casks at the reactor site, and on the savings to the DOE system as a result of a utility delivering spent fuel to DOE in such casks, was determined. Two variants in the cost of TSCs were considered; TSCS that cost nominally $\$ 400$-thousand more than the base case TSC, and TSCS that cost nominally $\$ 400$-thousand less. This variation was applied to the cost of the initial cask procured for the base case TSC ( $\$ 990$-thousand) oe SOC ( $\$ 880$ thousand), as applicable. Variations in the cost of SOCs was not calculated separately, but the absolute changes caused by cost variations to TSCS would be applicable to SOCs as well. In calculating the impact of cask cost changes on at-reactor costs and costs in the DOE system, the same methodologies were used as are described in Appendices $A, B, C, D, E, F$, and $G$ for the base case.

\subsection{SENSITIVITY OF AT-REACTOR COSTS TO CHANGES IN THE COST FOR METAL STORAGE CASKS}

2.1.1 Sensitivity of At-Reactor Operations Costs to Changes in Cask Cost

A change in the cost of TSCS or SOCS will have no impact on the atreactor operations costs, since cask cost is not included in the cost of operation at the reactor site.

\subsubsection{Sensitivity of At-Reactor Life Cycle and Unit Costs to Changes in Cask Cost}

An analysis was performed of the impact of changes in the costs for metal storage casks on the life cycle and resulting unit costs involved with the use of the casks at the reactor site. The analysis was performed using the same methodology as was used for determining the at-reactor cask costs for the base case (as described in Appendix C). Two variants in the cost of TSCS were considered, one in which the cask cost $\$ 580$-thousand and one in which the cask cost $\$ 1,380$-thousand. The results of the analysis is set forth in Table I-27. From the results shown in the table, it can be seen that a $\$ 100-$ thousand change in cask cost causes a $\$ 8-9 / \mathrm{kgu}$ variation in at-reactor costs. 


\section{TO CHANGES IN CASK COST}

$(\$ / \mathrm{kgu}, 1987)^{\mathrm{a}}$

\section{On-Time Repository}

TSC Shipped Directly to DOE

(1st Cask Cost $\$ 580$-thousand)

TSC Shipped Directly to DOE

(Base Case -- Ist Cask Cost $\$ 990$-thousand)

TSC Shipoed Directly to DOE

(1st Cask Cost $\$ 1,380-$ thousand)

\section{5-Year Repository Delay}

TSC Shipped Directly to DOE

(1st Cask Cost $\$ 580$-thousand)

TSC Shipped Directily to DOE

(Base Case -- Ist Cask Cost $\$ 990$-thousand)

TSC Shipped Directiy to DOE

(Ist Cask Cost \$1,380-thousand)

\begin{tabular}{ccc} 
Loading & $\begin{array}{c}\text { Removal } \\
\text { Loading }\end{array}$ & $\begin{array}{c}\text { From Storage } \\
\text { Elacement }\end{array}$ \\
Equipment Preparation \\
\hline
\end{tabular}

Average Change In Unit Cost Per

$\$ 100 \mathrm{~K}$ Change In

Casks

51.5

$+$

$\$ 0.7$

87.9

0.7

$\$ 0.1$

0.1

122.5

0.7

0.1

Cask cost

10-Vear Repository Delay

TSC Shipped Directly to DOE

(1st Cask Cost $\$ 580$-thousand)

TSC Shipped Directly to DOE

(Base Case - 1st Cask Cost \$990-thousand)

49.7

0.7

0.1

84.5

0.7

$-.1$

118.2

Total

TSC Shipped Directly to DOE

(1st Cask Cost $\$ 1,380$-thousand)

\begin{tabular}{|c|c|c|c|c|}
\hline 48.5 & - & 0.7 & 0.1 & 49.3 \\
\hline 82.8 & - & 0.7 & 0.1 & 83.4 \\
\hline 115.4 & - & 0.7 & 0.1 & 116.2 \\
\hline
\end{tabular}

averaged at a 3\%/year discount rate 
The change in savings within the DOE system which result from decreases and increases in the TSC cost was determined. Impacts on savings were determined for the transport of spent fuel from reactors to DOE facilities, replacement of lag storage capacity, replacement of MRS modules, and transport from MRS to repository facilities. These impacts are described in the following sections.

\subsubsection{Sensitivity of Savings in the cost of Transport from Reactors to DOE Facilities to Changes in Cask Cost}

The impact on savings in the cost of transport between reactors and DOE facilities as a result of changes in the cost of metal storage casks and DOE transport casks were calculated. Changes in the cost of TSCS or SOCs did not impact the savings per se, since the cost of the cask was not included in the calculations. However, it was reasoned that if the cost of a TSC were to vary, the cost of a DOE transport cask would similarly vary. Therefore, as the cost of a DOE transport cask increased or decreased, the cost of transporting spent fuel in the cask would correspondingly increase or decrease -- while the cost of shipping in TSCS or SOCS would remain unchanged from the base case.

Life cycle costs for transport and the resulting unit costs therefor were calculated for two variants in the cost of the DOE transport cask, one in which the cask cost \$580-thousand, and one in which the cask cost $\$ 1,380$-thousand (excluding development costs and the cost of auxiliary equipment). The results of the unit costs obtained for transport in the DOE transport cask and a TSC for shipments having one-way distances of 300 miles, 900 miles and 2,300 miles are summarized in Table I-28. The impact on savings as a result of the use of SOCs would be the same as that shown for TSCS in the table. 
(1987 Dollars)

\section{Cask Scenario}

1st Cask Cost $\$ 990$-thousand ${ }^{e}$

- DOE-Supplied Transport Cask

- TSC Used in Repetitive Shipments: DOE Avoids Purchase of Transport Cask

1st Cask Cost $\$ 580$-thousand ${ }^{e}$

- DOE-Supplied Transport Cask

- TSC Used in Repetitive Shipments: DOE Avoids Purchase of Transport Cask

Ist Cask Cost $\$ 1,380$-thousand ${ }^{e}$

- DOE-Supplied Transport Cask

- TSC Used in Repetitive Shipments; DOE Avoids Purchase of Transport Cask

antact fuel assemblies

b Averaged at a $3 \% /$ year net discount rate

c One-way distances shown

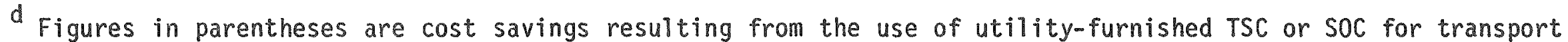
instead of DOE-supplied transport cask

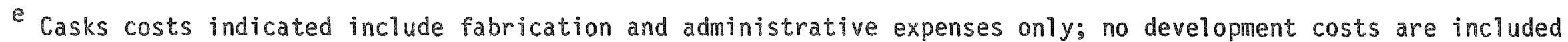
in these figures but such were used in the determination of the unit costs shown in the table.

300 Miles 200 Miles 2300 Miles

$\$ 3.91$

$3.60(0.31)$

3.78

$3.60(0.18)$

4.04

$3.60(0.44)$
7.02

$6.36(0.66)$

6.64

$6.36(0.28)$
10.83

$10.04(0.79)$

$\$ 10.61$

$10.04(0.57)$

10.38

$10.04(0.34)$

(10.04 $(0.79)$

Unit cost $(\$ / \mathrm{kgu})^{\mathrm{b}}$ for Transport for Indicated Distances $c, d$ 
From the results shown in the table it can be seen that a $\$ 100$-thousand change in the cost of DOE transport cask, there is a change in the savings resulting from the use of a TSC or SOC of $\$ 0.033 / \mathrm{kgU}$ for a 300 mile shipment, $\$ 0.048 / \mathrm{kgU}$ for a 900-mile shipment, and $\$ 0.056 / \mathrm{kgU}$ for a 2,300 -mile shipment.

\subsubsection{Sensitivity of Savings from Replacement of Lag Storage Capacity to Changes in Cask Cost}

The savings in the DOE system from using a TSC or SOC for lag storage was determined to be equivalent to the cost of a TSC or SOC. Due to the significantly higher cost of in-cell storage it was felt that DOE would select an alternate means of providing the majority of lag storage capacity in the form of modules. Therefore, DOE could not be expected to pay more than the cost of a TSC or SOC for that storage.

If the TSC or SOC cost changes, the savings in the DOE system changes by an equivalent amount. In other words, if the cost of a TSC or SOC changes and the TSC or SOC remains the least expensive option for providing lag storage, then the savings that DOE would realize by receiving a TSC or SOC from a utility would be equivalent to the new cost of the TSC or SOC.

\subsubsection{Sensitivity of Savings from Replacement of MRS Modules to Changes in Cask Cost}

Changes in the TSC or SOC cask cost have no impact on the savings from substitution of TSCS or SOCS for MRS modules. The savings to the DOE system is determined by the cost of the MRS modules and the capacity of the TSCS or SOCS received into the DOE system. Neither the cost of the MRS modules nor the capacity of the TSC or SOC were assumed to change from the base case, therefore, no change in the amount of savings occurs.

\subsubsection{Sensitivity of the Cost of Transport from the MRS to a Repository to Changes in Cask Cost}

The capital costs used in the life cycle transport calculation for shipment of fuel from the MRS to a repository do not include the cost of the TSC itself. Only the costs for design and licensing and auxiliary equipment are included. It is assumed that the TSC is provided to DOE by a utility that has incurred the cost of the cask. Therefore, an increase in the TSC cost 
would have no impact on the life cycle transport costs from a MRS to a repository. (Note: It is assumed that the cost for a DOE standard cask for shipment from MRS to a repository does not change.)

3.0 SUMMARY OF SENSITIVITY ANAL YSES

3.1 SENSITIVITY OF COSTS AT REACTOR SITE

The changes in costs in the utility spent fuel management system resulting from changes in the capacity and costs of the TSCS or SOCs used for spent fuel storage are summarized in Tables I-29 and I-30, respectively.

The costs shown were derived from the cost information developed in Appendices A, B, and C .- and Sections 1.1 and 2.1 of this Appendix I, plus calculations made using the cost information set forth therein. 
TABLE I-29

SUMMARY OF SENSITIVITY OF COSTS OF USE OF TSCS FOR AT REACTOR STORAGE TO CHANGES IN TSC CAPACITY AND COST

(1987 Dollars)

Unit Costs $(\$ / \mathrm{kg})$ for Indicated Cask Capacities

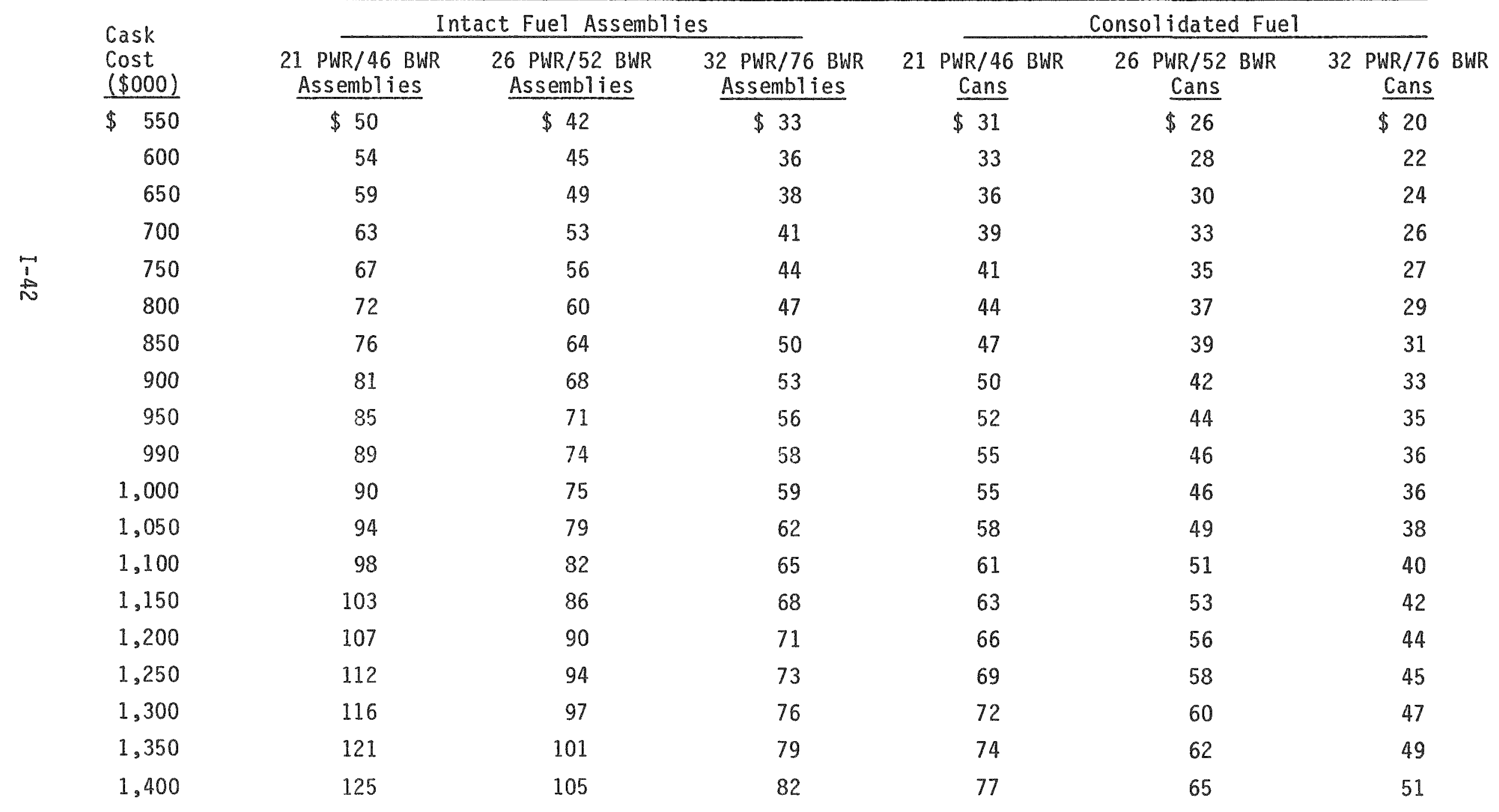


TABLE I-30

SUMMARY OF SENSITIVITY OF COSTS OF USE OF SOCS FOR AT REACTOR STORAGE TO CHANGES IN SOC CAPACITY AND COST (1987 Dollars)

Unit Costs $(\$ / \mathrm{kgU})$ for Indicated Cask Capacities

\begin{tabular}{|c|c|c|c|c|c|c|c|}
\hline \multirow{2}{*}{$\begin{array}{l}\text { Cask } \\
\text { Cost } \\
(\$ 000) \\
\end{array}$} & \multicolumn{3}{|c|}{ Intact Fuel Assemblies } & \multicolumn{3}{|c|}{ Consolidated Fuel } & \\
\hline & $\begin{array}{l}21 \text { PWR/46 BWR } \\
\text { Assemblies }\end{array}$ & $\begin{array}{c}26 \text { PWR/52 BWR } \\
\text { Assemblies }\end{array}$ & $\begin{array}{c}32 \text { PWR/76 BWR } \\
\text { Assemblies }\end{array}$ & $\begin{array}{c}21 \text { PWR/46 BWR } \\
\text { Cans }\end{array}$ & $\begin{array}{c}26 \text { PWR/52 BWR } \\
\text { Cans }\end{array}$ & $\begin{array}{r}32 \text { PWR/76 } \\
\text { Cans }\end{array}$ & BWR \\
\hline$\$ 550$ & $\$ 50$ & $\$ 42$ & $\$ 33$ & $\$ 31$ & $\$ 26$ & $\$ 21$ & \\
\hline 600 & 55 & 46 & 36 & 34 & 29 & 22 & \\
\hline 650 & 59 & 49 & 39 & 37 & 31 & 24 & \\
\hline 700 & 63 & 53 & 42 & 39 & 33 & 26 & \\
\hline 750 & 68 & 57 & 45 & 42 & 36 & 28 & \\
\hline 800 & 72 & 61 & 48 & 45 & 38 & 30 & \\
\hline 850 & 77 & 64 & 51 & 48 & 40 & 32 & \\
\hline 880 & 80 & 67 & 53 & 50 & 42 & 33 & \\
\hline 900 & 81 & 68 & 54 & 51 & 43 & 34 & \\
\hline 950 & 86 & 72 & 57 & 53 & 45 & 35 & \\
\hline 1,000 & 90 & 76 & 60 & 56 & 47 & 37 & \\
\hline 1,050 & 95 & 79 & 63 & 59 & 50 & 39 & \\
\hline 1,100 & 99 & 83 & 66 & 62 & 52 & 41 & \\
\hline 1,150 & 104 & 87 & 69 & 65 & 54 & 43 & \\
\hline 1,200 & 108 & 91 & 72 & 67 & 57 & 45 & \\
\hline 1,250 & 113 & 94 & 75 & 70 & 59 & 46 & \\
\hline 1,300 & 117 & 98 & 78 & 73 & 61 & 48 & \\
\hline 1,350 & 122 & 102 & 81 & 76 & 64 & 50 & \\
\hline 1,400 & 126 & 106 & 83 & 78 & 66 & 52 & \\
\hline
\end{tabular}




\subsection{SENSITIVITY OF SAVINGS IN THE DOE SYSTEM}

The sensitivity of savings in the DOE spent fuel management system to changes in the capacity of the TSCS or SOCs received are summarized in Table I-31. The costs shown were derived from the cost information developed in Appendices D, E, F and $G$-- and Sections 1.2 and 2.2 of this Appendix I, plus calculations made using the cost information set forth therein.

TABLE I-31

SENSITIVITY OF SAVINGS REALIZED IN DOE SYSTEM AS A RESULT OF RECEIVING TSCS OR SOCS FROM UTILITIES CONTAINING INTACT FUEL ASSEMBLIES TO INCREASES IN CASK CAPACITY ${ }^{2}$ $(\$ / \mathrm{kgU}, 1987)^{\mathrm{b}}$

\begin{tabular}{l} 
Avoided Costs Resulting From \\
Receipt of Casks of Indicated Capacities \\
\hline
\end{tabular}

21 PWR/46 BWR Assemblies

Activity

Transportation -.. Reactors to DOE Facilities ${ }^{c}$

Lag Storage ${ }^{d}$

MRS Modules

Transportation -- MRS

to Repository
(9.26 MTU)

$\$ 0.31-0.57$

79.4

35.5 (PWR)

10.1 (BWR)

None
26 PWR/52 BWR Assemblies (11.16 MTU)

$\$ 0.26-0.47$

65.9

35.5 (PWR)

17.5 (BWR)

None
32 PWR/76 BWR Assemb7ies (14.47 MTU) $\$ 0.20-0.36$ 50.8 35.5 (PWR) 19.1 (BWR)

None

a Savings are not additive, i.e., savings associated with the receipt of an individual TSC or SOC is realized in connection with only one activity.

'S Savings are expressed in $\$ / \mathrm{kgU}$ contained in the spent fuel delivered to DOE. Savings for consolidated fuel are approximately $60 \%$ of those shown in the table.

c Comparison is based on savings resulting from the use of utility-furnished TSCS in place of a DOE transport cask. However, the change in savings resulting from the use of higher capacity casks would also be the same for all of the SOC cases shown in Appendix C.

d In the case where the TSC or SOC is used to deliver spent fuel and then used for lag storage at a repository when there is also a MRS facility in the system, the avoided costs shown are reduced to about $96 \%$ of the values shown for PWR fuel and to about $72 \%$ for BWR fuel. 
The following sets forth the conclusions that can be drawn from the table along with comments regarding the impact of changes in cask cost on the results shown in the table:

(1) The savings in transport costs between the reactors and the DOE facility decline slightly with increasing capacities of the TSCS, SOCS and DOE transport casks, but are small to begin with. These savings change by about $5 \%$ for each $\$ 100$-thousand change in the cost of a cask.

(2) The savings in lag storage costs declines as the capacity of the TSC or SOC for intact fuel assemblies increases. This is because the savings to DOE are equal to the cost of the cask. When the cost is held constant, and the capacity is increased, the savings expressed as a unit of capacity declines. For each $\$ 100$-thousand change in the initial cask cost, the savings shown in the table correspondingly change by 12.5 percent of the values shown.

(3) The savings in the cost of MRS modules are independent of the capacity or cost of the TSCS or SOCs used to replace them. The greater the capacity of the cask, the more it is worth as a MRS module. However, this value has to be distributed over the larger amount of fuel that is contained in the cask as received from the utility. Thus, the savings expressed in $\$ / \mathrm{kgU}$ received in the cask does not change for increased cask capacity. Changes in the cost for the cask also do not impact savings because the savings are the result of the cost of the MRS modules, and not the casks. However, in the case of TSCS or SOCs that are received containing BWR fuel, the cost of a new basket and the cost of disposal of the old one has to be deducted from the savings that would have been experienced if the basket had not needed to be replaced. This savings increases with increasing cask capacity inasmuch as it was assumed that the cost of a new basket and disposal of the old one would not increase, and thus the deduction for these items was distributed over a larger amount of fuel received. This resulted in increasingiy smaller deductions for casks of higher capacity, and a correspondingly larger net savings.

(4) There were no savings that resulted from the use of the TSCS for transport of cans of consolidated fuel from the MRS to the repository within the range of cask capacities studied. Moreover, variations in the cost of the TSCs did not impact the transport costs since it was not included as an element of such cost. 



\section{APPENDIX J}

PROSPECTS FOR USE OF TSCS IN CONNECTION WITH THE STORAGE AND

HANDLING OF DEFENSE WASTES

TABLE OF CONTENTS

Page

1.0 USE OF TSCS FOR WEST VALLEY HIGH LEVEL WASTE J-1

1.1 Background J-1

1.2 Possible Role of TSCS in West Valley System J-3

2.0 USE OF TSCS FOR DEFENSE WASTE J-3

2.1 Background J-3

2.2 Possible Role of TSCS in Defense Waste System J-4

3.0 CONCLUSIONS REGARDING THE USE OF TSCS IN CONNECTION WITH J-4 WEST VALLEY AND DEFENSE WASTES

4.0 REFERENCES FOR APPENDIX J J $J$ 


\section{APPENDIX J \\ PROSPECTS FOR USE OF TSCS IN CONNECTION WITH THE STORAGE AND \\ HANDLING OF DEFENSE WASTES}

The purpose of this Appendix is to perform a preliminary evaluation of the feasibility of using TSCs in the storage and handling of West Valley and defense wastes.

1.0 USE OF TSCS FOR WEST VALLEY HIGH LEVEL WASTE

1.1 BACKGROUND

High level waste (HLW) from the reprocessing of spent nuclear fuel at West Valley will be solidified in canisters that are 3 meters (120") in length, and $61 \mathrm{~cm}\left(24^{\prime \prime}\right)$ outer diameter. West Valley will begin production of these canisters in late 1989. Three hundred canisters of HLW will be produced during the eighteen months vitrification campaign. The canisters will be stored on-site in a building previously used for chemical processing. The current plan is to store the HLW canisters at West valley until they can be delivered to a repository. The first delivery of HLW to a repository is scheduled for the year 2008.

Table J-1 provides the production schedule and the repository acceptance schedule for the West Valley HLW canisters (References 1 and 2). An assumption has been made that the West valley canisters will be delivered to the repository on a priority basis, meaning they will be the first canisters delivered starting in the year 2008. This assumption is based on the expressed desire of DOE to remove the canisters from the site at the earliest opportunity. Based on the above assumption and the current $\mathrm{plan}$ of storing the HLW on-site, the canisters would be in storage for a minimum of seventeen years at West Valley before shipments to a repository.

Another option being considered by DOE is the transport of the canisters to an alternate storage site prior to the time that they could be delivered to a repository. This action would allow for earlier decommissioning of the West Valley site. INEL and Savannah River are among the possible alternative storage sites. 
TABLE $\rfloor-1$

PRODUCTION SCHEQULE AND REPOSITORY ACCEPTANCE SCHEOULE FOR MLM CANISTERS

\begin{tabular}{|c|c|c|c|c|c|c|c|}
\hline \multirow[b]{2}{*}{ Year } & \multicolumn{2}{|c|}{$\begin{array}{c}\text { Canister } \\
\text { Production Schedule }\end{array}$} & \multicolumn{2}{|c|}{$\begin{array}{c}\text { Repository MLu } \\
\text { Acceptance schedule }\end{array}$} & \multicolumn{3}{|c|}{ Inventory of In-Storage Canisters } \\
\hline & Defense HLM & $\begin{array}{l}\text { West Vagley } \\
\text { HLH }\end{array}$ & $\begin{array}{c}\text { First } \\
\text { Repository }\end{array}$ & $\begin{array}{c}\text { Second } \\
\text { Repository }\end{array}$ & Defense $\mathrm{HLH}$ & $\frac{\text { Cumulative Tor }}{\text { Nest Valley }}$ & Comblned \\
\hline 1990 & $405^{d}$ & 150 & & & 405 & 150 & 555 \\
\hline 1991 & 405 & 150 & & & 810 & 300 & 1.110 \\
\hline 1992 & 405 & & & & 1.215 & 300 & 1.515 \\
\hline 1993 & 405 & & & & 1.620 & 300 & 1.920 \\
\hline 1994 & 405 & & & & 2.025 & 300 & 2.325 \\
\hline 1995 & 405 & & & & 2.430 & 300 & 2.730 \\
\hline 1996 & 405 & & & & 2.835 & 300 & 3.135 \\
\hline 1998 & 540 & & & & 3.375 & 300 & 3.675 \\
\hline 1998 & 540 & & & & 3.915 & 300 & 4.215 \\
\hline 1999 & 540 & & & & 4.955 & 300 & 4.755 \\
\hline 2000 & 540 & & & & 4.995 & 300 & 5.295 \\
\hline 2001 & 540 & & & & 5.535 & 300 & 5.835 \\
\hline 2002 & 540 & & & & 6.075 & 300 & 6.375 \\
\hline 2003 & 240 & & & & 6.315 & 300 & 6.615 \\
\hline 2004 & 240 & & & & 6.555 & 300 & 6.855 \\
\hline 2005 & 240 & & & & 6.795 & 300 & 7.095 \\
\hline 2006 & 315 & & & & 8.110 & 300 & 7.410 \\
\hline 2007 & 315 & & & & 8.840 & 300 & 7.740 \\
\hline 2008 & 515 & & $187^{c}$ & & 7.955 & 113 & 8,068 \\
\hline 2009 & 515 & & $433^{\mathrm{e}}$ & & 8.150 & 0 & 8.150 \\
\hline 2010 & 515 & & 800 & & 7.865 & 0 & 7.865 \\
\hline 2011 & 515 & & 800 & & 8.580 & 0 & 7.580 \\
\hline 2012 & 515 & & 800 & & 7.295 & 0 & 7.295 \\
\hline 2013 & 515 & & 800 & & 7.010 & 0 & 7.010 \\
\hline 2014 & 515 & & 800 & & 6.725 & 0 & 6.725 \\
\hline 2015 & 515 & & 800 & & 6.440 & 0 & 6.440 \\
\hline 2016 & 515 & & 800 & & 6.155 & 0 & 6.155 \\
\hline 2017 & 515 & & 800 & & 5.870 & 0 & 5,870 \\
\hline 2018 & 515 & & 800 & & 5.585 & 0 & 5.585 \\
\hline 2019 & 465 & & 480 & & 5.570 & 0 & 5.570 \\
\hline 2020 & 410 & & & & 5,980 & 0 & 5.980 \\
\hline 2021 & 410 & & & & 6.390 & 0 & 6,390 \\
\hline 2022 & 410 & & & & 6.800 & 0 & 6,800 \\
\hline 2023 & 410 & & & & 7,210 & 0 & 7.210 \\
\hline 2024 & 410 & & & & 7.620 & 0 & 7.820 \\
\hline 2025 & 380 & & & 800 & 7.200 & 0 & 7.200 \\
\hline 2026 & & & & 800 & 6.400 & 0 & 6.400 \\
\hline 2027 & & & & 800 & 5.600 & 0 & 5.600 \\
\hline 2028 & & & & 800 & 4.800 & 0 & 4.800 \\
\hline 2029 & & & & 800 & 4.000 & 0 & 4.000 \\
\hline 2030 & & & & 800 & 3.200 & 0 & 3.200 \\
\hline 2031 & & & & 800 & 2.400 & 0 & 2.400 \\
\hline 2032 & & & & 800 & 1.600 & 0 & 1,600 \\
\hline 2033 & & & & 800 & 800 & 0 & 800 \\
\hline 2034 & & & & 800 & 0 & 0 & 0 \\
\hline & 16.000 & $\overline{300}$ & 8.300 & $\overline{8.000}$ & & & \\
\hline
\end{tabular}

aror defense hLW there is a conversion factor of 0.5 MTU (equivalent heat)/canister

For Mest Valley HLU there is a conversion factor of 2.13 (equivalent heat)/canister

First 640 mTU HLL allocated to best valley

defense ML production schedule obtained from Tabie 7.1 of "Perspective on Methods to Calculate A Fee for Disposal of Defense Migh-Level Haste in Combined (CIvilian/Defense) Repositorles". DOE/RL-86-10

433 canisters is a sum of 113 West Valley cantsters and 320 defense HLW canisters.

fFrom the Oraft Misston PI an Amendment. January 1987 
As mentioned above, the 300 West Valley canisters will be stored in an existing building at West Valley which has been adapted for storage, and ultimately will be shipped to the repository for disposal. Storage will begin in about 1990, long before the possibility that excess TSCs would be made available from utilities or elsewhere. Thus, TSCS are not a candidate for canister storage at West Valley. However, the possibility of delays in repository startup may increase pressures for canister removal from West valley prior to repository startup. This possibility creates a potential role for TSCs in both transport and storage of canisters of West Valley waste and, depending upon the timing, such casks could be available from utility storage service. Even without early closure of the West Valiey site, TSCs could be used to transport HLW canisters from West Valley to the repository. By the year 2008, when shipments to a repository would begin, some utilities may be in a position to provide TSCS to West Valley for this purpose. However, the designs of TSCS for spent fuel storage and transport are not optimized for HLW canisters. The HLW canisters are shorter than spent fuel assemblies by about five feet, and only four canisters would fit into a TSC. Also the spent fuel assembly basket would have to be replaced with a basket sized for HLW canisters, resulting in a significant reduction in avoided costs to DOE.

At most only a few TSCS would be needed to deliver canisters to the repository at the acceptance rate of 800 canisters per year for both West Valley and defense HLW. In such cases DOE could offer a payment to utilities for savings in the transport cask cost less the added cost of a new basket, disposal of the replaced basket, and the added cost of transport resulting from the use of a less efficient cask than one specifically designed for the purpose.

\subsection{USE OF TSCS FOR DEFENSE WASTE}

2.1 BACKGROUND

HLW will be solidified in canisters at Savannah River, Hanford, and Idaho. Production for HLW canisters at Savannah River is planned to begin in 1990, at Hanford in 1997, and at Idaho in 2008. The canisters that are produced at Savannah River will be stored in vaults which consist of shielded, air-cooled buildings holding up to 1,000 canisters each (two years 
production). Delivery of the canisters to the DOE repository is scheduled to begin in the year 2008 .

Table J-1 shows both the production schedule for the defense HLW canisters as well as the projected repository acceptance schedule. The inventory of HLW canisters in storage peaks at 8,150 in the year 2009. The inventory declines from 2009 thru 2019 during which time the first repository is accepting canisters at a rate greater than the production rate. The inventory peaks again at 7,620 in the year 2024 due to a gap of five years between the last year of acceptance at the first repository and the first year of acceptance at the second repository. The gap is caused by a decision to evenly distribute the number of canisters delivered to each repository.

\subsection{POSSIBLE ROLE OF TSCS IN DEFENSE WASTE SYSTEM}

A total project cost of $\$ 105-m i 17$ ion was estimated for three storage modules (Reference 3 ). This represents a storage cost of $\$ 35,000$ per canister. DOE could offer a payment for the savings in storage cost less the cost of a new basket and the cost of disposal of the old one. The payment would be contingent on a utility's cominitment to a delivery schedule consistent with DOE needs for the storage capacity. The savings from reduced handling of the canisters that would be stored in TSCS would increase somewhat the payment DOE could offer. Payments for TSCs used to transport defense waste to the repository were discussed in Section 1.2 of this Appendix.

3.0 CONCLUSIONS REGARDING THE USE OF TSCS IN CONNECTION WITH WEST VALLEY AND DEFENSE WASTES

TSCS previously used by utilities could conceivably be used for storage and transport of HLW canisters. In the case of West Valley wastes, utility-owned TSCS would not be available in time for storage of canisters, unless those canisters were to be moved from west valley and stored elsewhere prior to disposal. However, utilities with TSCS could be in a position to deliver the casks to DOE for the transport of West Valley wastes. In the case of defense HLW, starting in 1998 some utilities may be in a position to deliver TSCS to DOE for storage of canisters as well as transport. However, a factor that reduces the attractiveness of utility-owned TSCS for storage and transport of HLW canisters in the DOE system is the non-optimuin size of the casks, i.e., about one-third of the cask capacity would not be used due to 
the shorter length of the HLW canisters compared to spent fuel assemblies. A cask optimally designed for HLW canisters would be significantly more cost effective. For example, an optimally sized 135 ton rail cask could transport up to 10 canisters, thereby reducing the number of casks (and shipments) needed to deliver canisters in TSCS at a rate that meets the repository acceptance schedule.

As additional information becomes available on the storage and transport plans for West Valley and defense HLW, avoided costs in the DOE system in connection with such wastes can be estimated more accurately and an appropriate incentive schedule developed for delivery of TSCs by utilities from at-reactor storage of spent fuel.

4.0 REFERENCES FOR APPENDIX J

(1) U. S. Department of Energy, Perspective on Methods to Calculate a Fee for Disposal of Defense High-Level Waste in Combined (Civilian/Defense) Repositories, DOE/RL-86-10, December 1986

(2) U. S. Department of Energy, Draft Mission Plan Amendment, DOE/RW-0128, January 1987

(3) U. S. Department of Energy, The Defense Waste Management PIan, DOE/DP0015, June 1983 

ORNL/Sub/86-SA094/2

ORNL/Sub/86-SA224/2

$\mathrm{HR} / \mathrm{JAT}-289$

INTERNAL DISTRIBUTION

1. C. R. Attaway

2. A. G. Croff

3-7. C. W. Forsberg

8-12. E. J. Frederick

13. I. G. Harrison

14. E. K. Johnson

15. D. S. Joy

16. S. B. Ludwig

17. I. G. Medley

18. J. R. Merriman

19. K. J. Notz

20. W. H. Pechin
21. J. E. Ratledge

22. R. R. Raw 1

23. M. J. Rennich

24. T. H. Row

25. J. H. Saling

26. L. B. Shappert

27. M. W. Wanker1

28. R. G. Wymer

29. H. R. Yook

30. Central Research Library

31. Document Reference Section

32-33. Laboratory Records

34. Laboratory Records - RC

\section{EXTERNAL DISIRIBUTION}

DOE - ORO, P. O. BOX E, Oak Ridge, TN 37831

35. Office of Assistant Manager, Energy Research and Development.

DOE - Office of Civilian Radioactive Waste Management, Forrestal Building, 1000 Independence Avenue, S.W., Washington, DC 20585

36. L. H. Barrett

37. A. B. Brownstein

38. J. H. Carlson

39. M. W. Frei

40. H. J. Hale

41. G. Hartkopf

42. S. H. Kale

43. K. A. Klein

44-47. T. D. Nguyen

48. D. E. Shelor

49. R. Stein

50. E. Svenson

51. E. Wilmot

DOE - Office of Nuclear Energy, 19901 Germantown Rd., Germantown, MD 20874

52. J. A. Tori

DOE - Chicago Operations Office, 9800 S. Cass Ave., Chicago, IL 60439

53. C. Boggs-Mayes

DOE - Idaho Operations Office, 785 DOE Place, Idaho Falls, ID 83402

54. M. J. Barainca 
DOE - Nevada Operations office, P. O. Box 14100, Las Vegas, NV 89114

55. L. Skousen

DOE - Oak Ridge Operations, P. O. Box E, Oak Ridge, TN 37831

56. P. J. Gross

57. M. M. Heiskell

58. S. S. Perkins

DOE - Richland Operations Office, P. O. Box 550, Richland, WA 99352

59. D. Kenyon

Pacific Northwest Laboratory, P. O. Box 999, Richland, WA 99352

60. G. H. Beeman

61. I. L. Clark

62. B. M. Cole

63. R. L. Engel

64. R. Heineman

65. P. N. McDuffie

66. R. W. Mckee

67. G. W. McNair

68. M. R. Shay

69. M. White

70. T. Wood

Sandia National Laboratory, P. O. Box 5800, Albuquerque, NM 87185

71. G. Allen

72. A. W. Dennis

Battelle/OSTP, 505 King Ave., Columbus, OH 43201

73. D. Dippold

74. P. Hoffman

75. W. Pardue

76. R. W. Peterson

77. K. Yates

Edison Electric Institute, 1111 19th Street NW, Washington, D.C. 20036

78. S. Kraft, UNWMG

EG\&G Idaho, Inc., P. O. Box 1625 Idaho Falls, ID 83415

79. J. Leatham

Electric Power Research Institute, P. O. Box 10412, Palo Alto, CA 94303

80. R. W. Lambert

81. R. Williams 
E. K. Johnson Associates, Inc., 10461 White Granite Dr., Suite 204, Oakton, VA 22124

82. E. R. Johnson

83. N. B. McLeod

H\&R Technical Associates, 575 Oak Ridge Turnpike, Oak Ridge, IN 37831

84-87. R. Emmett

88. W. R. Rhyne

LeBoeuf, Lamb, Leiby \& McRae, 1333 New Hampshire, NW, Suite 1100, Washington, DC 20039

89. L. Trosten, NTG

Nuclear Assurance Corp., 6251 Crooked Creek Road, Suite 200, Norcross, GA 30092

90. W. J. Lee

Science Applications Int. Corp., 101 Convention Center Dr., Las Vegas, NV 89109

91. R. Morrisette

U.S. Council For Energy Awareness, 1776 I St., NW, Suite 400, Washington, D.C. 20006

92. J.R. Siegel

Weston, 955 L'Enfant Plaza, SW., Eight Floor, Washington, D.C. 20024

93. J. DiNunno

94. W. Wowak

95-334. Given distribution as shown in DOE/OSTI under UC-71 and UC-85 (240 copies, including those for NTIS. 